University of Rhode Island

DigitalCommons@URI

Open Access Dissertations

1990

\title{
A Biosurfactant of Bacterial Origin and its Characterization
}

Mohammed I. Al-Hazmi

University of Rhode Island

Follow this and additional works at: https://digitalcommons.uri.edu/oa_diss

\section{Recommended Citation}

Al-Hazmi, Mohammed I., "A Biosurfactant of Bacterial Origin and its Characterization" (1990). Open Access Dissertations. Paper 555.

https://digitalcommons.uri.edu/oa_diss/555

This Dissertation is brought to you for free and open access by DigitalCommons@URI. It has been accepted for inclusion in Open Access Dissertations by an authorized administrator of DigitalCommons@URI. For more information, please contact digitalcommons-group@uri.edu. 
A BIOSURFACTANT OF BACTERIAL ORIGIN

AND ITS CHARACTERIZATION

BY

MOHAMMED I. AL-HAZMI

1

A DISSERTATION SUBMITIED IN PARTIAL FULFILIMEENT OF THE REQUIREMEITS FOR THE DEGREE OF

DOCTOR OF PHILOSOPHY

IN

BIOLOGICAL SCIENCES 


\section{ABSTRACT}

A microorganism capable of synthesizing biosurfactant from cheap and renewable water-soluble substrate such as carbohydrate was isolated from the environment in our laboratory. It was selected from other isolates by screening for its ability to reduce the tensiometric properties of the culture broth. The isolated organism (JIZAN-1) produced significant extracellular surfactant activity during growth in batch culture on modified mineral salts medium (MMSM) containing $0.2 \%$ glucose as a carbon source and $0.02 \%$ yeast extract as a growth cofactor. This was demonstrated by lowering of the whole broth surface tension to less than 27.0 dynes/cm and the interfacial tension to less than 2.0 dynes/cm.

The isolated organism (JIZAN-1) was identified based on morphological, cultural and biochemical tests as an Arthrobacter species. Optimum culture conditions such as the amount of glucose as the carbon source, amount of $\mathrm{NH}_{4} \mathrm{NO}_{3}$ or $\mathrm{NH}_{4} \mathrm{Cl}$ as the nitrogen source, and the environmental conditions including initial medium $\mathrm{pH}$ and incubation temperature, for growth and yield of biosurfactant were determined through batch cultivation on MMSM.

Arthrobacter JIZAN-1 was used for the development of a continuous process for biosurfactant production under carbon limitation. The goal of such a process was to produce the surfactant in a cost efficient process. 
Biosurfactant recovery procedure consisted of repeated extraction of the culture broth by chloroform at $35^{\circ} \mathrm{C}$. The chloroform extract was run in various solvent systems on silica gel TLC plates to determine the mobility of the components in the chloroform extract. The first purification step consisted of fractionating the crude chloroform extract by silica gel open column, then the surfactant \#1 which was obtained from the first step was further purified through fractionation by $\mathrm{C}_{18}$ silica (reverse phase) open column chromatography and the purified surface active compound was designated AL-Hazmi's surfactant.

After the purity of AL-Hazmi's surfactant was confirmed by TLC, followed by detection with sugar and lipid specific reagents, the structure was examined by chemical and physical methods. When AL-Hazmi's surfactant was hydrolyzed under alkaline and acidic conditions, the fatty acids released were determined by GC and the water soluble portion contents of sugars and sugar derivatives were determined by HPLC. The structure was also examined by means of ${ }^{1} \mathrm{H}$ and ${ }^{13} \mathrm{C}$ nuclear magentic resonance spectroscopy (NMR) and by infrared spectrophotometry (IR). The surfactant is characterized as a complex glycolipid consisting of a number of homologs from $C_{10}$ to $C_{20}$ saturated and unsaturated fatty acids including $\alpha$-branched- $\beta$-hydroxy fatty acids. The water soluble portion obtained after acid hydrolysis yielded raffinose, galacturonic acid and four unidentified peaks. 
ABSTRACT $\ldots \ldots \ldots \ldots \ldots \ldots \ldots \ldots \ldots \ldots \ldots \ldots \ldots \ldots \ldots \ldots \ldots$

TABLE OF CONTENTS $\ldots \ldots \ldots \ldots \ldots \ldots \ldots \ldots \ldots \ldots \ldots$ iv

LIST OF FIGURES $\ldots \ldots \ldots \ldots \ldots \ldots \ldots \ldots \ldots \ldots \ldots \ldots$ vi

LIST OF TABLES $\ldots \ldots \ldots \ldots \ldots \ldots \ldots \ldots \ldots \ldots \ldots \ldots \ldots \ldots \ldots$

ACKNOWLEDGEMENTS $\ldots \ldots \ldots \ldots \ldots \ldots \ldots \ldots \ldots \ldots \ldots \ldots \ldots \ldots$

INTRODUCTION $\ldots \ldots \ldots \ldots \ldots \ldots \ldots \ldots \ldots \ldots \ldots \ldots \ldots \ldots$

CHAPTER

I. REVIEW OF THE LITERATURE $\ldots \ldots \ldots \ldots \ldots \ldots \ldots \ldots \ldots 6$

General ........................... 6

Source of biosurfactant ............... 8

From different micobes

Bacterial surfactants

Yeast surfactants

Fungal surfactants

From different substrates

Classification of biosurfactants .......... 28

Carbohydrate--containing surfactants

Amino acids--containing surfactants

Phospholipids

Fatty acids and neutral lipids

Cell surface as biosurfactant

Biosurfactants properties ............. 44

Chemical properties

Physical properties

Production of biosurfactants ............ 48

Growth conditions for biosurfactant

production ...................... 48

Methods for biosurfactant production .... 51

Isolation and purification of biosurfactants. 58

Structure elucidation of biosurfactants..... 62

Application of biosurfactants ........... 66

II. MATERIALS AND METHODS $\ldots \ldots \ldots \ldots \ldots \ldots \ldots \ldots \ldots .69$

Organism and growth conditions .......... 69

Isolation of the microorganisms

Growth conditions

C-Sources

N-Sources

Environmental conditions

Growth phase

Identification of the microorganism 
Table of Contents (cont.)

Page

Determination of proteins, lipids and carbohydrates which excreted into the

Proteins

Lipids

Colorimetric method

Thin-layer chromotography

Gas liquid chromotgraphy

Carbohydrates

Colormetric methods

Gas-liquid chromatography

Production of biosurfactant by continuous

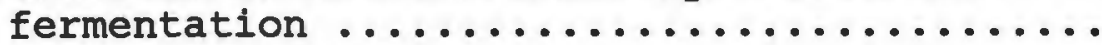

$\mathrm{NH}_{4} \mathrm{Cl}$ as $\mathrm{N}$-Source

$\mathrm{NH}_{4} \mathrm{NO}_{3}$ as $\mathrm{N}$-Source

Extraction of the biosurfactant .......... 85

Separation of chloroform extract by

thin-layer chromotography ........... 88

Purification of the biosurfactant by

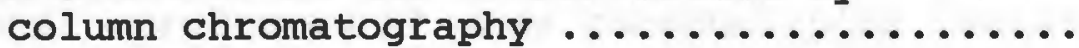

Silica gel column

$\mathrm{C}_{18}$ silica

Examination the biosurfactant structure .....

Chemical methods

Alkaline hydrolysis

Physical methods

III. RESULTS AND DISCUSSION $\ldots \ldots \ldots \ldots \ldots \ldots \ldots \ldots$

Isolation of the microorganisms ....... 94

Growth conditions from microorganisms ... 94

Identification of the microorganisms .... 141

Determination of proteins, lipids and

carbohydrates which excreted into the

culture broth during the fermentation

process ....................... 144

Biosurfactant production by continuous

fermentation ................. 177

Extraction of the biosurfactant ....... 185

Purification of the biosurfactant ...... 189

Examination of biosurfactant structure... 224

CONCLUSIONS

247

IITERATURE CITED $\ldots \ldots \ldots \ldots \ldots \ldots \ldots \ldots \ldots \ldots \ldots \ldots \ldots .249$ 


\section{LIST OF FIGURES}

Page

1. The structure of corynomycolic acid ........... 10

2. Trehalose-6-6-dicorynomycolate from

Rodococcus erythropolis ................. 30

3. Two rhamnose lipids from pseudomonas species ..... 32

4. Lactonic and acidic sophorose lipid from torulopsis mangoliae (bombicola) .................... 32

5. A generalized structure of $\alpha$-diglucosyldiglyceride.. 34

6. The structure of surfactin or subtilysin, a lipopeptide isolated from Bacillus subtilis ......

7. The structure of the ornithine-containing lipid isolated from pseudomonas rubescens ............

8. General structures of some phospholipids isolated from microorganisms

9. Isolation scheme of Arthrobacter JIZAN-1, and examiniation its ability to produce biosurfactant from water-soluble substrate (glucose) as

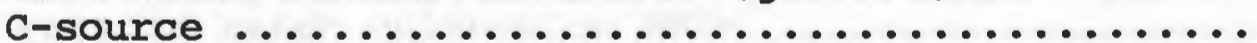

10. Extraction and purification scheme for Al-HAZMI

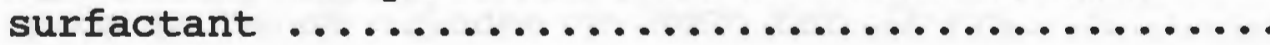

11. Structure elucidation scheme of Al-HAZMI

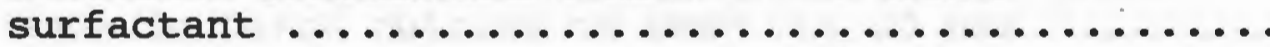

12. Surface tension profile of the whole culture broth of the isolated gram positive organisms .........

13. Effect of yeast extract or biosurfactant production by the isolated gram positive organisms .........

14. Effect of water-soluble (glucose) substrate and various water-insoluble substrates as C-sources on biosurfactant production by JIZAN-1

15. Biosurfactant production was monitored by measuring the surface and interfacial tensions by Fisher autotensiomat 
List of Figures (cont.)

$\underline{\text { Page }}$

16. a,b. Surface and interfacial tensions of the whole culture broth of JIZAN-1 as related to its growth in batch culture on BMSM containing $0.2 \%$ glucose and 0.028 yeast extract at $200 \mathrm{rpm}$ and $30^{\circ} \mathrm{C}$ for 36 hrs.

17. $a, b$. Surface and interfacial tensions of the whole culture broth of JIZAN-1 as related to its groth in batch culture on BMSM containing various amount of $\mathrm{NH}_{4} \mathrm{NO}_{3}$ as $\mathrm{N}$-source and $0.2 \%$ glucose as C-source

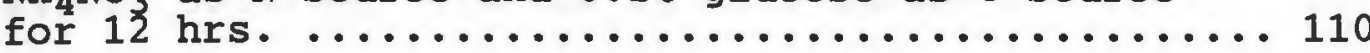

18. Arthrobacter JIZAN-1 growth rate in batch culture on MMSM containing various amount of glucose as c-source ............................

19. Effect of glucose concentration as c-source on production of biosurfactant by JIZAN-1 in batch culture on MMSM ...................... 116

20. a,b. Effect of incubation temperature on JIZAN-1 growth rate in batch culture on MMSM .......... 118

21. a. Effect of initial medium $\mathrm{pH}$ on JIZAN-1 growth rate in batch culture on MMSM .............. 121

22. Effect of $\mathrm{NH}_{4} \mathrm{NO}_{3}$ concentration on JIZAN-1 growth rate in batch culture on BMSM for $22 \mathrm{hrs}$....... 125

23. Effect of $\mathrm{NH}_{4} \mathrm{Cl}$ concentration on JIZAN-1 growth rate in batch culture on BMSM for $20 \mathrm{hrs}$....... 127

24. a,b. Effect of $\mathrm{NH}_{4} \mathrm{NO}_{3}$ concentration on biosurfactant production by JIZAN-1 batch culture on BMSM, as determined by measuring surface and interfacial tensions ....................... 130

25. Effect of $\mathrm{NH}_{4} \mathrm{Cl}$ concentration on biosurfactant production by JIZAN-1 batch culture on BMSM, as determined by measuring the surface tension .......

26. a,b. The relationship between growth (biomass concentration) of JIZAN-1 in batch culture on BMSM containing $0.15 \% \mathrm{NH}_{4} \mathrm{NO}_{3}$ and production of biosurfactant

27. a,b. The relationship between growth (biomass concentration) of JIZAN-1 in batch culture on BMSM containing $0.15 \% \mathrm{NH}_{4} \mathrm{Cl}$ and production of biosurfactant 
List of Figures (cont.)

Page

28. The reduction of surface and interfacial tensions of the whole culture broth by JIZAN-1 grown in batch culture on MMSM and at the optimum conditions obtained from the previous experiments ......... 140

29. Protein standard curve which was prepared by Folin Phenol reagent method based on beef serium albumin

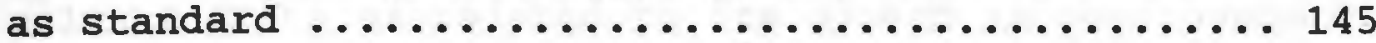

30. Gas chromatographic profile of fatty acid methyl esters obtained from GLC-10 as standard ........ 147

31. Gas chromatogram of the fatty acid methyl esters obtained from PUFA-1 as standard ............. 149

32. Gas chromatographic profile of non-volatile long chain fatty acids obtained from the culture broth after extraction by ethyl ether, saponification and derivatization

33. a,b. Gas chromatographic of the non-volatile short chain fatty acid methyl obtained from GLC-30 and

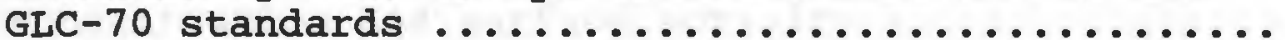

33 c. Gas chromatographic profile of the non-volatile short chain fatty acid methyl esters obtained from the culture broth after extraction, saponification and derivatization

34. a. Gas chromatogram of the volatile acids $\left(\mathrm{C}_{2}-\mathrm{C}_{7}\right)$ obtained from the standard ................. 161

34. b. Gas chromatogram profile of the volatile acids $\left(\mathrm{C}_{2}-\mathrm{C}_{7}\right)$ obtained from the culture broth

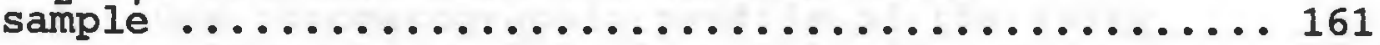

35. Glucose standard curve prepared by anthronesulfuric acid method

36. Glucose standard curve prepared by phenol-sulfuric acid method

37. Gas chromotograms obtained from xylose, ribose and rhamnose which were used as standards ....... 169

38. Gas chromotograms obtained from fructose, galactose and glucose which were used as standards ........ 172

39. a,b. Gas chromotograms obtained from glucuronic acid and galacturonic acid which were used as standards 
Iist of Figures (cont.)

Page

39. c. Gas chromotographic profile of the sugars and sugar derivatives which excreted into the culture broth during cultivation and after acid

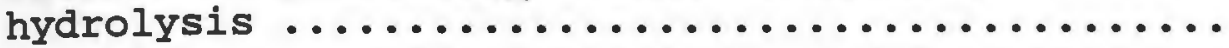

40. a,b. Surface and interfacial tensions of JIZAN-1 culture broth as related to its growth in continuous culture on BMSM containing $0.2 \%$ glucose and $0.15 \%$

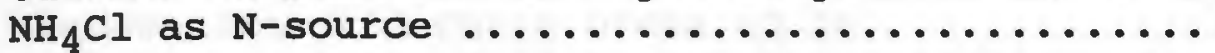

41. a,b. Surface and interfacial tensions of JIZAN-1 culture broth as related to its growth in continuous culture on BMSM containing $0.2 \%$ glucose and $0.15 \% \mathrm{NH}_{4} \mathrm{NO}_{3}$ as $\mathrm{N}$-source $\ldots \ldots \ldots \ldots \ldots \ldots$

42. Surface and interfacial tensions of the deionized water, culture broth, culture broth after extraction by chloroform at $35^{\circ} \mathrm{C}$ and chloroform

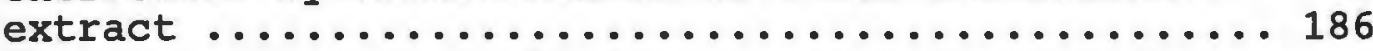

43. a,b. The correlation between surfactant \#1 concentration and surface activity ........... 192

44. Surfactant \#1 spectra, which was obtained by scanning between $\max 900-190 \mathrm{~nm}$ by UV/VIS spectrophotometer ............................ 194

45. Spectra correlation chart for the UV region ...... 195

46. $a, b$ and $c$. Gas chromatograms of the fatty acids obtained from $\mathrm{C}_{10: 0}, \mathrm{C}_{12: 0}$ and GLC-10

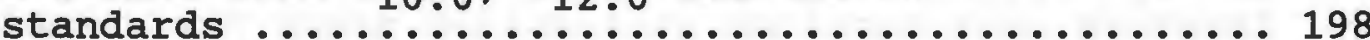

47. a,b. Gas chromatographic profile of the fatty acids obtained from GLC-20 and GLC-40 standards ... 200

48. Gas chromatogram of the fatty acids obtained from

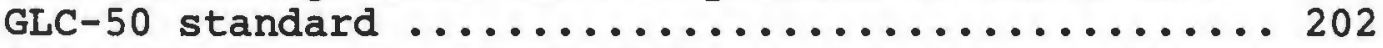

49. Gas chromotographic profile of the fatty acids

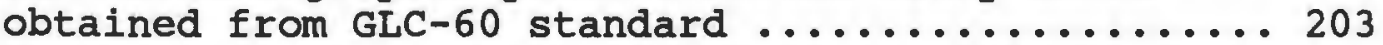

50. Gas chromatogram of the fatty acids obtained from GLC-90 standard

51. Gas chromatographic profile of the fatty acids obtained from PUFA-1 standard 
List of Figures (cont).

Page

52. Gas chromotographic profile of the fatty acids obtained from surfactant \#1 after alkaline hydrolysis, extraction and derivatization .......... 210

53. Gas chromotographic profile of the fatty acids obtained from surfactant \#1 after acid hydrolysis,

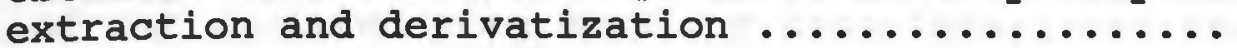

54. GLucose standard curve prepared by anthrone-surfuric acid method for surfactant \#1 sugars

55. High pressure liquid chromotographic profile of the sugars and sugar derivatives obtained from surfactant \#1 after acid hydrolysis ...........

56. The correlation between AL-HAZMI surfactant concentration aod the surface activity .......... 226

57. Gas chromotogram of the fatty acids obtained from AL-HAZMI surfactant after alkaline hydrolysis, extraction and derivatization .............. 228

58. Gas chromotographic profile of the fatty acids obtained from AL-HAZMI surfactant after acid hydrolysis, extraction and derivatization ...... 232

59. Glucose standard curve prepared by anthrone-sulfuric acid method for AL-HAZMI surfactant sugars ...... 234

60. High pressure liquid chromotographic profile of the sugars and sugar derivatives obtained from AL-HAZMI

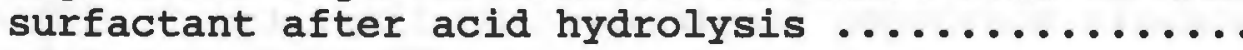

61. AL-HAZMI surfactant UV spectra, which was scanned between $\max$ 900-190 nm by UV/VIS spectrophoto-

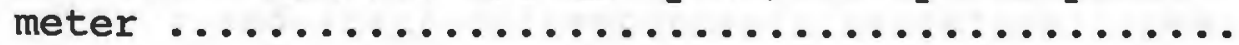

62. Infra-red spectra of AL-HAZMI surfactant in KBV

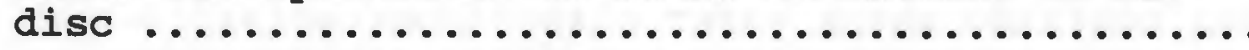

63. Proton nuclear magnetic resonance ('H NMR) spectra of AL-HAZMI surfactant

64. ${ }^{13} \mathrm{C}$-Nuclear magnetic resonance $\left({ }^{13} \mathrm{C}\right.$ NMR) spectra of AL-HAZMI surfactant 


\section{LIST OF TABLES}

Page

1. Corynomycolic acids isolated from corynobacterium lepus as determined by mass spectroscopy .........

2. Composition of the basal mineral salts medium .....

3. Average surface tension of the whole culture broth of the isolated gram positive organisms grown in batch culture on BMSM containing $0.2 \%$ glucose .....

4. Average surface tension of the whole culture broth of the isolated gram positive organisms grown in batch culture on BMSM containing $0.2 \%$ glucose and $0.02 \%$ yeast extract $\ldots \ldots \ldots \ldots \ldots \ldots \ldots \ldots \ldots \ldots$

5. Average surface tension of the culture broth of JIZAN-1 grew in batch culture on BMSM containing water-soluble (glucose) substrate and various

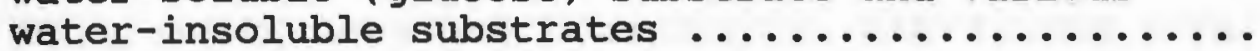

6. Fatty acids from GLC-10 standard and their correspondence retention times as determined by

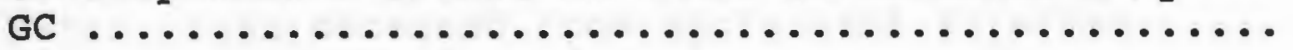

7. Fatty acids obtained from PUFA-1 standard and their correspondence retention times as determined by GC $\ldots \ldots \ldots \ldots \ldots \ldots \ldots \ldots \ldots \ldots \ldots \ldots \ldots \ldots$

8. Non-volatile long chain fatty acids obtained from JIZAN-1 culture broth and their retention times determined by GC .......................

9. Non-volatile short chain fatty acids obtained from GLC-30 and their correspondence retention times as determined by GC $\ldots \ldots \ldots \ldots \ldots \ldots \ldots \ldots \ldots \ldots \ldots \ldots \ldots$

10. Non-volatile short chain fatty acids obtained from GLC-70 and their correspondence retention times as determined by GC $\ldots \ldots \ldots \ldots \ldots \ldots \ldots \ldots \ldots \ldots$

11. Non-volatile short chain fatty acids obtained from JIZAN-1 culture broth and their correspondence retention times as determined by GC ............

12. Volatile acids $\left(\mathrm{C}_{2}-\mathrm{C}_{7}\right)$ obtained from the standard and their correspondence retention times as determind by GC 
List of Tables (cont.)

$\underline{\text { Page }}$

13. Volatile acids $\left(\mathrm{C}_{2}-\mathrm{C}_{7}\right)$ obtained from JIZAN-1

culture broth and their correspondence retention

times as determined by GC ................ 163

14. Sugars and sugar derivatives standards and their correspondence retention times as determined by GC .............................

15. Sugars and sugar derivatives obtained from JIZAN-1 culture broth and their correspondence retention

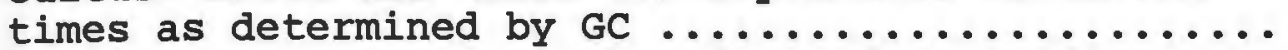

16. Fatty acids obtained from $\mathrm{C}_{10}: 0, \mathrm{C}_{12}: 0, \mathrm{GLC}-10$, GLC-20, GLC-40 and GLC-50 standards and their correspondence retention times as determined by GC $\ldots \ldots \ldots \ldots \ldots \ldots \ldots \ldots \ldots \ldots \ldots \ldots \ldots \ldots \ldots$

17. Fatty acids obtained from GLC-60, GLC-90 and PUFA-1 standards and their correspondence retention times as determined by GC $\ldots \ldots \ldots \ldots \ldots \ldots \ldots \ldots \ldots \ldots . \ldots \ldots$

18. Fatty acids obtained from surfactant \#1 after alkaline hydrolysis and their correspondence

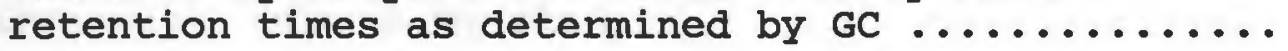

19. Fatty acids obtained from surfactant \#1 after acid hydrolysis and their correspondence retention times as determined by GC ..................

20. Sugars and sugar derivatives standards and their correspondence retention times as determined by HPLC

21. Sugars and sugar derivatives obtained from surfactant $\# 1$ after acid hydrolyis and their correspondence retention times as determined by HPCL

22. Fatty acids obtained fro AL-HAZMI surfactant after alkaline hydrolysis and their correspondence retention times as determined by GC ............

23. Fatty acids obtained from AL-HAZMI surfactant after acid hydrolysis and their correspondence retention

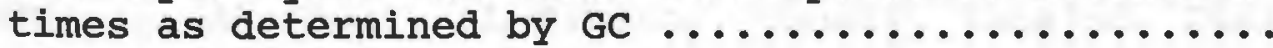

24. Sugars and sugar derivatives obtained from AL-HAZMI surfactant after acid hydrolysis and their correspondence retention times as determined by HPCL 


\section{ACKNOWLEDGEMMENTS}

All praise is due to Allah the exalted, of whom we ask help, forgiveness and guidance, and in whom we seek refuge against our own wrongdoing.

I would like to express my sincere appreciation and thanks to Dr. Traxler, who initiated the idea of this project, for his extensive help, advice and supervision that he has given me all along. I would also like to express my appreciation to Dr. M.J. Caldwell and Dr. Phyllis Brown for their help, advice and participation on my committee.

I deeply thank the officials of the Saudi Arabian Educational mission for their constant support and encouragement through my study.

To my father and mother, I can not say enough, you have always been there when I needed you and for that I owe you alot.

To my family, brothers, sisters, and relatives, I extend my sincere appreciation, gratitude, and thanks to every one of you for the continuous support, encouragement, and love.

Thanks go to all my friends especially Dr. Radain, Mr. AL-Belaikhi, AL-Yabis, Adnan Hammed, Dr. Subbagh, Mr. AL-Humizi, AL-Salamah, AL-Zawawi, AL-Rawithi, AL-Zahim, AL-Magdally, AL-Shaar, AL-Mahaini, Yousif, Tahajod, Zuhir, 
Galip, Laham, Sam, Marcia, Ms. Wood, Rifa, Mei chen, Valary and Robin for sharing many years of true friendship. Finally, my warmest appreciation and thanks to my wife for her support, patience, encouragements, and most of all being part of my life with all its happiness and sorrows. To her and my sons Ahmad, Moutasim and Abdullaziz and my daughter Ibtesam all my love and life. 


\section{INTRODUCTION}

The term "surfactant" is an abbreviation for surface-active agent, a class of chemicals uniquely active at surfaces, and which when dissolved in water tend to concentrate at the gas-liquid, liquid-liquid, or solid-liquid interfaces. Thus surfactants can be responsible for the reduction of surface tension (air-water) of aqueous media, for the reduction of interfacial tension between two immiscible phases (oil-water), and for changes in the foaming properties for aqueous mixtures. Each surfactant molecule is characterized by two functional parts: a hydrophilic (water soluble) or polar part, and a lipophilic (oil soluble) or non polar part. The surface-active properties of surfactant molecule will depend on the balance between the lipophilic and hydrophilic characteristics.

Commercially available surfactants that are chemically synthesized, seem to have certain limitations because many of them are toxic and either slowly or nonbiodegradable (Swisher, 1970). Most of them are produced from hydrocarbons which are a non-replenishable resource, and their manufacture involves a series of processing steps where several chemical reactions are followed by a number of costly purification steps. The manufacture of sodium dodecyl benzene sulfonate may actually refer to as an example of a large group of synthetic surfactants called alkyl benzene sulfonates (ABS), where corrosive chemicals 
are used and quite frequently corrosion problems are encountered in the process equipment (Garrett, 1972). Some of the limitations encountered in synthetic surfactants have stimulated research to find and produce new surfactants from microbes. Microbial surfactants (Biosurfactants) are surface active agents which are either metabolic products produce by cells or the actual cell with activity due to its surface chemistry. These biosurfactants are produced by microbial cells grown on certain hydrocarbons or on other substrates such as carbohydrates as their carbon and energy source, and they may be extracellular or they may be associated with the cell wall and are not excreted into the culture broth.

Surfactants produced by micro-organisms are usually lipids. Their properties result from a combination of apolar and non-polar moieties in a single molecule. The nonpolar or hydrophobic portion is normally a hydrocarbon. The most common example is the hydrocarbon chain of a fatty acid. The polar or hydrophilic groups include a wide range of possibilities. Examples are the ester and alcohol functional groups of neutral lipids, the phosphate containing portion of phospholipids and the sugars of glycolipids (Cooper and Zajic, 1980).

The majority of the biosurfactant-producing organisms require a hydrocarbon as the only carbon substrate for biosynthesis. Carbohydrates were rarely used as carbon and energy source for biosurfactant production. In spite of the 
temporary reduction of the price of crude oil, it is essential to isolate organisms which can assemble biosurfactants from cheap, renewable substrates if production is to be commercially feasible. The production of biosurfactants from carbohydrate substrates offers some advantages as compared with hydrocarbons. From an engineering point of view, hydrocarbon substrates require more sophisticated equipment and more power input to achieve an adequate dispersion of the insoluble hydrocarbons (Guerra-Santos et al., 1984).

Biosurfactants are important because they expand the range of available surfactant types and exhibit surface active properties differing from synthetic surfactants. Furthermore, they are usually biodegradable, which reduces the potential for pollution (Margaretis et al., 1979).

Food processing industries utilize microbes and their products extensively. These products may be specific food stabilizing agents, emulsifiers, vitamins, amino acids, proteins, food enzymes, specific fatty acids, water binding agents, flavors, and flavor enhancing agents and foaming agents. (Kosaric et al., 1987).

The requirement for surface active molecules of biological origin (biosurfactants) is increasing, owing to their potential utilization in food processing, pharmacology, and oil industries. A use of biosurfactants that showed particular promise is in the clean up of oil spills, both on water and on land (Jobson et al., 1972). An 
indirect importance of biosurfactants is that they facilitate the growth of microorganisms on hydrocarbons and a large number of useful products can be obtained from these substrates. An important product is single-cell protein (Matles et al., 1968), as well as a large number of individual amino acids. Other useful metabolites include alkane transformation products, sugar and polysaccharides, nucleic acids, vitamins and pigments (Abbott and Gledhill, $1971)$.

The aim of this investigation is to isolate a micro organism from the environment capable of synthesizing biosurfactant not from hydrocarbon substrate, but from cheap and renewable substrate such as carbohydrate, and to identify the biosurfactant, which might have applications in food processing, pharmacology, or oil industries. The possibility of discovering a new microbial gum such as xanthan, or a unique bioemusifier like emulsan, has encouraged our interest.

This investigation consist of several steps:

1. Isolation of a microorganism from the environment capable of synthesizing biosurfactant from carbohydrate substrate, by utilizing glucose as the carbon and energy source.

2. Identification of the isolated microorganism on the basis of metabolic and morphological characteristics.

3. Determination of the optimum conditions of the microorganisms growth and yield of biosurfactant. 
4. Production of a large amount of biosurfactant at the cheapest rate by using continuous fermentation.

5. Determination of carbohydrates, proteins, amino acids, and lipids, which will be excreted during the fermentation process.

6. Purification of the biosurfactant.

7. Examination of the structure of the biosurfactant by using advanced analytical techniques such as UV, IR, and NMR . 


\section{CHAPTER I \\ "REVIEW OF THE LITERATURE"}

\section{General:}

Industry uses at least $10^{6}$ tons per year of synthetic surfactants (Gerson and Zajic, 1978a). Since there is no surfactant suitable for all the potential applications, many different types of surfactants are being used, therefore it is important to develop even more to broaden the spectrum of properties available.

Microbial surfactants (biosurfactants) are surface active agents synthesized by microbial cultures, and their molecules consist of both hydrophilic and hydrophobic moieties. Characteristically these chemical compounds affect the physical characteristics of interfaces between immisicible phases.

Biosurfactants are important because they present a much broader range of surfactant types and properties than the available synthetic surfactants. Furthermore, they are usually biodegradable, which reduces the potential of pollution (Zajic et al., 1977a, b; Margaritis et al., 1979). Other advantages of microbial surfactant production are: (1) that new chemical types can be introduced which would be difficult to synthesize and (2) that these complex biochemicals can be produced relatively simply and inexpensively by microbial fermentation.

It is very important to have a method for monitoring the microbial culture for surfactant production and surface activity. The most useful method is to measure the surface 
tension of the whole broth or a dilution of the whole broth. The surface tension of pure water is 72 dyne $/ \mathrm{cm}$, at $25^{\circ} \mathrm{C}$. An effective biosurfactant can lower this to under 30 dyne/cm. If an excess of surfactant is produced, diluting the whole broth until the surface tension increases at the critical micelle concentration (CMC) gives an estimate of the amount of surfactant present (Cooper et al., 1979a). other measurements indicate surface activity, one is the lowering of interfacial tension between an aqueous phase and an immiscible organic phase, stabilization of an oil and water emulsion is a commonly used indicator. Foam formation indicates increased surface activity by increase in the surface tension of the liquid phase at the air interface.

Biosurfactants are of increasing interest for commercial use because of the continually growing spectrum of available substances. They are biodegradable, often nontoxic, and can be produced by microbial processes, in which simple substrates such as n-alkanes, vegetable oils, and carbohydrates or even industrial waste products can serve as substrates (Kosaric et al., 1984).

While many biosurfactants, show good physiochemical properties (e.g., low critical micelle concentrations in the range of a few $\mathrm{mg} / \mathrm{L}$, reduction of interfacial tension between water and hydrocarbons to less than 1 dyne/cm, and temperature stability in a wide temperature range), their production costs are often the limiting factor which prevents their commercial use. Therefore, microbial 
surfactants (Biosurfactants) must compete with surfactants from petrochemical origin in three respects, cost, functionality, and production capacity, in order to meet the needs of the intended applications (Kosaric et al., 1983).

II. Sources of Biosurfactants:

A. From different microbes:

Biosurfactants are synthesized by bacteria, yeasts, and fungi during cultivation on various carbon sources, in particular during growth on hydrophobic substrates such as hydrocarbons.

\section{Bacterial surfactants:}

The great number of studies on n-alkane utilizing bacteria from Zajic and coworkers should be noted. These publications represent an excellent survey on biosurfactants. Gerson and Zajic in 1977 isolated a Corynebacterium lepus from natural asphalt deposits, which is distinguished by the production of large quantities of surface active material when grown on hydrocarbons. The surfactant is produced over a relatively short period of time in the exponential phase of growth, and it then degrades. During growth, the surface tension of the medium quickly fell from 72 dyne/cm to under 30 dyne/cm, and the surface active agent was produced in quantities greatly in excess of the critical micelle concentration. With the best samples it was necessary to dilute portion of $\underline{c}$. lepus culture medium 200 to 500 times before the surface tension rose above 30 dyne/cm. The peak of maximum surfactant 
concentration occurs at the time of the relative maximum oxygen consumption rate. They also noticed that the biosurfactant produced by $\underline{c}$. lepus produces a fine emulsion of kerosene in water.

Analysis of the surfactants produced by $\underline{\mathrm{c}}$. lepus grown on kerosene as the sole carbon source, showed that they were lipids containing a mixture of corynomycolic acids as shown in Fig. 1 (early appearing surfactant) and lipopeptide containing corynomycolic acids plus small amounts of several phospholipids and neutral lipids (later appearing surfactant's) in fermentation, were identified by thin-layer chromatography (Cooper et al., 1979).

Corynebacterium lepus also produced a significant amount of cell bound surfactant when grown in a mineral salts medium containing glucose as the sole carbon source, and a considerable amount of surfactant was released from the cells when some alkenes or a mixture of alkenes were added to the water suspension of the cells (Duvnjak and Kosaric, 1981). Both surface and interfacial tensions of the suspension were reduced to $30-32 \mathrm{mN} / \mathrm{m}$ and $1-2 \mathrm{mN} / \mathrm{m}$ respectively as a consequence of this release. Duvnjak and Kosaric, 1985 also noticed that, alkanes with different number of carbon atoms released the surfactant from Corynebacterium lepus cells grown on mineral salts medium, containing glucose as the sole carbon source at different rates and amounts. They found that, when hexadecane was added to the cell suspension, surface and interfacial 


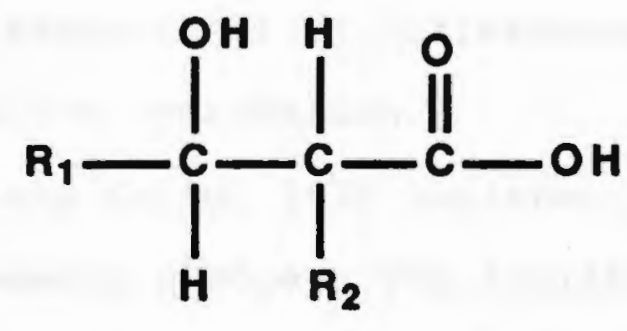

Fig.1. The structure of corynomycolic acid : a $\beta$-hydroxy $\alpha$-branched carboxylic acid in which $R_{1}$ and $R_{2}$ are alkyl substituents having a total of fewer than 40 carbon atoms. the related mycolic acids have longer substituents ( up to 90 carbon atoms, and may carry various functional groups. 
tensions were rapidly reduced to their final value, and the release of the surfactant with tetradecane was slower than with hexadecane, but the final amount of surfactant released was comparable. The rate and the amount of surfactant released by octane and decane was much lower. This phenomenon may be either due to different rates of hydrocarbon transport or to differences in solubility of the surfactant in the hydrocarbon.

Panchal and Zajic, 1978 isolated Corynebacterium sp. PPS-11 from sewage sludge. The isolated microorganism was monitored 'for growth on minimal medium contaning $\mathrm{n}$-hexadecane as the carbon and energy source. They found that during fermentation surface active agents were released into the medium, the surface active properties were associated with the lipid fractions, since most of the emulsifying activity was located in the chloroform-methanol extract. The bioemulsifiers produces by Corynebacterium sp. PPS-11 grown on mineral salts medium supplemented with aliphatic hydrocarbons were isolated by Panchal et al. (1978). The lipid extract had hydrophobic properties and the polysaccharide polymer was hydrophilic. These emulsifiers were used to prepare multiple-phase w/o/w emulsions, and the percentage formation of the multiple emulsion was found to be a function of the concentration of the two emulsifiers, and they noticed that very low concentration of the lipid extract (about $0.10 \% \mathrm{~W} / \mathrm{V}$ ) gave excellent percentage formation of the multiple emulsion 
(about 90\%), and the emulsion stability ranged from a few hours to several days.

several types of surface active compounds produced by corynebacterium fascions CFIS were isolated from culture media containing various carbon substrates including both alkanes and Carbohydrates (Cooper et al., 1982). The lowering of surface and interfacial tensions was mainly due to a mixture of extracellular neutral lipids. Emulsion stabilization and de-emulsification properties were primarily associated with the bacterial cells. They also noticed that $\underline{c}$. fascions produces much larger amounts of extracellular neutral lipids when alkane was used as the substrate, but the ability to emulsify kerosene and water mixtures was comparable for the cells grown with carbohydrates or alkanes. The surprising result they found

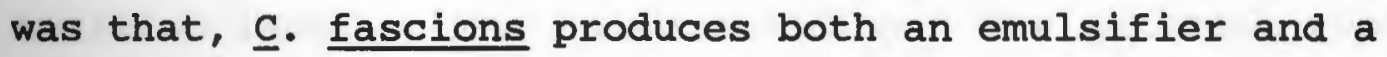
de-emulsifier. Corynebacterium hydrocarboclastus produced an extracellular lipopolymer with emulsifying properties when grown on a mixture of linear hydrocarbons (zajic et al., 1977). This microorganism was found to grow well on a variety of carbohydrates and hydrocarbons. However the best substrates were found to be a pure linear hydrocarbons and particularly $\mathrm{n}-\mathrm{C}-{ }_{12}, \mathrm{n}-\mathrm{C}_{13}$, and $\mathrm{n}-\mathrm{C}_{14}$, and the substrates supporting good growth gave good polymer production.

Continuous fermentation technique was applied for production of the extracellular polymer by growing Corynebacterium hydrocarboclastus on kerosene as the carbon 
and energy sources in a 24 liter reactor (Zajic et al., 1977). They found that this polymer was composed of protein, lipid, and carbohydrates. This polymer possessed surface active properties and had two critical micelle concentrations. Two microorganisms isolated from soil, were able to grow on the polymer as a sole carbon and energy source, thus proving its biodegradability. These microorganisms were tentatively identified as Flavobacterium brevi and Flavobacterium devorans.

Growth of Arthrobacter paraffineus KY4303, like many other hydrocarbon-degrading microorganisms, is accompanied by emulsification of the hydrocarbon substrate in the growth medium. Suzuki et al (1969) reported that, Arthrobacter paraffineus KY4303 when grown on n-paraffin as the sole source of carbon, produced anthrone-positive lipid in the emulsion layer of the culture medium. The anthrone-positive lipid was isolated and identified as $\alpha$-branched- $\beta$-hydroxy fatty acid trehalose ester. The addition of penicillin to the growing culture caused a significant suppression of trehalose lipid formation and led consequently to the accumulation of both precursors, $\alpha-\alpha$-trehalose, and $\alpha$-branched- $\beta$-hydroxy fatty acid in the culture medium (Suzuki et al., 1969). A small amount of cupric ion contaminating the culture medium of Arthrobacter paraffineus KY4303 was found to cause an increase in the production rate of L-glutamic acid and $\alpha$ - $\alpha$-trehalose (Suzuki et al., 1971). Suzuki et al. (1974) reported that the n-alkane-utilizing 
bacteria (Arthrobacter paraffineus KY 4303) which is capable of producing trehalose lipid from n-alkane, synthesized two kinds of sucrose lipids when grown on sucrose in place of n-alkane as the sole carbon source. They found that the major components of these lipids were sucrose and $\alpha$-branched- $\beta$-hydroxy fatty acid. Formation of sucrose lipids was also demonstrated in sucrose grown cells of several stains of Corynebacterium, Nocardia, and Brevibacterium, which were isolated as hydrocarbon-utilizing bacteria and could produce a considerable amount of trehalose lipid from n-alkane. When Arthrobacter paraffineus KY 4303 was grown on fructose as the sole carbon source, it produced two novel glycolipids, both of which were different from trehalose-lipid produced from n-alkane and sucrose-lipids from sucrose by the same microorganisms (Itoh and Suzuki, 1974). Two kinds of glycolipids were isolated, one of the glycolipids was identified as fructose-6-corynomycolate, and the other was identified as fructose 1,6-dicarynomycolate. Formation of the fructose lipids was also demonstrated in fructose grown cells of several strains of Corynebacterium, Nocardia, Mycobacterium and Brevibacterium.

Arthrobacter paraffineus ATCC 19558 grown in MMSM (modified mineral salts medium) containing hydrocarbon, produced surfactant with maximum CMC value obtained by using hexadecane as the carbon source (Duvnjak et al., 1982). They observed no surface active agent in whole broth 
when glucose was used in MMSM medium instead of hexadecane. In the process of surfactant production, glucose can be used as a carbon source for growth, and hexadecane added later can serve for production of the surface active agent. Resting cells of Arthrobacter sp. DSM2567 incubated in the presence of various mono-, di-, or trisaccharides, biosynthesized different glycolipids (LI et al., 1984). All eight glycolipids contained the corresponding carbohydrates moiety and one, two, or three $\alpha$-branched- $\beta$-hydroxy fatty acids were produced when mannose, glucose, cellobiose, maltose, or maltotriose were used as carbon sources in a simple phosphate buffer. It has been known that rhamnolipid was produced by cultivation of Pseudomonas aeruginosa on

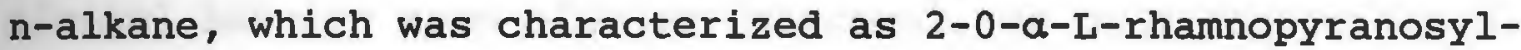
$\alpha-L-r h a m n o p y r a n o s y l-\beta-h y d r o x y d e c a n o y l-\beta-$ hydroxy-decanoate (Burger et al., 1963). The study of the biosynthesis of this lipid suggested the presence of another rhamnolipid as a precursor of the former. In the course of screening studies, Itoh et al. (1971) found that Pseudomonas aeruginosa grown on n-paraffin as the sole source of carbon produced considerable amounts of mycoplasmacidal substances, these were isolated and characterized as rhamnolipids (R-1 and R-2). R-1 was the known rhamnolipid, while R-2 was the newly isolated compound which was postulated as the precursor of the former, and it was identified as $\alpha$-L-rhamnosyl- $\beta$-hydroxy decanoyl- $\beta$-hydroxydecanoate. 
Hisatsuka et al. 1971, reported the formation of rhamnolipid by a hydrocarbon-utilizing bacterium, Pseudomonas aeruginosa $\mathbf{S}_{7} \mathrm{~B}_{1}$, and it's growth stimulating effect only on n-hexadecane medium. The anthrone positive substance was found to be the most effective on the growth of this strain. During the study of n-alkane oxidation by Pseudomonas aeruginosa $S_{7} B_{1}$ by (Hisatsuka et al., 1972), the existence of a protein-like activator for n-alkane oxidation besides rhamnolipid was found in the culture broth. The purified activator, which was positive for protein, stimulated the oxidation ' of $n$-hexadecane. The protein-like activator (PA) for $n$-alkane oxidation was formed by Pseudomonas aeruginosa $\mathrm{s}_{7} \mathrm{~B}_{1}$ from long-chain $\mathrm{n}$-alkanes, 1-hexadecane, and acetyl alcohol but not from glucose, glycerol, or palmitic acid (Hisotsuka et al., 1977).

Pseudomonas aeruginosa DSM 2659 was isolated from soil samples and used for the development of a continuous process for biosurfactant production by cultivation on glucose containing medium (Guerra-Santos et al., 1984). They identified the surface active compounds as rhamnolipids, and they noticed that the biosurfactant production was poor in the presence of yeast extract.

Pseudomonas PG-1 cultivated on pristane produced high yield of a heat-stable polymeric substance which showed strong hydrocarbon emulsifying and solubilizing properties (Reddy et al 1983). The substance was isolated in crude 
form and was found to contain $34 \%$ protein, $16 \%$ carbohydrate, and $40 \%$ lipid.

An ornithine-containing lipid was found in cells of Pseudomonas rubesceus grown on nutrient agar, but not in those grown in nutrient broth (Wilkinson, 1972). The lipid is derived from L-ornithine which contains both hydroxy and non-hydroxy fatty acids.

Bacillus subtilis produces a lipopeptide, called surfactin, with exceptional surface activity (Arima, et al., 1968). This compound (surfactin) inhibits fibrin clot formation' and also lowers the surface tension of water from $72 \mathrm{mN} / \mathrm{m}$ to $27 \mathrm{mN} / \mathrm{m}$. This is significantly lower than any biosurfactant surface tension data reported in the literature (Cooper and Zajic, 1980). Normally, even the most effective biosurfactant do not reduce the surface tention of water below $30 \mathrm{mN} / \mathrm{m}$. This compound (surfactin) has been characterized as a cyclic lipopeptide which consists of L-ASP-L-Glu, L-Val, L-Leu, D-Leu and a fatty acid in the molecular ratio of 1:1:1:2:2:1 (Kakinuma et al., 1969), and the fatty acid was briefly mentioned to be $C_{15}$ isohydroxy acid (Kakinuma et al., 1969). A good yield of surfactin was obtained from glucose substrate fermentation by continuously removing the product by foam fractionation (Cooper et al., 1981). They also found that the surfactin could be easily recovered from the collapsed foam by acid precipitation, and the yield was also improved by the addition of either iron or manganese salts. Hydrocarbon addition to the medium, which 
normally increases biosurfactant production, completely inhibited surfactin production by Bacillus subtilis.

A biosurfactant-producing Bacillus licheniformis JF-2 was isolated from oil field injection water (Jenneman et al., 1983). This Bacillus sp. produced a biosurfactant when grown at $40^{\circ} \mathrm{C}$ in a sucrose mineral salts medium containing $5 \%$ Nacl. The biosurfactant produced by JF-2 appears to be quite similar to surfactin, a lipopeptide surfactant produced by Bacillus subtilus. Both compounds are ionic, are soluble in dichloromethane, contain a free amine group and a lipid moiety, and have similar surface tension-lowering abilities and critical micelle concentrations. B. Lecheniformis JF-2 and the surfactant that it produces have many properties that are desirable for enhanced oil recovery operations. Strain JF-2 grows in medium with $\mathrm{NaCl}$ concentrations up to $10 \%$ over a wide range of temperature (up to $50^{\circ} \mathrm{C}$ ) and $\mathrm{pH}$ values $(4.6$ to 9.0) and is not inhibited by the presence of crude oil (Jenneman et al., 1983) as is B. subtilis (Cooper et al., 1981). Bacillus licheniformis JF-2 anaerobically produced biosurfactant when grown on glucose-mineral salts medium containing yeast extract and $\mathrm{NaNO}_{3}$ (Javaheri et al., 1985). The surface tension of the medium was reduced from $74 \mathrm{mN} / \mathrm{m}$ to as low as $28 \mathrm{mN} / \mathrm{m}$ due to the production of an anionic biosurfactant.

In 1987 Cooper and Goldenberg studied the production of bioemulsifers by two Bacillus species. They were distinctly 
different compounds. They found that, the Bacillus sp. strain IAF-343 produced usually high yields of extracellular biosurfactant when grown on a medium containing only water soluble substrates. The neutral lipid product had significant emulsifying properties. On the same medium, Bacillus cereus IAF-346 produced a more conventional polysaccharide bioemulsifier, but it also produced a monoglyceride biosurfactant. The bioemulsifier was found to contain substantial amounts of glucosamine, and the monoglyceride lowered the surface tension of water to 28 $\mathrm{mN} / \mathrm{m}$.

Mycobacterium tuberculosis, strain $\mathrm{H}_{37} \mathrm{RV}$ was reported to produce an unusual sulfated glycolipid, which was characterized as a 2,3,6,6-tetracyl-trehalose 2-sulfate (Goren, $1970 \mathrm{a}, \mathrm{b}$ ). Myocobacterium phlei synthesizes an homologous series of polyunsaturated fatty acids, these acids will be refered to as "phleic acids". A series of esters of trehalose and phleic acids, called "lipids P" have been isolated from Mycobacterium phlei (Asselineau et al., 1972). "Lipid P" appear to be the main form of phleic acid derivatives. They are located in the outer part of the cell, and can be extracted by a simple washing of the bacteria with hexane; bacterial cells are still living after this treatment. Cells of the paraffin oxidizing bacterium Mycobacterium paraffinicum were found to contain at least five trehalose lipids (Batrakov et al., 1981). 
Nocardia erythropolis grown in aerobic submerged culture conditions on hexadecane produces a biosurfactant which reduced the surface tension of the broth (Margaritis et al., 1979). The best medium composition was found to be a simple mineral salts solution with $2 \%$ hexadecane and $0.02 \%$ yeast extract. The kinetics of both biomass and biosurfactant production was found to depend appreciably on the agitation rate. In addition, it was observed that the average size of the cells was decreased as the agitation rate was increased. Approximately $90 \%$ of the surface-active materials produced by N. erythropolis are pentane-extractable neutral lipids (Macdonald et al., 1981). The reminder of the activity is due to polar lipids. The carboxylic acids are a major component of the biosurfactant mixture, and they reflect the nature of the alkane substrate.

Rhodococcus erythropolis was found to produce certain lipids with surfactant properties when grown on n-alkanes (Kretschmer et al., 1982). The major components were trehalose lipids which were identified as a-a-trehalose-6-6-dicorynomycolate (Rapp et al., 1979), and a-a-trehalose-6-monocorynomycolates (Kretschmer et al., 1982). Rhodococcus erythropolis was cultivated in shake flasks on a mixture of $\mathrm{C}_{14}-\mathrm{C}_{15}$ n-alkanes or kerosene under growth limiting conditions such as $\mathrm{N}$-limitation or limitation of the multivalent cations. For example, nitrogen deficiency caused the formation of new glycolipids in fermentation broth 
(Ristau and Wagner, 1983). These lipids were identified as

trehalose-2,3,4,2-tetraesters.

Gluconobacter cerinus produced an ornithine and taurine containing lipid named Cerilipin (Tahara et al., 1976). Iipids with lysine are reported by Tahara et al. 1976, using Agrobacterium tumefaciens.

ornithine-containing lipid was described by Knoche and Shirely, 1972, using Thiobacillus thiooxydans. Data obtained from this investigation indicated that the $\alpha$-amino and the carboxyl group of ornithine were free.

Clostridium pasteurianum grown anaerobically on sucrose was reported to produce surface active compounds (Cooper et al., 1980). A surface active mixture of at least four neutral lipids was isolated from the culture supernatant by foam fractionation, one of these lipids appears to be a triglyceride and two of the others may be esters.

Pigmented and non-pigmented strains of Serratia marcescens produced extracellular vesicles that had wetting activity when grown at $30^{\circ} \mathrm{C}$ (Matsuyama et al., 1986). Three lipids having the wetting activity $w_{1} \cdot w_{2}$ and $w_{3}$, were isolated by thin-layer chromatography. The isolated wetting agents showed the ability to lower surface tension.

The hydrocarbon degrading microorganism Acinetobacter RAG-1 produced an extracellular non-dialyzable emulsifying agent (termed emulsan). Emulsan was prepared by heptane extraction or precipitation between 30 and $40 \%$ ammonium 
sulfate saturation (Rosenberg et al., 1979a). For the partically purified emulsifier (Emulsan) to induce wdrocarbon in water emulsions efficiently, the hydrocarbon substrate must contain both aliphatic and cyclic components (Rosenberg et al., 1979b). Extracellular emulsifier (Emulsan) was deproteinized by hot phenol treatment and purified by fractional precipitation with ammonium sulfate (zukerberg et al., 1979). They found that emulsan is highly acidic polysaccharide, consisting of a polysaccharide backbone composed of $\mathrm{N}$-acetyl galactosamine and $\mathrm{N}$-acetyl galactosamine uronic acid esterified with fatty acids, with an average molecular weight close to $10^{6}$. Mild alkaline hydrolysis released fatty acids with an average molecular weight 231, and strong acid hydrolysis yielded D-glucose (minor), D-galactosamine (major) and galactosamine uronic acid (major).

A mixed bacterial population was obtained by enrichment culture that was capable of degrading emulsan and using it as a carbon source (Shoham et al., 1983). From this mixed culture, an emulsan-degrading bacterum, termed YUV-1, was isolated. Strain YUV-1 is an aerobic, gram-negative, non-spore forming, rod-shaped bacterum which grows best in media containing yeast extract.

Acinetobacter calcoaceticus strains A2 isolated by enrichment culture techniques, were shown to produce extracellular, non-dialyzable materials which disperse 
limestone powders in water (Rosenberg et al., 1988). These materials are referred to as biodispersan. An optimized medium for the production of biodispersan was developed with ethanol as the substrate., The biodispersan was concentrated by ammonium sulfate precipitation and deprotenized by hot phenol treatment (Rosenberg et al., 1988). The active component was an anionic polysaccharide with average molecular weight $51,400 .{ }^{13} \mathrm{C}-\mathrm{NMR}$ indicated the presence of four different monosacchrides. Strong acid hydrolysis of the biodisperson yielded four reducing sugars: glucosamine, 6-methyl-áminohexose, galactosamine uronic acid, and unidentified amino sugar.

\section{Yeast surfactants:}

Candida lipolytica produces an inducible extracellular emulsifier when grown on a number of water immiscible carbon substrates (Cirigliano and Carman, 1984). This emulsifier (liposan) was partially purified, and found to form stable oil in water emulsions with a number of water-immiscible compounds. The water soluble bioemulsifier (liposan) was purified from the yeast, it consists of approximately $83 \%$ carbohydrates and $17 \%$ protein (Cirigliano and Carman, 1985).

Candida petrophilum produced emulsifying factors (protein-containing surfactant) during hydrocarbon assimilation (Iguchi et al, 1969).

Candida bogariensis was reported to produce a sophorose-lipid when grown on glucose containing medium (Cutlex and Light., 1979). 
Torulopsis bombicola was selected for its ability to produce higher yields of biosurfactant than other biosurfactant-producing microbes. In suspended cell batch cultures of $\underline{T}$. bombicola, final product concentration of 67.0 $\mathrm{gm} / \mathrm{L}$ of biosurfactant have been reported by (Cooper and Paddock, 1984). A strain of $\underline{T}$. bombicola has been isolated in Japan which can produce up to $120-130 \mathrm{gm} / \mathrm{L}$ of biosurfactant (Inoue and Ito, 1982).

Cooper and Paddock, 1983, reported that Torulopis petrophilum can synthesize either glycolipid surfactant or protein emulsifier depending on the substrate used.

Glycolipid surfactant was produced when glucose used as substrate, and protein emulsifier was produced when hydrocarbon used as substrate.

\section{Fungal surfactants:}

Ustilagic acid and a mannosyl erythritol lipid are known fungal surfactants.

Ustilago zeae was found to produce ustilagic acid which was identified later as a cellobioselipid when the corn smut fungus was grown on glucose cultures (Haskins, 1950). Another Ustilago sp. PRL-627 was reported to produce another glycolipid (erythritol-mannose lipid) when grown on glucose as the carbon source (Boothroyed et al., 1956). Also erythritol-and mannose containing glycolipids have been found intracellularly in Ustilago maydis (Fluhartz and Obrien, 1969). The fatty acid constituents were found to range from $\mathrm{c}_{12}-\mathrm{c}_{18}$ with $\mathrm{c}_{16}$ acids predominating. 
The smut fungus Shizonella melanogramna produced a new annose-and erythrose-containing lipids shizonellin $A$ and $B$ (Deml et al., 1980).

Kawashima et al. (1983), reported the production of oxtracellular mannosyl erythritol lipid by a mutant of Candida sp. $\mathrm{B}_{7}$ from n-alkanes and triacylglycerols. The fatty acid moiety was found to be a mixture of $\mathrm{C}_{7}-\mathrm{C}_{17}$ fatty acids.

\section{B. From different substrates:}

Many microorganisms are able to grow on hydrocarbons as the only carbon and energy source. A hydrocarbon by definition contains only two elements, carbon and hydrogen. Hydrocarbons encompass a wide variety of compounds including alkanes, alkenes, alkynes, cyclic, aromatic, and polyaromatic hydrocarbons. While yeasts and fungi utilize preferably unbranched, saturated n-alkanes, some bacteria degrade in addition isoalkanes and cycloalkanes as well as unsaturated and aromatic compounds (Kosaric et al. 1987).

The hydrocarbon-degrading enzymes are integrated in the cytoplasmic membrane, so that the hydrocarbon substrate has to be transported through the outer hydrophilic cell wall into the cell. This step of the hydrocarbon uptake is strongly limited by the hydrophobic character of these compounds. The microorganisms solve this problem differently. Some bacteria and yeasts excrete ionic surfactants, which emulsify the hydrocarbon substrate in the growth medium. Examples for this group of biosurfactants 
are the rhamnolipids, produced by different Pseudomonas species (Reiling et al., 1986), or the sophorolipids produced by several Torulopsis species (Inone and Ito, 1982). On the other side there are microorganisms capable of changing the structure of their cell wall. They achieve this by building Mpopolysaccharides or nonionic surfactants into their cell wall structure. To this group of micro- organisms belong Candida lipolytica and Candida tropicalis, which produce cell-wall bound lipopolysaccharides when growing on $\mathrm{n}$-alkanes (Osumi et al., 1975; Fukui and Tanaka; 1981), and Rhodococcus erythropolis and several Mycobacterium and Arthrobacter species, which synthesize nonionic trehalose corynomycolates (Rapp et al., 1979; Batrakov et al., 1981; Suzuki et al., 1969). In all cases the microorganisms achieve the same effect; they facilitate the passive diffusion of the hydrocarbon substrate into the cell. This is an explanation for the wide spectrum of different substances produced by microorganisms, which are called "biosurfactant".

The majority of the biosurfactant-producing organisms require a hydrocarbon as a carbon substrate for biosynthesis. It has been postulated that the biological function of surface active compounds is related to hydrocarbon uptake and therefore a spontaneous release occurs with these substrates (Hisatsuka et al., 1977; Holdom and Turner., 1969; Kappeli and Finnerty., 1980; Rapp et al., 1979). 
The main problem in the hydrocarbon degradation by microorganisms is the water insolubility of these substrates, therefore, hydrocarbon substrates require more sophisticated equipment and more power input to achieve an adequate dispersion of the insoluble hydrocarbons. In addition, the availability of hydrocarbons is limited if application of biosurfactants other than in enhanced oil recovery are envisaged (Guerra-Santos et al., 1984).

Carbohydrates were rarely used as carbon and energy source for biosurfactants production with a few exceptions, one of the exception is shown by Bacillus subtilus, which as has been shown to grow on blood agar plates and produce its biosurfactant "surfactin" (Mulligan et al., 1984). An interesting observation was made by Duvnjak and Kosaric in (1981) using Corynebacterium lepus for biosurfactant production in hydrocarbon and carbohydrate media. The bacterium was capable of producing a large amount of biosurfactant in both hydrocarbon and glucose media, however, the biosurfactant in the glucose media was cell-bound and could be subsequently extracted with organic solvents, whereas, the hydrocarbon produced surfactant is secreted. Another bacterium that does not require a hydrocarbon substrate but can be grown on glucose and mannose is Arthrobacter sp. DSM2767 ( $\mathrm{Li}$ et al., 1984). This opens some new possibilities in utilizing waste carbohydrate and cheap carbohydrate media for biosurfactant production. 
III. Classification of biosurfactants:

Biosurfactants are molecules which including both lipophilic and hydrophilic moieties-the common lipophilic moiety is the hydrocarbon chain of a fatty acid, and the hydrophilic group is derived from the ester or alcohol Functional groups of neutral lipids, the carboxylate group of fatty acids or amino acids, the phosphate-containing portions of phospholipids, and the carbohydrates of glycolipids.

Basically, there are five major classes of

\section{blosurfactants :}

A. Carbohydrate-containing surfactants.

B. Amino acid-containing surfactants.

C. Phospholipids.

D. Fatty acids and neutral lipids.

E. Cell surface as a biosurfactant.

These five classes of biosurfactants are produced under different conditions and in different systems have the ability to lower the surface tension and interfacial tension of oil-water mixtures.

\section{A. Carbohydrate-containing surfactants:}

The most commonly isolated microbial surfactants, or emulsifying agents are glycolipids. Extracellular biosurfactants produced by microorganisms are usually glycolipids.

\section{Trehalose lipid:}

Glycolipids containing the dissaccharide trehalose are 
common in the literature of the extracellular lipids of coryneform and related bacteria.

Trehalose containing glycolipids have been isolated from several strains of Arthrobacter, Mycobacterium, Brevibacterium, Corynebacterium and Nocardia growing on hydrocarbons (Suzuki et al., 1969; Rapp and Wagner, 1976).

Trehalose lipid was isolated from Arthrobacter paraffineus KY4303 grown on n-paraffins (Suzuki et al., 1969). This glycolipid showed strong emulsifying properties, and each molecule contain trehalose and two $\alpha$-branched- $\beta$-hydroxy fatty acids (Fig. 2 ).

The most extensively studied trehalose lipids are the cord factor, originally isolated from Mycobacteria but eventually found in a wide range of related organisms (Lederer, 1967; Asselinean, 1966).

Ristau and Wagner, 1983 reported that the Arthrobacter sp. strain DSM 2567 produce surface and interfacial active compounds when grown on a mixture of $\mathrm{C}_{14}-\mathrm{C}_{15} \mathrm{n}$-alkanes. A new ionic $\alpha$-a-trehalose-tetraester was the main component. Most of the trehalose lipids characterized have had the same general structure of two $\alpha$-branched- $\beta$-hydroxy fatty acids esterified to a trehalose molecule.

\section{Rhamno lipids:}

Glycolipids that contain the sugar rhammose and $\beta$-hydroxy carboxylic acids have been isolated from several strains of Psueodomonas aeruginosa. The first rhamnolipid to be characterized (R-1) consisted of two molecules of 


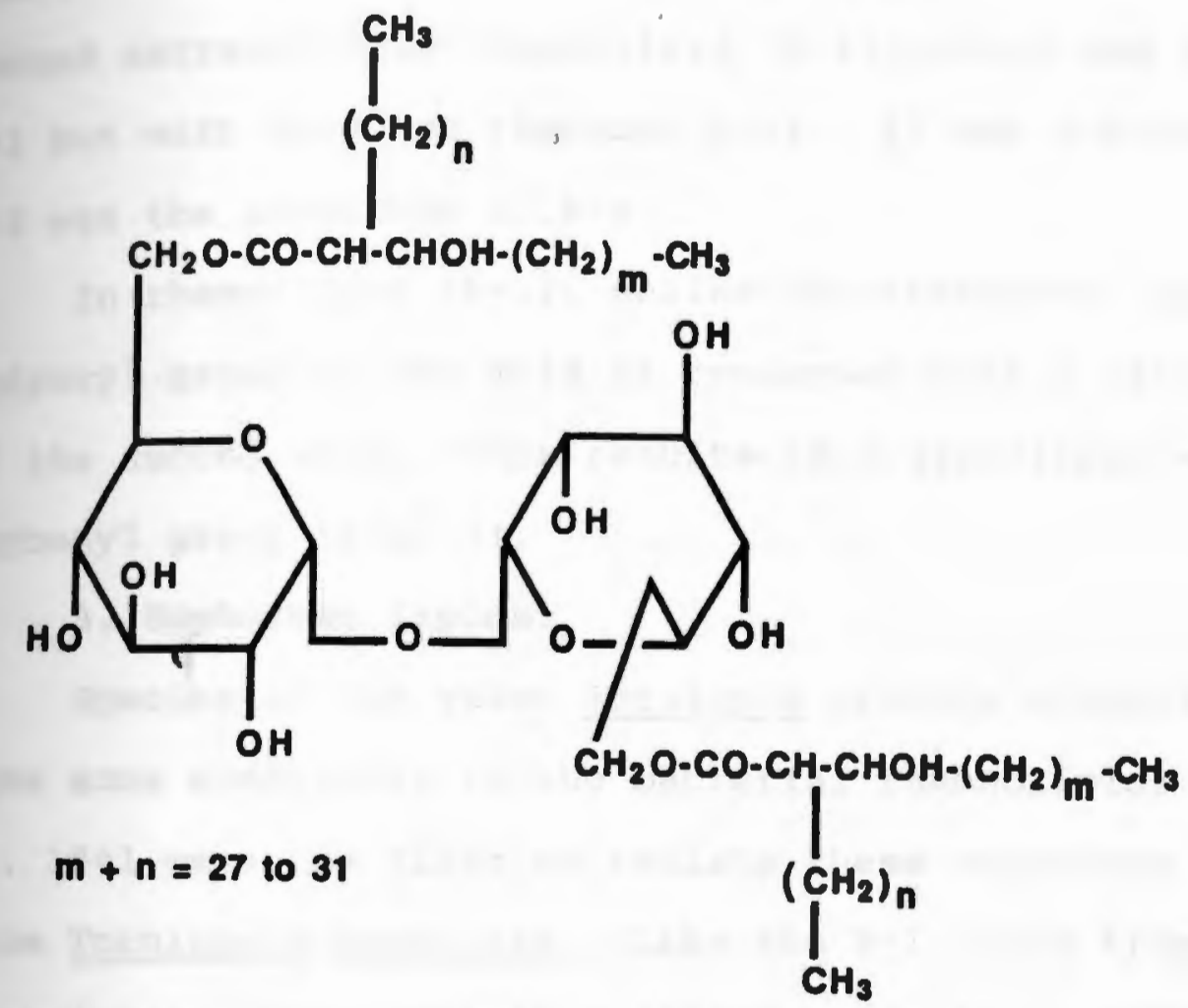

I1g.2. Trehalose-6,6'-dicorynomycolate from Rhodococcus exythropol1s 
rhamnose and two molecules of $\beta$-hydroxy-decanoic acid (Edwards and Hayashi, 1965). Itoh et al. 1971 identified a second extracellular rhamnolipid $(R-2)$, which was similar to R-1 but with only one rhamnose unit. It was postulated that $\mathrm{R}-2$ was the precursor of $\mathrm{R}-1$.

In rhamnolipid ( $R-1)$, unlike the trehalose lipids, the hydroxyl group of one acid is condensed with a carboxyl group of the second acid. This results in a glycolipid with a free carboxyl group (Fig. 3).

\section{Sophorose lipids:}

Species of the yeast Torulopis produce glycolipids that have some similarity to the bacterial rhamnolipids. Gorin et al. 1961 were the first to isolate these sophorose lipids from Torulopsis magnoliae. Like the R-1 lipid from $\underline{\text { P. }}$ aeruginosa, these contain a disaccharide (i.e. Sophorose) attached glycosidically to the hydroxyl function of a hydroxy carboxylic acid. Unlike $\mathrm{R}-1$, there is only one fatty acid and these are also one or two acetate groups attached to the sophorose (Fig. $4 a, b)$. It was found in Torulopsis that the fatty acid in the sophorose lipids was influenced by the type of addition of carboxylic acid or methyl ester, hydrocarbons, or glycerides to the culture medium.

\section{Polysaccharide-lipid complexes:}

Kaeppeli and Fiechter (1976) found a polysaccharide-lipid complex that "bound" hydrocarbon substrates of the yeast candida tropicalis. This complex, 


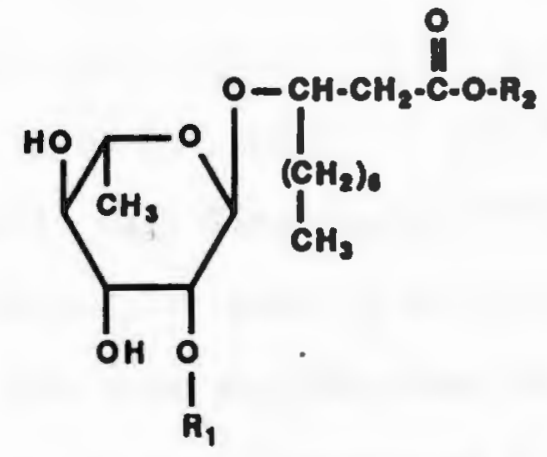

AL $1: R_{1}=L-\alpha$ - Ahamnepyranceyt-

$R_{2}=\beta \cdot$ Hydroxydecanale ecld

FL 2: $R_{1}=H$

$R_{2}=B \cdot$ Hydroxydecanole ecld

Fi.3. Two rhampose 11p1de fron Peeudomonae epecies
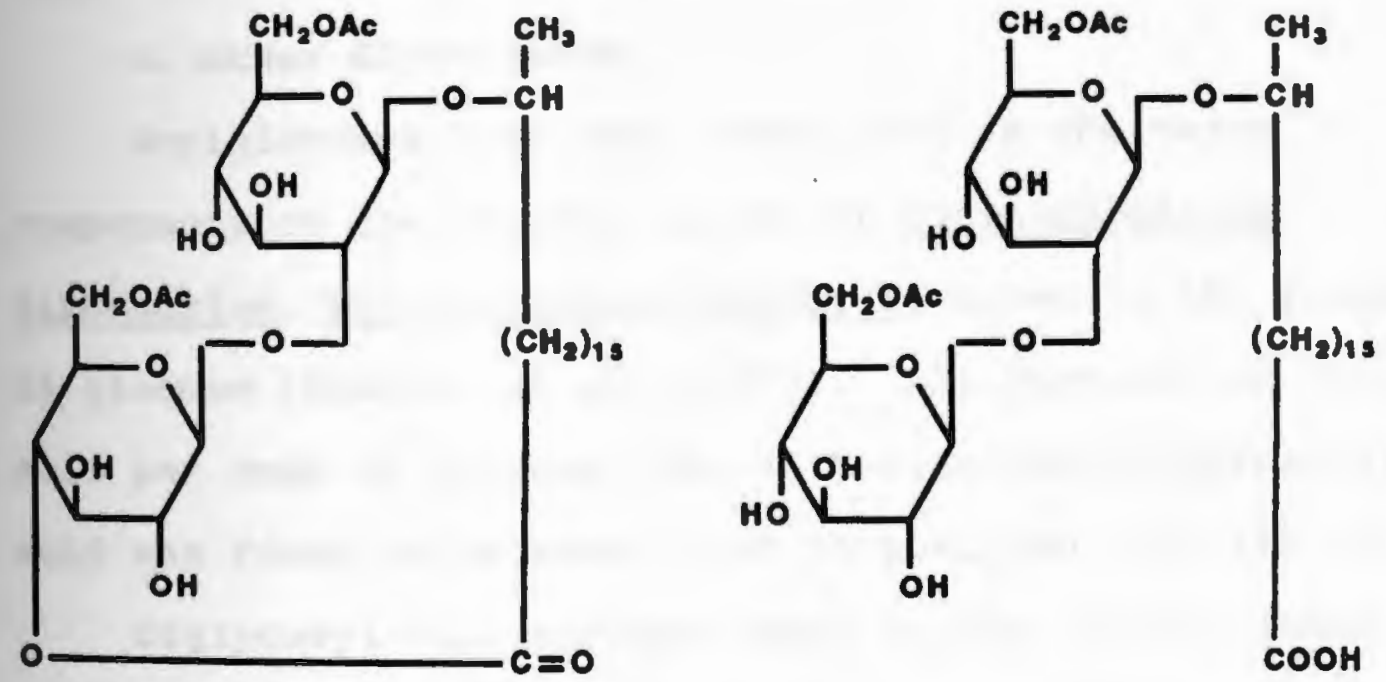

I1g.4. Iactonlc and acidic sophorose 11p1d Iron torulope1. agnoliae (bomblcola) 
isolated from the cell was found to emulsify hexadecane and water, and the lipids were found to be a mixture of saturated and unsaturated fatty acids, most of which had 14, 16, and 18 carbon atoms (Kaeppeli and Fiechter, 1977).

In the same chemical class, a surface active polymer has been reported from the hydrocarbon-degrading bacteria Acinetobacter calcoaceticus (Rosenberg et al., 1979 a, b). This polymer had a molecular weight of about $9.76 \times 10^{5}$. It contained d-galactosamine (30\%) and unidentified amino uronic acid (33\%), D-glucose (5\%), and fatty acids (15\%) (Zuckerberg et al., 1979). They also found that a wide number of oils could be emulsified with water using this polymer. This water soluble extracellular bioemulsifier which released from cell surface as a protein complex was called emulsan.

\section{Other Glycolipids:}

Acylglucoses have been identified as the major components of the soluble lipids of Corynebacterium diphtheriae, Mycobacterium smegnatis, grown in the presence of glucose (Brennan et al., 1970). All contain one fatty acid per mode of glucose, and a $\alpha$-branched- $\beta$-hydroxy fatty acid was found to be easterfied to position 6 of the glucose. Diglycosyl diglycerides shown in Fig. 5 were found to be the most common type of glycolipids found in microorganisms (Shaw, 1970). 


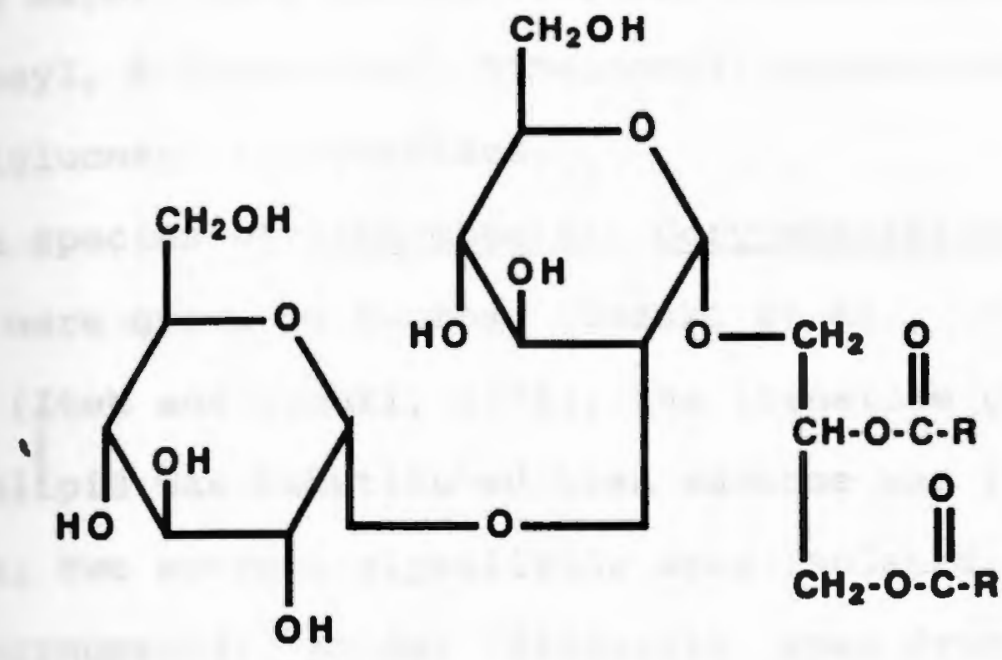

$R=$ Alkyl Substituent

I1g.5. A generalized structure of a -diglucosyldiglyceride 
Wicken and knox (1970) reported micelle formation by glycosyl diglycerides isolated from Lactobacillus fermenti. Diglycosyl diglycerides consist of a disaccharide moiety ycosidically bound to the hydroxyl group of a diglyceride.

Five major types have so far been characterized:

-diglucosyl, $\beta$-diglucosyl, dimannosyl, digalactosyl, and enctosylglucosyl diglycerides.

When species of Arthrobacter, Corynebacterium, and Nocardia were grown on sucrose (Suzuki et al., 1974); or fructose (Itoh and Suzuki, 1974), the trehalose portion of the glycolipid was substituted when sucrose was the substrate, two sucrose glycolipids were isolated, having one or two Corynomycolic acids. Similarly, when fructose was the substrate, fructose-6-Corynomycolata and fructose 1.6-dicorynomycolate were isolated.

Resting cells of Arthrobacter sp. strain DSM 2567 incubated in the presence of various mono, di, or trisaccharides biosynthesized different glycolipids. All eight glycolipids containing the corresponding carbohydrate moiety and one, two, or three $\alpha$-branched- $\beta$-hydroxy fatty acids, were produced when mannose, glucose, cellobiose, maltose, and multotriose were used as carbon sources in a simple phosphate buffer ( $\mathrm{Li}$ et al,, 1984). Incubation with monosaccharides glucose and Mannose led to one spot only on thin layer plates, which seemed to be derived from monoesters, but incubation with the disaccharides cellobiose and maltose, and the trisaccharide maltotriose led to two 
different types of spots on thin layer plates. The more polar ones seemed to be derived from monoesters, and the lipophilic ones seemed to be derived fron diesters. Thus mannose and glucose are esterified at one position only with the fatty acids, whereas cellobiose, maltose, and maltotriose are esterified at one and two position, respectively with fatty acids.

\section{B. Amino acids-Containing surfactants-}

\section{Lipopeptides:}

A lipopeptide (surfactin) produced by Bacillus subtilis ,

is the most effective biosurfactant reported in the literature (Arima et al., 1968). Its structure as shown in Fig. 6 was found to be a chain of seven amino acids covalently bonded at one end to the carboxyl function and at the other end to the hydroxyl function of a $\beta$-hydroxy fatty acid (Kakinuma et al., 1969 a, b, c).

Iguchi et al. (1969) isolated a hydrocarbon-emulsifying agent from Candida petrophilum growing on alkanes. This agent was found to contain a peptide and one or more fatty acids. The peptide was composed of aspartic acid, glutamic acid, alanine, and leucine, which is similar to the peptide fraction of surfactin.

\section{Ornithine lipids:}

Wilkinson (1972) has isolated a lipid from Pseudomonas rubescens containing only one amino acid, ornithine, which caused emulsification. The compound contained both a $\beta$-hydroxy carboxylic acid and a simple carboxylic acid 


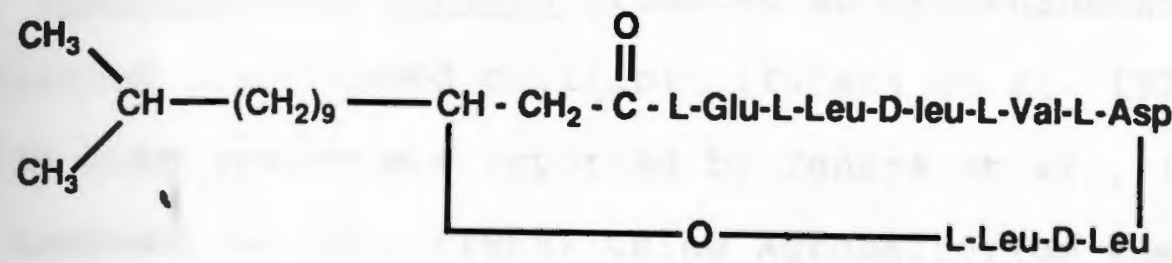

Fig.6. The atructure of aurfactin or aubtilysin, - Iipopaptide lsolated from Bacillus subtilis 
esterified to the hydroxyl function of the first acid. The $\beta$-hydroxy acid was amide linked to the $\alpha$-amino group of ornithine (Fig. 7). Another ornithine-containing lipid was obtained by Knoche and Shirely (1972) from Thiobaccilus thiooxydans. The 3-OH hexadecanoic acid was determined to be attached to the a-amino group of ornithine through an amide bond.

Gluconobacter cerinus produced an ornithine-and-taurine containing lipid named cerilipin (Tahara et al. 1976a). Lipids with lysine are reported by Tahara et al., (1976b), and Kawanami et al., (1968) using Agrobacterium tumefaciens IFO 3058 and Streptomyces sioyaensis respectively.

\section{Protein}

Hisatsuka et al., (1972) isolated a "protein-likeactivator" for n-alkane oxidation from Pseudomonas aeruginose $\mathrm{s}_{7} \mathrm{~B}_{1}$, when the organism was cultivated on $\mathrm{n}$-hexadecane. This had a molecular weight of about 14,300 and 147 amino acid residues (Histasuka et al., 1975). This activator was able to emulsify hexadecane and water, and it stimulated the growth of $\underline{P}$. aeruginosa on hexadecane (Hisatsuka et al., 1977).

Another Pseudononas strain, $\underline{\text { P. aeruginose }} \mathrm{P}-20$, assimilating $\mathrm{n}$-hexadecane, produced a peptidoglycolipid which is composed at 53 amino acid residues, a lipid moiety of 11 fatty acids and rhannose (Koronelii et al., 1983).

\section{Phospholipids:}

All contain a glycerol unit esterified to two fatty 


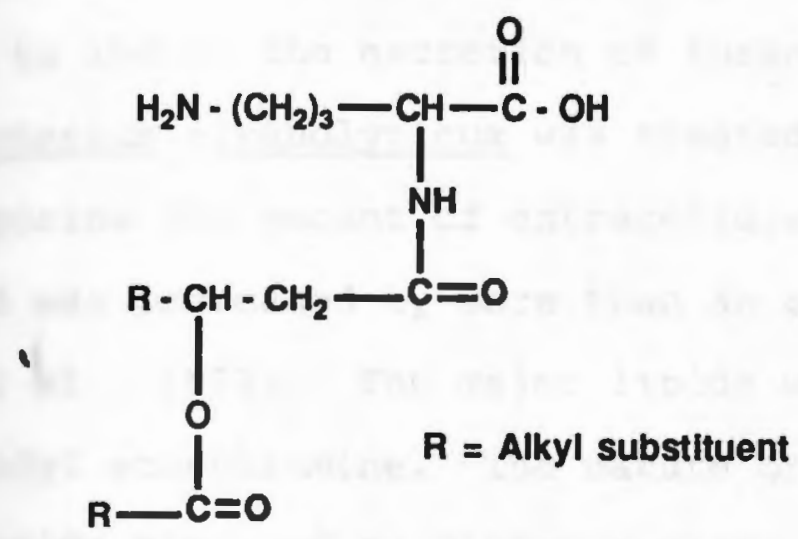

Iig.7. The structure of the ornithine -containing Ilpld 1solated from Pseudomons rubescens. 
acids and one phosphate group that may be involved in additional substitution (Fig. 8). This Bacillus thiooxydans produces various phospholipids that have been isolated from the cell free broth (Beebe and Vinbreit., 1971). Although it is relatively uncommon for microorganisms to produce appreciable amounts of extracellular phospho-lipids, it is possible to induce the excretion of these lipids. When Corynebacterium alkanolyticum was treated with penicillins or cephalosporins the amount of extracellular phospholipids recovered was increased by more than an order of magnitude (Nakao et al., 1973). The major lipids were cardiolipin and phosphatidyl ethanolamine. The nature of the mixture and phospholipids produced by microorganisms is influenced by the substrate and other growth conditions. The amount of phospholipids produced by Candida tropicalis was much larger for cells grown on n-alkanes than for cells grown on glucose (Mishina et al., 1977).

\section{Fatty Acids and Neutral lipids-}

Fatty acids and neutral lipids are found in all

microbial cells and are often produced extracellulary (Shaw, 1974). Most of these lipids, including, carboxylic acids, alcohols, esters, monoglycerides, diglycerides, and triglycerides, have been shown to have some degree of surface activity (Helenius and Simons, 1975).

Corynebacterium lepus produces a mixture of biosurfactants, when grown on Kerosene, which reduces the surface tension of the whole broth to 30 dyne/cm (Cooper et 


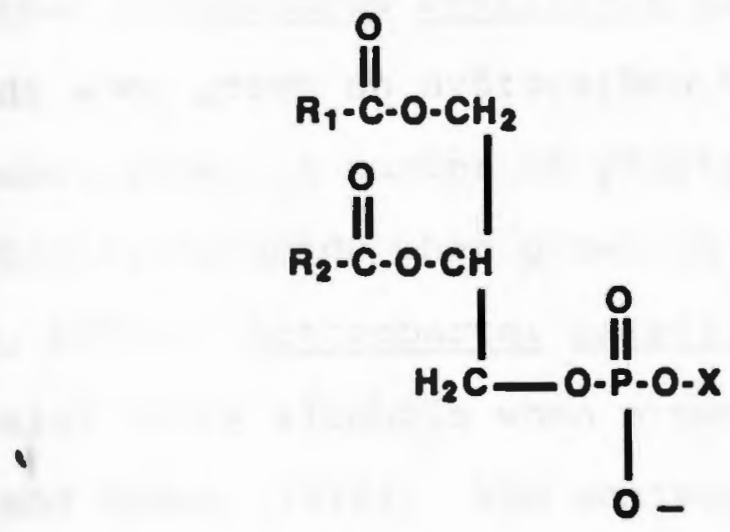

$R_{1} R_{2}=$ Alkyl substituents

$X=\mathrm{H}$ or $\mathrm{CH}_{2} \mathrm{NH}_{2}$ or $\mathrm{CH}_{2} \mathrm{CH}\left(\mathrm{NH}_{2}\right)$ COOH or Inisitol.

rig.8. General otructures of some phospholipids isolated from microorganiams. 
al., 1979a). Early in the fermentation, during the exponential growth phase, the major surfactant is a mixture of corynomycolic acids ( $\alpha$-branched- $\beta$-hydroxy Corynomycolic acid) as shown in Table 1., Makula and Finnerty (1972) observed that Micrococcus cerificans produced extracellular fatty acids when grown on hydrocarbon but not when grown on soluble substrates. A number of yeasts produce extracellular dicarboxylic fatty acids when grown on hydrocarbons (Shiio and Uchio, 1971). Arthrobacter paraffineus produces extracellular fatty alcohols when grown on hydrocarbons (Suzuki and Ogawa, 1972). The maximum concentrations were observed in the early log phase of growth. Makula et al. (1975) studied the lipids of a species of Acinetobacter grown on $\mathrm{n}$-hexadecane. The extracellular lipids isolated were mainly monoglycerides, diglycerides, and wax esters. Triglycerides and free fatty acids, were also observed as well as trace amounts of free fatty alcohols. Corynomycolic acids and other hydroxy fatty acids have been shown to be much more effective surfactants, than the simple fatty acids.

\section{E. Cell surface as a Biosurfactant:}

The cell itself can be considered to be a biosurfactant. Cell suspensions of bacteria have demonstrated surface and interfacial tension reductions, together with significant emulsification and de-emulsification activity. Factors such as culture age and broth composition affect cell 
Table 1. Corynomycolic acids isolated from corynobacterium lepus as determined by mass spectroscopy.

\begin{tabular}{cc}
28 & 2.0 \\
29 & 2.5 \\
30 & 6.8 \\
31 & 13.8 \\
32 & 21.5 \\
33 & 20.8 \\
34 & 15.0 \\
35 & 8.4 \\
36 & 4.2 \\
37 & 2.3 \\
38 & 1.3 \\
39 & 0.80 \\
40 & $\operatorname{Tr}$ \\
41 & $\operatorname{Tr}$ \\
42 & $\operatorname{Tr}$ \\
43 & $\operatorname{Tr}$ \\
\hline
\end{tabular}


Wydrophobicity (Neufeld, et al., 1980). Surface activity by atcrobial cells is due to their hydrophobic nature, and thus they can be classified as biosurfactants.

IV. Biosurfactants properties:

\section{A. Chemical properties:}

In the structure elucidation studies the glycolipids showed normal sensitivity to alkaline or acidic hydrolysis: The ester linkages were broken by saponification, the o-glycosidic bond were split by acidic condition. Other chemical reactions, like oxidations or esterification, could be carried out without difficulties. Therefore the stability of the surfactants has to be examined in each individual case depending on the field of real application. Wagner et al (1980) showed that

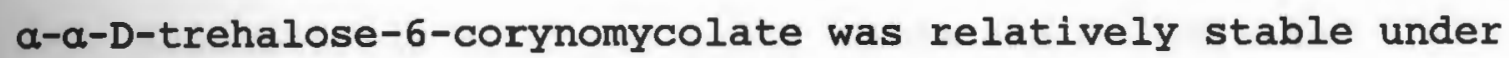
acid and alkaline hydrolysis conditions.

The surface activity of trehalose lipids synthesized by Rhodococcus erythropolis was not reduced at temperature exceeding $90^{\circ} \mathrm{C}$, and these trehalose lipids were not hydrolyzed within $96 \mathrm{hr}$ at $\mathrm{pH}$ values of 1.0 to 3.0 (Kretschmer et al., 1982).

The protein like activator of $\underline{P}$. aeruginosa $S_{7} B_{1}$ was quite stable in the $\mathrm{pH}$ range between 1.7 and 11.4 at $5^{\circ} \mathrm{C}$ and relatively stable to heat (Hisatsuka et al., 1977), they also found that, the emulsifier was partially destroyed at $98^{\circ} \mathrm{C}$ for short time, but completely inactivated after $45 \mathrm{~min}$. 
Measurements of interfacial tensions from $20^{\circ} \mathrm{C}$ up to $90^{\circ} \mathrm{C}$ in a synthetic deposite water $\left(\mathrm{NaCl} 100 \mathrm{~g} / \mathrm{L}, \mathrm{CaCl}_{2} 28 \mathrm{~g} / \mathrm{L}\right.$, $\mathrm{MgCl} / 10 \mathrm{~g} / \mathrm{L} ; \mathrm{PH} 5.5)$ showed that fructose and sucrose-6-monomycolates, the lactonic and acidic sophorose lipids, as well as the rhamnolipid with both two sugars and two fatty acids were stable at elevated temperatures (Lang et al., 1984).

B. Physical properties:

\section{Solubility:}

Nonpolar glycolipids like the nonionic trehalose-6-6-dicorynomycolates are soluble in hydrophobic solvents like chloroform; more polar glycolipids like anionic trehalose tetraesters are soluble in chloroform/methanol mixtures (Kosaric et al., 1987).

Rhamnolipids with two fatty acids are soluble in methanol, chloroform, ethylacetate, ethyl ether, and $\mathrm{NaHCO}_{3}$ solution but are not soluble in $n$-hexane (Itoh et al., 1971). Surfactin was shown to be soluble in alkaline water, methanol, ethanol, acetone, etylacetate, chloroform, and acetic acid, but to be insoluble in water, petroleum ether, and hexane. Sucrose lipids were soluble in ether, n-hexane, and chloroform at room temperature and in warm acetone and methanol. Protein-containing surfactants were soluble in water (protein like activator) or were extracted with chloroform (peptidedoglycolipid). 


\section{Surface and Interfacial Activity Properties:}

The most common property of surfactants in aqueous solution is the reduction of surface tension. The surface tension of pure water is 72 dyne/cm at $25^{\circ} \mathrm{C}$. Many biosurfactants are able to reduce the surface tension of an aqueous medium to 30 dyne/cm or lower, however, the ability at such biosurfactants to reduce interfacial tension is not directly related to the reduction of surface tension. Therefore, the interfacial tension of solution containing these compounds may vary considerably.

MacDonald et al. (1981) reported an acetone-soluble fractions (including neutral lipids and unknown glycolipid) from total lipid extraction of a Nacordia erythropolis culture, which reduced the surface tension of water to 34 $\mathrm{mN} / \mathrm{m}$ and the interfacial tension against $\mathrm{n}$-hexadecane to < $1 \mathrm{mN} / \mathrm{m}$.

It is important to evaluate the range of sodium chloride and other salt concentrations over which biosurfactants are effective at reducing interfacial tension. The surfactant properties of trehalose lipids produced by Rhodococcus erythropolis have been shown to be less sensitive to salt concentration than the common synthetic surfactants with interfacial tension remaining constant over a range of electrolyte concentrations (Kretschmer et al., 1982).

Surfactin lowered the surface tension of water to 27 $\mathrm{mN} / \mathrm{m}$ at a concentration of $0.05 \%$ (Arima et al., 1968). 
The carbon substrate on which a microorganism is grown has been shown to influence the properties of the biosurfactants produced. Glycolipid produced by isolate H13A grown on hexadecane was capable of reducing interfacial tension against decane to $0.02 \mathrm{mN} / \mathrm{m}$, whereas, an interfacial tension of $0.33 \mathrm{mN} / \mathrm{m}$ was obtained for glycolipid from heptadecane grown cells (Singer et al., 1983).

\section{Critical Micelle Concentration:}

The critical micelle concentration (CMC) represents a narrow concentration range in which a water-soluble surfactant exhibits an abrupt change in such solution properties as osmotic pressure, surface tension, viscosity, electrical conductivity and density. This change results from the formation of micelles. Any further increase in surfactant concentration above the CMC does not normally alter the interfacial tension. The CMC has frequently been reported as the dilution factor of a biosurfactant solution required to increase the surface or interfacial tension. The CMCs of many biosurfactants were considerably lower in an electrolyte solution than in distilled water (Kretschmer et al., 1982). The lower the CMC of a surfactant, the more effective it is, and the more favorable the economics of employing it in a commercial process become.

4-Emulsifying properties:

In many cases, emulsifying agents are capable of 
asiderably lowering surface and interfacial tensions. Rulsifying agent from Corynebacterium sp. reduced surface and interfacial tension to 34 and $6 \mathrm{mN} / \mathrm{m}$ respectively. Bmulsan, a heteropolysaccharide emulsifying agent produced by rcinetobacter calcoaceticus, is very effective at stabilizing emulsions (Rosenberg et al., 1979). It has been put to commercial use in cleaning crude oil storage tanks and a potential role for enhanced oil recovery (EOR) has been proposed (Basta., 1983). Biosurfactants with strong emulsifying properties may find applications as cleaning agents or in pipelining coal slurries and heavy oils; it is the reduction of interfacial tension rather than emulsifying ability which should be used as a screening criterion for the selection of biosurfactants for EOR.

\section{v. Production of Biosurfactants:}

\section{A. Growth Conditions for Biosurfactants Production:}

The biosynthesis of biosurfactants by growing cells is often connected with foam formation, a lowering of the surface and interfacial tension of the medium and an emulsion of the lipophilic substrate in the culture broth.

When it is known whether the surface active agents are cell wall-bound or secreted into the medium by the microorganisms, and a suitable procedure for product recovery has been found, the influence of different physiological and physical parameters on the metabolic activity of the picroorganism should be examined. It is important to optimize biosurfactant production during cultivation of the 
teroorganism (Kosaric et al., 1987). This may be achieved initially by optimizing nutrient requirements and growth conditions for biosurfactant synthesis, and in longer term by mplementing agenetic strain improvement program aimed at increasing product yield.

\section{Nutritional Requirements:}

Biosurfactants which are frequently produced on wydrocarbon substrates, may also be generated from carbohydrate substrates. The use of carbohydrates for large-scale biosurfactant production offers economic advantages, fermentable sugars are cheaper than hydrocarbons and fermentation costs are reduced since more power is required in a fermenter to achieve adequate disperson of hydrocarbon than is required for a carbohydrate. The type and quantity of biosurfactant produced by a microorganism has been shown to be greatly affected by the nature of the substrate. The glycolipid produced by isolate H13A grown on heptadecane differed in fatty acid composition from glycolipid produced on hexacane (Singer et al., 1983).

A strong correlation existed between the structure of the lipids of Nocardia sp. and the growth substrate (MacDonald et al., 1981).

The production of biosurfactant by Bacillus subtilis was much greater on a glucose substrate than in nutrient broth (Cooper et al., 1981).

Pseudomonas sp. produced twice as much rhamnolipid when grown on n-alkanes than on glycerol (Wagner et al., 1984). 
Nitrogen limitation has been shown to be an important factor governing biosurfactant production from both carbohydrate and ardrocarbon substrates (Guerra-santos et al., 1984).

Decreasing the nitrogen concentration to the point at which it become growth limiting for Pseudomonas aeruginosa greatly increased surfactant production (Gueera-santor et al., 1984).

A high carbon-to-nitrogen ratio led to increases in the production of rhamnolipid by Rhodococcus erythropolis; conversion of n-alkanes into rhamnolipid increased from 15 to $28 \%$ when nitrogen became limiting (Wagner et al., 1984).

The nitrogen source also influences biosurfactant production; nitrate for instance, is superior to ammonium as a nitrogen source for rhamnolipid producion by Pseudomonas aeruginosa (Wagner et al., 1983).

The concentration of certain inorganic salts influence biosurfactant production. Concentrations of iron sulfate exceeding $10 \mathrm{mg} / \mathrm{L}$ decreased biosurfactant production by Pseudomonas aeruginosa, and the yield of rhamnolipids from $\underline{P}$. aeruginosa was increased by seven-to-ten fold by optimizing nutrient requirements (Guerrasantos et al., 1984). Thus, the manipulation of growth conditions may affect the structure of the biosurfactant or greatly improve it's yield.

\section{Growth Phase:}

Different biosurfactant compounds can be identified at different stages during the growth cycle in batch cultures 
of many microorganisms. A fatty alcohol reached a maximum concentration in the early log phase of growth of Nocardia erythropolis, and then decreased in concentration towards the end of the fermentation (MacDonald et al., 1981).

An early increase in surfactant concentration in cultures of Corynebacterium lepus was attributed to the moduction of corynomycolic acid (Cooper et al., 1979), and the surface activity at the end of this fermentation was due to a lipopeptide containing corynomycolic acids plus hospholipids and neutral lipids.

3. Environmental Conditions:

The environmental conditions are another important factor which can influence surfactant production. When looking for an optimal surfactant production, it must be considered that biosurfactant synthesis and the growth of the aicroorganisms often have different optima of temperature and $\mathrm{pH}$ value. The $\mathrm{pH}$ value played an important role in biosurfactants production and in all cases the yield of biosurfactant was temperature-dependent (Kosaric et al., 1987).

B. Methods for biosurfactants production:

1. Cell Growth-Associated production of Biosurfactants: Looking at the optimal C-source for biosurfactant production, lipophilic substrate are necessary for induction of surfactant biosynthesis. The C-source plays an important role for rhamnolipid production by Pseudomonas sp. (Syldatk et al. 1985). Different C-sources such as glycerol, glucose, 
ethanol, and others could be used for rhamnolipid production by pseudomonas sp., but all were inferior to n-alkanes.

In some cases the chain length of the hydrocarbon substrates used had an effect on biosurfactant production. The formation of emulsifying and surface active agents by porynebacterium hydrocarboclastus were optimal with linear alkanes of the chain length $c_{12}, c_{13}$, and $c_{14}$, and mehaloselipid formation by Rhodococcus erythropolis was dependent on the chain length of the hydrocarbon substrate as well (Rapp et al., 1977).

Some examples are found in the literature, where different C-sources were used for growth of the microorganisms and production of the surface-active compounds; Arthrobacter paraffineus was grown on glucose and produced surface-active agents by adding hexadecane to the medium in the stationary phase of growth (Duvnjak et al., 1982). Also Torulopsis bambiocola, grown on glucose, produced large amounts of a glycolipid by addition of vegetable oils to the growing cultures in the late exponential phase of growth (Cooper and Paddock, 1984). The N-source or N-limitation plays an important role in the production of some biosurfactants which are produced in the stationary phase of growth. There are a few examples in the literature where the nature of the $\mathrm{N}$-source or an addition of $\mathrm{N}$-sources to the culture broth influences surfactant production by growing cells. The surfactant production by Arthrobacter paraffineus is increased when different amino acids, such as 
spartic acid, asparagine, glycine, or glutamie acid, were present in a mineral salts medium (Duvnjak et al., 1983), also, yeast extract, peptone, and nutrient broth had a positive effect on biosurfactant production. When inorganic salts were used as the $\mathrm{N}$-source, the organism preferred ammonium ion to nitrate. With various Corynebacteria, nitrate as sole $\mathrm{N}$-source caused surfactant production during the mid-exponential phase of growth, while nitrate plus ammonium resulted in growth-associated surfactant production (Gerson and Zajic, 1979).

The nutrient composition of the culture medium influenced surfactin production by Bacillus subtilis. The addition of iron or manganese salts to the culture broth Improved the yield of the lipopeptide (Cooper et al., 1981). In case of Rhodococcus erythropolic a mineral salts medium was optimized for surfactant production by the addition of $0.02 \%$ yeast extract (Margaritis et al., 1979).

In addition to the C-source, the $\mathrm{N}$-source, and medium composition, the environmental conditions are another Important factors which can influence surfactant production by growing cells. The $\mathrm{pH}$ value played an important role in thamnolipid production by Pseudomonas sp. (Wagner et al., 1984), and in sophorolipid formation by Torulopsis bombicola (Gabbert et al., 1984). The temperature was of great Important in the case of Arthrobacter paraffincus surfactant production (Duvnjak et al., 1982), Rhodococcus erythropolis and Pseudomonas sp. DSM 2874 (Wagner et al., 1984). In the 
case of pseudomonas sp. DSM 2874 the product composition was also dependent on the temperature. Concerning the agitation speed, the surfactant production optimum is dependent upon the agitation speed. Increase of the agitation speed from 250 to $500 \mathrm{rpm}$ caused a decrease in surfactant production by Rodococcus erythropolis because of the effect of the shear rate on the growth kinetics of the microorganism (Margaritis, et al., 1979). Cellobiose-lipid production by Ustilago Zeae (Roxburgh, et al., 1954), and Sophorolipid production by different yeast species were increased by higher agitation speeds and aeration rates (Spencer, et al., 1979), both of which resulted in higher rates of oxygen transfer. A connection between oxygen consumption rate and surfactant production was also observed in the case of Corynebacterium lepus (Crerson and Zajik 1979).

2. Biosurfactant production by growing cells under Growth-limiting conditions:

In some cases an overproduction of biosurfactants was dependent on growth-limiting conditions such as N-limitation or limitation of the multivalent cations; the rhamnolipid overproduction by Pseudomonas aeruginosa occurred only after reaching the stationary phase of growth (Hauser and Karnovsky, 1953). The limitation of $\mathrm{NH}_{4}^{+}$or $\mathrm{NO}_{3}^{-}$ions induced a rhamnolipid overproduction by Pseudomonas sp. DSM 2874 during batch cultivation using n-alkanes as c-source and caused a change in crude product composition (Syldatk et al., 1985). Rhamnolipid formation by Pseudomonas aeruginosa 
in continuous culture, grown on glucose as C-source, was Increased seven to tenfold after $\mathrm{NO}^{-}{ }_{3}$ limitation (Suzuki et al., 1974). By limitation of $\mathrm{Fe}^{2+}, \mathrm{Mg}^{2+}$, or $\mathrm{Ca}^{2}$ cations, which are all essential for the growth of Pseudomonas, a further increase in rhamnolipid production was observed (Guerra-Santos et al, 1984).

When Rhodococcus erythropolis was grown under non-limiting growth conditions, only non-ionic trehalose corynomycolates were formed. After $\mathrm{N}$-limitation a dramatic change in the production composition was observed, so that anionic trehalose tetraesters were formed (Ristau and Wagner., 1983). This was also observed in the case of Corynebaterium hydrocarboclactus and Arthrobacter paraffineus (Restau et al., 1983).

From these results it is supposed that the effect of N-limitation or a limitation of the multivalent cations is non-specific and it is expressed as a change of the physiological state of the microorganism used for biosurfactant production (Kosaric et al. 1987).

3. Biosurfactant production by Resting Cells:

It is possible to examine the microbial production of secondary metabolites, which are produced in the stationary phase by non-dividing cells or by resting cells. For resting cell experiments microorganisms are separated from the culture broth by centrifugation after cultivation under optimal growth conditions. The wet biomass is washed and suspended in a non-growth solution such as buffer for the 
Hoduction of secondary metabolites under specific conditions, so that the effect of possibly disturbing byproducts can be eliminated and the influence of single factors on the synthesis of the compound (such as $\mathrm{pH}$ value, temperature, different cations, salt concentration, etc.) can be examined.

This method was successfully applied to rhamnolipid formation by resting free or resting immobilized cells of Pseudomonas sp. DSM 2874 (Syldatk et al. 1984), sophorolipid production by resting free cells of Torulopsis bombicola (Gobbert et al., 1984), cellobiose lipid production by resting free cells of Ustilage maydis (Frantz and Wagner, 1984), and trehalose tetraester production by resting free cells of Rhodococcus erythropolis (Wagner et al., 1984).

In the case of Arthrobacter sp. DSM 2567 it is possible to incubate resting free cells in the presence of various mono-di- or trisaccharides to obtain the corresponding glycolipids (Li, et al., 1984).

The highest yield of rhamnolipids from n-alkanes as C-source was obtained when resting free cells of Pseudomonas sp. DSM 2874 were incubated in phosphate buffer or sodium chloride solution at a pH value of 6.6 and a temperature of $37^{\circ} \mathrm{C}$ (Syldatk et al., 1985).

Looking at the syphorolipid production by Torulopsis bombicola from glucose as c-source, there was hardly a difference between cells in the stationary phase of growth and resting cells in sodium chloride solution (Gabbert et 
al., 1984), where the production of sophorolipids was optimal at $\mathrm{pH} 3.5$ and $22^{\circ} \mathrm{C}$.

The addition of $\mathrm{Mg}^{2+}$ and $\mathrm{Fe}^{2+}$ cations to the culture broth of resting cells of Ustilago maydis was essential for Mlobioselipid formation (Frautz and Wagner, 1984). The optimal conditions for collobioselipid production were $\mathrm{pH} 6.6$ and $33^{\circ} \mathrm{C}$.

Resting cells of Rhodococcus erythropolis showed a distinctly higher conversion rate ( $g m$, trehalose tetraester/gm substrate) than growing cells under $\mathrm{N}$-limitation.

4. Biosurfactant production by microbial cells and addition of precursors:

There are some examples in the literature for biosurfactant production by microbial cells after addition of precursors, so that it is possible to increase the yield of biosurfactants by addition of lipophilic compounds to the culture broths as in the case of sophorolipids formed by Terulopsis magnoliae (Tulloch et al., 1962), or various glycolipids formed by Torulopsis bombiocola (Cooper and Paddock, 1984). Further the nature of the glycolipids produced can be directed by using a specific carbohydrate as the C-source. Growing on such a c-source the microorganisms produces the corresponding glycolipid. Glucose, fructose, and sucrose lipids produced by Arthrobacter paraffineus and several Corynebacterium, Nocardia, and Brevebacterum species were examples for this pathway of glycolipid production 
(Itah and Suzuki, 1974; Suzuki et al., 1974).

This method of biosurfactant production will probably be of great interest in future because it allows the production of new surfaces and interfacially active compounds whereby the chemical and physical properties of these compounds can be influenced by the C-sources used for glycolipid formation.

VI. Isolation and purification of Biosurfactants: Concerning different methods of biosurfactant recovery, there are many methods applicable to the isolation of these compounds. The method used for the isolation of a certain product depends on the nature of this compound (whether it is water-soluble or not, an ionic or nonionic, cell wall-bound or extracellular). Therefore, there are no general rules for the isolation of biosurfactants. A suitable method or a combination of suitable methods for product recovery has to be developed for every new isolated compound. Most of the biosurfactants are lipophilic compounds and can be isolated by "classic" methods such as extraction, precipitation, or crystallization.

With regards to the isolation of glycolipids from microbial cultures, generally it is to distinguish between cell wall-bound and extracellular compounds. The glycolipids are mostly excreted into the medium when they have an ionic character. After separating the cells by centrifugation, many glycolipids such as cellobiose lipids produced by Ustilago species (Frautz and Wagner, 1984), 
phoralipids produced by Torulopsis and Candida species (Cooper and Paddock, 1982; Gobbert et al., 1984), sucrose, fructose, and trehalose lipids produced by Arthrobacter paraffineus (Itoh and Suzuki, 1974; Suzuki et al., 1974; Ii et al., 1984), trehalose corynomycolates and tetraesters produced by Rhodococcus erythropolis (Ristau and Wagner, 1983; Rapp et al., 1979; Kretschmer et al., 1982; MacDonald, et al., 1981), and rhammolipids produced by Pseudomonas species (Hauser and Karnovsky, 1954; Yamaguchi et al., 1976; Hisatsuka et al., 1971), can be isolated by extracting either the cell mass or the supernatant with different organic solvents as ethyl acetate, ether, pentane, hexane, butanol, chloroform-methanol, or dichloromethane-methanol. After solvent removed by rotary evaporation, the crude product can be purified by adsorption column chromatography on silicic acid, thick-layer chromatography on silica gel (preparative thin-layer chromatography), and crystallization.

In some cases, glycolipids can be obtained by acidifying the supernatants, with hydrochloric or surfuric acid to $\mathrm{pH}$ 2.0-3.0 and refrigerating for some time. This is possible in the case of rhammolipids (Hauser and Karnovsky, 1954), pllobioselipids (Boothroyd et al., 1955), and some btosurfactants produced by Corynebacterium hydrocarboclastus (Duvnjak et al., 1982). The glycolipids can be isolated as precipitate or as crystals from the supernatant. A precipitation of biosurfactant is also possible in the case 
of protein like compounds such as emulsan (Rosenberg et al., 1979), or a protein emulsifier produced by Torulopsis trophilum (Cooper and Paddock, 1982). Here a precipitation is achieved by the addition of ammonium sulfate to the supernatants. The subsequent purification procedure includes adsorption on ion exchange chromatography resins, dialysis and concentration.

Iipids with one amino acid were isolated by the method of Bligh and Dyer (1959), homogenzing the wet biomass after separation from the supernatant with a mixture of chloroform and methanol. After the addition of water the chloroform phase contained all the lipids.

Lipids with more amino acids (e.g. surfactin, a lipid with seven amino acids) are isolated as follow: After elimination of cells the $\mathrm{pH}$ value of the filtrate was adjusted to $\underline{2}$ with hydrochloric acid; the resulting precipitate was dissolved in alkaline water. After addition of $\mathrm{CaCl}_{2}$ the $\mathrm{Ca}$ precipitation was resuspended in hydrochloric and newly generated acid precipitate was extracted with diethyl ether (Arima et al., 1968). After evaporation and dissolving the residue in acetone, decolorizing by active charcoal and sephadex LH 20 elution, surfactin was crystallized from acetone/water.

Lipopeptides other than surfactin were extracted from the culture filtrate with n-butanol, for example, and the chromatographed on silica gel. 
In the case of n-alkane fermentation the cell separation is too difficult, therefore the extraction method is favored over precipitation method.

Besides these classic methods, which cannot be used for a continuous product recovery during cultivation, there are some methods for biosurfactant recovery which can be called "in situ recovery." By using these methods it is possible to remove the products continuously from the culture broth during the cultivation.

In the case of surfactin produced by Bacillus subtilis ' (cooper et al., 1981) and some surfactants formed by Clostridium pasteurianum (Cooper et al., 1980) the products can be isolated by flotation and foam fractionation. The foam, which contains the products, is collapsed after flotation by acid precipitation and extracted with different solvents.

Another method of in situ product recovery is the adsorption of surfactants to ion exchange resins or other suitable absorbents as in the case of lipopeptide surfactant produced by Candida petrophilum (Iguchi et al., 1969) or hammolipids produced by Pseudomonas sp. DSM 2874 (Syldatk et al., 1984). So it is possible to avoid an end product inhibition as well as foam problems during the cultivation. Another example for an in situ recovery of surfactants is the in situ extraction of cell wall-bound compounds from prynebacterium lepus by addition of alkanes to the culture broth of this microorganism (Duvnjak and Kosaric, 1981). 
arfactants produced by this microorganism can be completely removed from the cells in 2-4 hr by addition of dodecane or hexadecane to the culture medium.

In situ methods for product recovery will probably become of increasing interest in future because they avoid end product inhibition during fermentation, reduce costs of solvents and waste water treatment, and minimize product degradation (Raffler et al., 1984).

VII. Structure elucidation of Biosurfactants:

When a pure glycolipid has been obtained, proved by thin-layer chromatography and followed by detection with sugar and lipid specific reagents the substance is hydrolyzed under alkaline and acidic conditions. Alkaline hydrolysis splits the ester bond between the fatty acid group and the $\mathrm{OH}$ group of the sugar. O-Glycosidic linkages between two sugars and between a sugar and a hydroxyl group of a lipid moiety can be broken by acid hydrolysis.

When a saponification only leads to water-soluble pure sugar and water-unsoluble lipid moieties, this fact confirms the ester bond. If two or more sugars are combined, the location of glycosidic binding can be proven by either periodate oxidation or ${ }^{13} \mathrm{C}$-NMR spectroscopic studies. The structure of monosaccharide and disaccharide can be determined by comparison with authentic samples using thin-layer, gas, and high pressure liquid chromatography or by enzymatic proof. For the structure elucidation of the 
fatty acid, the combination of ${ }^{1} \mathrm{H}-,{ }^{13} \mathrm{C}-\mathrm{NMR}$, and gas chromatography/mass spectroscopy is very helpful (Kosaric et al., 1987).

When alkaline saponification of a natural glycolipid results in a fatty acid and a new, more hydrophilic lycolipid, this fact points to an ester bond and an Cglycosidic bond between a sugar and a hydroxyl group of a lipid moiety.

In the work of syldatk et al. (1985), resting cells of Pseudomonas SP. DSM 2874 were incubated at $30^{\circ} \mathrm{C}$ in $\mathrm{NaCl}$ solution yielding two glycolipids and two novel rhamnolipids. The substances were separated by thick-layer chromatography. For structure elucidation TLC, IR, ${ }^{1} \mathrm{H},{ }^{13} \mathrm{C}-\mathrm{NMR}, \mathrm{GC}-\mathrm{MS}$, and HPLC were employed. Alkaline hydrolysis didn't lead to fatty acid because no ester function was present, and that is evidence for only one fatty acid present ( $\alpha$ branched $\beta$-hydroxy fatty acid).

In the studies of Rapp et al., (1979), the lipid moiety of the trehalose lipid was characterized by ${ }^{13} \mathrm{C}-\mathrm{NMR}$ and MS indicating predominantly saturated long-chain $a$-branched $\beta$-hydroxy fatty acids, ${ }^{13} \mathrm{C}-\mathrm{NMR}$ analysis of the 0 -hexamethyl trehalose obtained by saponification of the premethylated trehalose dicorynomycolates revealed, with the aid of deuterium exchange, that the ester linkages are to both alcohol groups at the $c-6$ and $c-6$ positions of the trehalose. 
Sucrose lipids were isolated from Arthrobacter raffineus KY 4303 when the microorganism was grown on surcose (Suzuki et al., 1974). By mild acid hydrolysis it was demonstrated that the ester bond of sucrose lipid was located at the glucose residue because only fructose was detected as free sugar-periodate oxidation experiments auggested that the fatty acid, a corynomycolic acid, might bind to the 6-position of glucose. In the case of fructose the corresponding fructose-6-corynomycolatus were formed (Itoh and Suzuki, 1974).

Resting cells of Arthrobacter sp-DSM 2567 incubated in the presence of various mono- di-, or trisaccharides Blosynthesized different glycolipids ( $\mathrm{Li}$ et al., 1984). The structures of all eight glycolipids were elucidated by means of ${ }^{1} \mathrm{H}-$ and ${ }^{13} \mathrm{C}-\mathrm{NMR}$ and by chemical ionization mass spectroscopy .

The location of the acyl groups on the sugar molecule is a problem that can be attacked in different ways. One way is the irreversible blocking of the free hydroxyl groups of the sugar moiety by methylation. Then the pre-methlated glycolipid is hydrolyzed in acid medium, giving poly-o-methyl monosaccharide. The places where the fatty acids were linked to the sugar moiety in the initial glycolipid are pointed out by the alcohol groups that remain free in the methylated sugar. Another way begins with the reversible protection of the free hydroxyl groups by acetalation (dihydropyrane) and continues with deacylation 
of the ester groups in alkaline medium. After methylation of these few hydroxyl groups the other hydroxyl groups that had been protected by acetalation were liberated by acid adrolysis. Now places where fatty acids were linked to the earbohydrates moiety in the initial glycolipid are labeled by the o-methyl groups. A third way to determine the location of acyl groups is the application of the spectroscopic methods, including ${ }^{13} \mathrm{C}-\mathrm{NMR}$ spectroscopy (Kosaric et al., 1987).

To determine the structure of amino acid-containing lipids, mild alkaline hydrolysis was performed resulting in a fatty acid and deacylated amino acid-containing lipid. Acid Mydrolysis using $\mathrm{HCl}(6 \mathrm{M})$ at $105^{\circ} \mathrm{C}$ for $12-16 \mathrm{hr}$ of this lipid gave the amino acid shown by an amino acid analyzer, and a second fatty acid mixture the $\mathrm{C}-3$ hydroxy group of which is esterified with the carboxylate of the first fatty acid. The location of the $\mathrm{OH}$ group of the fatty acid was confirmed by combined gas chromatography/mass spectrometry in the case of ornithin-containing lipid (Wilkinson, 1972).

Lipopeptides like surfactin were treated first with $\mathrm{HCl}$ leading to a mixture of amino acids and fatty acids. The resulting amino acids were estimated by an amino acid analyzer (Kakinuma, et al., 1969). The amino acid sequence was determined using the method by Edman (1950) with modifications to use trifluoro-acetic acid in hydrolysis and to analyze the amino acids in peptides remaining after Mydrolysis. The fatty acid structure was studied by $1_{\mathrm{H}-\mathrm{NMR}}$ 
and

iss-spectroscopy, that the fatty acid was bound to the dimethyl-N-acyl glutamate as a methanolysis product of warfactin (Kakinuma et al., 1969). The higher the molecular weight the more difficult is it to elucidate the structure of Mosurfactants; in the case of protein activators of emulsifiers only the amino acids and carbohydrate moieties were analyzed after hydrolysis.

Sometimes the molecular weight could be estimated by gel hromatography.

\section{Application of Biosurfactants:}

The application of biotechnology in the surfactant industry represents a tremendous potential and opens challenging avenues. The surfactant industry is fast growing within the U.S. chemical industry, increasing by over $300 \%$ between 1972 and 1982 (Katzenberg, 1985).

The initial focus and most of the industrial interest in blosurfactant has been toward microbial production of surfactants, co-surfactants, and so on for microbial-enhanced oil recovery (MEOR) (Donaldson and Clark, 1983).

It should be noted that a biopolymer produced by Coryneform sp. H13A has the ability to lower the interfacial tension to $2 \times 10^{2}$ dynes/cm. with the addition of pentanol as a co-surfactant the interfacial tension was reduced to $6 \mathrm{x}$ $10^{-5}$ dynes/cm (Anonyomous, 1983). This biosurfactant, 
which is a glycolipid, could be very competitive with present omercial EOR surfactants.

It is of industrial interest to develop the mourfactant production in which cost of the raw material and the process becomes minimal. only if this requirement is met and the price of biosurfactants becomes lower than that of petroleum and lignosulfonates do biosurfactants have a chance in being used on a large scale for this application (Kosaric et al., 1987).

Besides MEOR, another area where biosurfactants can be used successfully in the control of oil spills. For this application sulfonates are major competitors. However, biosurfactants may have the advantage of their being active dispersants and emulsifiers at the same time as their being biodegradable.

The only commerical industrial biosurfactant on the market presently is that of Emulsan, patented by (Gutnick et al., 1983). The "emulsans" ( $\alpha$ and $\beta$ ) are extracellular microbial protein-associated lipopolysaccharides. Emulsan is presently being marketed by petroleum fermentation (Petroferm) for use in cleaning oil contaminated vessels, oil spill management, and MEOR (Sittig and Noyes, 1985). In addition to the interest by petroleum industry, losurfactants can find interesting applications in a number of other industries, such as agriculture, food and beverage, cleaning, paint and protective coating, pharmaceuticals, cosmetics, and textiles industries (Kosaric et al., 1987). 
An indirect important of biosurfactants is that they facilitate the growth of microorganisms on hydrocarbons and a large number of useful products such as single-cell protein (Mateles et al., 1968), as, well as a large number of individual amino acids, alkane transformation products, sugars and polysaccharides, nucleic acids, vitamins, and pigments (Abbott and Gledhill, 1971), can be obtained from these substrates (Cooper and Zajic, 1980). 
CHAPTER II

\section{MATERTALS AND METHODS}

All of the chemicals used were of technical grade. Deionized water was used throughout because of the severe Influence of trace elements on microorganism growth and - ssurfactant production.

I. Organism and growth conditions:

Isolation of the microorganisms

Arthrobacter (JIZAN-I) was isolated from the nvironment. Five petri dishes containing nutrient agar (BBL Div-Becton, Dickinson Company, Cockeysville, MD), exposed to air for 15 min. at different locations outside Woodward Hall, University of Rhode Island in summer of 1986 , were then covered and incubated (Blue M Bacteriological Incubator, Blue Island, IL), at $30^{\circ} \mathrm{C}$ for 2 days.

A loopful of JIZAN-I was transferred into sterilized $250 \mathrm{ml}$ Erlenmyer flasks containing $100 \mathrm{ml}$ filter sterilized basal mineral salts medium with $200 \mathrm{mg}$ glucose as the sole carbon and energy source covered with $50 \mathrm{ml}$ beakers and incubated on a Psycrotherm incubator-shaker (New-Brunswick Scientific Co., Inc., New Brunswick, NJ) at $200 \mathrm{rpm}$ and $30^{\circ} \mathrm{C}$. After the appropriate incubation period, growth (turbidity) was measured in Klett summerson photoelectric colorimeter (Model 800-3, Klett KFG Co., Inc., N.Y.), then the culture was centrifuged (Sorval RC-5B refrigerated centrifuge, Dupont Instruments, Norwalk, CT) at 10,000 rpm for $10 \mathrm{~min}$. to separate the cells from the culture broth. 
surface and interfacial tensions of the supernatant cell-free culture), were measured by using Fisher atotensiomate (Fisher Scientific, Inc., Medford, MA).

After the isolation of JIZAN-1 and examination of its capability for producing biosurfactant, working stock cultures were prepared on nutrient agar slants, incubated at $30^{\circ} \mathrm{C}$ for $15 \mathrm{hr} .$, and stored for up to one month at $4^{\circ} \mathrm{C}$. For long term storage, the stock culture was maintained at $-70^{\circ} \mathrm{C}$ in 28 glycerol.

4

\section{Modified basal mineral salts medium}

The basal mineral salts medium has been described by Traxler and Bernard (1969) table 2. The composition of the modified basal mineral salts medium was as follows (concentration per liter of deionized water): $1000 \mathrm{mg}$ of $\mathrm{Na}_{2} \mathrm{HPO}_{4} \cdot 7 \mathrm{H}_{2} \mathrm{O}, 500 \mathrm{mg}$ of $\mathrm{KH}_{2} \mathrm{PO}_{4}, 1500 \mathrm{mg}$ of $\mathrm{NH}_{4} \mathrm{NO}_{3}, 500 \mathrm{mg}$ of $\mathrm{MgSO}_{4}, 7 \mathrm{H}_{2} \mathrm{O}, 20 \mathrm{mg}$ of $\mathrm{CaCl}_{2} 2 \mathrm{H}_{2} \mathrm{O}, 1 \mathrm{mg}$ of $\mathrm{FeSO}_{4} \cdot 7 \mathrm{H}_{2} \mathrm{O}$, $10 \mathrm{mg}$ of $\mathrm{MnSO}_{4} \cdot \mathrm{H}_{2} \mathrm{O}, 5 \mathrm{mg}$ of $\mathrm{Co}\left(\mathrm{NO}_{3}\right)_{2} \cdot 6 \mathrm{H}_{2} \mathrm{O}$, and $100 \mathrm{mg}$ of $\left(\mathrm{NH}_{4}\right)_{6} \mathrm{MO}_{7} \mathrm{O}_{24} \cdot 4 \mathrm{H}_{2} \mathrm{O}$.

Glucose was used as the sole carbon and energy source in concentration of $2000 \mathrm{mg} / \mathrm{L}$, and yeast extract was used in a concentration of $200 \mathrm{mg} / \mathrm{L}$ as a growth cofactor.

\section{Cultivation Conditions}

A loopful from the slant culture of JIZAN-1 was transferred into a $250 \mathrm{ml-Erlenmyer} \mathrm{flask} \mathrm{or} \mathrm{side} \mathrm{arm} \mathrm{flask}$ pontaining $100 \mathrm{ml}$ filter sterilized modified basal mineral salts medium containing $200 \mathrm{mg}$ glucose as carbon and energy source and $20 \mathrm{mg}$ yeast extract, the flask was covered with 
Table 2. Composition of the basal mineral salts medium (BMSM) .

\section{Components} .

$\mathrm{Ha}_{2} \mathrm{HPO}_{4} \cdot 7 \mathrm{H}_{2} \mathrm{O}$

$\mathrm{KH}_{2} \mathrm{PO}_{4}$

$\mathrm{NH}_{4} \mathrm{NO}_{3} 1$

$\mathrm{MSSO}_{4} \cdot \mathrm{TH}_{2} \mathrm{O}$

- $\mathrm{CaCl}_{2} \cdot 2 \mathrm{H}_{2} \mathrm{O}$

$\mathrm{FeSO}_{4} \cdot 7 \mathrm{H}_{2} \mathrm{O}$

$\mathrm{MnSO}_{4} \cdot \mathrm{H}_{2} \mathrm{O}$

$\mathrm{Co}\left(\mathrm{NO}_{3}\right)_{2} \cdot 6 \mathrm{H}_{2} \mathrm{O}^{1}$

$\left(\mathrm{MH}_{4}\right)_{6} \mathrm{MO}_{7} \mathrm{O}_{24} \cdot 4 \mathrm{H}_{2} \mathrm{O}^{-1}$

\section{Amount}

(mg/l distilled $\mathrm{H}_{2} \mathrm{O}$ )

1000

500

2500

500

20

1

10

5

100 
$50 \mathrm{ml}$ beaker and incubated on a Psycrotherm at $200 \mathrm{rpm}$ and $30^{\circ} \mathrm{C}$.

\section{Growth measurement}

After the appropriate incubation period, growth was measured by two methods:

1. Cell growth was monitored by measuring the turbidity in Klett units by using Klett-Summerson photoelectric solorimeter.

2.Measurement of growth in terms of cell mass (Biomass) was determining using a known volume of fermentation culture which was centrifuged at 15,000 rpm for $15 \mathrm{~min}$., the pellet was suspended in distilled water and recentrifuged. The dry weight of this biomass was determined by weighing after drying at $105^{\circ} \mathrm{C}$ for $24 \mathrm{hr}$.

\section{Cell separation}

Since the biosurfactant was extracellular, after the appropriate incubation period for growth, the suspension was centrifuged (Sorvall RC-5B refrigerated superspeed centrifuge Dupont Instrument, Norwalk, CT) at 10,000 rpm for $10 \mathrm{~min}$. to separate the cells from the culture broth. 
Fig. 9. Isolation scheme of Arthrobacter JIZAN-1, and examination its ability to produce biosurfactant from water-soluble substrate (glucose) as c-source. 


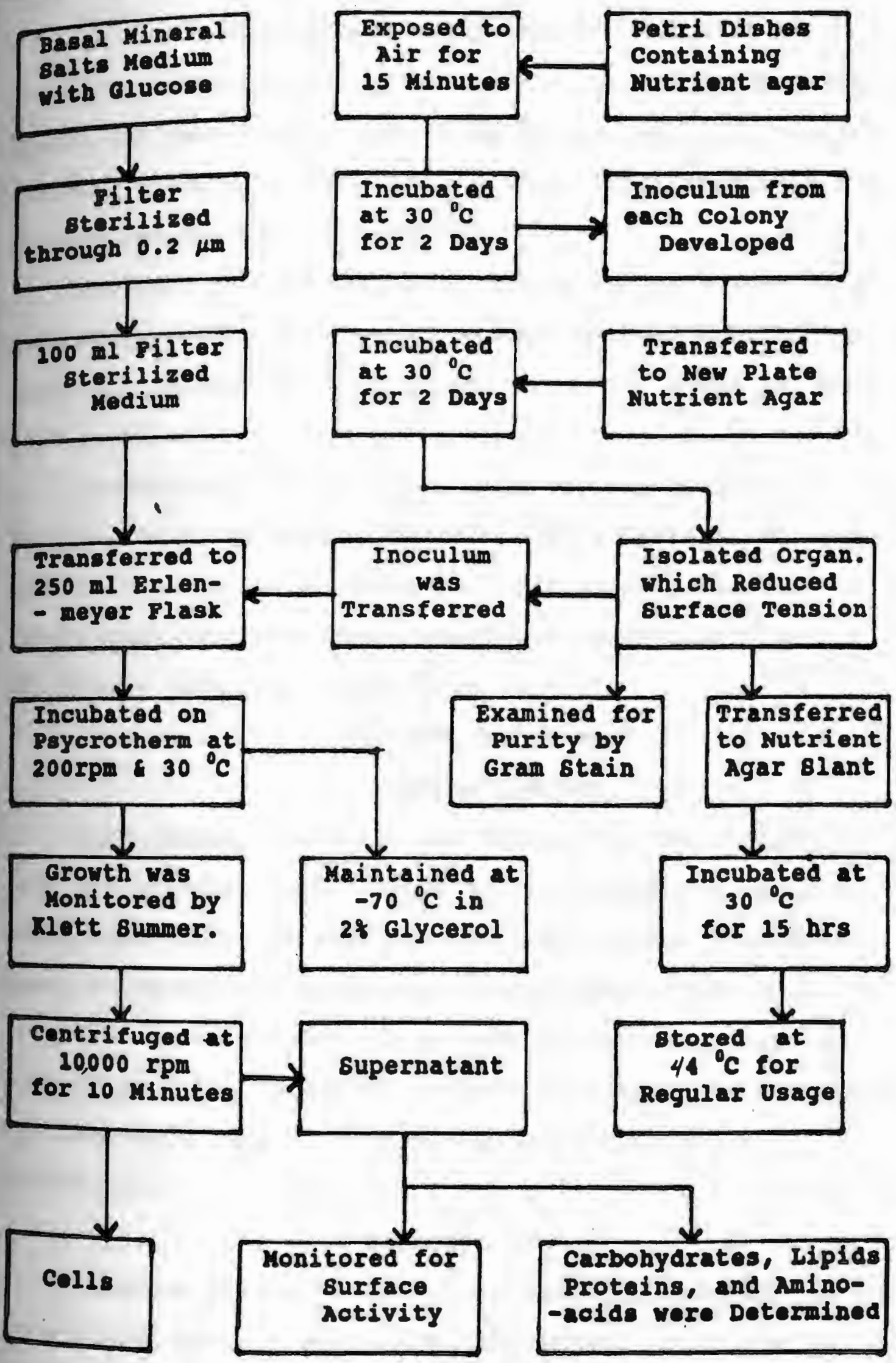




\section{Preliminary analytical methods}

After centrifugation to separate the cells from culture broth, the supernatant (cell-free medium) was analyzed for vesidual glucose, and surfactant activity by measuring the surface and interfacial tensions.

Residual glucose concentration of the cell-free culture was determined by the glucose oxidase method in a Glucose analyzer (Yellow Spring Instrument Co., YSI Model 27, Yellow spring, Ohio).

A modified duNouy surface tensiometer "Fisher atotensiomat" (Fisher Scientific, Inc., Medford, MA) was used to measure the surface and interfacial tensions. All Interfacial tensions measurement were made using fresh interfaces (less than $3 \mathrm{~min}$.) at $25 \pm 3^{\circ} \mathrm{C}$.

\section{"Growth Conditions"}

\section{Carbon Sources}

Arthrobacter (JIZAN-I) was tested for the ability to grow and produce biosurfactant at the expense of various carbon sources which included glucose, starch, hexadecane, sweet crude oil, \#2 heating oil, and Kuwait crude oil.

Glucose as C-source in a range of $100-400 \mathrm{mg} / 100 \mathrm{ml}$ with $50 \mathrm{mg} / 100 \mathrm{ml}$ increment was used to determine the optimal c-source concentration for growth and biosurfactant production.

\section{Nitrogen Sources}

Ammonium nitrate $\left(\mathrm{NH}_{4} \mathrm{NO}_{3}\right)$ and ammonium chloride $\left(\mathrm{NH}_{4} \mathrm{Cl}\right)$ were tested as nitrogen sources for growth and biosurfactant 
poduction by strain JIZAN-1. Ammonium nitrate $\left(\mathrm{NH}_{4} \mathrm{NO}_{3}\right)$ and anmonium chloride $\left(\mathrm{NH}_{4} \mathrm{Cl}\right)$ were tested in the range of 50-350 $\mathrm{mg} / 100 \mathrm{ml}$ (at $50 \mathrm{mg} / 100 \mathrm{ml}$ increment) and used or separately as $\mathrm{N}$-sources to determine the optimal $\mathrm{N}$-source concentration for growth and production of biosurfactant.

\section{Environmental Conditions}

The effect of temperature range between $20-35^{\circ} \mathrm{C}$ (at $5^{\circ} \mathrm{C}$ Increments) was used to determine the optimum temperature for growth and biosurfactant production.

In order to determine the effect of initial medium $\mathrm{pH}$ on growth and biosurfacant production, the medium was poised at $0.5 \mathrm{pH}$ units over the range of $\mathrm{pH} 4.0$ to 7.5 and inoculated with standardized inoculum.

\section{Growth Phase}

To establish the relationship between growth phase and surfacant production, $50 \mathrm{ml}$ aliquots were collected at increasing turbidity ( 25 Klett Unit intervals) between 50 and 250 Klett Units.

\section{Identification of the Microorganism:}

The identification of the microorganism was based on worphological, cultural, and biochemical tests, as outlined in Bergey's Manual of Determinative Bacteriology (Buchannam and Ribbons, 1974).

\section{A. Gram stain reaction:}

The morphological characteristics of the microorganism were determined by utilizing gram stain reaction, by using 15 hrs. culture grown on nutrient agar. 


\section{B. Gelatin hydrolysis:}

A $15 \mathrm{hr}$. culture from nutrient agar was streaked across the center of a nutrient gelatin plate (nutrient agar $+4 \%$ (elatin),

incubated at $30^{\circ} \mathrm{C}$ for $24 \mathrm{hr}$. The plate was flooded with acidified $\mathrm{HgCl}_{2}\left(15 \mathrm{HgCl}_{2}, 20 \mathrm{ml} \mathrm{HCl}\right.$, make to $\left.100 \mathrm{ml}\right)$ to precipitate the unhydrolyzed gelatin.

A positive reaction consisted of a clear zone aurrounding the growing bacterium.

c. Starch hydrolysis:

A $15 \mathrm{hr}$. culture from nutrient agar was streaked across the center of a nutrient starch agar plate (Bacto starch agar plate), incubated at $30^{\circ} \mathrm{C}$ for $24 \mathrm{hr}$. The plate was flooded with iodine solution "Lugol's solution" (1 gm iodine, $2 \mathrm{gm} \mathrm{KI}$, and $300 \mathrm{ml} \mathrm{H}_{2} \mathrm{O}$ ), which turns the unhydrolyzed starch blue.

A positive reaction consisted of a colorless zone surrounding the growing bacteria.

\section{Glucose fermentation:}

The production of acid and gas during glucose fermentation by the microorganism was tested by using the (Bacto phenol red broth $+1 \%$ glucose) medium.

A loopful from a culture grown on nutrient agar in slant at $30^{\circ} \mathrm{C}$ for $15 \mathrm{hr}$., was used to inoculate the Bacto phenol red $+1 \%$ glucose medium in a test tube, then incubated at $30^{\circ} \mathrm{C}$ for $24 \mathrm{hr}$. 
Acid production was indicated by a yellow color change of phenol red acid-base indicator. Gas production was detected by using Durham fermentation tube, which indicates fermentative metabolism.

\section{B. Nitrate reduction to Nitrite:}

The reduction of nitrate to nitrite by the Microorganism was tested by using Bacto nitrate broth. The composition of the test reagent for nitrite was sulphonilic acid, $8 \mathrm{~g} / \mathrm{L}$ in $5 \mathrm{~N}$ acetic acid, and naphthyl amino acetate, 5 $\mathrm{g} / \mathrm{L}$ in $5 \mathrm{~N}$ acetic acid.

A loopful from a culture grown on nutrient agar in slant at $30^{\circ} \mathrm{C}$ for $15 \mathrm{hr}$. was used to inoculate the Bacto nitrate broth. The culture was incubated at $30^{\circ} \mathrm{C}$ for $24 \mathrm{hr}$ then $1 \mathrm{mI}$ of each reagent added to the culture. The positive reaction consisted of a red color formation due to the presence of nitrite.

\section{F. Anaerobic Growth test:}

Anerobic growth of the microorganism was tested in reinforced clostridial agar by (BBL) by a stab inoculation from a $15 \mathrm{hr}$. nutrient agar slant, then incubated at $30^{\circ} \mathrm{C}$ for $24 \mathrm{hr}$. Growth along the line of inoculation to the bottom of the stab indicates anaerobic growth.

\section{G. Motility test:}

The motility of the microorganism was tested in Bacto motility test medium (Semi-solid media) by stab inoculation in the center of the media, then incubation at $30^{\circ} \mathrm{C}$ for 24 
hr. Motility is indicated by diffuse horizontal growth from the line of inoculation.

III. Determination of proteins, lipids and carbohydrates excreted into the culture broth during the fermentation process.

\section{Proteins}

Proteins excreted into the culture broth during the fermentation process were determined by folin phenol reagent methods of Lowry et al. (1951) based on bovine albumin as the standard. The Klett Summerson photoelectric colorimeter was used for measuring the intensity of the color in Klett units using the red filter.

\section{Lipids}

Lipids excreted into the culture broth during the fermentation process were determined by utilizing three techniques:

\section{Lipid extraction:}

The class of extraction process called continuous liquid-liquid extraction was applied for separation the lipids from the culture broth by using the organic solvent diethyl ether.

Lipids, the oily residual which remains in the flask after complete evaporation of diethyl ether using vacuum evaporator, were used for identification by TLC and for fatty acids determination by GC. 


\section{Identification of Lipids by TLC:}

Thin-layer chromatography (TLC) technique was used for separation and identification of lipids which extracted by diethyl ether. A Silica gel G type TLC plate was employed as the stationary phase. Two lipid standards were used, one contained polar lipids, and the other contained the nonpolar lipids. TLC plate was first developed by disopropyl ether and acetic acid $(96 / 4)$ for $2-3 \mathrm{~cm}$ up from the base line, then dried with nitrogen and rechromatographed in petrolum ether, diethyl ether and acetic acid $(90 / 10 / 1)$ to near the top of the plate. The plate was dried and exposed to iodine vapours for visualizing compounds with double bonds on TIC (Kates, 1972).

3. Determination of fatty acids by gas chromatography: Three major steps were applied for this purpose:

a. Saponification:

Lipids which were extracted by diethyl ether were wydrolyzed by using $0.5 \mathrm{~N}$ potassium hydroxide ( $\mathrm{KOH}$ ) in methanol, to cleave the natural ester bonds between fatty acids and glycerol.

\section{b. Derivatization}

The free fatty acids which resulted from the hydrolysis of lipids were methylated (converted to their methyl esters) by using borontrifluoride $\left(\mathrm{BF}_{3}\right)$ in methanol.

c. Fatty acids analysis by gas chromatography:

The resultant fatty acid methyl esters were analyzed and the distribution of fatty acids was determined by gas 
chromatography (Gunstone., 1967).

Non-volatile long chain fatty acids $\left(C_{16}-C_{24}\right)$ :

The non-volatile long chain fatty acid methyl esters

were analyzed on Varian aerograph series 1700 gas

chromatograph (Varian Company, CA) equipped with flame

Ionization detector and cyanosilicon (10\% sp-2330) column.

Two standards. GLC-10 and PUFA-1 were used for determination

of the relative retention times.

Non-volatile short chain fatty acids $\left(\mathrm{C}_{\mathbf{8}}-\mathrm{C}_{16}\right)$ :

The non-volatile short chain fatty acid methyl esters were analyzed on Hewlett-Packard series 5730A gas

chromatograph (Avondale, PA) equipped with flame ionization

detector and 5\% DEGS (diethyl glycol succinate) column. Two

standards GLC-30 and GLC-70 were used for determination the

relative retention times.

Volatile acids $\left(C_{2}-C_{7}\right)$ :

Volatile acids were analyzed on a Hewlett, Packard

series $5730 \mathrm{~A}$ gas chromatograph equipped with flame

ionization detector and $10 \% \mathrm{SP}-1200$ with $1 \% \mathrm{H}_{3} \mathrm{PO}_{4}$ column. A

mixture of $\mathrm{C}_{2}-\mathrm{C}_{7}$ acids was used as the standard for

determination of the relative retention times.

\section{Carbohydrates}

Carbohydrates in the culture broth were determined by atilizing two different methods:

1. Colorimetric methods:

Reducing sugars excreted into the culture broth were cetermined by anthrone-sulfuric acid method (Dreywood, 1946) 
and by phenol-sulfuric acid method (Dubois et al. 1956) based on glucose as the standard. Two instruments were used for measuring the intensity of the color formed: Klett gummerson photoelectric colorimeter with red filter (Model 800-3, Klett MFG Co., Inc. N.Y.) and Lambda 4B UV/VIS trophotometer (PERKIN-ELMER, Lambda 4B uv/VIS trophotometer, Norwalk, CT) with wavelength $485 \mathrm{~nm}$ for the color developed by phenol-surfuric acid.

2. Gas chromatographic method:

Three steps was applied for determination of carbohydrates which excreted into the culture broth during the fermentation process by (GC).

\section{a. Hydrolysis:}

The hydrolysis procedure of Adams (1965) was modified and used as follows:

1. $200 \mathrm{ml}$ of the lipid free culture broth was evaporated to dryness, then $10 \mathrm{ml}$ of $72 \%$ sulfuric acid $\left(\mathrm{H}_{2} \mathrm{SO}_{4}\right)$ were added and held at room temperature for $45 \mathrm{~min}$.

2. The mixture was diluted with $200 \mathrm{ml}$ distilled $\mathrm{H}_{2} \mathrm{O}$, and autoclaved for $45 \mathrm{~min}$. at $121^{\circ} \mathrm{C}$, then neutralized with IN NaOH and evaporated to dryness.

3. $100 \mathrm{ml}$ of $80 \%$ ethanol were added to the dried mixture and aged overnight, the filtered and evaporated to dryness.

\section{b. Derivatization:}

The derivatization procedure and Sweeley et al., (1963) was modified as follows: 
1. One ml of "STOX" reagent (Hydroxylamine containing phenyl-B-D-glucopyranoside as the internal standard, Pierce Chemical Co., Rockford, IL) was added to $30 \mathrm{mg}$ of the dried mixture, then heated for $30 \mathrm{~min}$ at $70-75^{\circ} \mathrm{C}$.

2. One $\mathrm{ml}$ of hexamethyldisilazane (HMDS, Pierce chemical Co., Rockford, IL) and $0.1 \mathrm{ml}$ trifluoroacetic acid (TFAA) were added to the oxime formed in the previous step, then shaken for $20 \mathrm{sec} .$, and allowed to stand at room temperature for $30 \mathrm{~min}$.

c. Gas chromatographic analysis of sugars:

one $\mu l$ of the resultant trimethylsilyl derivatives were injected into a temperature programmed gas chromatograph (Hewlett, Packard Series 5730A Gas Chromograph, Avondale, PA) equipped with Flame ionization detector and ov-17 column (phenyl methyl silicone, Supelco, Inc., Bellefonte, PA). The GC was connected to chromatopac C-R3A data processor with thermal sensitive printer plotter (Shimadzu Corporation, Kyoto, Japan). Starting temperature which was held for $2 \mathrm{~min}$. after injection was $150^{\circ} \mathrm{C}$, the final temperature which also held for $2 \mathrm{~min}$. was $260^{\circ} \mathrm{C}$, and the temperature program rate was $8^{\circ} \mathrm{C} / \mathrm{min}$. (Al-Hazmi et al., $1985)$.

The sugars and sugar derivatives ribose, rhamnose, xylose, fructose, glucose, galactose, glucuronic acid, galactonic acid, and galacturonic acid, were used as standards for determination the relative retention times. 
IV. Blosurfactant production by continuous fermentation:

A. Inoculum preparation:

A loopful from a 15 hour culture grown on nutrient agar was transferred to $250 \mathrm{ml-Erlenmyer} \mathrm{flasks} \mathrm{containing} \mathrm{a} 100$ ml filter sterilized modified basal mineral salts medium with $200 \mathrm{mg}$ glucose and $20 \mathrm{mg}$ yeast extract, then incubated on a New Brunswick Psycrotherm (New Brunswick Scientific Co., Inc., New Brunswick, NJ) shaker at $200 \mathrm{rpm}$ and $30^{\circ} \mathrm{C}$ for $12 \mathrm{hr}$. This culture served as inoculum for the following continuous fermentation.

\section{B. Experiment \#1}

Carbon limited continuous fermentation:

A $1000 \mathrm{ml}$ modified filter sterilized basal mineral salts medium containing $200 \mathrm{mg} / 100 \mathrm{ml}$ glucose as carbon source, $150 \mathrm{mg} / 100 \mathrm{ml}$ ammonium chloride $\left(\mathrm{NH}_{4} \mathrm{Cl}\right)$ as nitrogen source, and $20 \mathrm{mg} / 100 \mathrm{ml}$ yeast extract were transferred under strict sterilization conditions into sterilized $2 \mathrm{~L}$ Bioflo model (C-30) fermentor (New Brunswick Scientific Co., Inc., New Brunswick, NJ), then inoculated with the $100 \mathrm{ml}$ cell suspension (Inoculum prepared in $\underline{A}$ ). The fermentation cell suspension was controlled at $\mathrm{pH} 7.0$, temperature at $30^{\circ} \mathrm{C}$, and the agitation was $200 \mathrm{rpm}$. The $\mathrm{pH}$ controller was used to maintain the $\mathrm{pH}$ at 7.0 by the addition of $2 \mathrm{~N} \mathrm{NaOH}$ and $1.5 \mathrm{~N} \mathrm{HCl}$.

Continous fermentation was initiated when the culture reached the early log growth (130 KU), by the addition of fresh modified filter sterilized basal mineral salts medium 
antaining $200 \mathrm{mg} / 100 \mathrm{ml}$ glucose, $150 \mathrm{mg} / 100 \mathrm{ml}$ ammonium chloride $\left(\mathrm{NH}_{4} \mathrm{Cl}\right)$, and $20 \mathrm{mg} / 100 \mathrm{ml}$ yeast extract to the fermentor. The flow rate of the fresh medium into the fermentor was maintained at $2.0 \mathrm{ml} / \mathrm{min}$ and constant culture volume in the fermentor $(1100 \mathrm{ml})$ was maintained by using a continous pump attached to a draft flow tube at specific vessel volume $\left(D=0.11 \mathrm{hr}^{-1}\right)$.

Foam was controlled electrically to add as needed antifoam $A$ concentrate solution which is (active silicone polymer without emulsifiers) was utilized for foam uppression.

Cell density was determined by measuring the turbidity of the culture broth by using Klett summerson photoelectric colorimeter (modle 800-3, Klett KFG Co., NY), and the biomass was determined by passing a $10 \mathrm{ml}$ sample of the broth through a prewashed, preweighed Nuclepore membrane filter $\left(0.2 \mu \mathrm{m}\right.$ pore size). This sample was dried at $105^{\circ} \mathrm{C}$ and reweighed.

Cells were separated from the cell suspension by centrifugation at 10,000 rpm for $10 \mathrm{~min} .$, using (Sorvall CC-5B, Dupont Inst., Norwalk, CT) centrifuge.

Residual glucose in the (cell-free medium) culture broth was determined by glucose oxidase. Ammmonium concentration in the culture broth was determined by orion Model 901 Microprocessor with ammonium electrode.

Surface and interfacial tensions were determined by using Fisher autotensiomat (Fisher Scientific Co., Inc., 
Wedford, MA).

\section{c. Experiment \#2:}

same as experiment \#1 except the $\mathrm{N}$-source was ammonium nitrate $\left(\mathrm{NH}_{4} \mathrm{NO}_{3}\right)$ and the amount of glucose was $300 \mathrm{mg} / 100$ m1. Continuous fermentation was initiated when the culture reached $175 \mathrm{kU}$ turbidity.

v. Isolation and purification of the biosurfactant: The biosurfactant purification were carried out through three stages:

\section{A. Extraction of the biosurfactant:}

The organic solvents chloroform, ethyl acetate, petrolum ether, hexane, benzene, and various combinations of each two solvents at different ratios were employed to extract the biosurfactant from (cell-free medium) culture broth at different temperatures. Multiple extraction using separatory funnel was applied for extraction. The ability of the solvent to extract the biosurfactant was examined by measuring the surface tension of broth, the culture broth after extraction, and the extract from which the organic solvent was removed by rotary evaporation (Flash-evaporator, Fort Lee, NJ), and redissolving the residue in distilled water.

\section{B. Separation by Thin-layer chromatography (TLC) :}

The chloroform extract obtained by multiple extraction of the culture broth at $35^{\circ} \mathrm{C}$, was separated by TLC plate. Silica Gel G type TLC plates were employed as tationary phase for separation of the chloroform extract. 
Fig. 10. Extraction and purification scheme for AL-Hazmi surfactant, in which a combination of two steps were utilized (silica gel column and $c_{18}$ silica column) for purification.

$\mathrm{A}=$ Hexane $=$ Chloroform: ethyl acetate: Methanol $(30: 40: 20: 10)$.

$B=100 \%$ Methanol.

C = Hexane: Chloroform: ethyl acetate: Methanol $(20: 30: 20: 30)$

D = Chloroform: Methanol (60:30) 


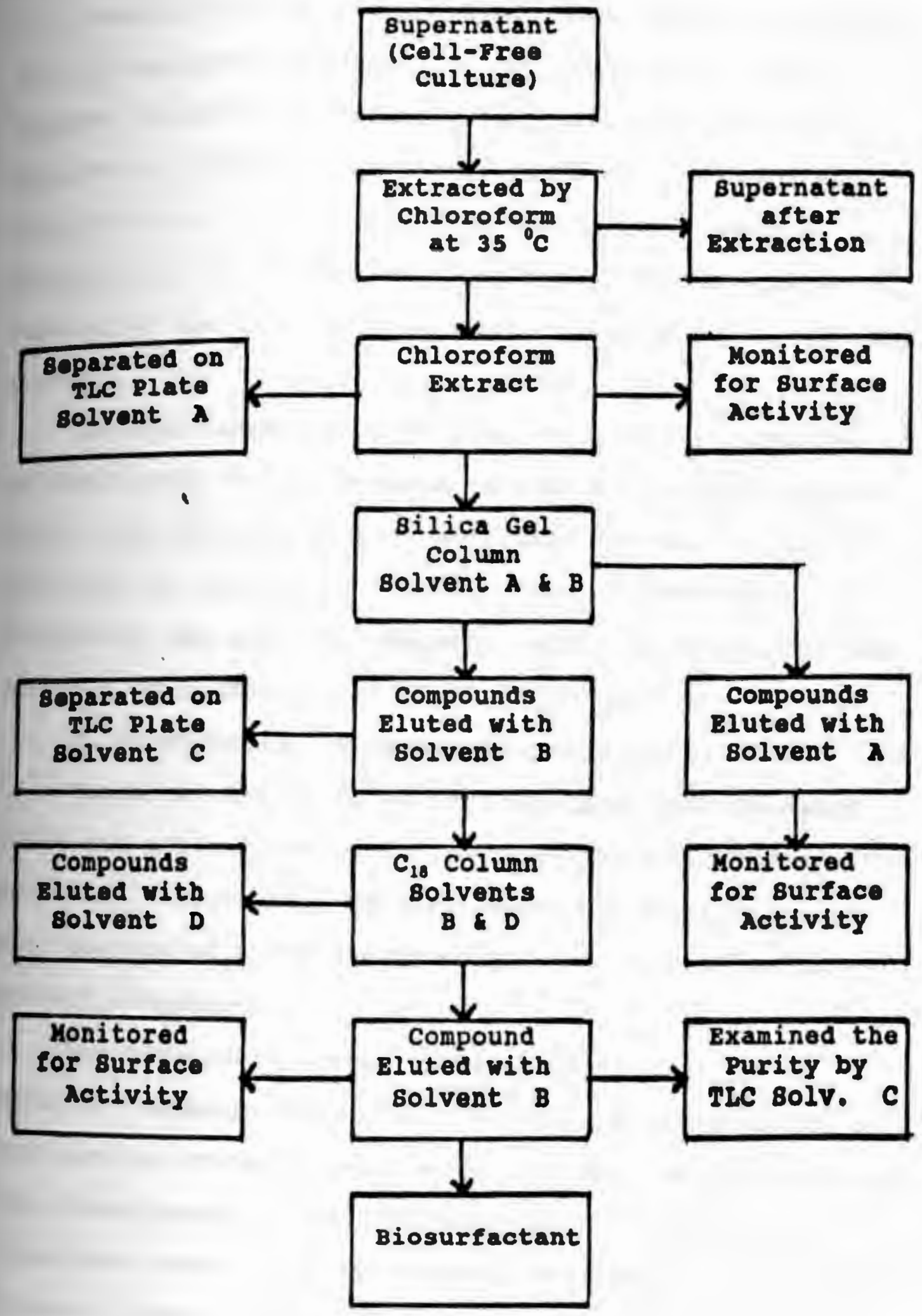


Several combinations of HPLC grade organic solvents such as chloroform: Hexane (50:50), chloroform: ethyl acetate $(50: 50)$, chloroform: ethyl acetate: methanol (50:20:30), Hexane: chloroform: ethyl acetate: methanol (30:40:20:10), and hexane: chloroform: ethyl acetate: methanol (20:30:20:30), were applied as mobile phases for separation the chloroform extract on silica gel G type TLC plates.

several reagents (detectors) such as $0.2 \%$ anthrone in sulfuric acid, $0.2 \%$ ninhydrin in acetone, iodine vapours, UV light, and vanillin reagent were used for visualizing the compounds on TLC plates. In the case of anthrone, ninhydrin, and vanillin reagents, spots on TLC plates were detected after heating at $100^{\circ} \mathrm{C}$ for $10 \mathrm{~min}$.

\section{c. Purification by column chromatography:}

Flash-chromatography column equipped with threaded joint and solvent reservoir (Aldrich Chemical Company, Inc., Malwaukee, Wisconsin), were employed for separation and purification of the biosurfactant from other chloroform extract components.

The stationary phases, silica gel, silicic acid, sephadex, sephacryl, florosil, alumina, and $\mathrm{C}_{18}$ silica gel were used as column packing materials for purification of the biosurfactant. The HPLC grade organic solvents hloroform, ethyl acetate, hexane, acetone, petrolum ether, methanol, and a combination of these solvents at various ratios were applied as a mobile phases for purification of 
the biosurfactant by column chromatography.

A fraction collector (ISCO, Model 400 volumeter) was used to collect 5 or $7 \mathrm{ml}$ fraction, and each fraction or group of fractions were collected separately and concentrated by evaporation of the organic solvents using rotary evaporator, then spotted on TLC for examination the purity.

vI. Identification of the biosurfactants:

A. Chenical methods:

After the purification by open column chromatography, and proof of purity by thin layer chromatography (TLC), the substance was hydrolyzed under alkaline and acidic conditions.

1. Alkaline hydrolysis:

Biosurfactant was hydrolyzed under alkaline condition through heating by using $0.5 \mathrm{~N}$ potassium hydroxide $(\mathrm{KOH})$ 1nmethanol. The reaction mixture was cooled, acidified to less than $\mathrm{pH} 3.0$ with $1 \mathrm{~N} \mathrm{HCl}$, extracted with ether and separated into an ether-soluble fraction containing fatty acids and a water phase containing a glycoside. Fatty acids in the ether-soluble fraction were derivatized by borontrifluoride $\left(\mathrm{BF}_{3}\right)$ in methanol, then the resultant fatty acid methyl esters were analyzed and the distribution of fatty acids were determined by a varian aerograph series 1700 gas chromatograph (Varian Company, CA) equipped with flame ionization detector and $10 \% \mathrm{sp} .2330$ (cyano silicone) column. Several fatty acid methyl ester mixtures including 
Fig. 11. Structure eludication scheme of AL-Hazmi surfactant, in which chemical methods including hydrolysis under alkaline and acidic conditions and physical methods were utilized for examination of AL-Hazmi surfactant structure.

$\mathrm{C}=$ Hexane $=$ chloroform: ethyl acetate $:$ methanol $(20: 30: 20: 30)$ 


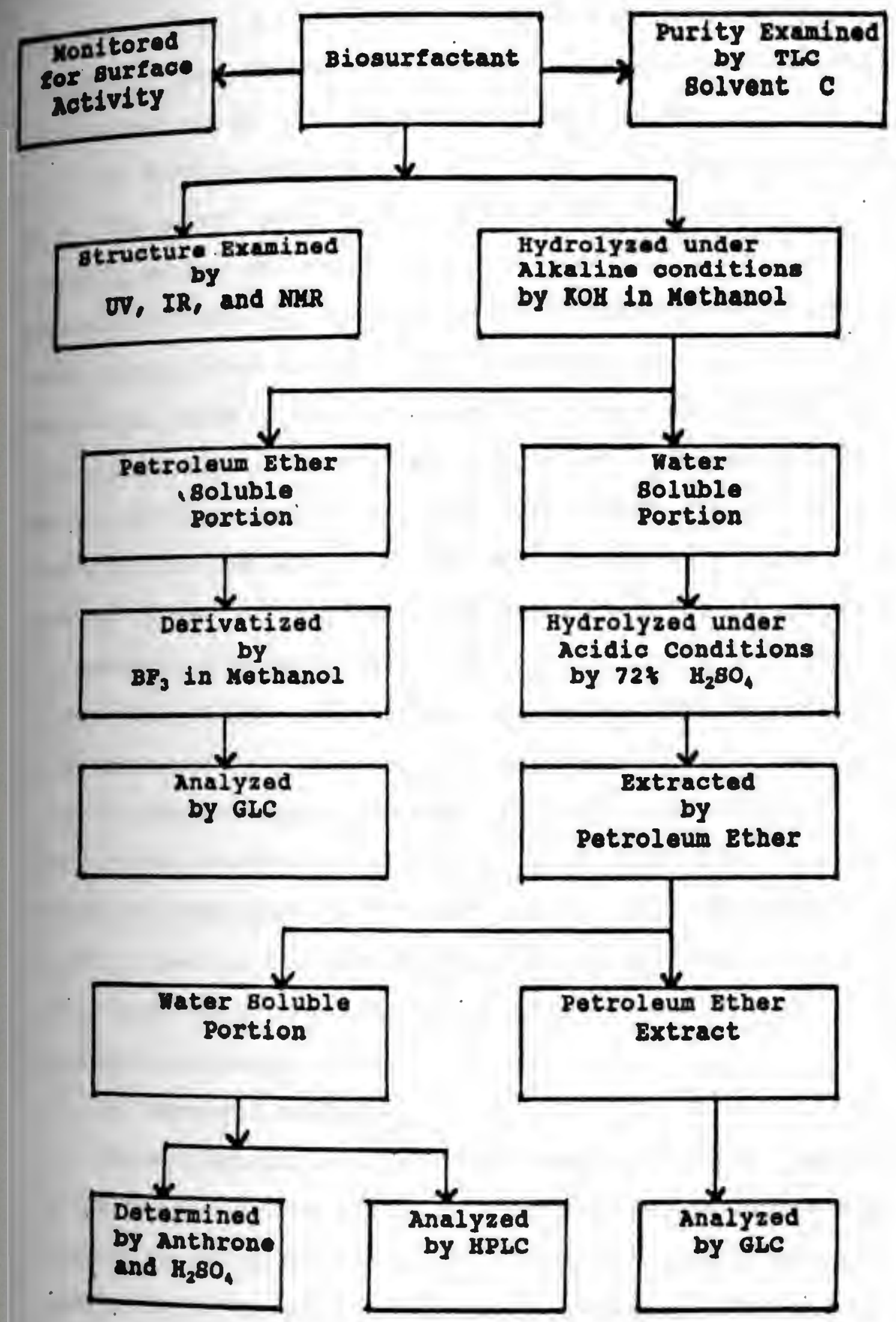


crcluding $\mathrm{C}_{10}, \mathrm{C}_{12}, \mathrm{GLC}-10,20,40,50,60,90$, and PUFA-1 (Supelco, Inc. Bellafonte, PA) were used as standards for cetermination of the relative retention times.

\section{Acid hydrolysis:}

The water phase (water soluble portion), after saponification and extraction with petroleum ether, were Aydrolyzed under acidic conditions by using $72 \%$ sulfuric acid $\mathrm{H}_{2} \mathrm{SO}_{4}$ (Adam's, 1965), then neutralized with $1 \mathrm{~N}$ sodium aydroxide $(\mathrm{NaOH})$, and extracted with petroleum ether. The series $1700^{\prime}$ gas chromatograph equipped with flame ionization detector and $10 \% \mathrm{sp} .2330$ column. The water soluble portion after acid hydrolysis was examined for the presence of reducing sugars by the anthrone-sulfuric acid method (Dreywood, 1946), and the sugars were analyzed by (HPLC) high pressure liquid chromatography (Perkin-Elmer series 10 liquid chromatography, Norwalk, CT) equipped with a refractive index detector and hydrogen sulfonated divinyl benzen styrene copolymer column, with $0.05 \mathrm{~N}$ sulfuric acid $\left(\mathrm{H}_{2} \mathrm{SO}_{4}\right)$ used as the mobile phase. 20 sugars and uronic acids were used as standards in determination of the relative retention times.

B. Physical methods:

Biosurfactant structure was examined for the presence of conjugated double bonds by scanning between wavelengths 190-900 nm using UV/VIS spectrophotometer (Perkin-Elmer, Lambda 4B UV/VIS spectrophotometer, Norwalk, CT), followed 
by analysis for determination of the functional groups using infrared spectrophotometer (Perkin-Elmer 281B Infra-red trophotometer, Norwalk, CT).

$1_{\mathrm{H}}$ and ${ }^{13} \mathrm{C}$ nuclear magnetic resonance (NMR) spectra were measured at room temperature on a Bruker AM 300 NMR pectrometer (Billerica, MA) at $300 \mathrm{MH} 2$ respectively. 


\section{CHAPTER III}

\section{RESULTS AND DISCUSSION}

Isolation, growth, and identification of the foroorganisms.

\section{Isolation of the microorganism:}

since most of the known microbial surface active agents originate from bacteria growing on hydrocarbons or related substrates, attempts have been made to screen for an organism which can assemble biosurfactant from cheap, renewable, and soluble substrates such as carbohydrates. Exposure of five plates containing nutrient agar to air for $15 \mathrm{~min}$. at different locations outside Woodward Hall, University of Rhode Island in summer of 1986 and incubation at $30^{\circ} \mathrm{C}$ for 2 days resulted in development of nine different colonies. All of the nine isolated microorganisms were orphologically characterized by utilizing gram stain reaction, four of the isolated microorganisms were found to be gram negative and the other five were gram positive bacteria.

The isolated gram positive microorganisms were grown perobically in basal mineral salts medium (BMSM) containing $200 \mathrm{mg} / 100 \mathrm{ml}$ glucose as a carbon source, then tested for surface activity. Of the five different isolated gram positive microorganisms, one was found to reduce the surface tension of the media from 74 dynes/cm to 31.4 dynes/cm as shown in table 3 (Fig. 12). The control is water which has 
Table 3. Average surface tension of the whole culture broth of the isolated gram positive organisms grown in batch culture on filter sterilized BMSM containing 0.28 glucose as C-source at $200 \mathrm{rpm}$ and $30^{\circ} \mathrm{C}$.

Fig. 12. Surface tension profile of the whole culture broth of the isolated gram positive organisms grown in batch culture on filter sterilized BMSM containing $0.2 \%$ glucose as C-source at $200 \mathrm{rpm}$ and $30^{\circ} \mathrm{C}$. 


\begin{tabular}{|c|c|c|}
\hline $\operatorname{lon}$ & $\begin{array}{l}\text { Carbon subst. } \\
(200 \mathrm{mg} / 100 \mathrm{ml})\end{array}$ & $\begin{array}{c}\text { Average 8urlece renelon } \\
\text { (Drese/an) }\end{array}$ \\
\hline 2 & Glucose & 42.7 \\
\hline 3 & Glucose & 59.6 \\
\hline 6 & Glucose & 64.2 \\
\hline 8 & Glucose & 40.5 \\
\hline 9 & Glucose & 31.4 \\
\hline
\end{tabular}

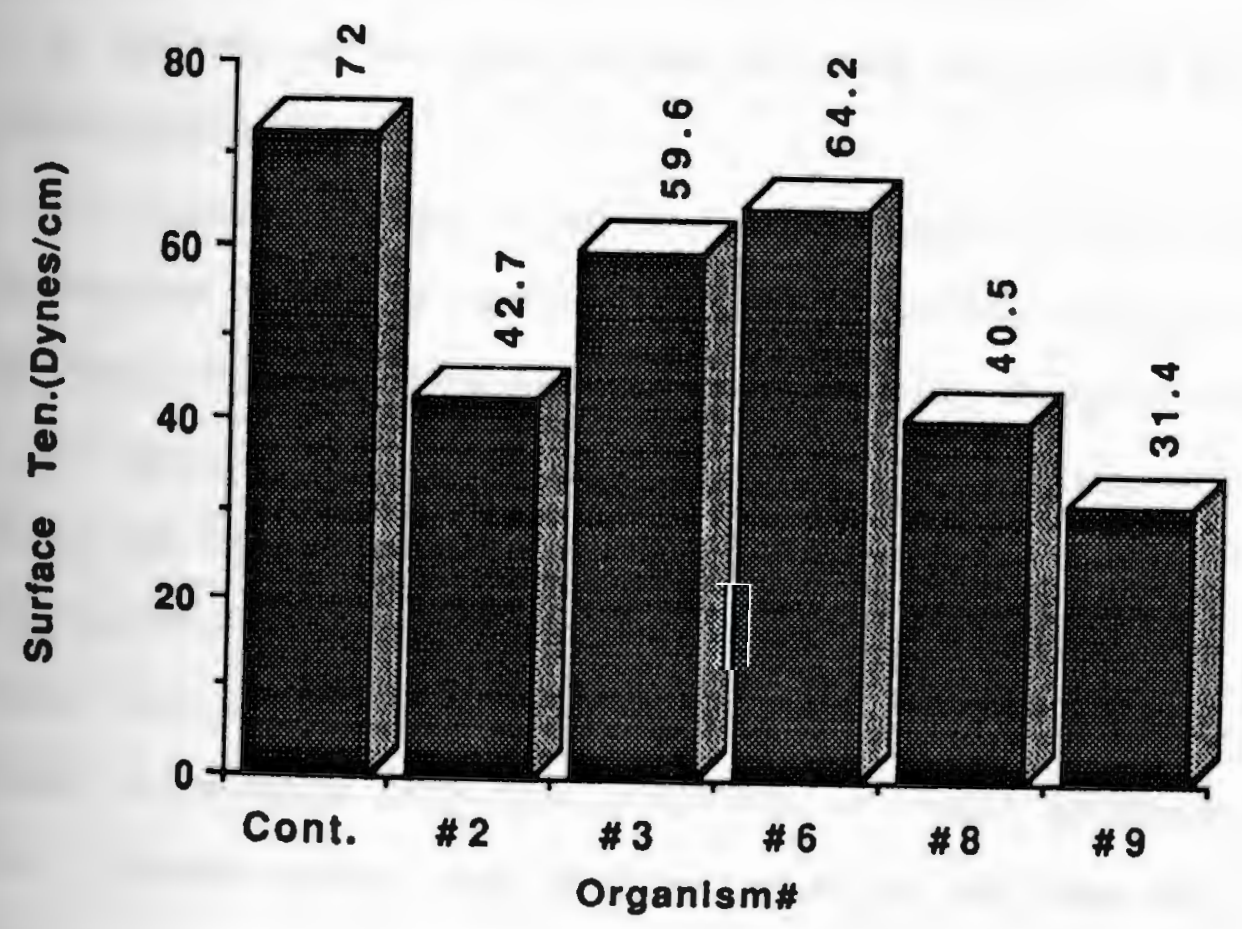


a surface tension of 72.0 dynes/cm. The five gram positive xoorganisms were also grown in BMSM containing $200 \mathrm{mg} / 100$ $\mathrm{ml}$ glucose and $20 \mathrm{mg} / 100 \mathrm{ml}$ yeast extract as growth cofactor, then tested for surface activity. The proorganism which reduced the surface tension of the media to 31.4 dynes/cm without yeast extract (table 3), was found to reduce the surface tension of the media to 30.4 dynes $/ \mathrm{cm}$ when yeast extract was added to the media as shown in table 4 (Fig. 13). Therefore the microorganism which reduced the surface tension of the culture broth the most was used as the subject for this study and was designated JIZAN-1. The Acroorganism JIZAN-1 was maintained on slants of nutrient agar at $4^{\circ} \mathrm{C}$ for regular usage, and the permanent stock culture was maintained at $-70^{\circ} \mathrm{C}$ in $2 \%$ glycerol.

2. Effect of various carbon sources on growth and yield of biosurfactant:

The microorganism JIZAN-1 was cultured in basal mineral salts medium with the water soluble substrate glucose, the water insoluble substrates sweet crude oil, Sweden crude oil, \#2 heating oil, Kuwait crude oil, and a combination of glucose and hexadecane. Good growth was observed on the media containing glucose or glucose and hexadecane as carbon sources with or without yeast extract, but growth was noticed to be more with the addition of yeast extract to the media. Slight growth was noticed when \#2 heating oil and Kuwait crude oil were used as carbon sources, but there was no growth observed when sweet or Sweden crude oils were used 
Table 4. Average surface tension obtained from the isolated gram positive organisms grown in batch culture on filter sterilized BMSM containing $0.2 \%$ glucose as C-source and $0.02 \%$ yeast extract as growth cofactor at $200 \mathrm{rpm}$ and $30^{\circ} \mathrm{C}$.

Fig. 13 Effect of yeast extract on biosurfactant production by the isolated gram positive organisms grown in batch culture on filter sterilized BMSM containing $0.2 \%$ glucose and $0.02 \%$ yeast extract at $200 \mathrm{rpm}$ and $30^{\circ} \mathrm{C}$. 


\begin{tabular}{|c|c|c|c|}
\hline Organism & $\begin{array}{l}\text { Carbon Bubst. } \\
(200 \mathrm{mg} / 100 \mathrm{ml})\end{array}$ & $\begin{array}{l}\text { Yeast Ext. } \\
\text { (20mg/100mi) }\end{array}$ & $\begin{array}{l}\text { Avg. 8uxzace } \\
\text { ronsion } \\
\text { (Dynes/Cm) }\end{array}$ \\
\hline 2 & Glucose & + & 56.6 \\
\hline 3 & Glucose & + & 63.0 \\
\hline 6 & Glucose & + & 66.9 \\
\hline 8 & Glucose & + & 35.0 \\
\hline - & Glucose & + & 30.4 \\
\hline
\end{tabular}

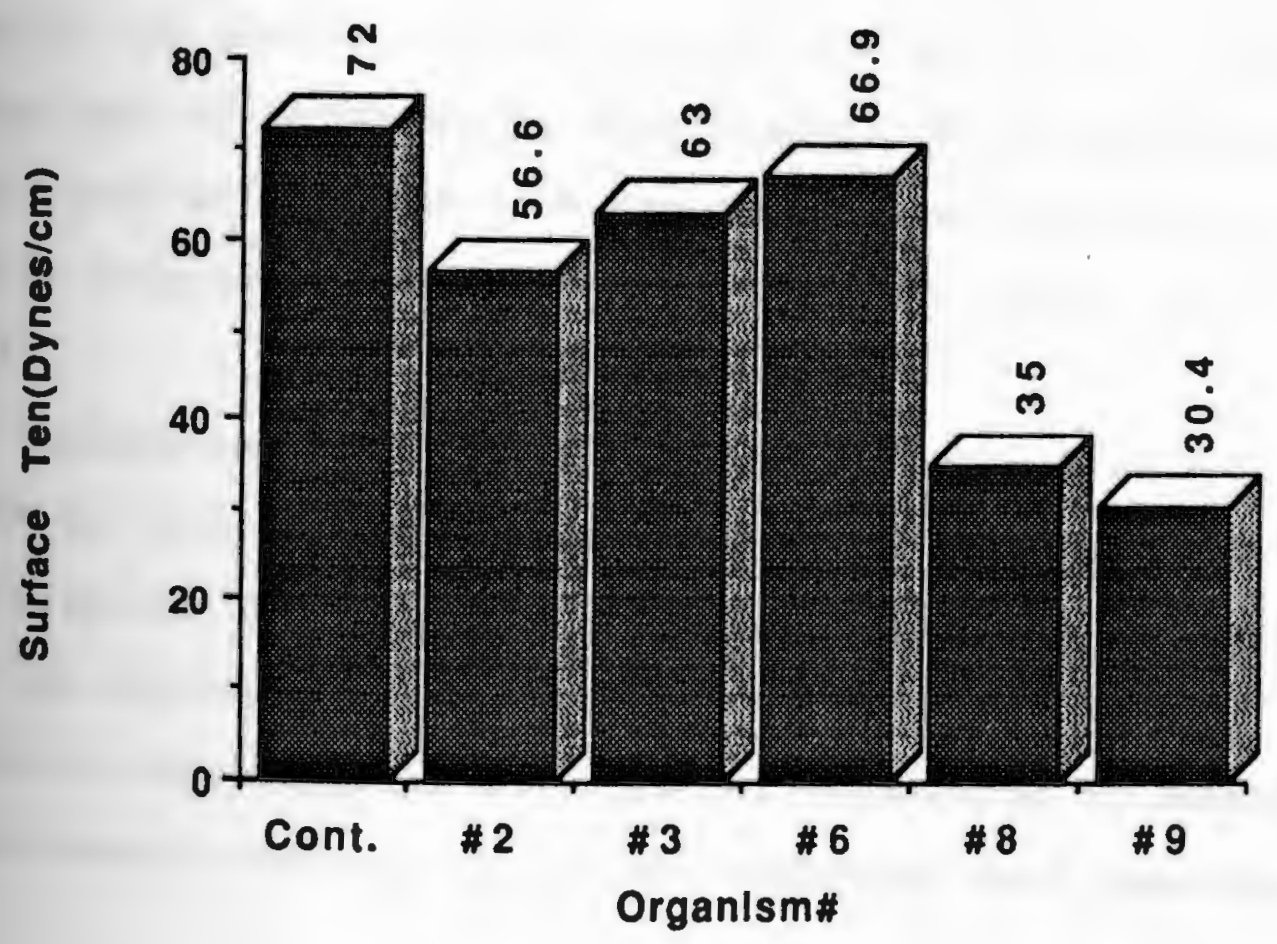


or without yeast extract caused a high reduction in the surface tension of the culture broth (i.e. production of arfactant), there was no substantial reduction in the surface tension observed when water insoluble substrates were used as carbon sources (i.e. there was no production of wurfactant during growth on water insoluble substrates). Furthermore, the addition of hexadecane to the media pontaining glucose, resulted in inhibition of biosurfactant formation as shown by table 5 (Fig. 14). A similar effect was observed in the case of surfactin production. Cooper et a1 (1981) found that the lipopeptide surfactin was produced by Bacillus subtilis from glucose, but the surfactin production was completely inhibited when hydrocarbon was added to the glucose media. An opposite effect was observed in the case of Arthrobacter paraffincus. No surface-active agent could be isolated from the medium when glucose was used as C-source instead of hexadecane (Duvnjak et al., 1982).

Glucose was found to be the best carbon source for growth and biosurfactant production by JIZAN-1, and the biosurfactant was found to be extracellular i.e. excreted into the medium by the microorganism. The influence of different physiological and physical parameters on growth and metabolic activity of the microorganism were examined. 
Table. 5 Average surface tension of the whole culture broth of Arthrobacter JIZAN-1 grown in batch culture on filter sterilized BMSM containing water-soluble (glucose) substrate and various water-insoluble substrates as C-sources with and without yeast extract at $200 \mathrm{rpm}$ and $30^{\circ} \mathrm{C}$.

Fig. 14. Effect of water-soluble (glucose) substrate and various water-insoluble substrates as C-sources on biosurfactant production by JIZAN-1 grew in batch culture on BMSM at $200 \mathrm{rpm}$ and $30^{\circ} \mathrm{C}$. 
Avg. Burf. Ten. $(20 \mathrm{mg} / 100 \mathrm{ml})$ (Dynes/On)

1 Glucose

2 Glucose

3 Glucose + Hexadecane

4 Glucose + Hexadecane

5 sweet Crude Oil

6 sweeden Crude Oil

7 12 Heating Oil

8 Kuwait crude $0 i l$

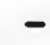

$-$

$+$

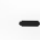

$+$

$+$

$+$

$+$

$+$
31.4

30.4

52.3

33.5

67.0

63.5

55.7

60.4

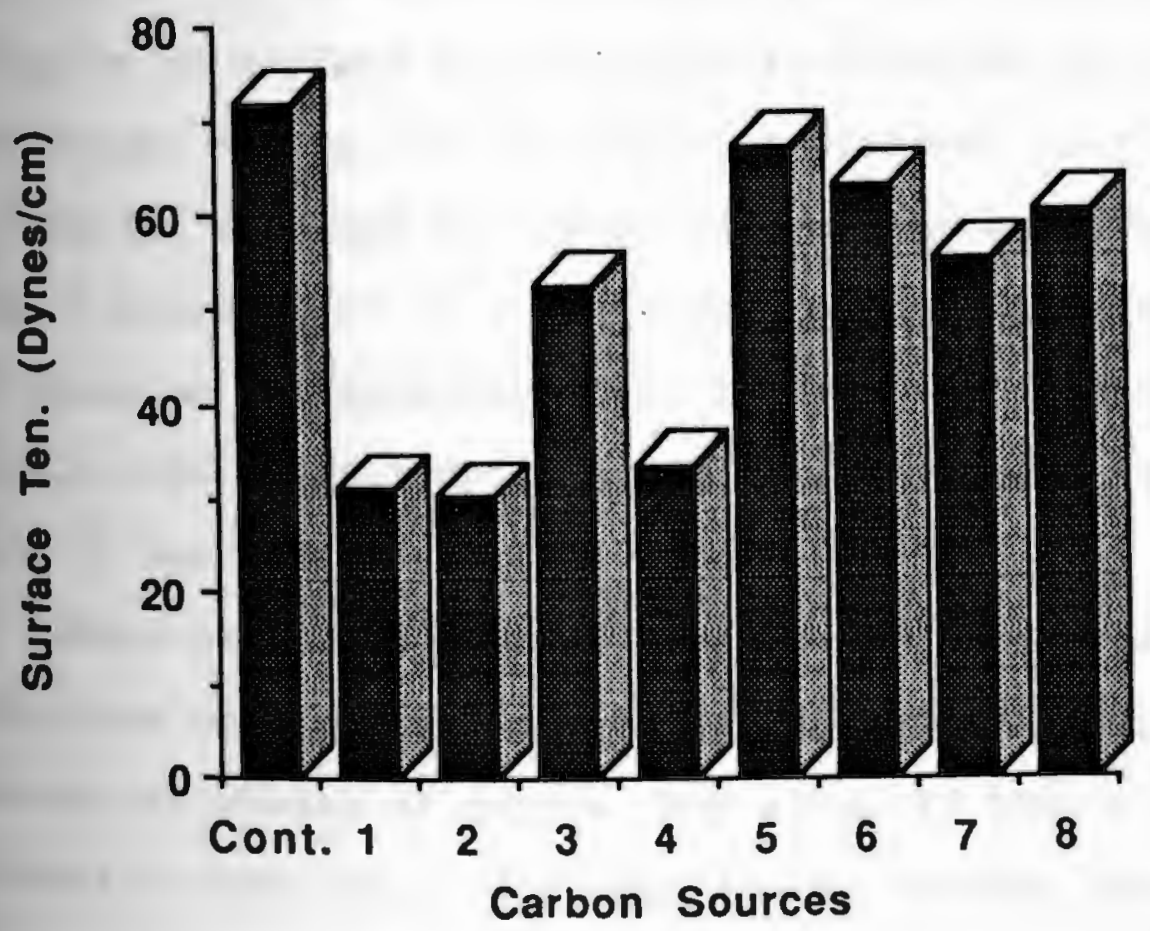


3. The relationship between growth and biosurfactant roduction:

To establish the relationship between growth response and surfactant production, JIZAN-1 was grown in BMSM containing 0.28 glucose, incubated at $200 \mathrm{rpm}$ and $30^{\circ} \mathrm{C}$ for 36 hrs. Growth, surface and interfacial tensions were measured at intervals during the $36 \mathrm{hr}$. experiment.

Growth was monitored by measuring the turbidity using Klett-Summerson photoelectric colorimeter (blue filter), and biosurfactant production was monitored during growth by measuring the surface tension and the interfacial tension against \#2 heating oil using a Fisher autotensiomat. (Fig. 15).

A plot of turbidity with surface and interfacial tensions versus incubation period (Fig. $16 \mathrm{a}, \mathrm{b})$ shows the variation of surface and interfacial tensions (biosurfactant production) during the fermentation process. Surface and Interfacial tensions of the culture medium quickly dropped from 73 dynes/cm to 27.3 dynes/cm and from 18 dynes/cm to 2.5 dynes/cm respectively after $15 \mathrm{hrs}$. of incubation, then rapidly rose to 56.3 dynes/cm and 11.5 dynes/cm respectively after $36 \mathrm{hrs.}$ of incubation.

These data indicated that maximum biosurfactant production occurred during the lag phase and early exponential phases of growth, but after 15 hrs. of Incubation there was a decrease in the surface tension and aterfacial tension of the culture supernatant as shown by 
Fig. 15. Biosurfactant production was monitored during or after cultivation by measuring the surface tension of the culture broth and the interfacial tension of the culture broth against \#2 heating oil using Fisher autotensiomat.

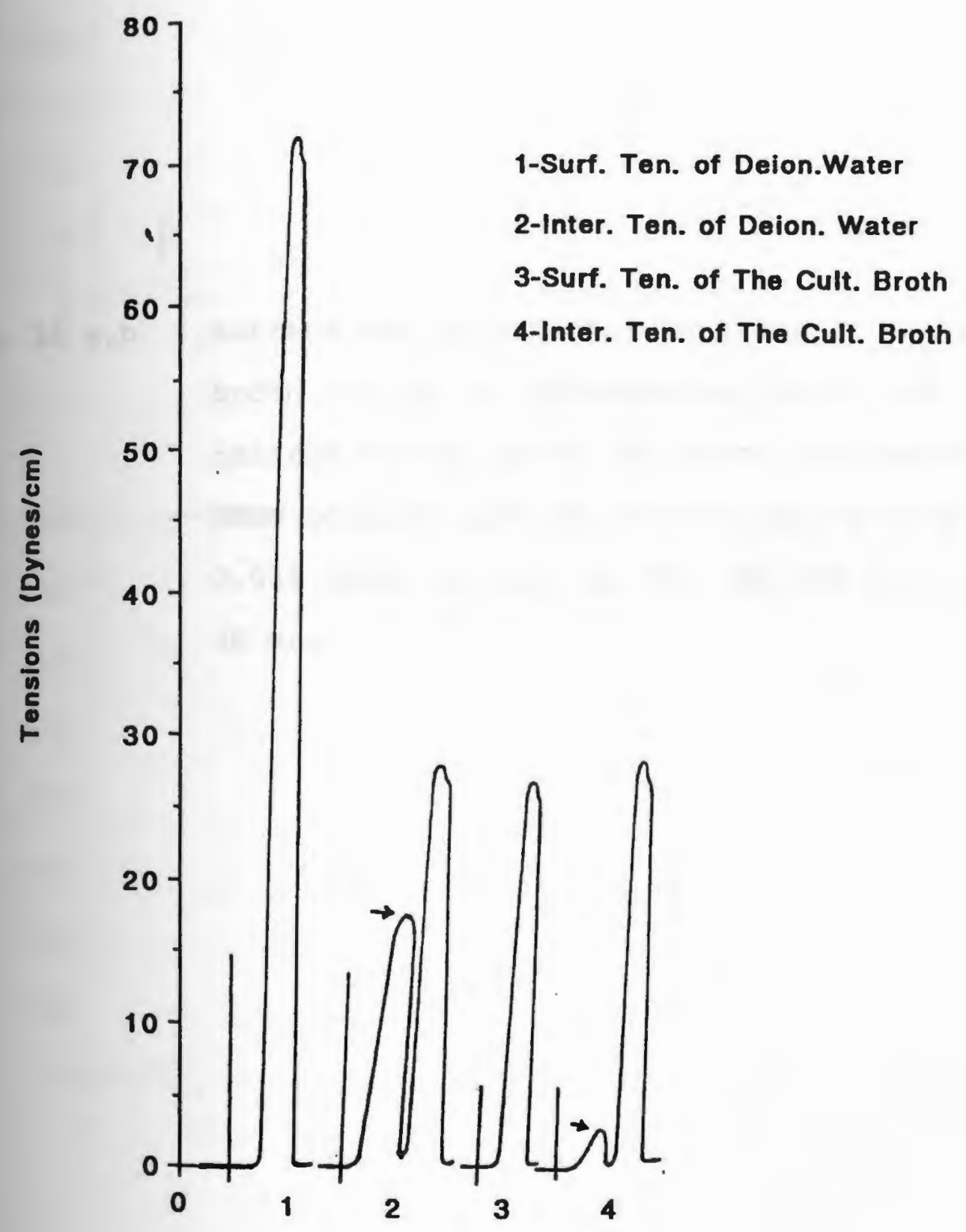


Fig. $16 a, b$ Surface and interfacial tensions of the whole broth culture of Athrobacter JIZAN-1 as related to its growth in batch culture on BMSM containing $0.2 \%$ glucose as C-source and $0.02 \%$ yeast extract at $200 \mathrm{rpm}$ and $30^{\circ} \mathrm{C}$ for 36 hrs. 

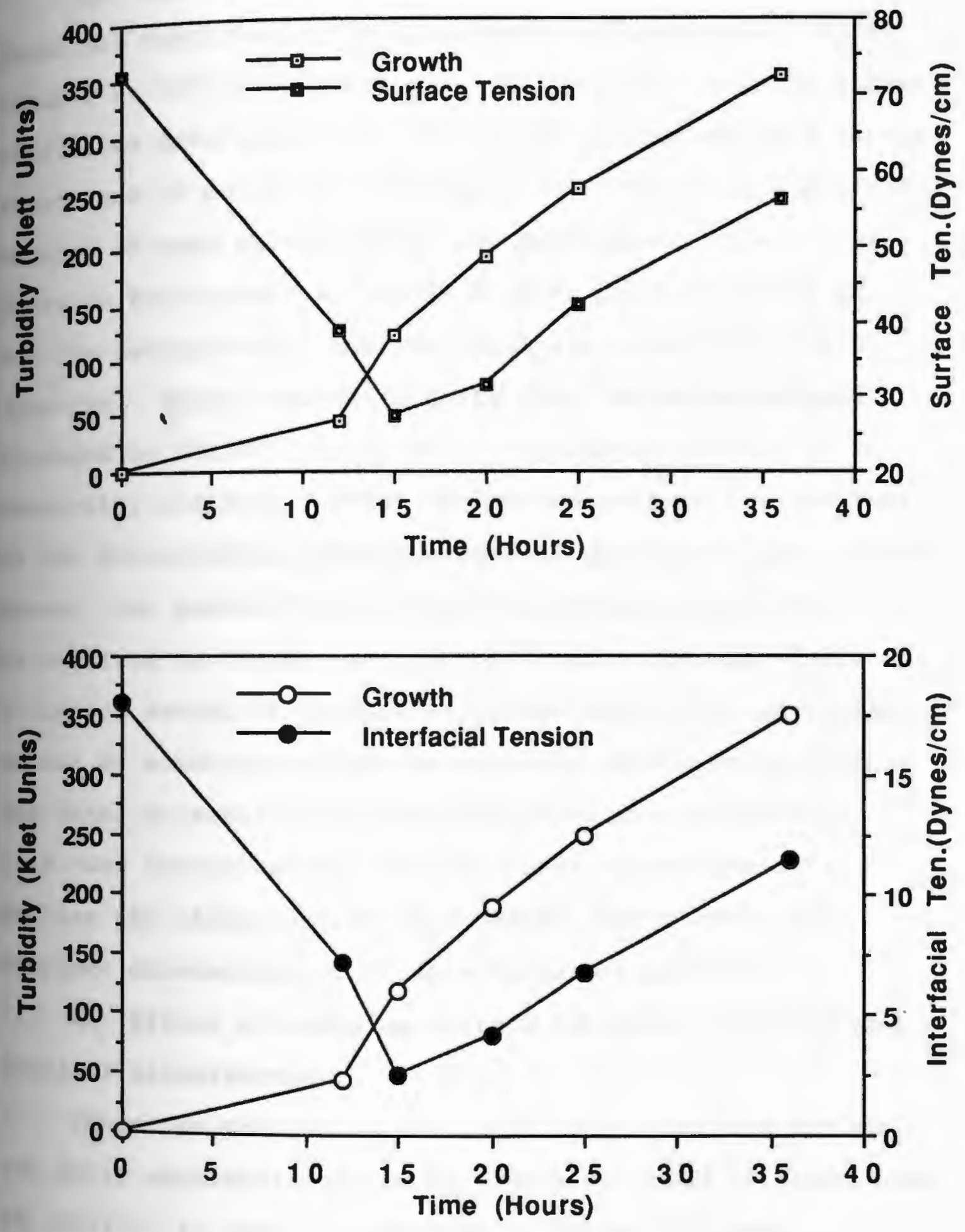
Fig. 16 a,b.

The loss of biosurfactant activity in the late growth phase was confirmed by incubation of duplicate cultures of JIZAN- 1 at $30^{\circ} \mathrm{C}$ with shaking at $200 \mathrm{rpm}$ for 4 days on filter terilized BMSM containing $200 \mathrm{mg} / 100 \mathrm{ml}$ glucose as a carbon source and $20 \mathrm{mg} / 100 \mathrm{ml}$ yeast extract. The average surface tension of each culture broth was determined, it was 60.2 dynes/cm for culture \#1 and 55.8 dynes/cm for culture \#2, and the average value for the duplicate culture was 58.0 dynes/cm. These results indicate that the biosurfactant produced by JIZAN-1 is either biodegradable or that it is themically modified. There may be two reasons for the loss of the biosurfactant from the culture during the late growth phase. One possibility is that the biosurfactant was Fe-utilized by JIZAN-1 as a carbon source, because there was a limited amount of glucose as carbon source and an excess amount of ammonium nitrate as nitrogen source in the media. The other possibility is that the inoculum was not pure (i.e. was contaminated), and the other microorganism(s) utilize the biosurfactant as a carbon source after the complete consumption of glucose as carbon source.

4. Effect of ammonium nitrate $\left(\mathrm{NH}_{4} \mathrm{NO}_{3}\right)$ on growth and rield of biosurfactant:

Since the production of biosurfactant occured during the early exponential phase of growth followed by rapid loss of activity in the late exponential phase of growth, ammonium nitrate as a $\mathrm{N}$-source was tested at different 
ancentrations to determine if there was a relation between the loss of biosurfactant activity and the concentration of $\mathrm{NH}_{4} \mathrm{NO}_{3}$. Ammonium nitrate was tested at $50 \mathrm{mg}$ increment over the range of $50-250 \mathrm{mg} / 100 \mathrm{ml}$. Fig. $17 \mathrm{a}, \mathrm{b}$ shows the effect of $\mathrm{NH}_{4} \mathrm{NO}_{3}$ concentration on growth and yield of biosurfactant in a filter sterilized BMSM containing $200 \mathrm{mg} / 100 \mathrm{ml}$ glucose as a carbon source and $20 \mathrm{mg} / 100 \mathrm{ml}$ yeast extract. Maximum yield (biomass concentration) was obtained with 50 to 150 $\mathrm{mg} / 100 \mathrm{ml}$ of ammonium nitrate after $12 \mathrm{hrs}$. of incubation. The biosurfactant activity was maximum at $100-150 \mathrm{mg} / 100 \mathrm{ml}$ of $\mathrm{NH}_{4} \mathrm{NO}_{3}$ after $12 \mathrm{hrs}$. of incubation. At $\mathrm{NH}_{4} \mathrm{NO}_{3}$ soncentration above $150 \mathrm{mg} / 100 \mathrm{ml}$ there was inhibition of growth and a corresponding decrease in biosurfactant activity. Thus ammonium nitrate concentration as $\mathrm{N}$-source was found to play an important role in the regulation of blosurfactant synthesized by JIZAN-1.

A similar effect was observed with growing cells of Pseudomonas sp. DSM 2874, when rhamnolipid production was increased after $\mathrm{N}$-limitation using sodium nitrate or anmonium sulfate as nitrogen sources (Guerr et al., 1984). An opposite effect was observed with different Corynebacteria, while nitrate as the sole $\mathrm{N}$-source resulted in a fast increase of surfactant concentration during mid-exponential growth phase, ammonium plus nitrate resulted in growth-associated surfactant production (Gerson and Zajic, 1979). 
Fig. $17 \mathrm{a}, \mathrm{b}$ Surface and interfacial tensions of the whole culture broth of JIZAN-1 as related to its growth in batch culture on BMSM containing various amount of $\mathrm{NH}_{4} \mathrm{NO}_{3}$ (50-250 with 50 $\mathrm{mg} / 100 \mathrm{ml}$ increment) as $\mathrm{N}$-source, $0.2 \%$ glucose as C-source and $0.02 \%$ yeast extract at $200 \mathrm{rpm}$ and $30^{\circ} \mathrm{C}$ for $12 \mathrm{hrs}$. 

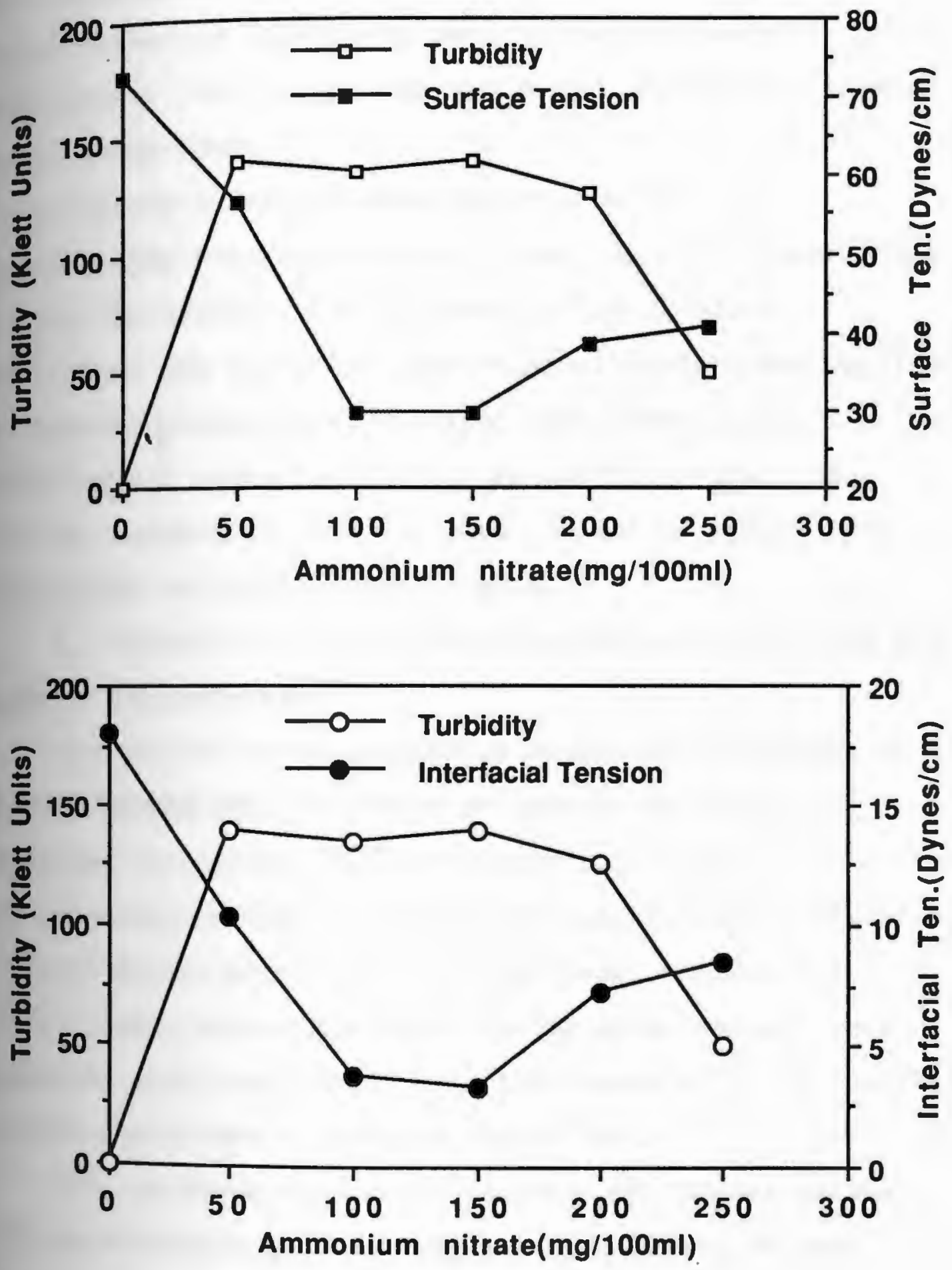
since the maximum growth and biosurfactant production were obtained with $150 \mathrm{mg} / 100 \mathrm{ml}$ of $\mathrm{NH}_{4} \mathrm{NO}_{3}$, this encentration of $\mathrm{NH}_{4} \mathrm{NO}_{3}$ was used in the following experiments. This media was designated as modified mineral salts medium (MMSM).

A second possible reason behind the loss of blosurfactant from the culture during the late growth phase is that the original culture used in the previous uperiments was not pure. The original isolate was purified by repeated,streaking on nutrient agar under strict sterilization conditions to ensure uniform colony and cellular morphology, and the pure isolate of JIZAN-1 was eransferred to nutrient agar slants.

5. Effect of glucose concentration on growth rate and yield of biosurfactant:

Glucose as carbon and energy source at increments of 50 $\mathrm{mg}$ were tested over the range of $100-400 \mathrm{mg} / 100 \mathrm{ml}$ to determine the optimal glucose concentration for Mcroorganism (JIZAN-1) growth and biosurfactant production. A $100 \mathrm{ml}$ filter sterilized modified basal mineral salts medium (MMSM) containing $20 \mathrm{mg} / 100 \mathrm{ml}$ yeast extract were placed in side arm flasks, then inoculated with the purified isolate and shaken at $200 \mathrm{rpm}$ and at $30^{\circ} \mathrm{C}$.

Fig. 18 shows the growth response of JIZAN-1 versus time at different glucose concentrations up to $18 \mathrm{hrs}$. incubation. The highest growth rate was observed with 
lucose at $200 \mathrm{mg} / 100 \mathrm{ml}$, and the lowest growth rate was at $100 \mathrm{mg} / 100 \mathrm{ml}$. The differences in growth rate were minor but the effect on the phase of decline and maximum cell yield are more apparent as shown by Fig. 18 .

Fig. 19 shows that, there was no major differences between the surface tension values obtained after $18 \mathrm{hrs}$. of growth on all glucose concentration tested. Growth of the isolated microorganism (JIZAN-1) and the subsequent Froduction of biosurfactant were found to be dependent on a soluble substrate (glucose) rather than crude oils and pure Marocarbon. The concentration of glucose had a slight effect on the microorganism growth, but did not influence the biosurfactant production.

6. Effect of the incubation temperature on growth of the microorganisms:

The effect of temperature over the range of $20-35^{\circ} \mathrm{C}$ (at $5^{\circ} \mathrm{C}$ increment) was studied to determine the optimum temperature for growth. Filter sterilized MMSM containing $200 \mathrm{mg} / 100 \mathrm{ml}$ glucose as a carbon source and $20 \mathrm{mg} / 100 \mathrm{ml}$ yeast extract was inoculated with $1.0 \mathrm{ml}$ of a $12 \mathrm{hrs}$. old culture. Fig. 20 a, b shows the effect of various incubation temperature on growth of the microorganism during a 20 hrs. cultivation period. The highest growth (biomass concentration) was obtained at $30^{\circ} \mathrm{C}$, but a decrease in maximum biomass concentration was occurred at $35^{\circ} \mathrm{C}$ and $25^{\circ} \mathrm{C}$. Lower growth rates, increased lag time and lower final cell mass were obtained at all temperature tested except at $30^{\circ} \mathrm{C}$. 
Fig. 18 Arthrobacter JIZAN-1 growth rate in batch culture on filter sterilized MMSM containing various amount of glucose (100-400 with $50 \mathrm{mg} / 100 \mathrm{ml}$ increment) as c-source and $0.02 \%$ yeast extract at $200 \mathrm{rpm}$ and $30^{\circ} \mathrm{C}$ for $18 \mathrm{hrs}$. 


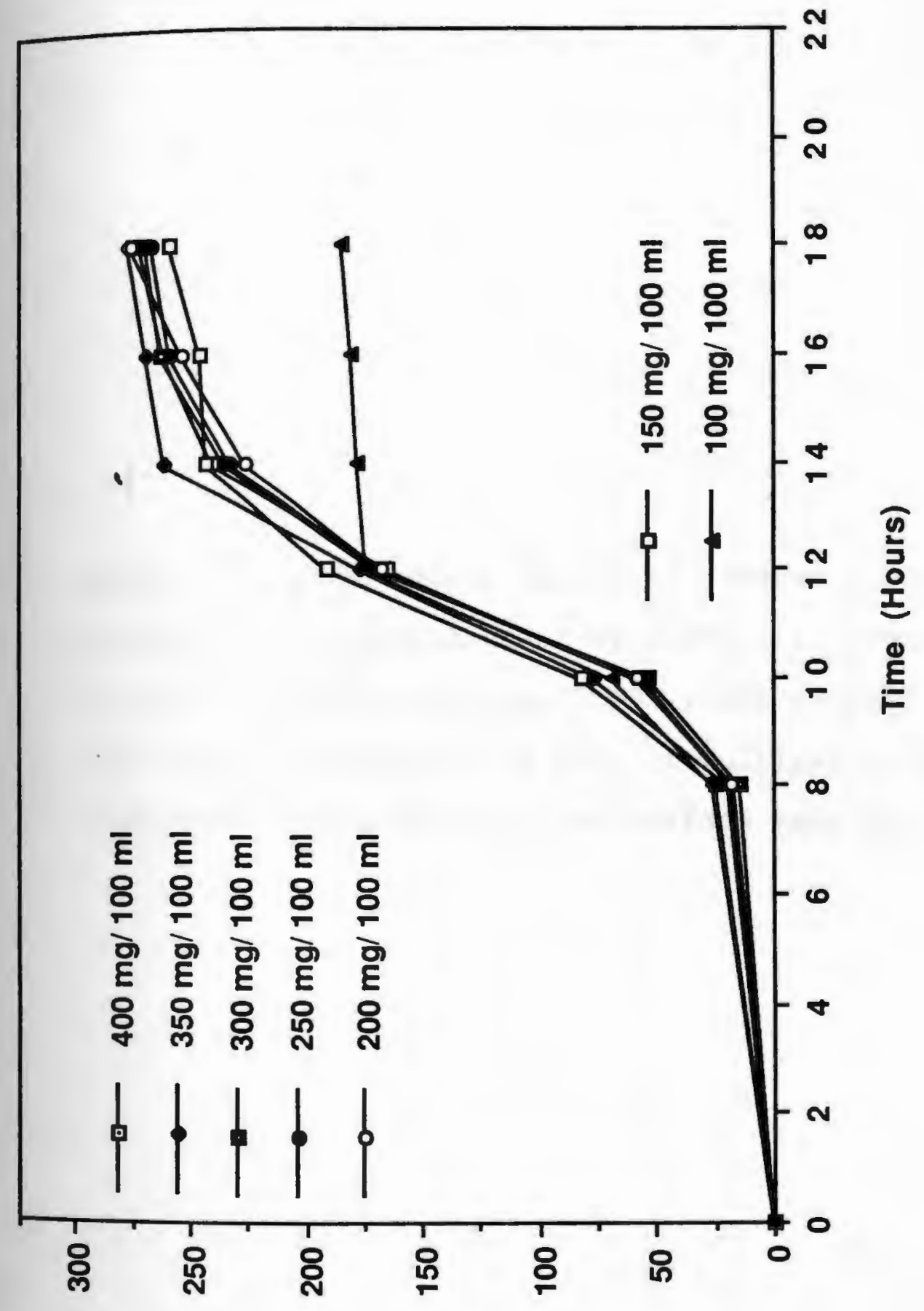

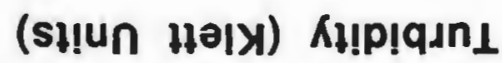


Fig. 19 Effect of carbon source (glucose) concentration on production of biosurfactant by JIZAN-1 in batch culture on MMSM containing $0.02 \%$ yeast extract at $200 \mathrm{rpm}$ and $30^{\circ} \mathrm{C}$ after $18 \mathrm{hrs}$. of cultivation as indicated by the reduction of surface tension. 


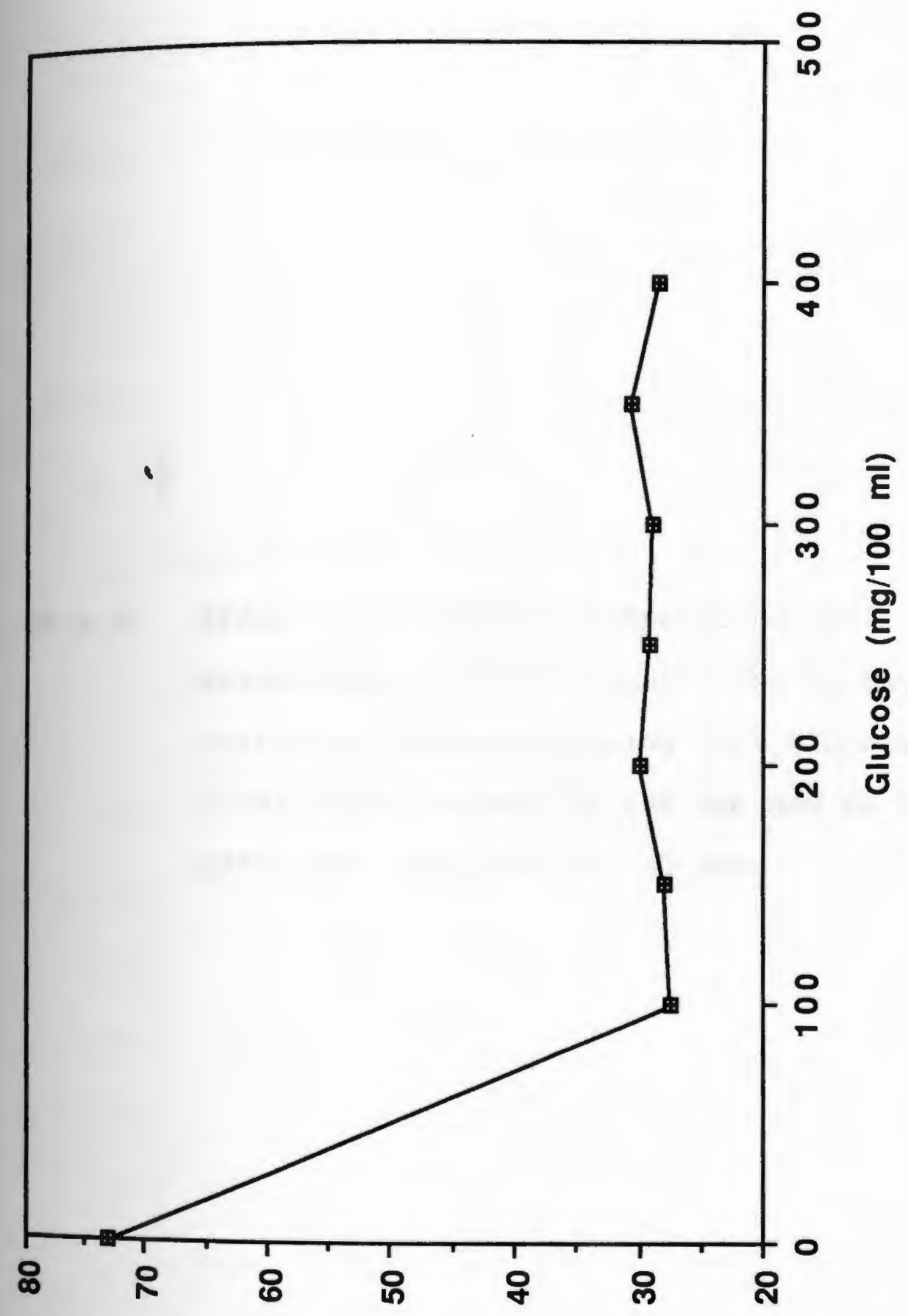

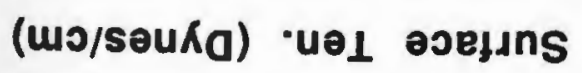


Fig. $20 \mathrm{a}, \mathrm{b}$ Effect of incubation temperature on Arthrobacter JIZAN-1 growth rate in batch culture on MMSM containing $0.2 \%$ glucose and $0.02 \%$ yeast extract at $200 \mathrm{rpm}$ and at $20^{\circ} \mathrm{C}$, $25^{\circ} \mathrm{C}, 30^{\circ} \mathrm{C}$ and $35^{\circ} \mathrm{C}$ for $20 \mathrm{hrs}$. 

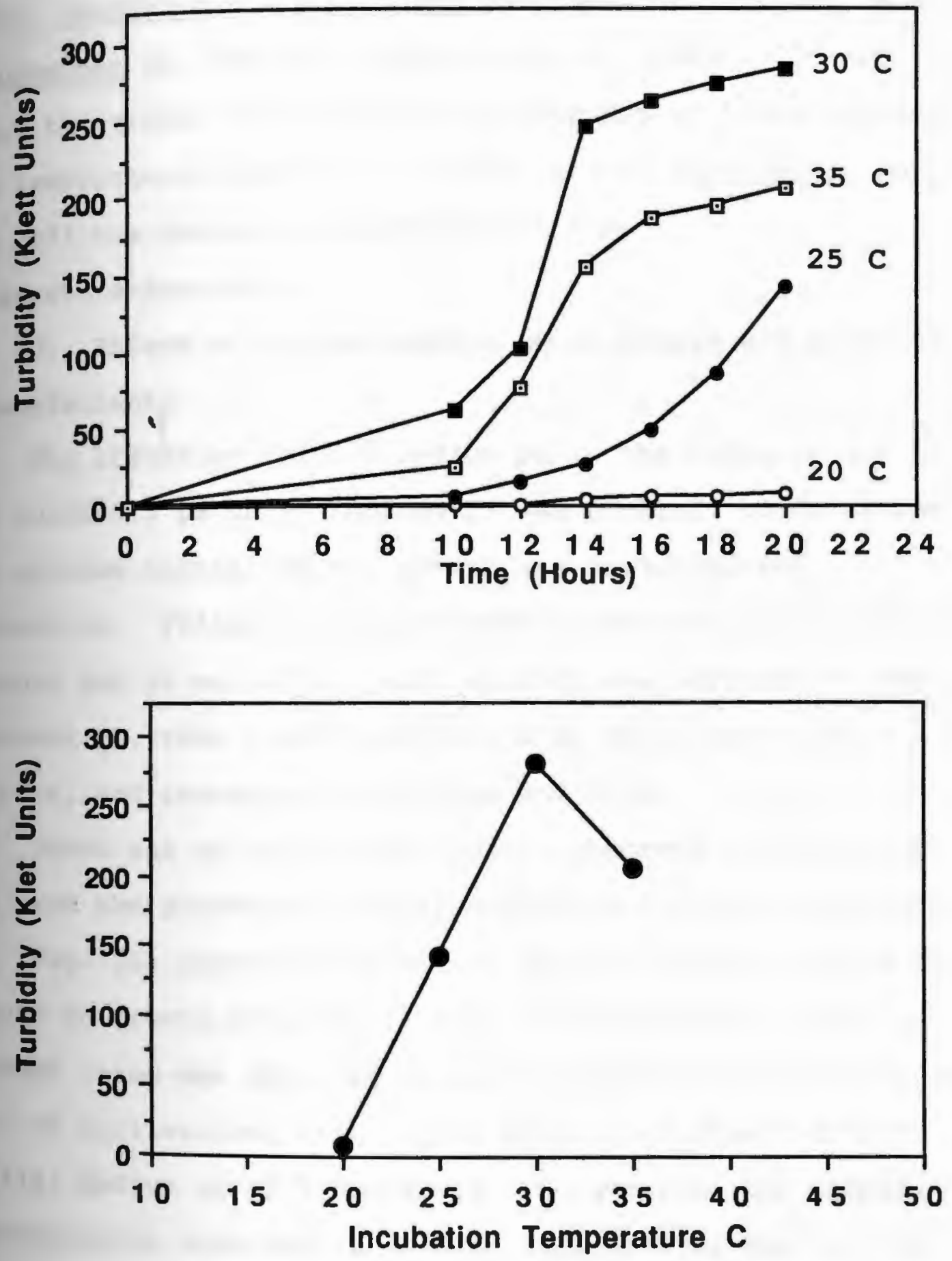
remperature was found to be of great important in the case of Nthrobacter paraffineus ATCC 19558 (Duvnjak et al., 1982), Rhodococcus erythropolis (Wagner et al., 1984), and gseudomonas sp. DSM 2874 (Syldatk et al., 1985). In all cases the growth and subsequent production of biosurfactant was temperature-dependent. In the case of Pseudomonas sp. DSM 2874 the product composition was also temperature-dependent.

7. Effect of initial medium $\mathrm{pH}$ on growth and yield of biosurfactant:

The effect of initial medium $\mathrm{pH}$ in the range of 4.0 to 7.5 (with $0.5 \mathrm{pH}$ units increment) was studied, to determine the optimum initial pH for growth and biosurfactant production. Filter sterilized MMSM containing $200 \mathrm{mg} / 100 \mathrm{ml}$ glucose and $20 \mathrm{mg} / 100 \mathrm{ml}$ yeast extract was adjusted to the intended $\mathrm{pH}$, then inoculated with $1 \mathrm{ml}$ of $12 \mathrm{hrs}$. old culture, and incubated at $200 \mathrm{rpm}$ and $30^{\circ} \mathrm{C}$.

There was no growth (turbidity) observed at $\mathrm{pH} 4.0$ or 4.5, and the growth at initial medium $\mathrm{pH} 5.0$ was negligible. Fig. $21 \mathrm{a}$ shows the effect of various initial medium $\mathrm{pH}$ values on growth over the $18 \mathrm{hrs}$. of cultivation. The highest value was obtained at initial medium $\mathrm{pH} 7.0$ after 18 hrs. of cultivation, also a good growth was observed with initial medium $\mathrm{pH}$ of 7.5 and 6.5. In general, the biomass concentration obtained at $18 \mathrm{hrs}$. decreased as the initial medium $\mathrm{pH}$ value decreased except that the growth was lightly less with $\mathrm{pH} 7.5$ than with $\mathrm{pH} 7.0$. 
Fig. 21 a Effect of initial medium $\mathrm{pH}$ (4.0-7.5 with $0.5 \mathrm{pH}$ unit increment) on Arthrobacter JIZAN-1 growth rate in batch culture on MMSM containing $0.2 \%$ glucose and $0.02 \%$ yeast extract at $200 \mathrm{rpm}$ and $30^{\circ} \mathrm{C}$ for $18 \mathrm{hrs}$.

Fig. $21 \mathrm{~b}$ Effect of initial medium $\mathrm{pH}$ (4.0-7.5 with 0.5 unit increment) on biosurfactant production by JIZAN-1 grown in batch culture on MMSM containing $0.2 \%$ glucose and $0.02 \%$ yeast extract at $200 \mathrm{rpm}$ and $30^{\circ} \mathrm{C}$ for $18 \mathrm{hrs}$, as determined by the reduction of surface and interfacial tensions. 

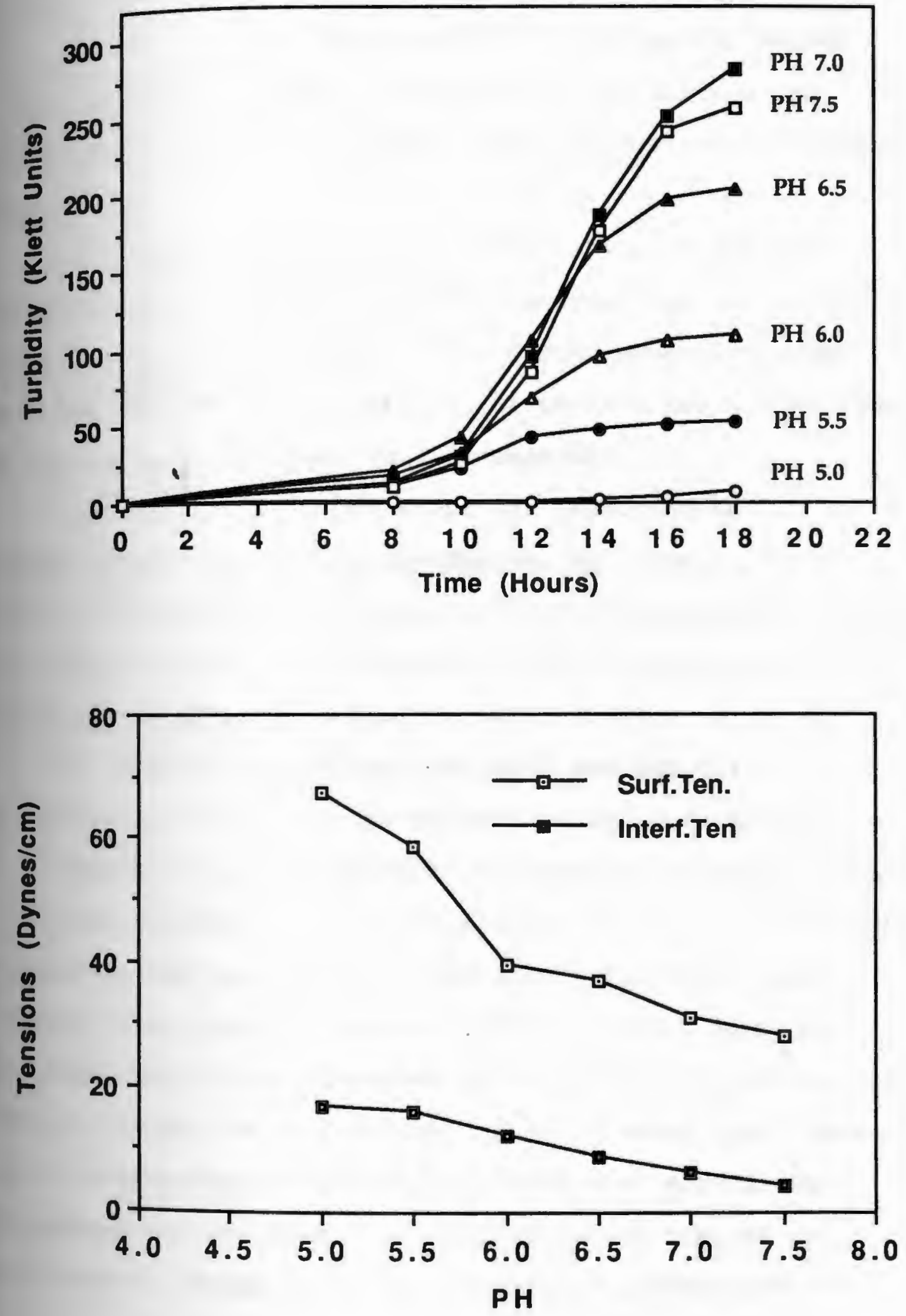
In

terms of total biomass production $\mathrm{pH} 7.0$ was optimum.

Fig. $21 \mathrm{~b}$ shows the effect of initial medium $\mathrm{pH}$ on rurfactant production. In general, the surface and Cerfacial tensions decreases with the increases of initial medium $\mathrm{pH}$.

Kosaric et al. (1987) found that, biosurfactant Mnthesis and the growth of the microorganisms often have dfferent optimal pH values. The differences in growth response and surfactant activity at pHs 7.0 and 7.5 as shown by Fig. 21 a b confirmed this assumption.

The initial medium $\mathrm{pH}$ value was important in Mamnolipid production by Pseudomonas sp. (Wagner et al., 1984), cellobiose lipid formation by Ustilago maydis (Frantz and Wagner, 1984), and in sophorolipid formation by Torulopsis bombicola (Gobbert et al., 1984).

\section{Effect of $\mathrm{N}$-sources $\left(\mathrm{NH}_{4} \mathrm{NO}_{3}\right)$ and $\left(\mathrm{NH}_{4} \mathrm{Cl}\right)$}

concentration on growth and biosurfactant production:

Ammonium nitrate $\left(\mathrm{NH}_{4} \mathrm{NO}_{3}\right)$ and ammonium chloride $\left(\mathrm{NH}_{4} \mathrm{Cl}\right)$ at $50 \mathrm{mg}$ increments in the range of $50-350 \mathrm{mg} / 100 \mathrm{ml}$ in BMSM containing $200 \mathrm{mg} / 100 \mathrm{ml}$ glucose and $20 \mathrm{mg} / 100 \mathrm{ml}$ yeast extract, were inoculated with $1 \mathrm{ml}$ of a $12 \mathrm{hr}$. inoculum. The fermentation was incubated at $30^{\circ} \mathrm{C}$ with agitation at 200 rpm for 22 hrs. of cultivation. Fig. 22 shows that, there was no substantial different in growth with any of the concentrations of $\mathrm{NH}_{4} \mathrm{NO}_{3}$ used during the $22 \mathrm{hrs}$. of cultivation, except a slight increase of growth with 150 
$\mathrm{mg} / 100 \mathrm{mI}$ in the early stage of growth and a slight decrease in the biomass concentration with $50 \mathrm{mg} / 100 \mathrm{ml}$ in the late stage of growth.

similar effects were observed when ammonium chloride $\left(\mathrm{NH}_{4} \mathrm{CI}\right)$ was used as $\mathrm{N}$-source as shown by Fig. 23, i.e. there was no substantial difference between growth at any $\mathrm{NH}_{4} \mathrm{Cl}$ concentration used during the $20 \mathrm{hrs}$. of cultivation, except with the $350 \mathrm{mg} / 100 \mathrm{ml} \mathrm{NH} 4 \mathrm{Cl}$ concentration, the growth was less than the growth on all of the other concentrations of $\mathrm{NH}_{4} \mathrm{Cl}$ used, through all stages of growth.

The growth (biomass concentration) was higher at 10 hrs. on $\mathrm{NH}_{4} \mathrm{Cl}$ as $\mathrm{N}$-source than on $\mathrm{NH}_{4} \mathrm{NO}_{3}$, yet the final mass was much greater in the case of ammonium nitrate. Although in the standard test for nitrate reduction this organism (JIZAN-1) tested negative for nitrite these data would indicate that assimilatory nitrate reduction does occur. The increased cell mass with $\mathrm{NH}_{4} \mathrm{NO}_{3}$ indicates that the $\mathrm{NO}_{3}$ moiety is reduced to $\mathrm{NH}_{4}$ which is assimilated, rather than to $\mathrm{N}_{2}$ which allows the accumulation of $\mathrm{NO}_{2}$. The effect of $\mathrm{N}$-sources $\left(\mathrm{NH}_{4} \mathrm{NO}_{3}\right)$ and $\left(\mathrm{NH}_{4} \mathrm{Cl}\right)$ concentration on biosurfactant production were determined by monitoring the surface activity of the culture broth after the cultivation period, which was $22 \mathrm{hrs}$. in the case of $\mathrm{NH}_{4} \mathrm{NO}_{3}$ and $20 \mathrm{hrs}$. in the case of $\mathrm{NH}_{4} \mathrm{Cl}$, using Fisher autotensiomat (Fig. $24 \mathrm{a}, \mathrm{b}$ and 25). Surface and interfacial tensions decreased with increased concentration of both $N$-sources $\left(\mathrm{NH}_{4} \mathrm{NO}_{3}\right)$ and $\left(\mathrm{NH}_{4} \mathrm{Cl}\right)$, i.e. the biosurfactant 
Fig. 22 Effect of ammonium nitrate $\left(\mathrm{NH}_{4} \mathrm{NO}_{3}\right)$ concentration (50-350 with $50 \mathrm{mg} / 100 \mathrm{ml}$ increment) on Arthrobacter JIZAN-1 growth rate in batch culture on BMSM containing $0.2 \%$ glucose and $0.02 \%$ yeast extract at $200 \mathrm{rpm}$ and $30^{\circ} \mathrm{C}$ for $22 \mathrm{hrs}$. 


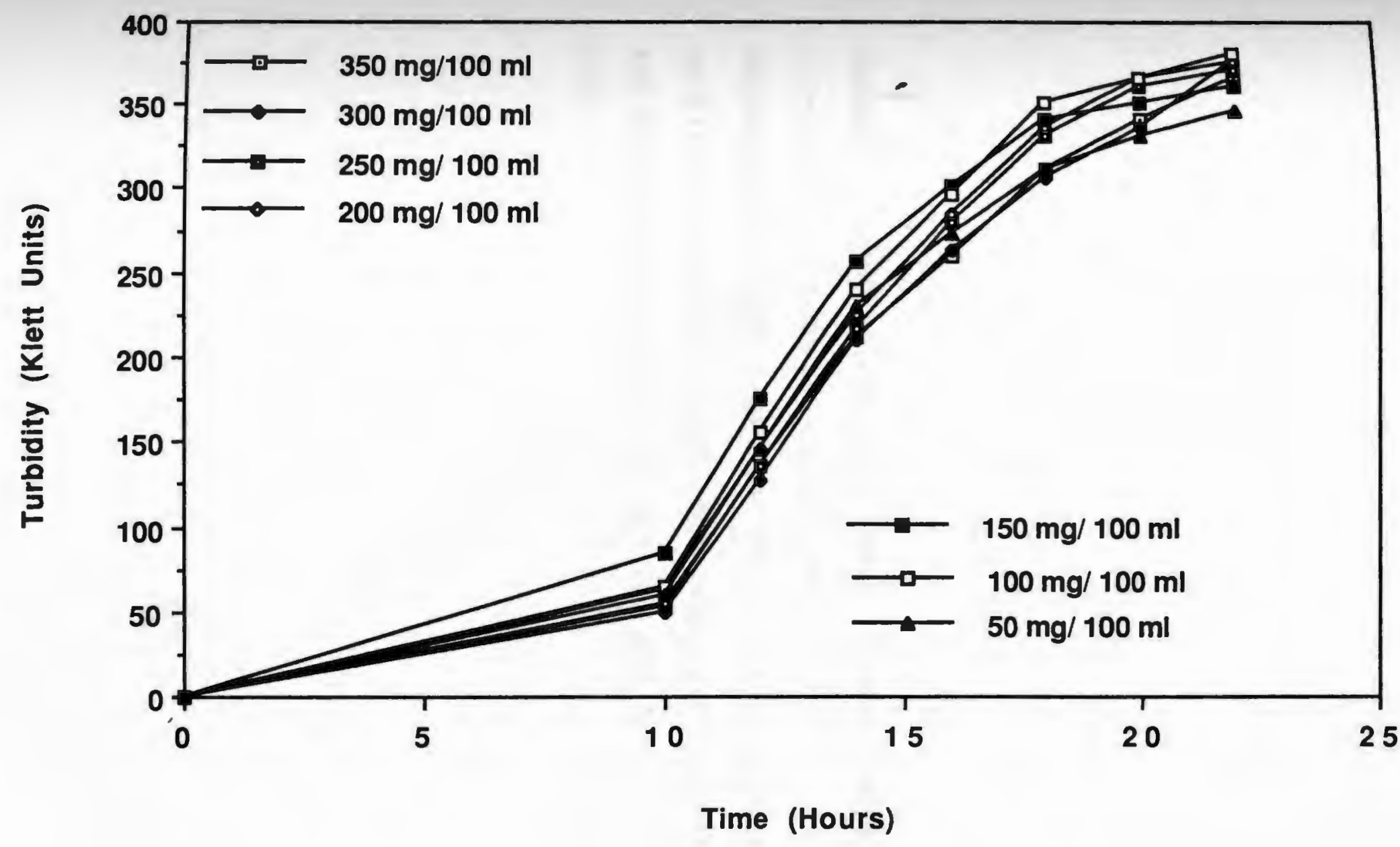


Fig. 23 Effect of ammonium chloride $\left(\mathrm{NH}_{4} \mathrm{Cl}\right)$ concentration (50-350 with $50 \mathrm{mg} / 100 \mathrm{ml}$ increment) on Arthrobacter JIZAN-1 growth rate in batch culture on filter sterilized BMSM containing $0.2 \%$ glucose and $0.02 \%$ yeast extract at $200 \mathrm{rpm}$ and $30^{\circ} \mathrm{C}$ for 20 hrs. 


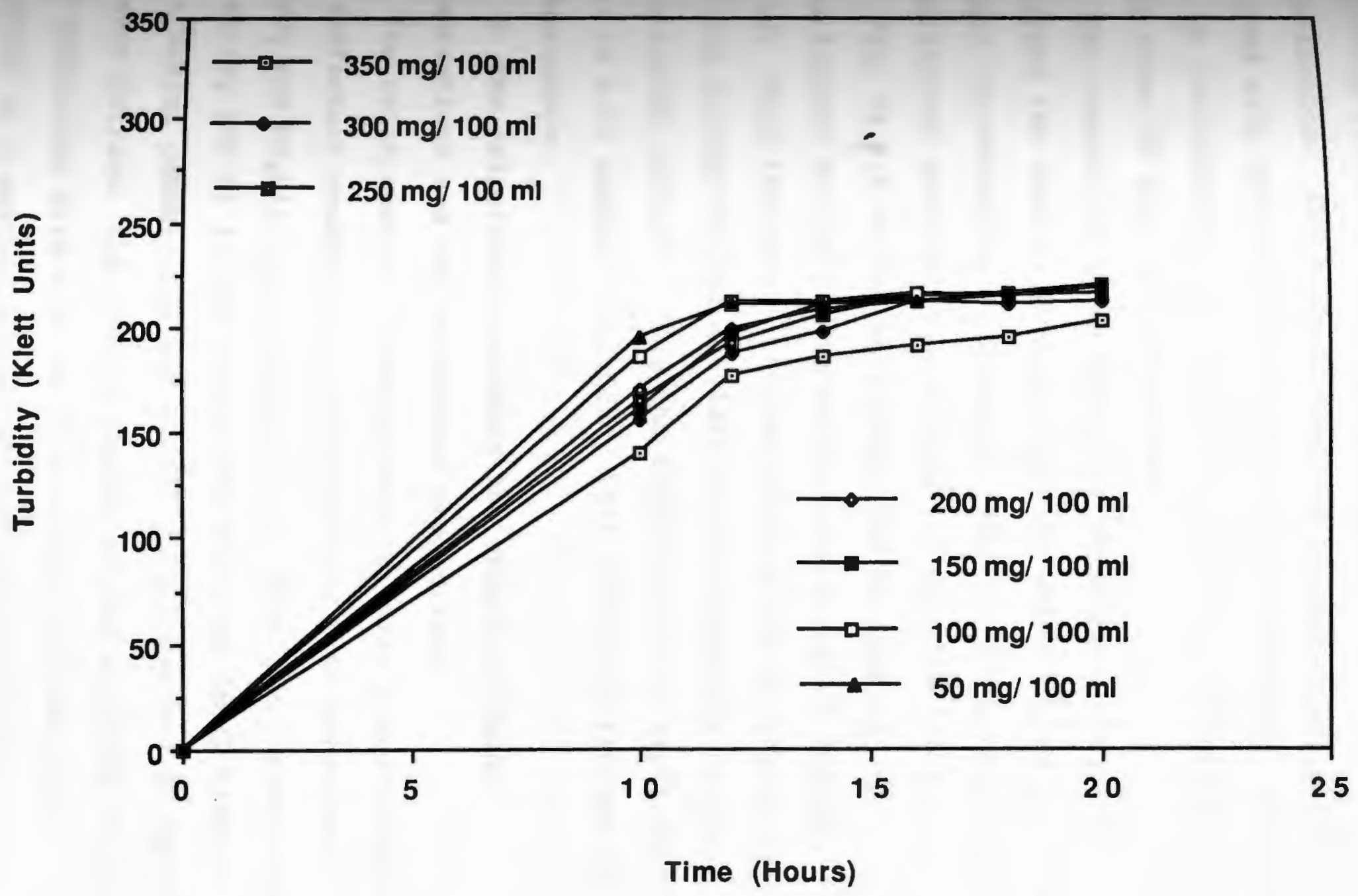


pduction increases with the increases of $\mathrm{N}$-sources pncentration. The highest yield of biosurfactant was bserved with $300 \mathrm{mg} / 100 \mathrm{ml}$ concentration of $\mathrm{NH}_{4} \mathrm{NO}_{3}$ after 22 hrs. of cultivation, and with $200 \mathrm{mg} / 100 \mathrm{ml}$ concentration of $\mathrm{NH}_{4} \mathrm{Cl}$ after $20 \mathrm{hrs}$. of cultivation.

The results obtained from this experiment (Fig 22)

confirmed the results obtained earlier which showed that the optimum concentration of $\mathrm{N}$-source $\left(\mathrm{NH}_{4} \mathrm{NO}_{3}\right)$ for growth and Bsurfactant production is $150 \mathrm{mg} / 100 \mathrm{ml}$ (Fig. $16 \mathrm{a}, \mathrm{b}$ ).

Fig. $24, a, b$ shows that, there was no loss of losurfactant activity with any concentration of $\left(\mathrm{NH}_{4} \mathrm{NO}_{3}\right)$ tested. This indicated that the loss of the biosurfactant from the culture during the late growth phase was due to a contaminated culture. Thus the results of this experiment (Fig. 24 a,b) suggest indirectly that the biosurfactant is Modegradable.

9. The relationship between the growth (biomass concentration) and the surfactant production:

The relationship between growth (biomass concentration) and surfactant production was studied for both $\mathrm{N}$-sources $\left(\mathrm{NH}_{4} \mathrm{NO}_{3}\right.$ and $\left.\mathrm{NH}_{4} \mathrm{Cl}\right)$, using nine $250 \mathrm{ml}$. side arm flasks, each pontaining $100 \mathrm{ml}$ filter sterilized modified basal mineral salts medium (MMSM) with $200 \mathrm{mg} / 100 \mathrm{ml}$ glucose and $20 \mathrm{mg} / 100$ ml yeast extract, the initial medium $\mathrm{pH}$ was adjusted to 7.0 , then inoculated with $1 \mathrm{ml}$ of $12 \mathrm{hrs}$. old inoculum and incubated on a psycrotherm at $200 \mathrm{rpm}$ and $30^{\circ} \mathrm{C}$ until it reach the required turbidity (biomass concentration). 
Fig. $24 \mathrm{a}, \mathrm{b}$, Effect of ammonium nitrate $\left(\mathrm{NH}_{4} \mathrm{NO}_{3}\right)$

concentration (50-350 with $50 \mathrm{mg} / 100 \mathrm{ml}$ increment) on biosurfactant production by Arthrobacter JIZAN-1 grown in batch culture on BMSM containing $0.2 \%$ glucose and $0.02 \%$ yeast extract at $200 \mathrm{rpm}$ and $30^{\circ} \mathrm{C}$ for $22 \mathrm{hrs}$, as determined by measuring the surface and interfacial tensions. 

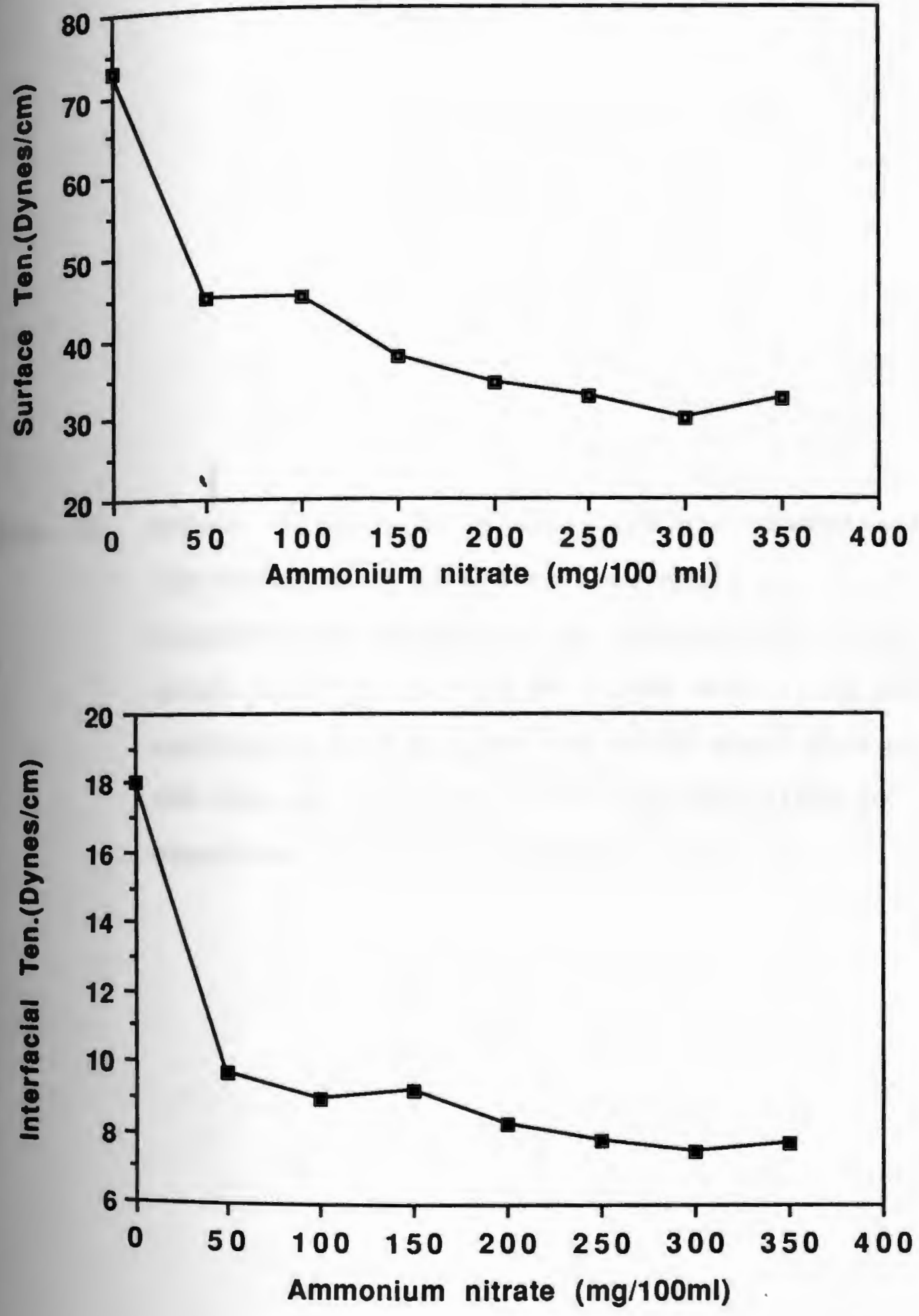
Fig. 25 Effect of ammonium chloride $\left(\mathrm{NH}_{4} \mathrm{Cl}\right)$ concentration (50-350 with $50 \mathrm{mg} / 100 \mathrm{ml}$ increment) on biosurfactant production by Arthrobacter JIZAN-1 grown in batch culture on filter sterilized BMSM containing $0.2 \%$ glucose and $0.02 \%$ yeast extract at $200 \mathrm{rpm}$ and $30^{\circ} \mathrm{C}$ for $20 \mathrm{hrs}$, as determined by measuring the surface tension. 


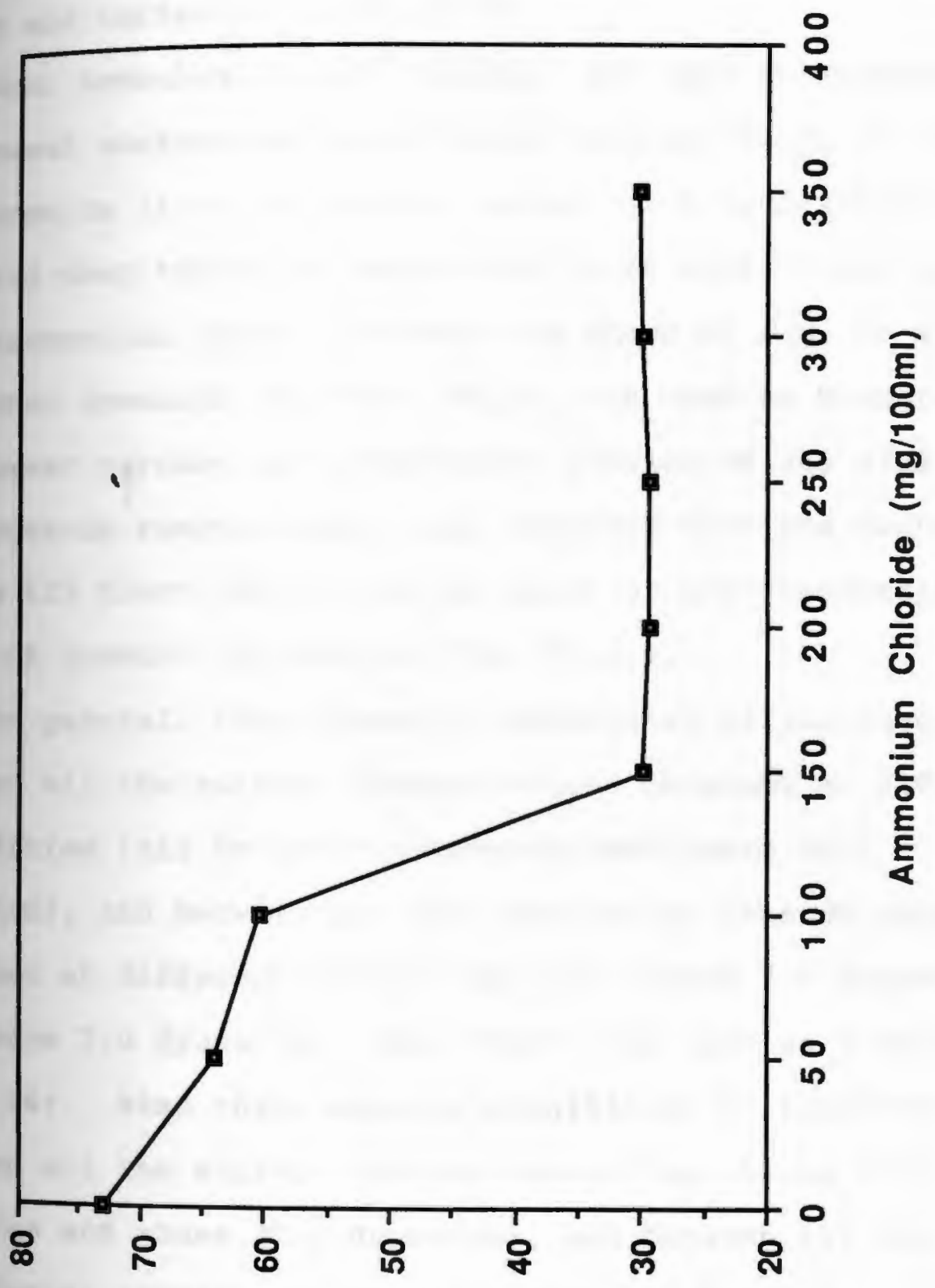

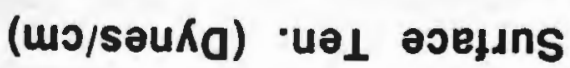


A turbidity range of 50-250 (with 25 klett units (crements) were used to establish the relation between growth and surfactant production.

When ammonium nitrate $\left(\mathrm{NH}_{4} \mathrm{NO}_{3}\right)$ was used as $\mathrm{N}$-source, the lowest surface and interfacial tension values 26.5 and 2.0 dynes/cm (i.e. the highest amount of biosurfactant) was abserved when turbidity reachs 200 klett units (i.e. at midexponential phase of growth) as shown by Fig. 26 a,b. When ammonium chloride $\left(\mathrm{NH}_{4} \mathrm{Cl}\right)$ was used as $\mathrm{N}$-source, the lowest surface and interfacial tension values 27.2 and 2.2 dynes/cm respectively, were observed when the turbidity reachs 125 klett units (i.e. at early or mid-exponential phase of growth) as shown by Fig. 27 a,b.

In general, there were no substantial differences between all the surface tension values obtained at different turbidities (all below 29.3 dynes/cm and above 26.5 dynes $(\mathrm{cm})$, and between all the interfacial tension values obtained at different turbidities (all below 4.0 dynes/cm and above 2.0 dynes $/ \mathrm{cm}$ ), when $\mathrm{NH}_{4} \mathrm{NO}_{3}$ was used as $\mathrm{N}$-source (Fig. 26). Also there were no significant differences between all the surface tension values (all below 29.8 dynes $/ \mathrm{cm}$ and above 27.1 dynes/ $\mathrm{cm}$ ), and between all the Mterfacial tension values (all below 4.5 dynes/cm and above 2.2 dynes $/ \mathrm{cm})$ when ammonium chloride $\left(\mathrm{NH}_{4} \mathrm{Cl}\right)$ was used as H-source at different turbidity (biomass concentration) (Fig. 27). 
Fig. $26 \mathrm{a}, \mathrm{b}$. The relationship between growth (biomass concentration) of Arthrobacter JIZAN-1 grown in batch culture on filter sterilized BMSM containing $0.2 \%$ glucose, $0.15 \% \mathrm{NH}_{4} \mathrm{NO}_{3}$ and $0.02 \%$ yeast extract at $200 \mathrm{rpm}$ and $30^{\circ} \mathrm{C}$, and the production of biosurfactant as determined by measuring surface and interfacial tensions. 

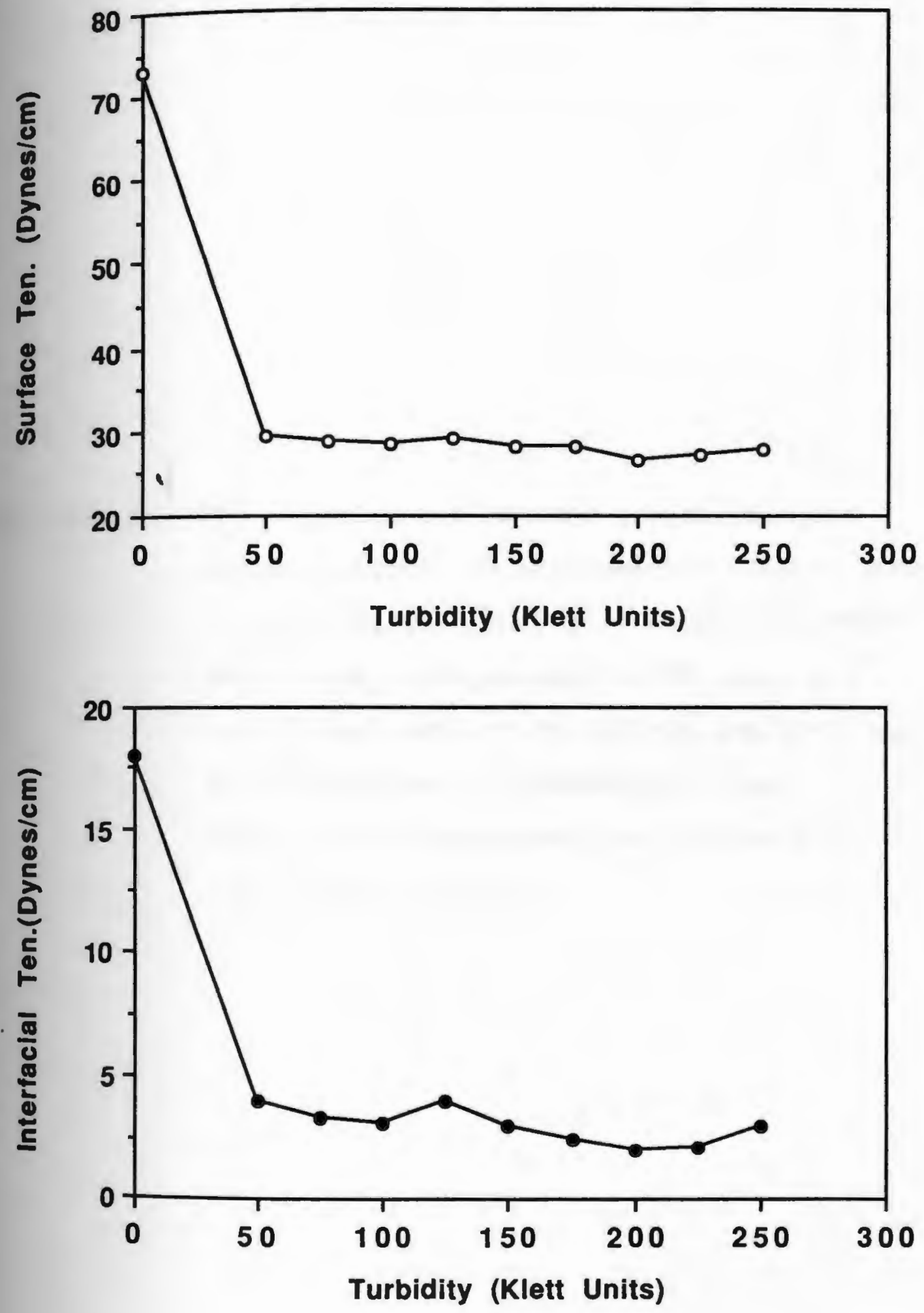
Fig. $27 \mathrm{a}, \mathrm{b}$ The relationship between growth (biomass concentration), of Arthrobacter JIZAN-1 grown in batch culture on filter sterilized BMSM containing $0.2 \%$ glucose, $0.15 \% \mathrm{NH}_{4} \mathrm{Cl}$ and $0.02 \%$ yeast extract at $200 \mathrm{rpm}$ and $30^{\circ} \mathrm{C}$, and the production of biosurfactant, as determined by measuring the surface and interfacial tensions. 

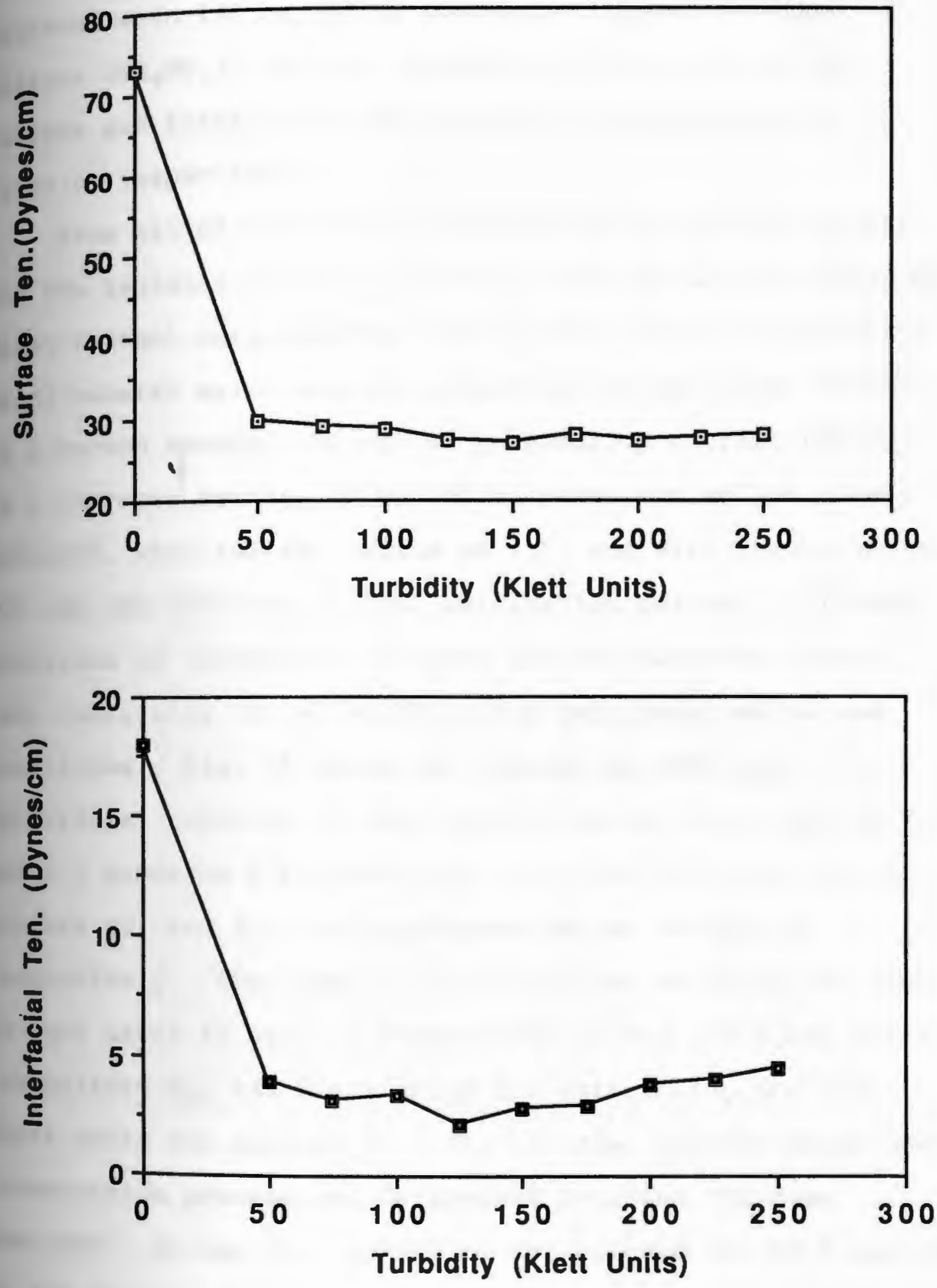
The highest yield of biosurfactant activity was bserved with $150 \mathrm{mg} / 100 \mathrm{ml}$ concentration of ammonium nitrate $\left(\mathrm{NH}_{4} \mathrm{NO}_{3}\right)$, because it gave the lowest values of surface and interfacial tension, 26.5 dynes/cm and 2.0 dynes/cm respectively.

From all of the previous experiments, the best growth for the isolated organism (JIZAN-1) and the highest yield of biosurfactant were observed with filter sterilized modified basal mineral salts medium containing $200 \mathrm{mg} / 100 \mathrm{ml}$ glucose as a carbon source, $150 \mathrm{mg} / 100 \mathrm{ml}$ ammonium nitrate $\left(\mathrm{NH}_{4} \mathrm{NO}_{3}\right)$ as a nitrogen source, $20 \mathrm{mg} / 100 \mathrm{ml}$ yeast extract as growth cofactor, with initial medium $\mathrm{pH} 7.0$, and with incubation at $200 \mathrm{rpm}$ and $30^{\circ} \mathrm{C}$ for $12 \mathrm{hrs}$. cultivation period. This was confirmed by incubation of three $250 \mathrm{ml-Erlenmyer} \mathrm{flasks}$ each containing $100 \mathrm{ml}$ of the above mentioned medium and conditions. Fig. 28 shows the average surface and interfacial tensions of each culture broth, which was 26.9 and 2.7 dynes/cm for culture \#1, 26.6 and 2.5 dynes/cm for culture \#2, and 27.1 and 2.6 dynes/cm for culture \#3 respectively. The growth (turbidity) was measured for each culture after $12 \mathrm{hrs}$. of incubation, it was 140 Klett units for culture \#1, 145 Klett units for culture \#2, and 125 Klett units for culture \#3. The residual glucose after the fermentation process was determined by using "Glucose analyzer", it was $75.7 \mathrm{mg} / 100 \mathrm{ml}$ for culture \#1, $53.0 \mathrm{mg} / 100$ $\mathrm{ml}$ for culture \#2, and $76.7 \mathrm{mg} / 100 \mathrm{ml}$ for culture \#3. 
Fig. 28 The reduction of surface and interfacial tensions of the whole culture broth by Arthrobacter JIZAN-1 grown in batch culture on filter sterilized BMSM containing $0.2 \%$ glucose, $0.15 \%$ $\mathrm{NH}_{4} \mathrm{NO}_{3}, 0.02 \%$ yeast extract and initial medium $\mathrm{pH}$ $7.0^{\circ}$ at $200 \mathrm{rpm}$ and $30^{\circ} \mathrm{C}$ for $12 \mathrm{hrs}$, as compared with deionized water as control.

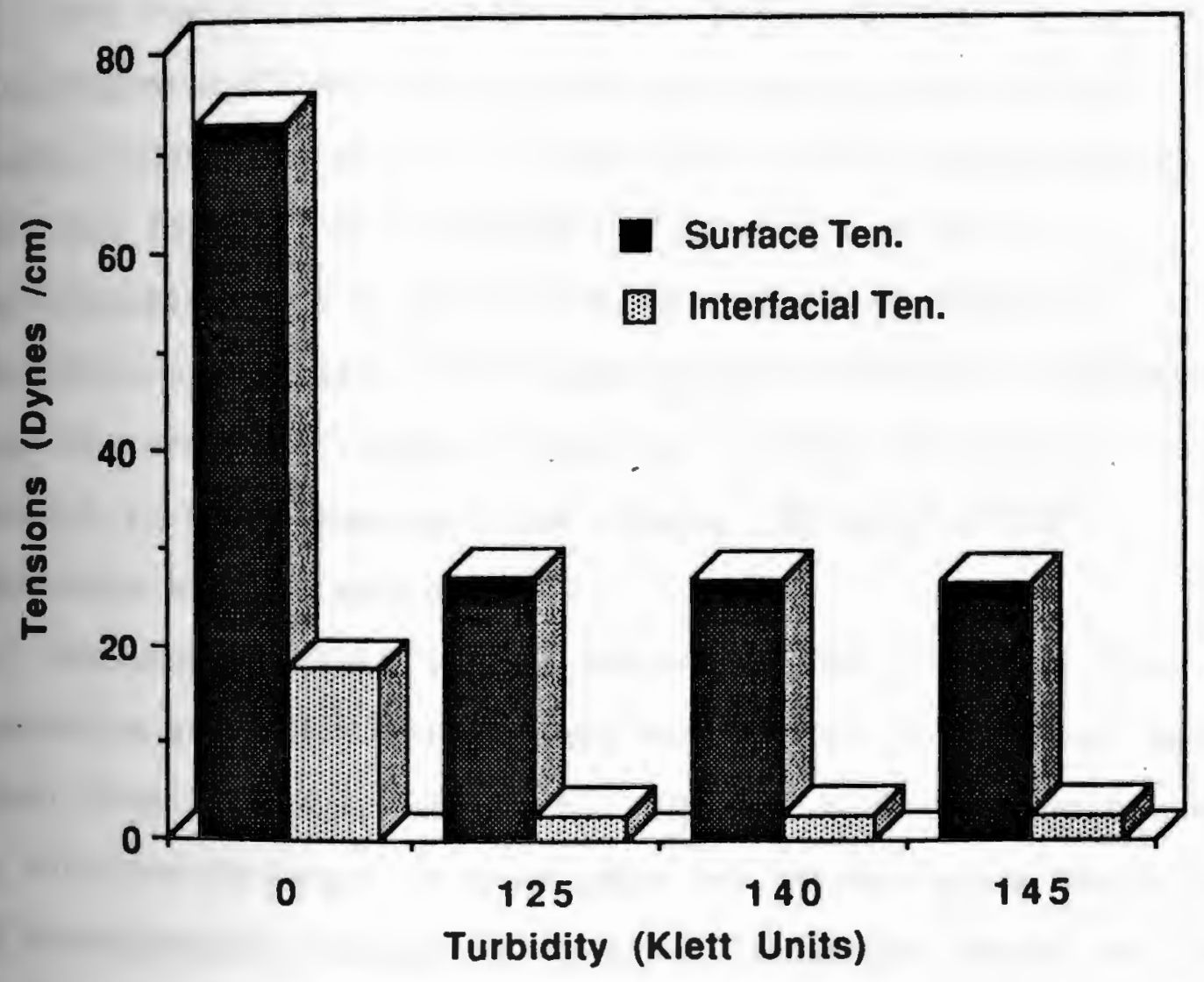


10. Identification of the microorganism:

The microorganism (JIZAN-1) was identified based on morphological, cultural, and biochemical tests as outlined in Bergey's Manual of determinative bacteriology (Buchannam and Ribbons, 1974). The morphological characteristics of the Llcroorganism (JIZAN-1) were examined by utilizing gram stains of a $15 \mathrm{hr}$. old culture grown on a nutrient agar slant. The organism was found to be gram variable, and cells were irregular in shape and size, fairly thick and rounded in the end, and found in v-forms and palisade. The reduction of nitrate to nitrite by the croorganism JIZAN-1 was tested by using Bacto nitrate broth, inoculated with a $15 \mathrm{hrs}$. NA culture and incubated at $30^{\circ} \mathrm{C}$ for $24 \mathrm{hrs}$. The standard sulphanilic acid- $\alpha$ Faphthalamine in $5 \mathrm{~N}$ acetic acid reagent was used for detection of nitrite. The absence of a red color indicated that the organism (JIZAN-1) did not reduce nitrate to nitrite in this standard time frame. Delayed nitrate reduction was not tested.

Anaerobic growth of the microorganism (JIZAN-1) was tested by stab inoculation into reinforced clostridial agar (RCA) from a $15 \mathrm{hrs.} \mathrm{NA} \mathrm{slant.} \mathrm{The} \mathrm{RCA} \mathrm{tube} \mathrm{was} \mathrm{incubated}$ at $30^{\circ} \mathrm{C}$ for $24 \mathrm{hrs}$. and examained for growth along the line of inoculation. There was no growth observed along the line of inoculation to the bottom of the stab, which indicated that JIZAN-1 can not grow under anaerobic conditions. 
The motility of the microorganism (JIZAN-1) was tested, using Bacto motility test medium (semi-solid media), by stab inoculation the center of the media from a $15 \mathrm{hrs}$. NA slant culture, which then incubated at $30^{\circ} \mathrm{C}$ for $24 \mathrm{hrs}$. There was no diffuse horizontal growth observed from the line of inoculation indicating that JIZAN-1 is a non-motile organism.

The ability of the microorganism (JIZAN-1) to hydrolyze gelatin was tested by using nutrient gelatin plate (nutrient agar +48 gelatin), inoculated from a $15 \mathrm{hrs}$. NA slant culture and incubated at $30^{\circ} \mathrm{C}$ for $24 \mathrm{hrs}$. The plate was flooded with acidified $\mathrm{HgCl}_{2}$ to precipitate the unhydrolyzed gelatin. There was no clear zone surrounding the growing bacteria indicating that JIZAN-1 did not hydrolyze gelatin. The ability of the microorganism (JIZAN-1) to hydrolyze starch was tested by growth on nutrient agar starch plate (Bacto starch agar plate), inoculated with a 15 hrs. old NA culture, and incubated at $30^{\circ} \mathrm{C}$ for $24 \mathrm{hrs}$. The plate was flooded with "Logol's" iodine solution to turns the pahydrolyzed starch to blue color. There was no colorless zone observed surrounding the growing bacteria indicating that JIZAN-1 did not hydrolyze starch.

The production of acid and gas from glucose was Aetermined in Bacto phenol red broth with $1 \%$ glucose, pontaining Durham tube which inoculated from a $15 \mathrm{hrs}$. NA slant culture, then incubated at $30^{\circ} \mathrm{C}$ for $24 \mathrm{hrs}$. Acid production by the microorganism (JIZAN-1) was indicated by 
the yellow color change of phenol red, but there was no gas yoduced in the Durham tube. These results indicate that JZAN-1 glucose metabolism is not fermentative.

Temperature range between $25-35^{\circ} \mathrm{C}$ (at $5^{\circ} \mathrm{C}$ increments)

was used to determine the optimum temperature for growth. The optimum temperature for growth was found to be $30^{\circ} \mathrm{C}$. The results obtained from morphological, cultural, and techemical tests indicating that the isolated microorganism (JIZAN-1) which can assemble the biosurfactant from carbohydrate (glucose) substrate, is an Arthrobacter gpecies. 
II-Determination of proteins, lipids and carbohydrates acreted into the culture broth during the fermentation process.

\section{A. Proteins :}

one of the most widely used methods for the etermination of protein in solutions is based on the teraction of proteins with phenol and copper under alkaline conditions (Pomeranz and Meloan, 1978).

Proteins excreted into the culture broth during the fermentation process by Arthrobacter (JIZAN-1) were determined by the Folin phenol reagent method of Lowry et al. (1951) based on beef serum albumin as standard.

The protein standard curve shown in Fig. 29 was used for determination of the protein concentration in $1 \mathrm{mI}$ culture broth sample. The result obtained from the standard curve indicate that, a negligible amount of protein (45 $\mu \mathrm{g} / \mathrm{ml})$ was excreted into the culture broth. This method is relatively specific, since few substances encountered in plological materials cause serious interference (Solecka et al., 1968).

\section{B. Lipids :}

Lipids which excreted into the culture broth during fermentation were identified by thin layer chromatography (TLC), and their constituent fatty acids were determined by gas chromatography (GC).

Lipids were extracted from $300 \mathrm{ml}$ of culture medium using a continuous liquid-liquid extractor with diethyl 
Fig. 29 Protein standard curve which was prepared by the Folin phenol reagent method based on beef serium albumin as a standard and utlized for determination of protein concentration in $1.0 \mathrm{ml}$ culture broth sample.

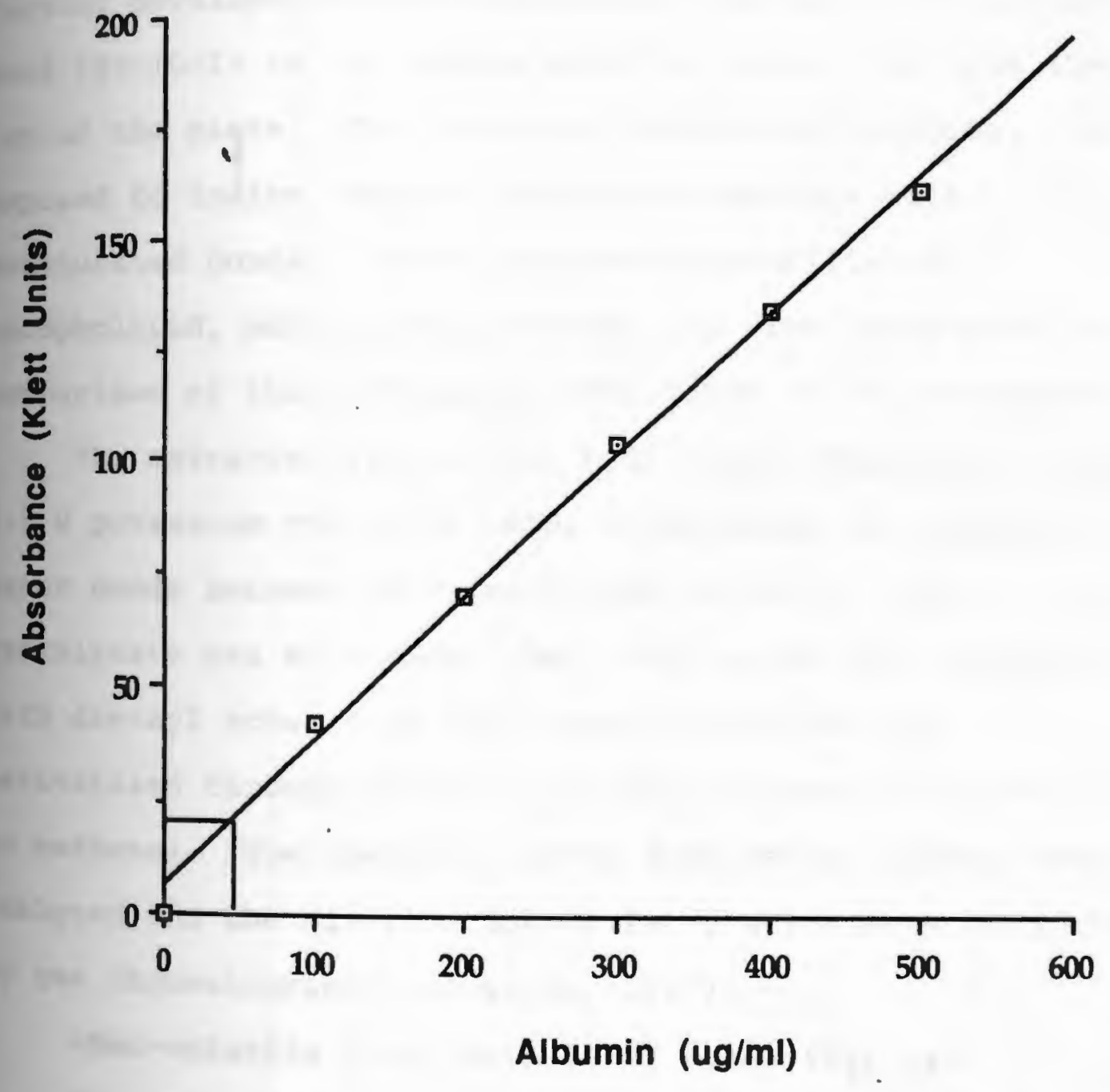


ether as the solvent for $12 \mathrm{hrs}$. The solvent was removed by vacuum evaporation, leaving an oily residue.

Iipids from the fermentation broth and two standards (polar and non-polar lipids) were spotted on to Fisher silica gel $G$ type TLC plate with a $\mathrm{CaSO}_{4}$ binder, first leveloped by using diisopropyl ether/acetic acid (96:4) as the mobile phase for 2-3 cm up from the base line, and further developed by petroleum ether/diethyl ether/acetic acid $(90: 10: 1)$ as the mobile phase to about $1 \mathrm{~cm}$ from the top of the plate. The plate was dried with nitrogen, then exposed to iodine vapours to detect compounds with unsaturated bonds. Three spots were identified as Mospholipid, mono or diglycerides and free fatty acids by comparison of their Rf values with those of the standards. The extracted lipids were hydrolyzed (saponified) using $0.5 \mathrm{~N}$ potassium hydroxide $(\mathrm{KOH})$ in methanol to cleve the ester bonds between fatty acids and glycerol. The hydrolyzate was acidified, then fatty acids were extracted with diethyl ether from the reaction mixture, and derivatized through methylation using borontrifluoride $\left(\mathrm{BF}_{3}\right)$ in methanol. The resultant fatty acid methyl esters were analyzed and the distribution of fatty acids were determined by gas chromatography (Gunstone, 1967).

-Non-volatile long chain fatty acids $\left(\mathrm{C}_{16}-24\right)$ :

Fig. 30 and Fig. 31 shows the gas chromatographic profile of the non-volatile long chain fatty acid methyl esters obtained from GLC-10 and PUFA-1 standards 
Fig. 30. Gas chromatographic profile of fatty acid methyl for determination of the relative retention times.

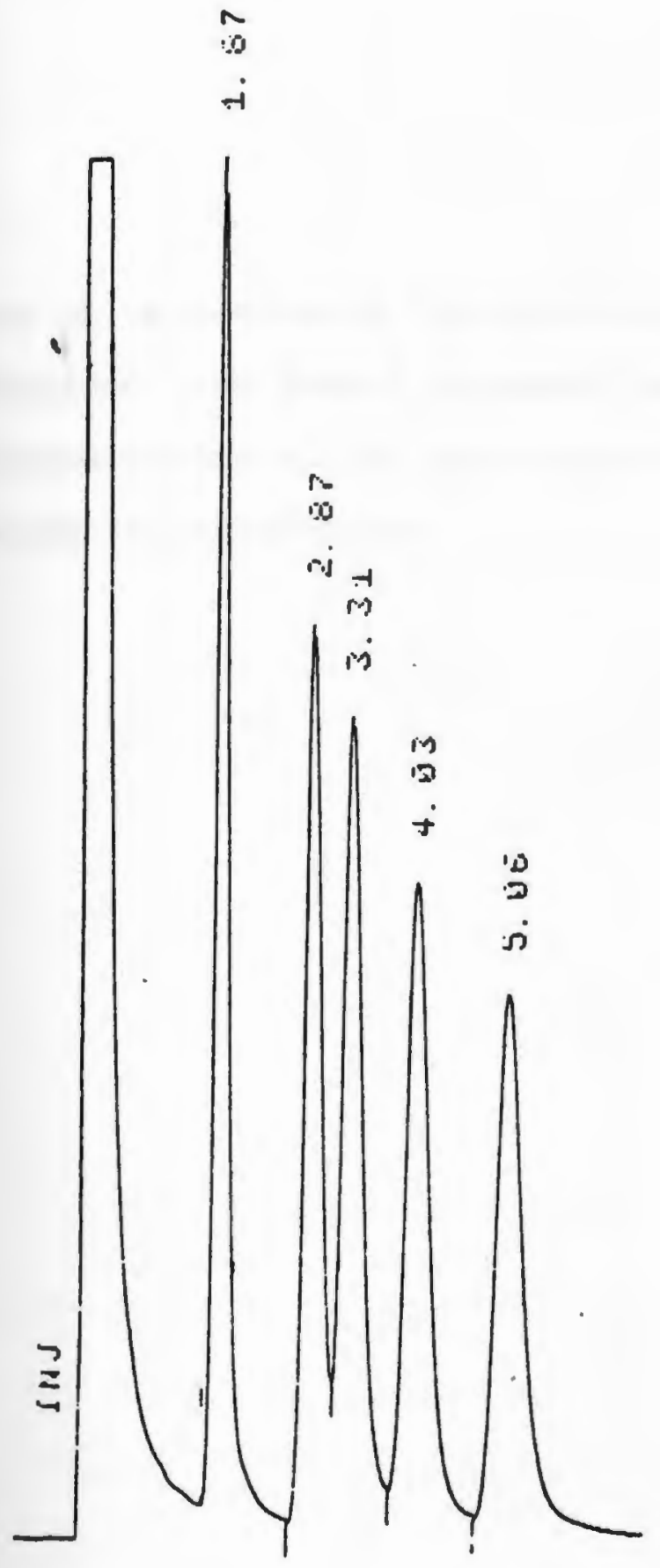


Fig. 31 Gas chromatogram of the fatty acid methyl esters obtained from PUFA-1 standard, which was used for determination of the non-volatile long chain fatty acids retention times. 

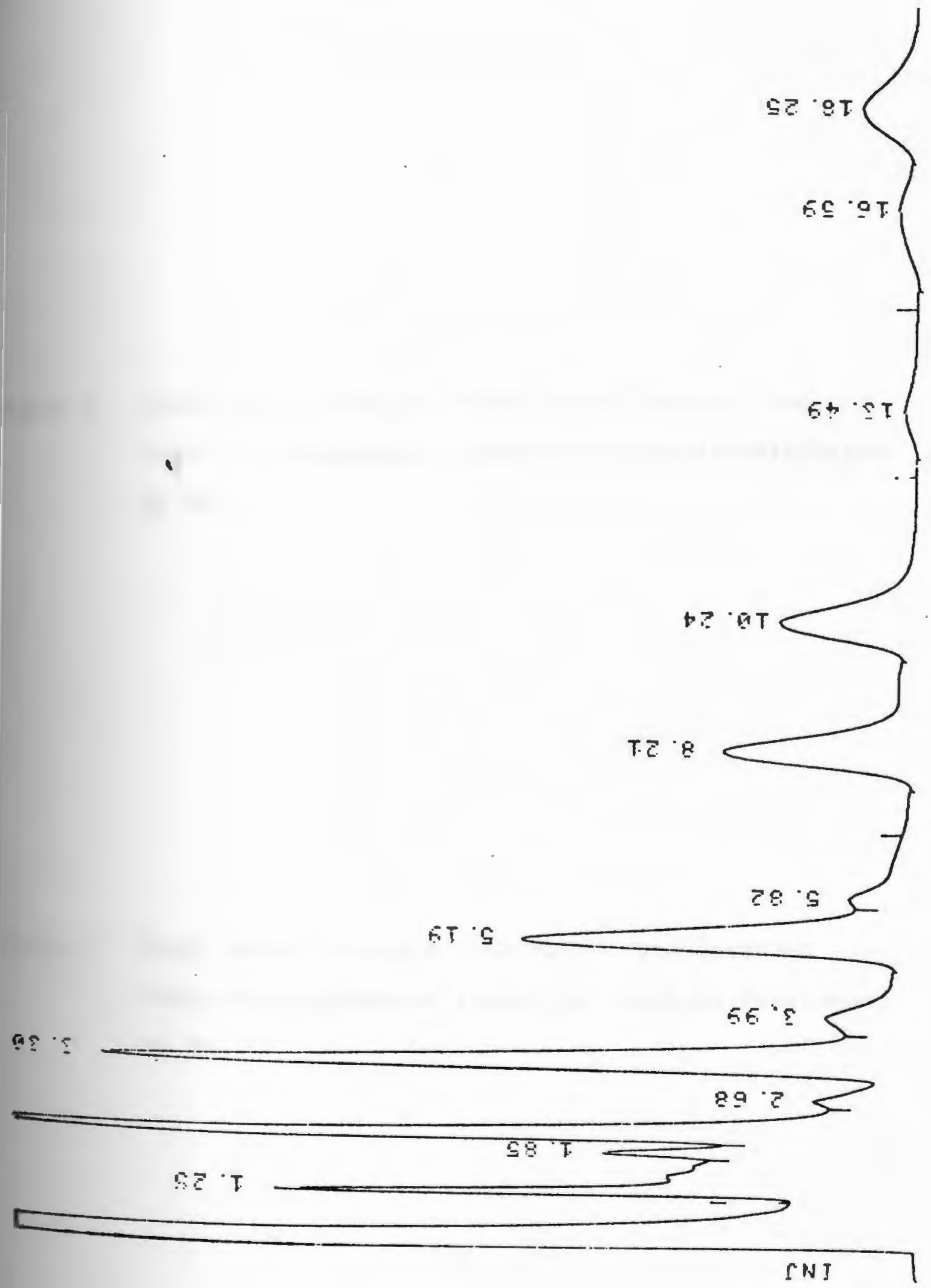
Table 6 Fatty acids obtained from GLC-10 standard and their correspondence retention times as determined by GC.

Table 7 Fatty acids obtained from PUFA-1 standard and their correspondence retention times as determined by GC. 


\begin{tabular}{lll}
\hline RT & $\begin{array}{c}\text { \% of Carbons } \\
\text { \# of Double Bonds }\end{array}$ & Fatty Acids \\
\hline 1.87 & $16: 0$ & Palmitic \\
2.87 & $18: 0$ & Stearic \\
3.31 & $18: 1 w 9$ & Oleic \\
4.03 & $18: 2 w 6$ & Linoleic \\
5.06 & $18: 3 w 3$ & Linolenic \\
\hline
\end{tabular}

\begin{tabular}{lll}
\hline RT & $\begin{array}{l}\text { *f Carbons } \\
\text { of Double Bonds }\end{array}$ & Fatty Acids \\
1.25 & $14: 0$ & Myristic \\
1.85 & $16: 0$ & Palmitic \\
2.16 & $16: 1$ & Palmitoleic \\
3.30 & $18: 1 w 9$ & Oleic \\
3.99 & $18: 2 w 6$ & Linoleic \\
5.19 & $20: 1$ & Gondoic \\
8.21 & $22: 1$ & Erucic \\
10.24 & $20: 5 w 3$ & Eicosapentaenoic \\
13.49 & $24: 1$ & Nervonic \\
16.59 & $22: 5 \mathrm{w} 3$ & Docosahexaenoic \\
18.22 & $22: 6 \mathrm{w} 3$ & \\
\hline
\end{tabular}


espectively. A good and clear separation was obtained for both GLC-10 and PUFA-1 standards, and they were used for petermination of the retention times of the non-volatile long chain fatty acids. The retention times and their responding fatty acids obtained from GLC-10 and PUFA-1 tandards were listed respectively in table 6 and table 7 . Eig. 32 shows the gas chromatographic profile of the non-volatile long chain fatty aicds from culture broth after saponification and methylation. Ten peaks (fatty acids) were identified based on their retention times, and the eight major peaks were identified as $\mathrm{C}_{14: 0}, \mathrm{C}_{16: 0}$, $\mathrm{C}_{16: 1} \cdot \mathrm{C}_{18: 0} \cdot \mathrm{C}_{18: 1 \mathrm{~W} 9}, \mathrm{C}_{18: 2 \mathrm{~W} 6}, \mathrm{C}_{20: 5 \mathrm{~W} 3}$ and $\mathrm{C}_{22: 5 \mathrm{~W} 3} \cdot$ Palmitic acid $\left(\mathrm{C}_{16: 0}\right)$ was found to be the predominant fatty acid as shown by Fig. 32. The retention times and their eorresponding fatty acids obtained from the culture broth sample are summerized in table 8 .

\section{-Non-volatile short chain fatty acids $\left(C_{8}-C_{16}\right)$ :}

Fig. $33 a, b$ shows the gas chromatographic profile of the non-volatile short chain fatty acid methyl esters obtained from the standards GLC-30 and GLC-70. A good separation and reproducable results were obtained from both standards. Fatty acids and their corresponding retention times obtained from GLC-30 and GLC-70 standards were listed in table 9 and table 10 respectively. Fig. 33 c represents the gas chromatogrpahic profile of the non-volatile short chain fatty acids obtained from the culture broth sample after saponification and methylation. Five major peaks were 
Fig. 32

Gas chromatographic profile of non-volatile long chain fatty acids obtained from the culture broth after extraction by ethyl ether, saponification by $\mathrm{KOH}$ in methanol and derivatization by $\mathrm{BF}_{3}$ methanol.

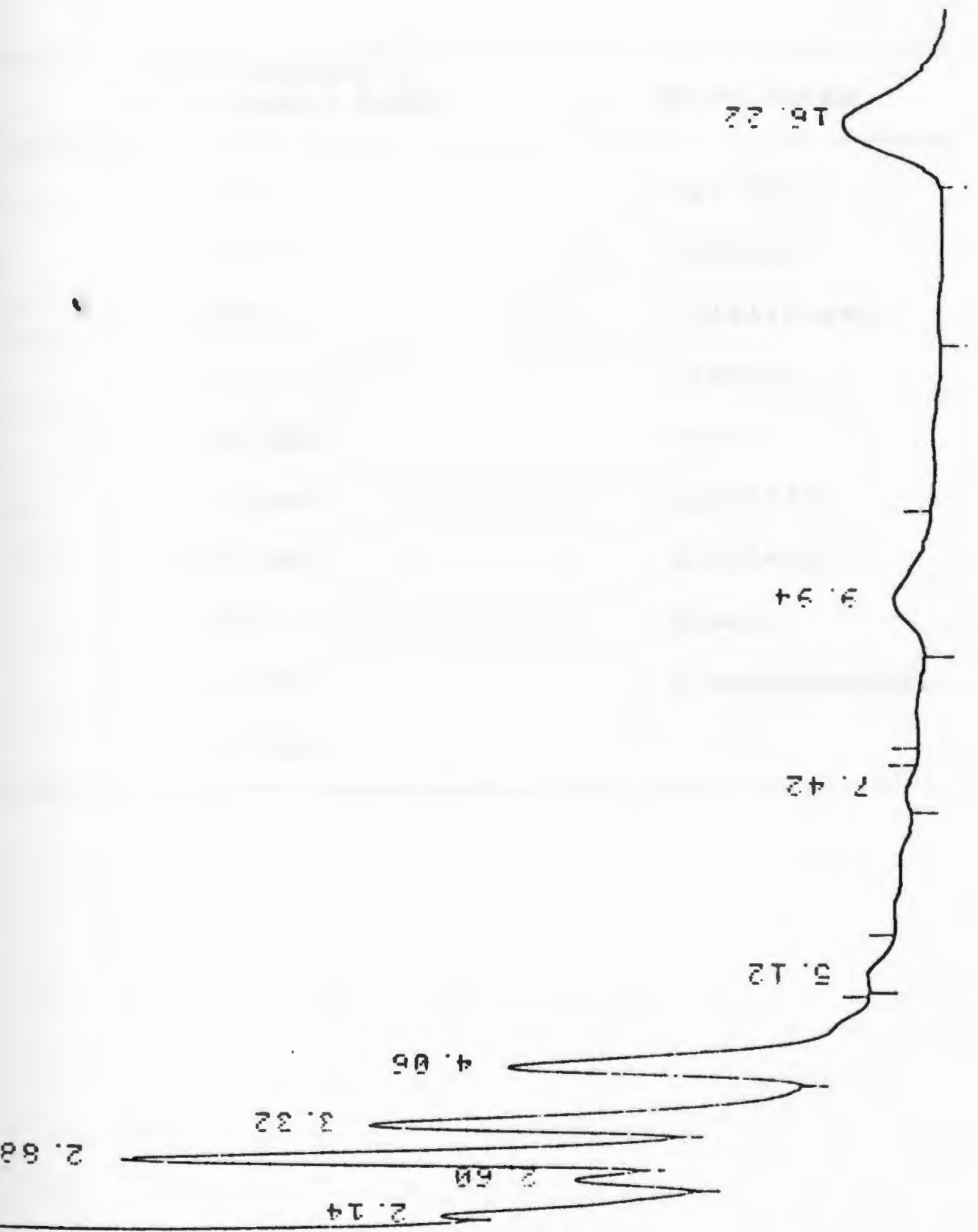

$98 . T$ 
Table. 8 Non-volatile by long chain fatty acids obtained from Arthrobacter JIZAN-1 culture broth and their correspondence retention times as determined by GC.

\begin{tabular}{lll}
\hline RT & $\begin{array}{c}\text { \# of Carbons } \\
\text { \# of Double Bonds }\end{array}$ & Fatty Acids \\
\hline 1.26 & $14: 0$ & Myristic \\
1.87 & $16: 0$ & Palmitic \\
2.14 & $16: 1$ & Palmitioleic \\
2.88 & $18: 0$ & Stearic \\
3.32 & $18: 1 \mathrm{w} 9$ & Oleic \\
4.06 & $18: 2 \mathrm{w} 6$ & Linoleic \\
5.12 & $18: 3 \mathrm{w} 3$ & Linolenic \\
7.42 & $22: 1$ & Erucic \\
9.94 & $20: 5 \mathrm{w} 3$ & Eicosopentaenoic \\
16.22 & $22: 5 \mathrm{w} 3$ & \\
\hline
\end{tabular}


Fig. $33 \mathrm{a}, \mathrm{b}$ Gas chromatogram of the non-volatile short chain fatty acid methyl esters obtained from GLC-30 and GLC-70 standards.

Fig. 33 c Gas chromatographic profile of the non-volatile short chain fatty acid methyl esters obtained from the culture broth after extraction by ethyl ether, saponification by $\mathrm{KOH}$ in methanol and derivatization by $\mathrm{BF}_{3}$ in methanol 


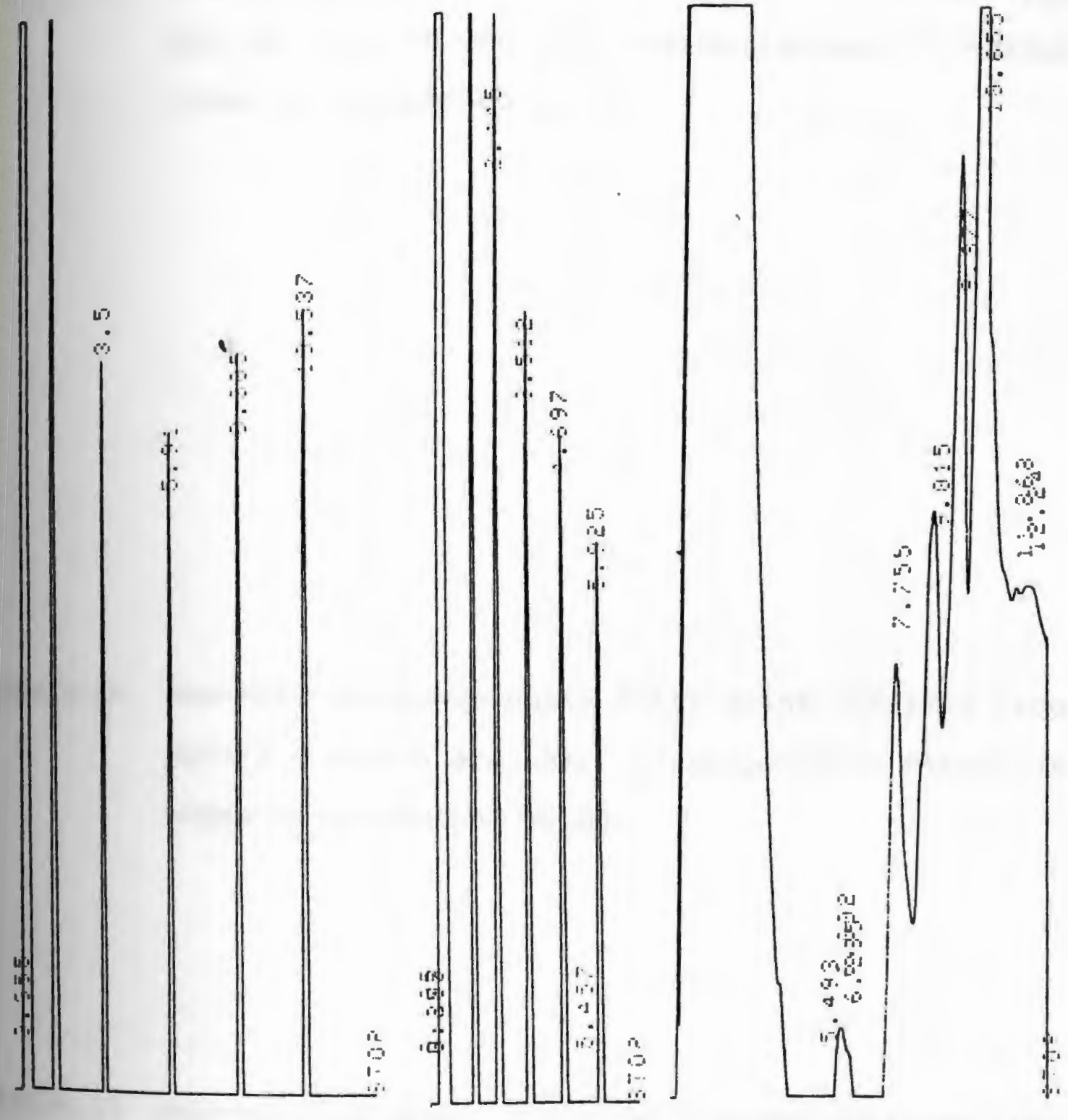


Table 9 Non-volatile short chain fatty acids obtained from GLC-30 standard and their correspondence retention times as determined by GC.

Table 10 Non-volatile short chain fatty acids obtained from GLC-70 standard and their correspondence retention times as determined by GC.

Table 11 Non-volatile short chain fatty acids obtained from Arthrobacter JIZAN-1 culture broth after extraction, saponification and derivatization and their correspondence retention times as determined by GC. 


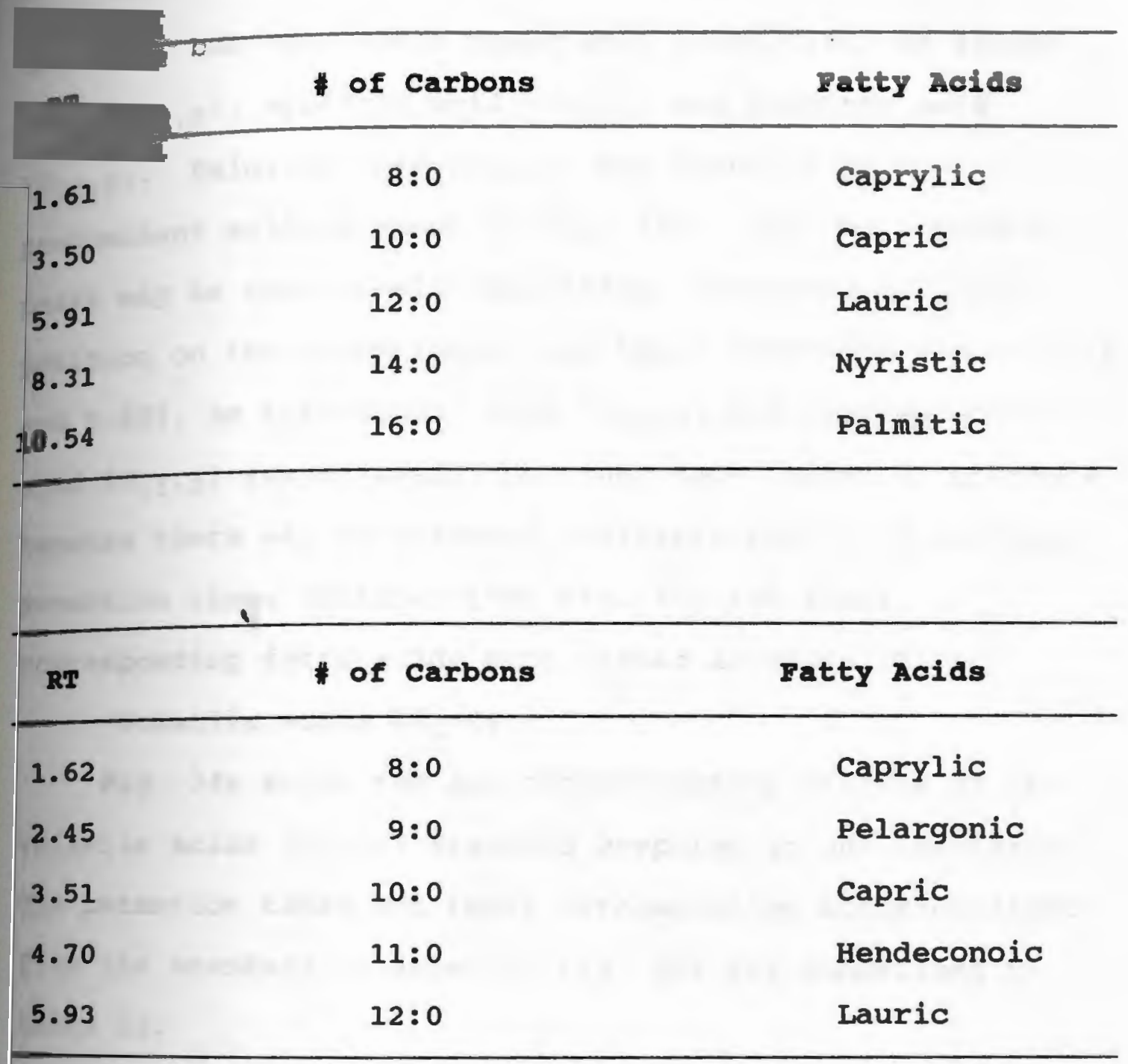

ratty Acids

6.01

$12: 0$

Lauric

7.76

Unknown

9.02

$14: 0$

Myristic

9.88

-

Unknown

10.66

$16: 0$

Palmitic 
btected, but only three peaks were identified, as lauric acid $\left(C_{12: 0}\right)$, myristic acid $\left(C_{14: 0}\right.$, and palmitic acid $\left(c_{16: 0}\right)$. Palmitic acid $\left(c_{16: 0}\right)$ was found to be the predominant acid as shown by Fig. 33c. The two unknowns peaks may be tentatively identified, according to their position on the chromatogram and their retention times 17.76 and 9.88$)$, as tridecanoic acid $\left(\mathrm{C}_{13}: 0\right)$ and pentadecanoic acid $\left(C_{15: 0}\right)$ respectively, but they were listed as unknowns because there was no standard available for $c_{13: 0}$ or $c_{15: 0}$. Retention times obtained from Fig. $31 \mathrm{C}$ and their arresponding fatty acids were listed in table 11.

-Volatile acids $\left(C_{2}-C_{7}\right)$ :

Fig. $34 a$ shows the gas chromatography profile of the volatile acids $\left(\mathrm{C}_{2}-\mathrm{C}_{7}\right)$ standard prepared in our laboratory. The retention times and their corresponding acids obtained from the standard as shown by Fig. 34 a are summarized in table 12 .

Fig. $34 \mathrm{~b}$ shows the gas chromatographic profile of the volatile acids $\left(C_{2}-C_{7}\right)$ excreted to the culture broth during the fermentation process. Seven major peaks (volatile acids) were detected, six peaks $\left(C_{2}-C_{7}\right)$ were identified based on their retention times. The other major peak detected before the other volatile acids $c_{2}-c_{7}$ which had a retention time 0.43 may be formic acid. The retention times and their corresponding acids obtained from Fig. $34 \mathrm{~b}$ are listed in table 13. 
Fig. 34 a Gas chromatogram of the volatile acids $\left(C_{2}-C_{7}\right)$ obtained from the standard.

Fig. 34 b Gas chromatographic profile of the volatile acids $\left(\mathrm{C}_{2}-\mathrm{C}_{7}\right)$ obtained from Arthrobacter JIZAN-1 culture broth. 

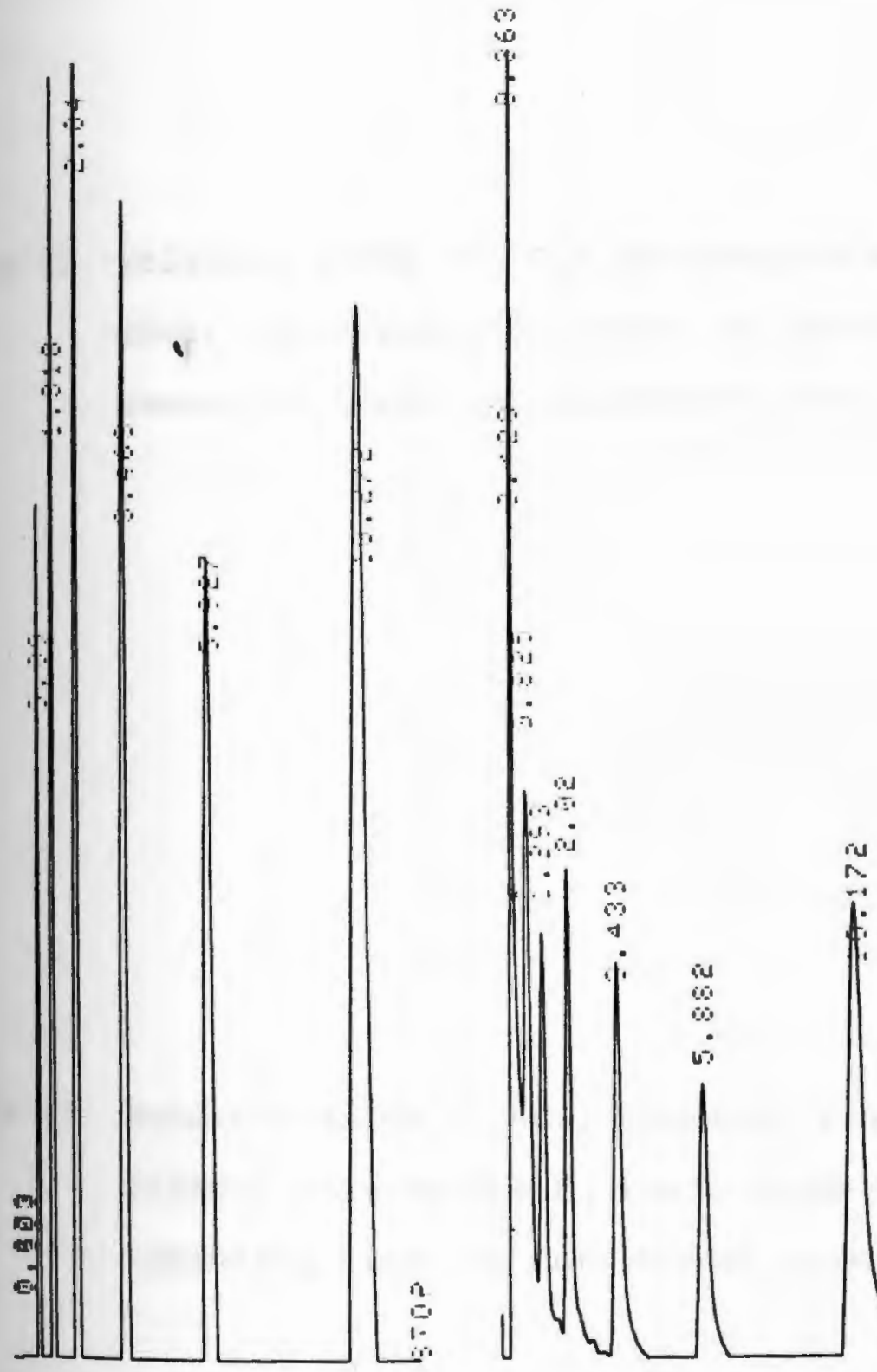

$-161-$ 
Table 12 Volatile acids $\left(\mathrm{C}_{2}-\mathrm{C}_{7}\right)$ obtained from the standard, thęir correspondence number of carbons and retention times as determined by GC.

Table 13 Volatile acids $\left(C_{2}-C_{7}\right)$ obtained from Arthrobacter JIZAN-1 culture broth, their number of carbons and retention times as determined by GC. 


\begin{tabular}{lll}
\hline RT of Carbons & Fatty Acids \\
\hline 0.89 & 2 & Acetic \\
1.32 & 3 & Propionic \\
2.04 & 4 & Butyric \\
3.46 & 5 & Valeric \\
5.93 & 6 & Caproic \\
10.27 & 7 & Enanthic \\
\hline
\end{tabular}

\begin{tabular}{|ccl} 
RT & \# of Carbons & Fatty Acids \\
0.83 & 2 & Acetic \\
1.29 & 3 & Propionic \\
2.02 & 4 & Butyric \\
3.43 & 5 & Valeric \\
5.88 & 6 & Caproic \\
10.17 & 7 & Enanthic \\
\hline
\end{tabular}




\section{c. Carbohydrates:}

Carbohydrates excreted into the culture broth during the fermentation process by Arthrobacter JIZAN-1 were determined by utilizing two different methods: Lipids were extracted from the culture broth sample before carbohydrate determination to prevent interference.

1-Colorimetric methods:

Reducing sugars excreted into the culture broth were determined by utilizing two colormetric methods. Glucose was used as a standard to prepare the standard curve in both methods.

Anthrone-sulfuric acid:

Anthrone-sulfuric acid method (Dreywood, 1946) was used for determination of total reducing sugars based on glucose as standard. A Klett-Summerson photoelectric colorimeter equipped with red filter was used for measuring the Intensity of the blue-green color formed from the reaction of anthrone-sulfuric acid with reducing sugars.

The glucose standard curve shown by Fig. 35 was utilized for determination of total reducing sugar concentration in $0.2 \mathrm{ml}$ of culture broth. The total reducing sugar concentration obtained by the Anthrone method was $240 \mathrm{\mu g} / \mathrm{ml}$. Residual glucose $(50 \mathrm{ug} / \mathrm{ml})$ was determined by the glucose oxidase reaction indicating that other reducing sugar were secreted into the culture broth. The Anthrone-sulfuric acid method gives the best results when applied to pure solution of hexose sugars or 
Fig. 35 Glucose standard curve, which was prepared by anthrone-sulfuric acid method and utilized for determination the reducing sugar concentration in $0.2 \mathrm{mI}$ of Arthrobacter JIZAN-1 culture broth sample.

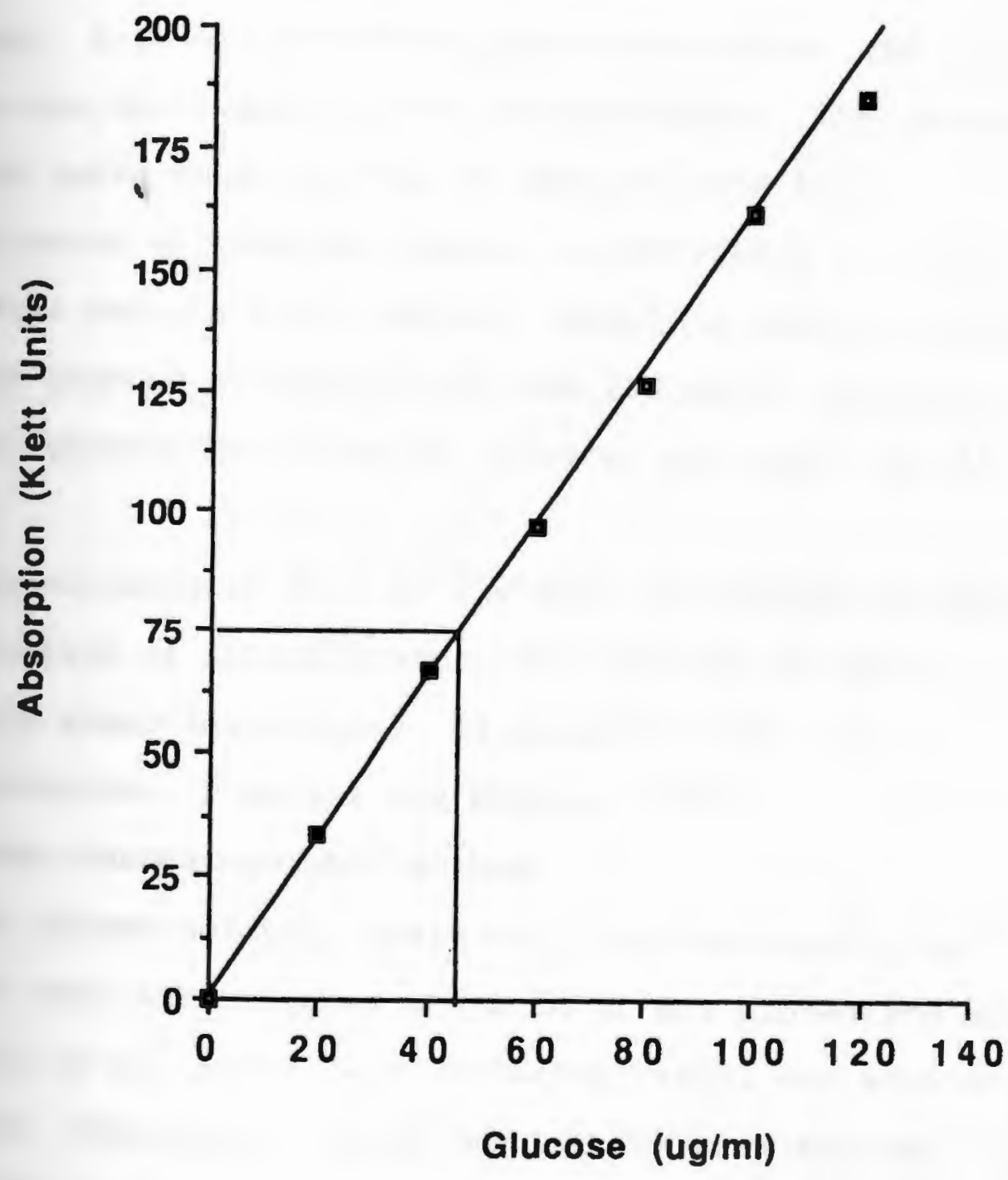


their polymers which produced the characteristic blue-green color (Pomeranz and Meloan, 1978).

Phenol-sulfuric acid method

Phenol-sulfuric acid method (Dubois et al., 1956), was also used for determination of total reducing sugars wxcreted into the culture broth by Arthrobacter JIZAN-1 during the fermentation process based on glucose as tandard. A Lambda 4B UV/VIS spectrophotometer was used to measure the absorbance at $485 \mathrm{~nm}$ wavelength. The glucose standard curvę shown in Fig. 36 was utilized for determination of reducing sugars concentration in $0.2 \mathrm{ml}$ lipid free culture broth sample. Reducing sugars obtained from the glucose standard curve was $280 \mu \mathrm{g} / \mathrm{ml}$, whereas residual glucose monitored by "glucose analyzer" was 50 $\mu \mathrm{g} / \mathrm{ml}$.

Phenol-sulfuric acid is a widely applicable method for determination of carbohydrates, all classes of sugars, Including sugar derivatives, oligosaccharides, and polysaccharides (Pomeranz and Meloan, 1978).

2-Gas chromatographic method:

Gas chromatographic analysis of carbohydrates has received much attention as a result of the pioneering work of Sweely et al. (1963) with trimethyl silyl derivatives. Two steps, hydrolysis and derivatization were applied first to the dry mixture of lipid free culture broth as described in the Materials and Methods (Chapter II) before analyzing by $\mathrm{GC}$. 
Fig. 36 Glucose standard curve, which was prepared by phenol-sulfuric acid method and utlized for determination the reducing sugar concentration in $0.2 \mathrm{ml}$ of Arthrobacter JIZAN-1 culture broth sample.

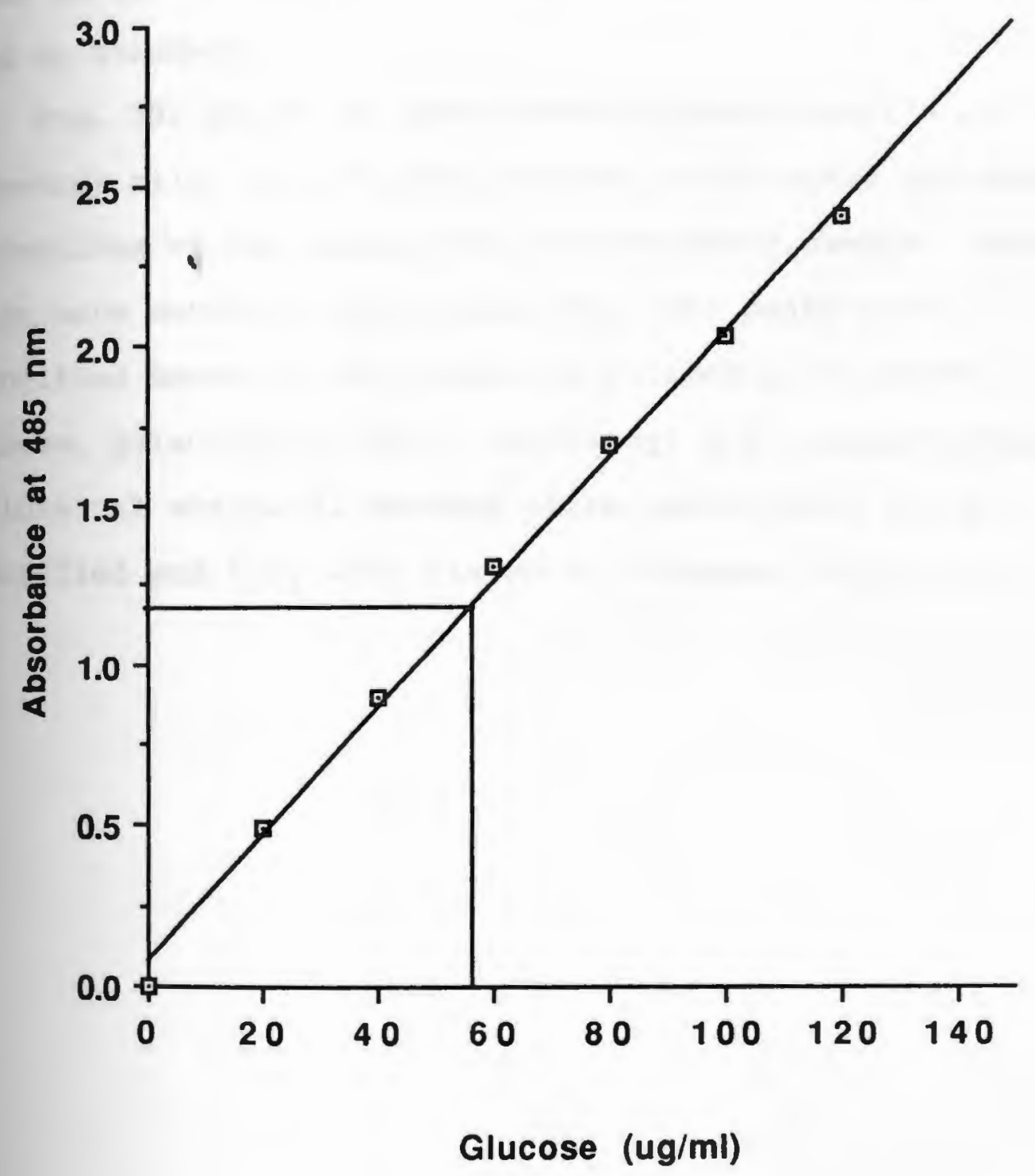


Fig. $37 \mathrm{a}, \mathrm{b}, \mathrm{c}$, Fig $38 \mathrm{a}, \mathrm{b}, \mathrm{c}$ and Fig. $39 \mathrm{a}, \mathrm{b}$ shows the gas matographic profile of sugars and sugar derivatives which were used separately as standards for determination the relative retention times. Retention times and their rresponding sugars or sugar derivatives were listed in table 14 for all sugars and sugar derivatives which were used as standard.

Fig. 39c shows the gas chromatographic profile of the trimethyl silyl derivatives obtained from sugars and sugar derivatives of the lipid free culture broth sample. Seven peaks were detected by GC, but only four peaks were identified based on their retention times as rhamnose, glucose, galacturonic acid, and phenyl B.D. glucopyramoside as internal standard, whereas three peaks could not be identified and they were listed as unknowns (table 15). 
Fig. $37 \mathrm{a}, \mathrm{b}$ and $\mathrm{c}$ Gas chromatograms obtained for xylose

(a), ribose (b) and rhamnose (c) after derivatization by hexamethyl disilazane ( HMDS) . 


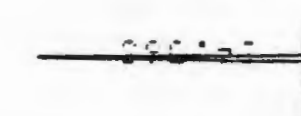

$\ldots$

$+$

2.5
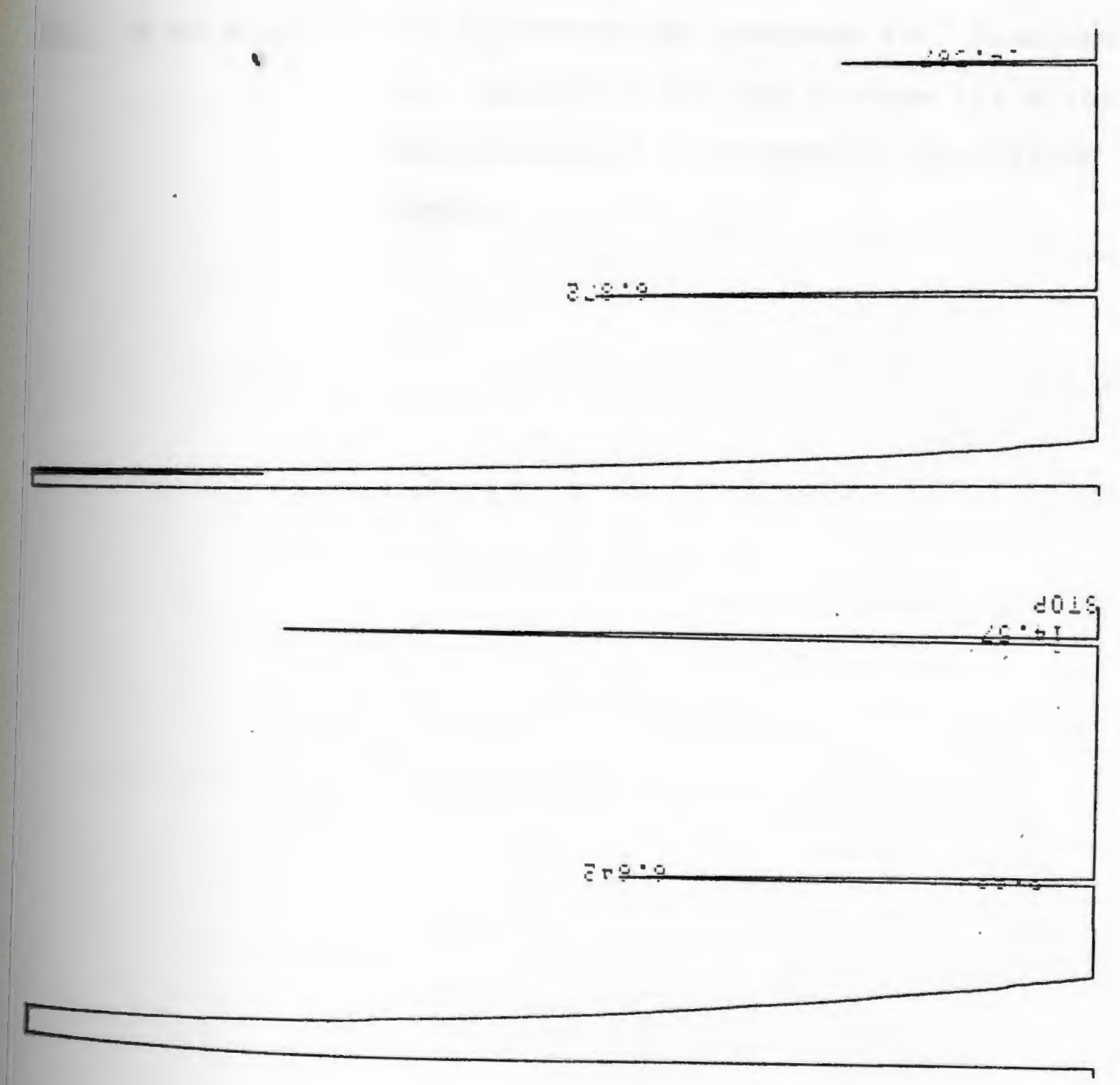

$-170-$ 
Fig. $38 \mathrm{a}, \mathrm{b}$ and $\mathrm{c}$ Gas chromatograms obtained for fructose (a), galactose (b) and glucose (c) after derivatization by hexamethyl disilazane (HMDS) . 


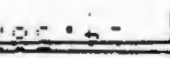
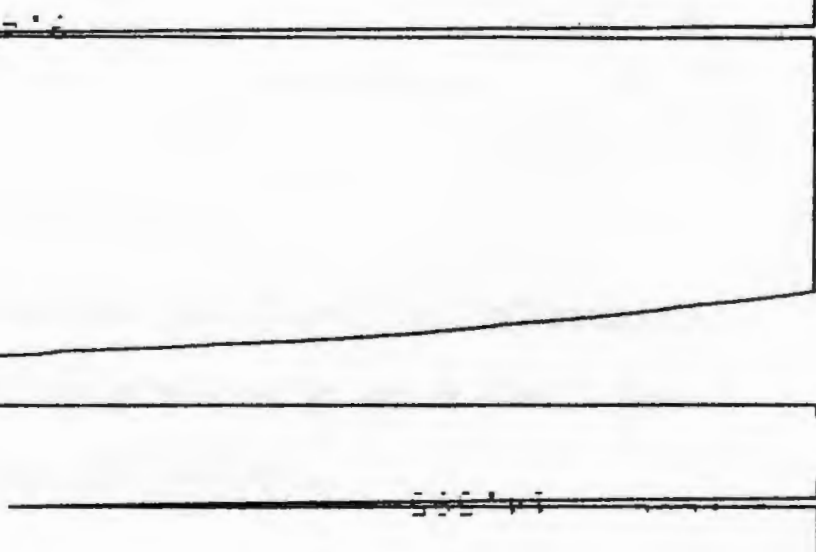

$+\infty$

$-195$

$\therefore .5$ 
Fig. 39 a,b Gas chromatograms obtained for glucuronic acid (a) and galacturonic acid (b) after derivatization by (HMDS).

Fig. 39 c Gas chromatographic profile of the sugars and sugar derivatives which excreted into the culture broth during the fermentation process by Arthrobacter JIZAN-1, after acid hydrolysis and derivatization with HMDS. 
$=0$

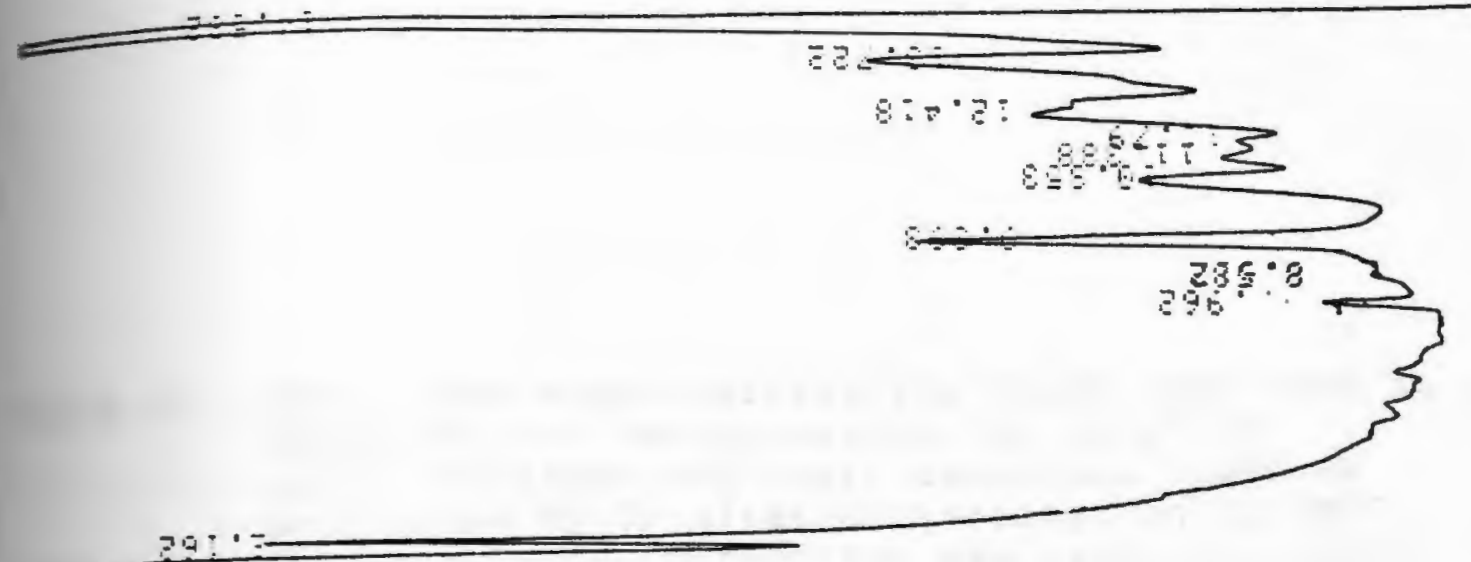

$90^{\circ} \cdot 1$.

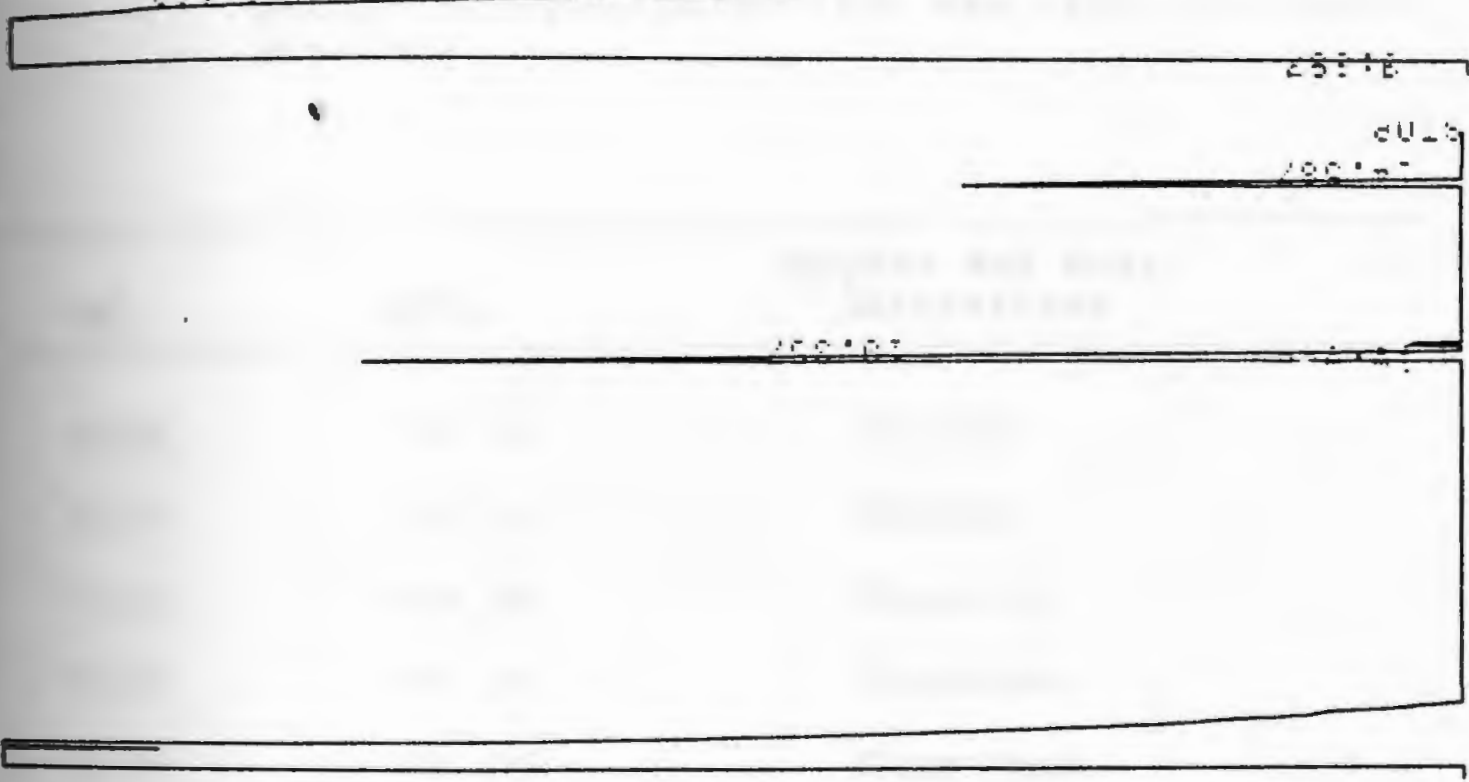

$-1015$

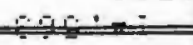


Table 14 Sugars and sugar derivatives which were used as standards for determination the relative retention times and their retention times as determined by GC after derivatization by HMDS. Phenyl-B-D-glucopyranoside was used as internal standard.

\begin{tabular}{lll} 
& Y.H. & $\begin{array}{c}\text { Bugars and sugar } \\
\text { Derivatives }\end{array}$ \\
6.64 & 150.13 & XYlose \\
6.87 & 150.13 & Ribose \\
7.21 & 164.16 & Rhamnose \\
8.59 & 180.16 & Fructose \\
9.24 & 180.16 & Galactose \\
9.43 & 180.16 & Glucose \\
9.53 & 196.16 & Galactonic Acid \\
10.20 & 194.14 & Glucuronic Acid \\
10.36 & 194.14 & galacturonic Acid \\
14.57 & - & Phenyl-B-D- \\
& Glucopyranoside & \\
\hline & &
\end{tabular}


Table 15 Sugar and sugar derivatives obtained from the culture broth by Arthrobacteri JIZAN-1 after acid hydrolysis and derivatization by HMDS and their retention times as determined by GC.

\begin{tabular}{lll} 
RT & K.W. & $\begin{array}{c}\text { Bugars and sugar } \\
\text { Derivatives }\end{array}$ \\
2.18 & - & Unknown \\
7.97 & 164.16 & Rhamnose \\
9.39 & 180.16 & Glucose \\
10.84 & 194.14 & Galacturonic Acid \\
12.43 & - & Unknown \\
13.75 & - & Unknown \\
14.57 & - & Phenyl-B-D- \\
& & Glucopyranoside \\
\hline
\end{tabular}


III. Production of biosurfactant by continuous minentation:

For the bulk production of biosurfactant, a primary prequisite is the definition of culture conditions in which high yields of surface active compounds are obtained by a particular microorganism. This forms the basis for a cealistic estimation of the feasibility of biosurfactant poduction. The aim of such a process is to design a continuous fermentation to produce at the cheapest rate a large amount of biosurfactant by Arthrobacter JIZAN-1 with glucose as the carbon source.

The influences of the nitrogen sources (ammonium chloride and ammonium nitrate) and the amount of carbon source (glucose) on biosurfactant production were investigated by cultivation of JIZAN-1 on BMSM containing $0.02 \%$ yeast extract in a continuous culture with a dilution rate of $0.11 \mathrm{hr}^{-1}$.

A. Influence of ammonium chloride $\left(\mathrm{NH}_{4} \mathrm{Cl}\right)$ as $\mathrm{N}$-source on biosurfactant production by continuous fermentation: The isolated organism JIZAN-1 produced surface active compound when cultivated in batch culture on MMSM containing 0.28 glucose and $0.02 \%$ yeast extract. The minimum values for surface and interfacial tensions were 27.2 and 2.2 dynes/cm respectively, when the organism JIZAN-1 was cultivated in batch culture on BMSM containing $0.15 \% \mathrm{NH}_{4} \mathrm{Cl}$ as $\mathrm{N}$-source, 0.28 glucose as c-source and $0.02 \%$ yeast extract, but when this organism (JIZAN-1) was cultivated in continuous culture 
on the same media and conditions used for batch culture except for $\mathrm{pH}$, the minimum surface and interfacial tensions were 28.1 and 3.0 dynes/cm respectively as shown by Fig. 40 $a, b$. This indicates that the production of biosurfactant was less in continuous culture than batch culture when $\mathrm{NH}_{4} \mathrm{Cl}$ was used as $\mathrm{N}$-source. Continuous fermentation was initiated when the culture reached mid-exponential phase of growth (130 KU), by addition of fresh MMSM containing $0.2 \%$ glucose, $0.02 \%$ yeast extract and $0.15 \% \mathrm{NH}_{4} \mathrm{Cl}$, to the fermentor, and the fermentation process was controlled at $30^{\circ} \mathrm{C}, \mathrm{pH} 7.0$ and the agitation was $200 \mathrm{rpm}$. Foam was controlled by electrical sensing which signaled the addition of antifoam $A$ concentrate solution, which was utilized for foam suppression. Fig. $40 \mathrm{a}, \mathrm{b}$ shows the growth response and the reduction of surface and interfacial tensions verses time in a continuous culture with limited glucose $(0.2 \%)$ and, $\mathrm{NH}_{4} \mathrm{Cl}$ $(0.15 \%)$ as $\mathrm{N}$-source at a dilution rate of $0.11 \mathrm{hr}^{-1}$. The biomass was maintained around $280 \mathrm{KU}$ and the surface and interfacial tensions were respectively 29.0 and 3.0 dynes/cm. The continuous fermentation process (biomass concentration and surface activity) was followed for $25 \mathrm{hrs}$. Residual ammonium chloride $\left(\mathrm{NH}_{4} \mathrm{Cl}\right)$ was $240 \mu \mathrm{g} / \mathrm{ml}$, residual glucose was $80 \mu \mathrm{g} / \mathrm{ml}$ and the biomass was $0.91 \mathrm{~g} / \mathrm{L}$. 
Fig. 40 a, b、 Surface (a) and interfacial tensions (b) of a whole culture broth of Arthrobacter JIZAN-1 as related to its growth in continuous culture on filter sterilized BMSM containing $0.2 \%$ glucose, $0.15 \% \mathrm{NH}_{4} \mathrm{Cl}$ and $0.02 \%$ yeast extract at $200 \mathrm{rpm}, 30^{\circ} \mathrm{C}, \mathrm{pH} 7.0$ and 0.11 $\mathrm{hr}^{-1}$ dilution rate. 

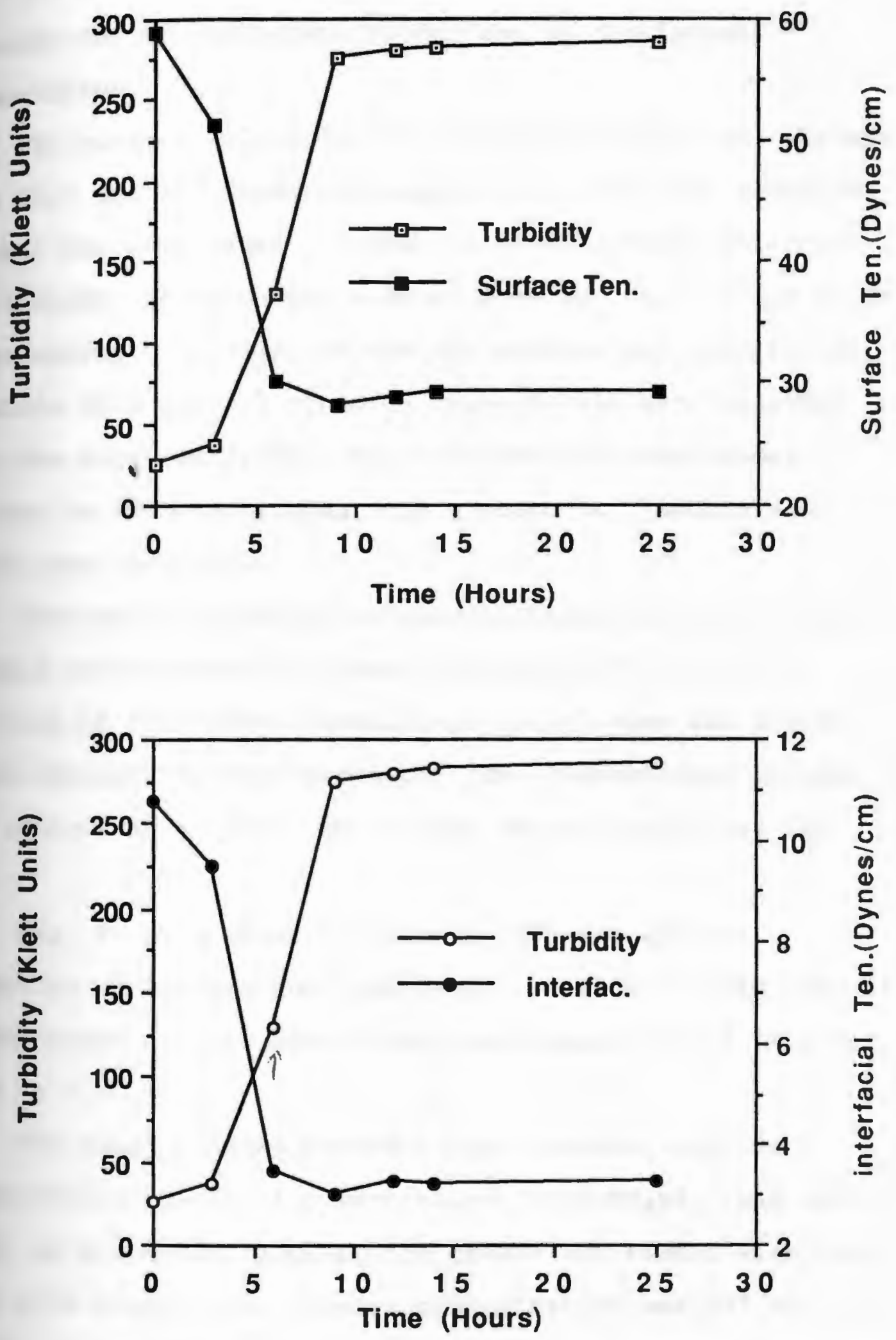
B. Influence of ammonium nitrate $\left(\mathrm{NH}_{4} \mathrm{NO}_{3}\right)$ as the i-source for biosurfactant production by continuous cermentation:

The minimum values for surface and interfacial tensions were 26.5 and 2.0 dynes/cm respectively, when the organism JIZAN-1 was cultivated in batch culture on BMSM containing $0.158 \mathrm{NH}_{4} \mathrm{NO}_{3}$ as $\mathrm{N}$-source, $0.2 \%$ glucose as C-source and $0.02 \%$ yeast extract. Similar values for surface and interfacial tensions 26.8 and 1.9 dynes/cm respectively were obtained when the orgąnism JIZAN-1 was cultivated in continuous culture on MMSM containing $0.3 \%$ glucose as C-source and 0.028 yeast extract.

Continuous fermentation was initiated when the culture reached mid-exponential phase of growth (175 KU), by addition of fresh MMSM containing $0.3 \%$ glucose and $0.02 \%$ yeast extract, to the fermentor. The fermentation process was controlled at $30^{\circ} \mathrm{C}, \mathrm{pH} 7.0$ and the agitation was 200 rpm.

Fig. $41 \mathrm{a}, \mathrm{b}$ shows the growth response and the reduction of surface and interfacial tensions verses time in a continuous culture with $\mathrm{NH}_{4} \mathrm{NO}_{3}$ as $\mathrm{N}$-source and a dilution rate of $0.11 \mathrm{hr}^{-1}$.

The final biomass concentration (growth) and the biosurfactant production were higher with $\mathrm{NH}_{4} \mathrm{NO}_{3}$ than with $\mathrm{NH}_{4} \mathrm{Cl}$ as $\mathrm{N}$-sources, however the growth was faster with $\mathrm{NH}_{4} \mathrm{Cl}$ than with $\mathrm{NH}_{4} \mathrm{NO}_{3}$ (the biomass concentration was $275 \mathrm{KU}$ after 9 hrs. of cultivation on $\mathrm{NH}_{4} \mathrm{CI}$ as $\mathrm{N}$-source, whereas the 
Fig. 41 a, b Surface (a) and interfacial tensions (b) of a - whole culture broth of Arthrobacter JIZAN-1 as related to its growth in continous culture of filter sterilized BMSM containing $0.3 \%$ glucose, $0.15 \% \mathrm{NH}_{4} \mathrm{NO}_{3}$ and $0.02 \%$ yeast extract at $200 \mathrm{rpm}, 30^{\circ} \mathrm{C}, \mathrm{pH} 7.0$ and $0.11 \mathrm{hr}^{-1}$ dilution rate. 

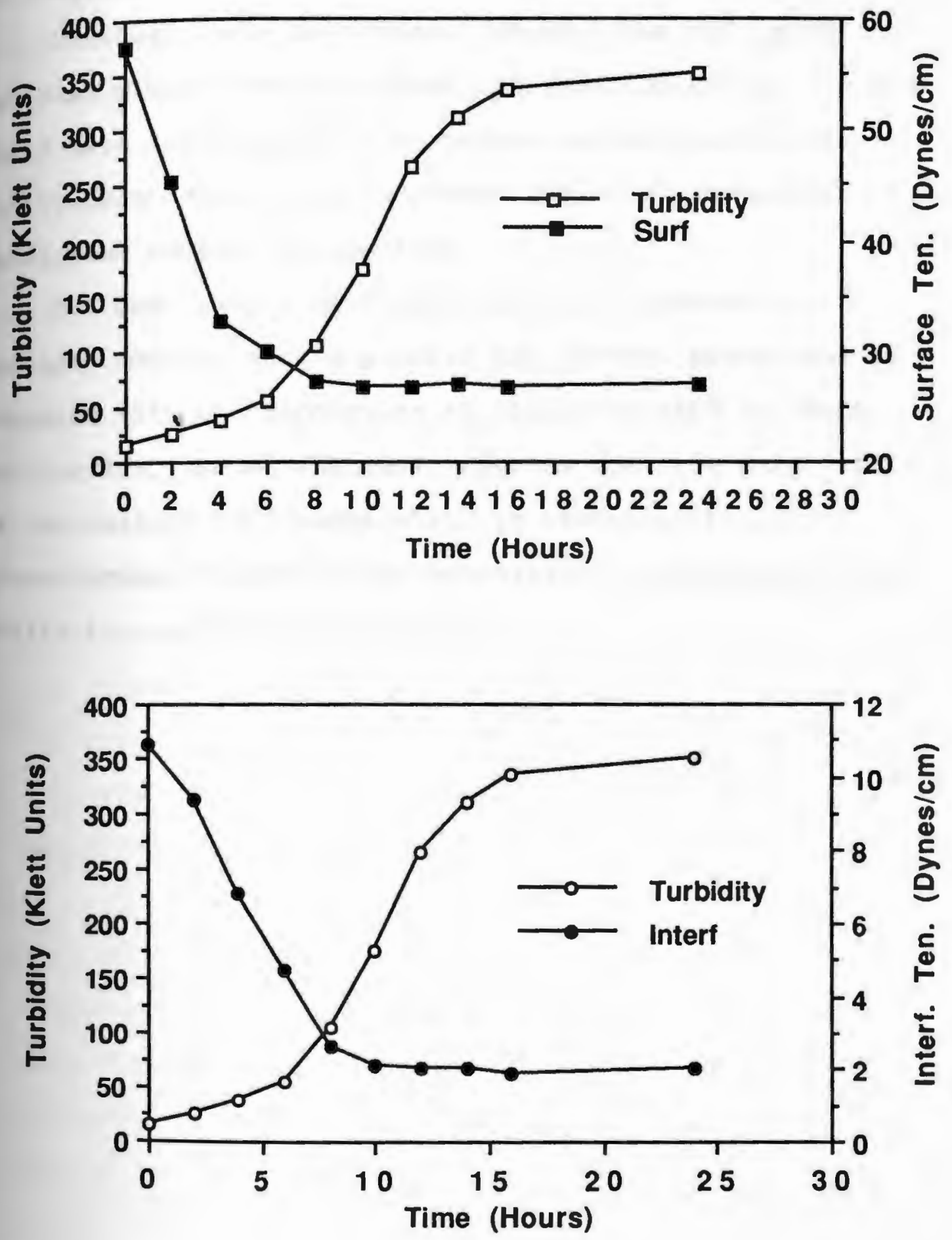
biomass concentration was 265 after $12 \mathrm{hrs}$. of cultivation on $\left(\mathrm{NH}_{4} \mathrm{NO}_{3}\right)$ as shown by Fig. 38 and Fig. 41.

Residual ammonium nitrate $\left(\mathrm{NH}_{4} \mathrm{NO}_{3}\right)$ was $520 \mu \mathrm{g} / \mathrm{ml}$, mesidual glucose was $320 \mu \mathrm{g} / \mathrm{ml}$ and the biomass was $1.2 \mathrm{~g} / \mathrm{L}$. These data indicates that by proper medium design and cultivation conditions the production of biosurfactant in montinuous culture is possible.

The goal was to develop a continuous production process, because such a process has several advantages as compared with the production of biosurfactants in batch cultivation, one of the advantages is that the exact control of the culture conditions which is essential for high dosurfactant formation by the cells is accomplished more easily in a continuous culture. 
IV. Isolation and purification of the biosurfactant:

There are many methods applicable to the isolation of psurfactants. The method used for the isolation of a ertain product depends on the nature of this compound (whether it is water-soluble or not, anionic or nonionic, cell wall bound or extracellular). Therefore there are no general rules for the isolation of biosurfactant. A suitable method or a combination of suitable methods for product recovery has to be developed for every new isolated compound (Kasaric et al., 1987).

A. Extraction of the biosurfactant:

Biosurfactant produced by Arthrobacter JIZAN-1 is ter-soluble extracellular surfactant. Different organic solvents such as chloroform, ethyl acetate, petroleum ether, hexane, benzene, and various combination of each two solvents at different ratios and temperatures were used to extract the biosurfactant from the cell-free culture broth. Multiple extractions using a separatory funnel were used to recover the biosurfactant from the culture broth. The ability of the organic solvent to extract the biosurfactant was determined by measuring the surface tension of the culture broth after extraction and the extract from which the organic solvent had been removed and the residue dissolved in distilled water.

Fig. 42 shows the surface and interfacial tensions of the control (Dist. $\mathrm{H}_{2} \mathrm{O}$ ), the culture broth before and after extraction with chloroform at $35^{\circ} \mathrm{C}$ and the chloroform 
Fig. 42 Surface and interfacial tensions obtained from Arthrobacter JIZAN-1 culture broth before extraction, after extraction with chloroform at $35^{\circ} \mathrm{C}$ and the chloroform extract after evaporation of the chloroform and redissolving in deionized $\mathrm{H}_{2} \mathrm{O}$ as determined by Fisher autotensiomat.

1-Control (Dist.Water)

2-Culture Broth Before Extraction

3-Culture Broth After Extraction At 35 C

4-Chloroform Extract

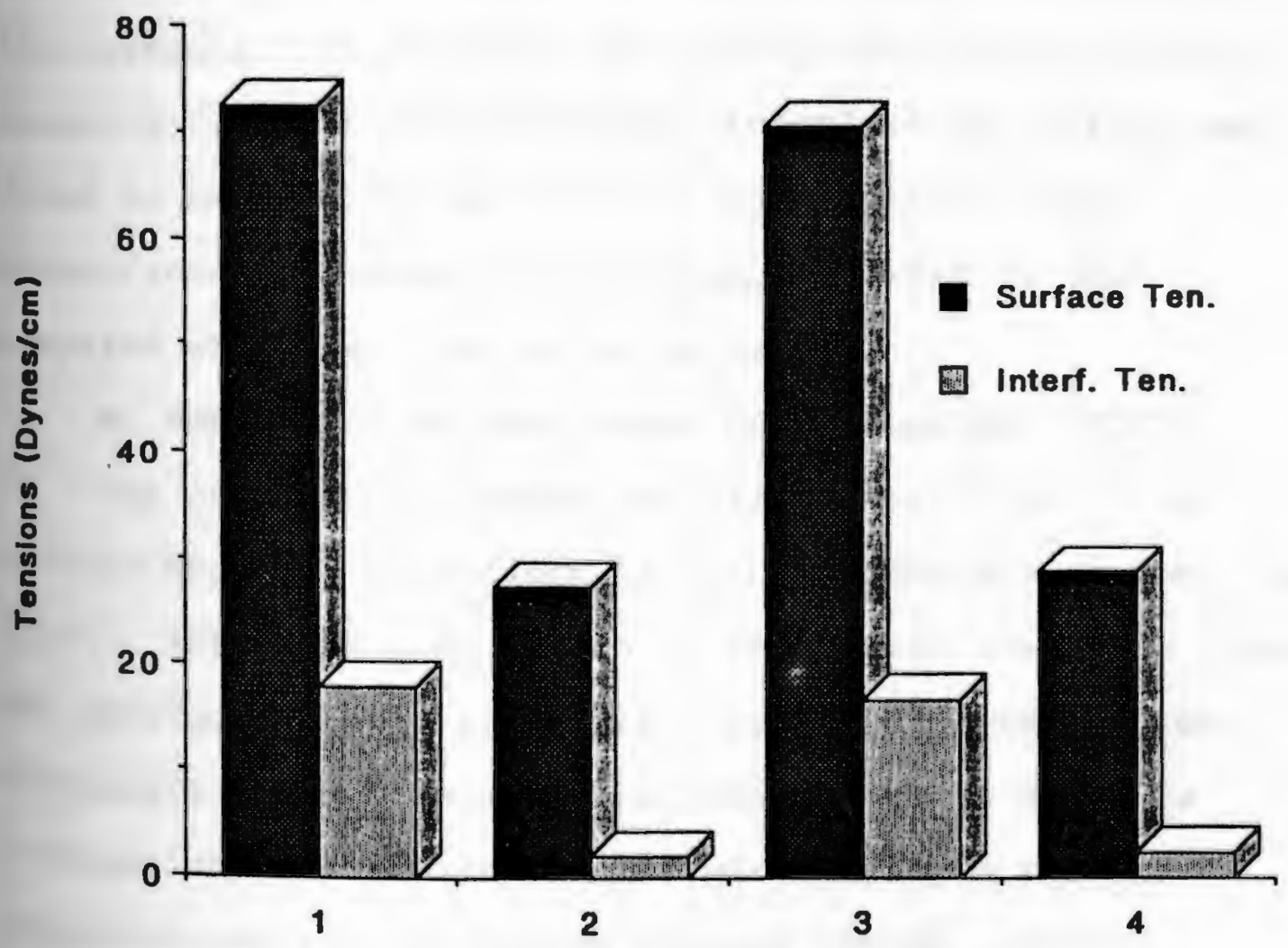


extract after the complete evaporation of chloroform and the residue dissolved in the same volume of deionized $\mathrm{H}_{2} \mathrm{O}$. The surface and interfacial tension values for the control (Dist. $\mathrm{H}_{2} \mathrm{O}$ ) were 72.3 and 18 dynes/cm, the culture broth before extraction were 27.2 and 2.0 dynes $/ \mathrm{cm}$, the culture broth after extraction with chloroform at $35^{\circ} \mathrm{C}$ were 70.1 and 16.8 dynes/cm, and the chloroform extract after evaporation the chloroform and redissolving in dist. $\mathrm{H}_{2} \mathrm{O}$ were 28.7 and 2.5 dynes/cm respectively.

The data obtained from Fig. 42 shows that more than $95 \%$ of the biosurfactant was extracted from the culture broth by chloroform after shifting the culture broth temperature to $35^{\circ} \mathrm{C}$, and also indicates that the organic solvent chloroform did not alter the surfactant properties but it did remove the surfactant which cause the low surface and interfacial tensions from the culture broth, therefore chloroform was found to be the best solvent for extraction of the biosurfactant produced by Arthrobacter JIZAN-1, when compared with the other solvents used.

\section{B. Separation by thin layer chromatography (TLC):}

The extract obtained by multiple extraction of the culture broth by chloroform at $35^{\circ} \mathrm{C}$ was designated the crude lipid. After the evaporation of chloroform the crude lipid was spotted on to a silica gel G type TLC plates as the tationary phase. Several HPLC grade organic solvents combinations, as mobile phases, were employed for the separation of the chloroform extract (crude lipid). 
etection reagents such as $0.2 \%$ anthrone-sulfuric acid, lodine vapours, and UV light were used for visualizing mompounds on TLC plate.

The results of these separations indicated that, hloroform, ethyl acetate and methanol $(50: 30: 20)$ as the mobile phase, the crude extract was separated into four bands, as detected by UV light, and iodine vapours, but only three bands were detected by anthrone-sulfuric acid. One band stayed on the base line, two bands moved to the middle of the TLC plate with poor resolution and the fourth band moved up with the solvent front. The four bands were detected on the silica gel $G$ plate without fluorescent indicator by UV light, which indicated that these compounds were fluorescence. The four bands were detected by iodine vapours, which indicates the presence of unsaturated carbon to carbon bonds in these compounds. The positive reactivity of three bands with anthrone reagent suggests that one of the constituents of these compounds is carbohydrate.

For a better separation (resolution) of the two bands which moved into the middle of the TLC plate, different HPLC grade organic solvent combinations were used as mobile phases for developing the TLC plate. One mobile phase hexane, chloroform, ethyl acetate and methanol (30:40:20:10) was found to give the best separation (resolution) for those two bands on silica gel G type TLC plates. 


\section{c. Purification by column chromatography:}

The TLC separation of the chloroform extract (crude 11pid) was used as a primary step followed by purification of the biosurfactant by column chromatography.

A flash-chromatography column equipped with threaded joints and a solvent reservior was packed with silica gel absorbent (100-200 mesh size) as the stationary phase. Two mobile phases, one contains hexane, chloroform, ethyl acetate and methanol $(30: 40: 20: 10)$ and the other mobile phase was HPLC grade methanol, were used for purification of the biosurfactant.

A fraction collector was used to collect $7 \mathrm{ml}$ fractions, and each fraction or a group of fractions were concentrated by evaporation of the organic solvents using a rotary evaporator, tested for the purity by TLC, redissolved in deionized water and examined for surface activity with the Fisher autotensiomat. As a result, two bands were separated and followed by UV light during the elution with the first mobile phase hexane, chloroform ethyl acetate and methanol (30:40:20:10), and examined for the purity by TLC plate. The first band eluted from the column moved up with the solvent front on the TLC plate, whereas the second band eluted from the column was separated into two spots in the middle of the TLC plate. The surface activity of each band was tested, and there was no surface activity found to be associated with the components of the two bands. 
The third band which was trapped in the column and did not move with the first mobile phase as detected by UV light, was eluted with HPLC grade pure methanol as a mobile phase. Surface activity of the methanol eluent was tested, and the surface and interfacial tension values were respectively 28.2 and 2.7 dynes/cm. Therefore, this test indicates that the surface activity was associated with methanol eluate. The purity of the methanol eluate was examined by spotting $15 \mu \mathrm{l}$ onto a TLC plate, which was then developed by hexane, chloroform, ethyl acetate and methanol (30:40:20:10) as a mobile phase. Only one band was detected on the base line of the TLC plate by using either UV light, iodine vapours or anthrone-sulfuric acid.

Methanol was evaporated at $40^{\circ} \mathrm{C}$ by vacuum evaporator, then the residue was redissolved in dist. $\mathrm{H}_{2} \mathrm{O}$ and freeze dried. The freeze dried powder was designated surfactant $\# 1$.

The correlation between surfactant \#1 concentration and the reduction of surface activity:

Surfactant \#1 in the range of $0.2-2.0 \mathrm{mg} / 100 \mathrm{ml}$ of water with $0.2 \mathrm{mg} / 100 \mathrm{ml}$ increment was used to determine the relationship between the surfactant \#1 concentration and the reduction of surface and interfacial tension. The surface and interfacial tensions decreased with the increase of surfactant \#1 concentration to concentration of $1.6 \mathrm{mg} / 100$ ml. Any further increases in concentration beyond this point did not result in a substantial reduction of surface 
Fig. $43 \mathrm{a}, \mathrm{b}$. The correlation between surfactant \#1 concentration and surface activity (decreasing of surface and interfacial tensions with increasing surfactnat \#1 concentration) as determined by Fisher autotensiomat. 

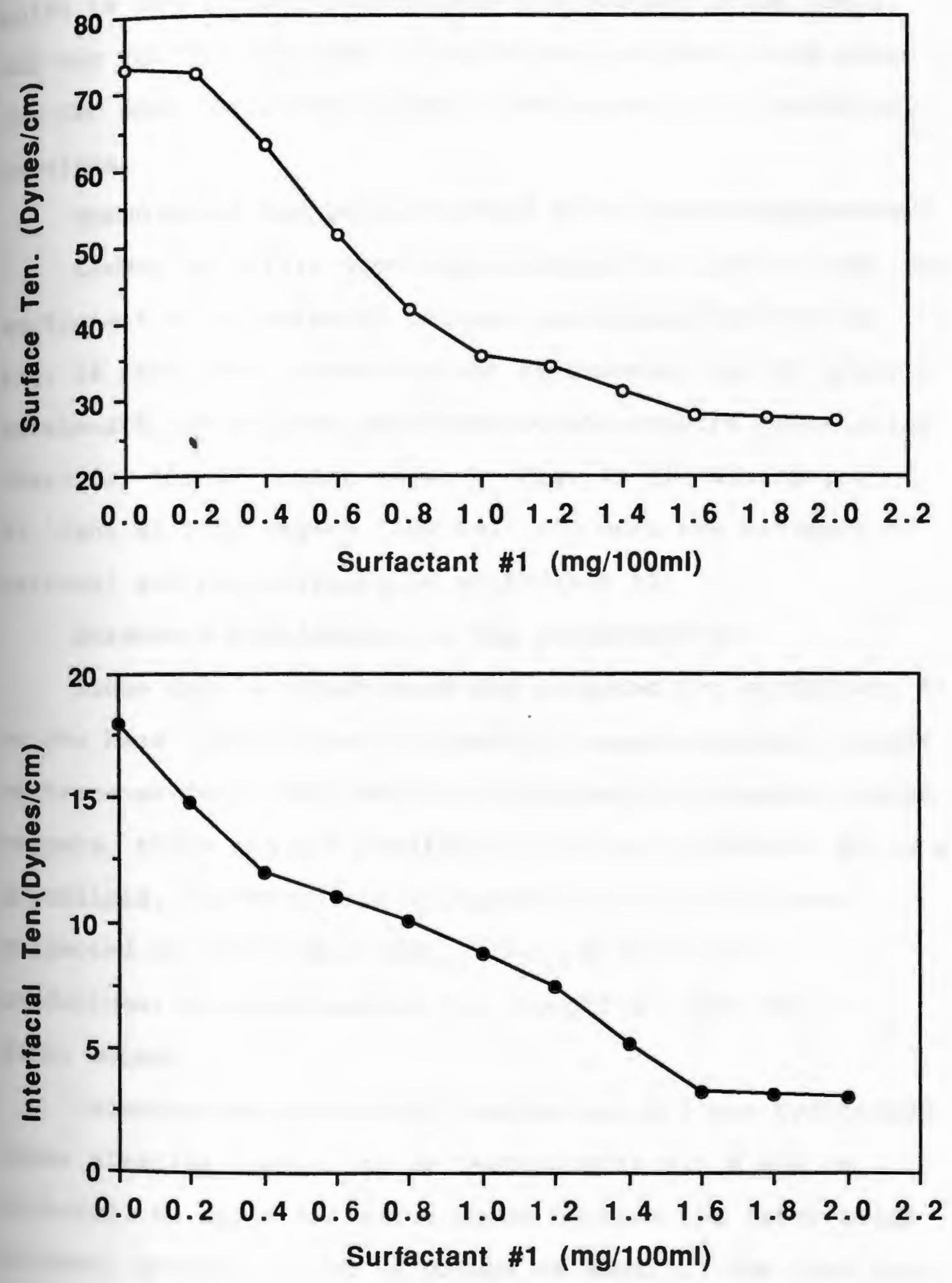
or interfacial tensions as shown by Fig. $43 \mathrm{a}, \mathrm{b},$. This point is called the critical micelle concentration (CMC), and any further increase in surfactant concentration above the CMC does not normally alter the surface or interfacial tensions.

Scanning of the biosurfactant \#1 by spectrophotometer:

Lambda 4B UV/VIS spectrophotometer was used to scan the surfactant \#1 in methanol between wavelength 900-190 nm. Fig. 44 shows that biosurfactant \#1 absorbed the UV light at wavelength $209 \mathrm{~nm}$, and according to the spectra correlation chart for the UV region shown by Fig. 45 the absorption of UV light at this region $(209 \mathrm{~nm})$ indicates the presence of carboxyl and ester groups on surfactant \#1.

Structure elucidation of the surfactant \#1:

Since only a single band was detected for surfactant \#1 on the base line of the TLC plate by sugar specific reagent anthrone-sulfuric acid and by lipid specific reagent iodine vapours, these results confirmed that the surfactant \#1 is a glycolipid, therefore the anthrone positive lipid was subjected to hydrolysis under alkaline and acidic conditions, to characterize the lipophilic part and the sugar moiety.

Anthrone positive lipid (surfactant \#1) was hydrolyzed under alkaline conditions by heating with $0.5 \mathrm{~N} \mathrm{KOH}$ in methanol, to split the ester bonds between the fatty acids carboxyl groups and the $\mathrm{OH}$ groups of sugars. The free fatty acids released were extracted with ether from 
Eig. 44 Surfactant \#1 spectra, which was abtained by scanning between $2 \max 900-190 \mathrm{~nm}$ using Lambda 4B UV/VIS spectrophotometer.

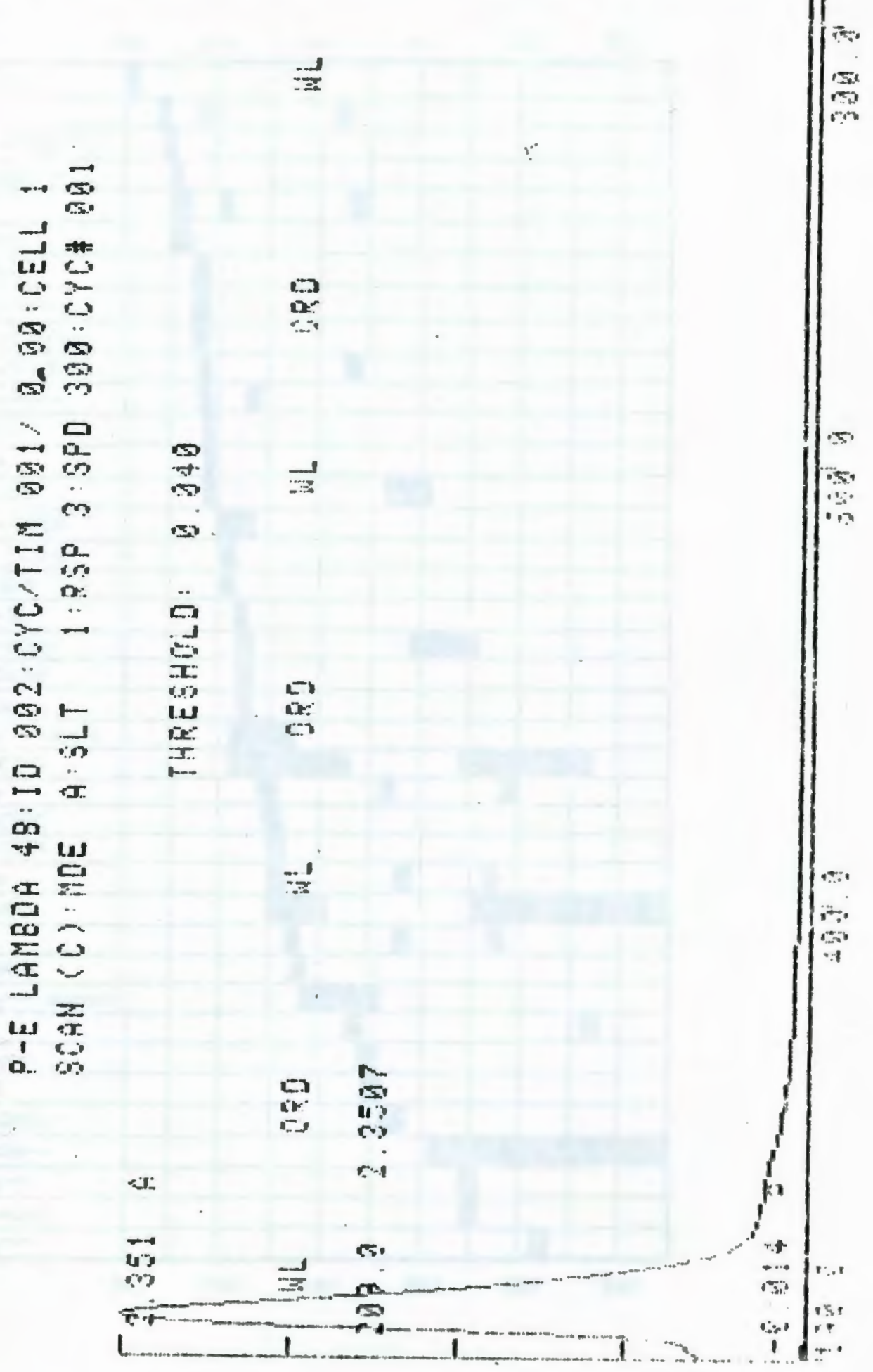


Fig. 45 Spectra correlation chart for the UV region.

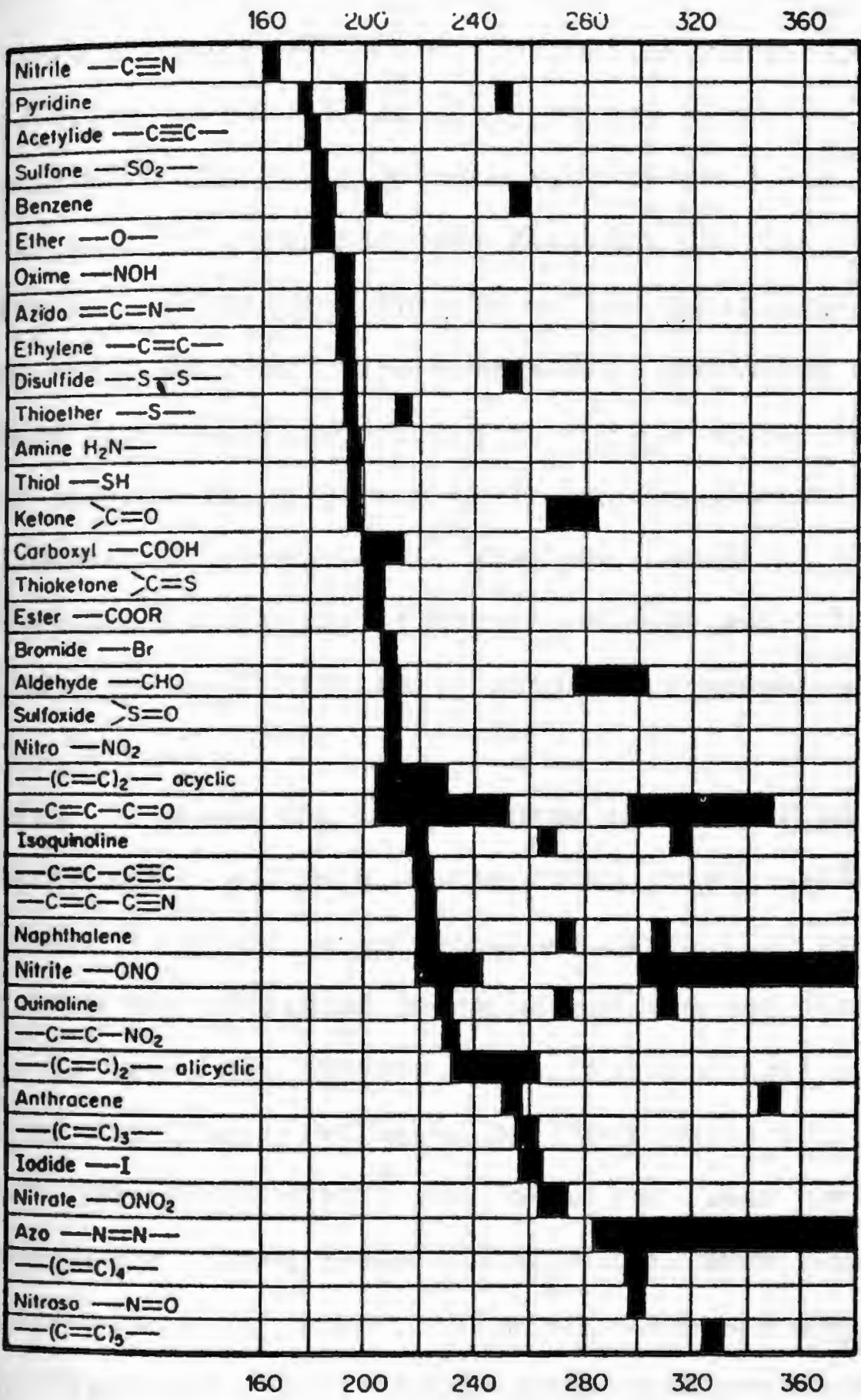


the acidified reaction mixture, then methylated by $\left(\mathrm{BF}_{3}\right)$ in methanol, and the resultant fatty acid methyl esters were letermined by gas chromatography using a flame ionization detector and $10 \%$ sp-2330 (Cyano silicone) column.

Several fatty acid methyl ester standards including $C_{10: 0}, C_{12}: 0$, GLC-10;20;40;50;60;90 and PUFA-1 were used for determination the relative retention times. Fig. 46 a,b,c; Fig. 47 a,b; Fig. 48; Fig. 49; Fig. 50; and Fig. 51 shows the gas chromatographic profile of the fatty acid methyl esters obtained from those standards. Retention times obtained from analyzing of $\mathrm{C}_{10}: 0, \mathrm{C}_{12}: 0$, GLC-10, GLC-20, GLC- 40 and GLC-50 by GC and their correspondence fatty acids were summarized in Table 16, also the retention times obtained from analyzing of GLC- 60 , GLC-90 and PUFA-1 by GC and their corresponding fatty acids were summarized in Table 17.

Fig. 52 shows the chromatogram obtained from the gas chromatographic analysis of the fatty acids released from surfactant \#1 after saponification, extraction by petroleum ether from the acidified reaction mixture and derivatization by $\mathrm{BF}_{3}$ in methanol. Eleven peaks (fatty acids) were detected and identified based on their retention times through comparison with those retention times obtained from the standards. Five peaks (fatty acids) were found to be

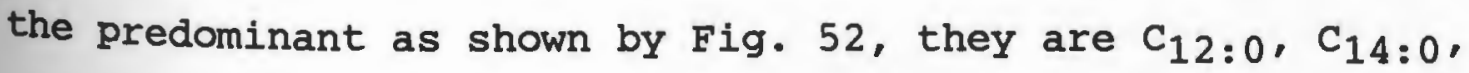
$c_{16: 0}, c_{18: 0}$ and $c_{18: I}$. Fatty acids obtained from surfactant \#1 after saponification (alkaline hydrolysis) and 
Fig. $46 \mathrm{a}, \mathrm{b}$ and $\mathrm{c}$ Gas chromatograms of the fatty acids methyl esters of $C_{10: 0,} C_{12: 0}$ and GLC-10 standards which were used for determination of the relative retention times. 

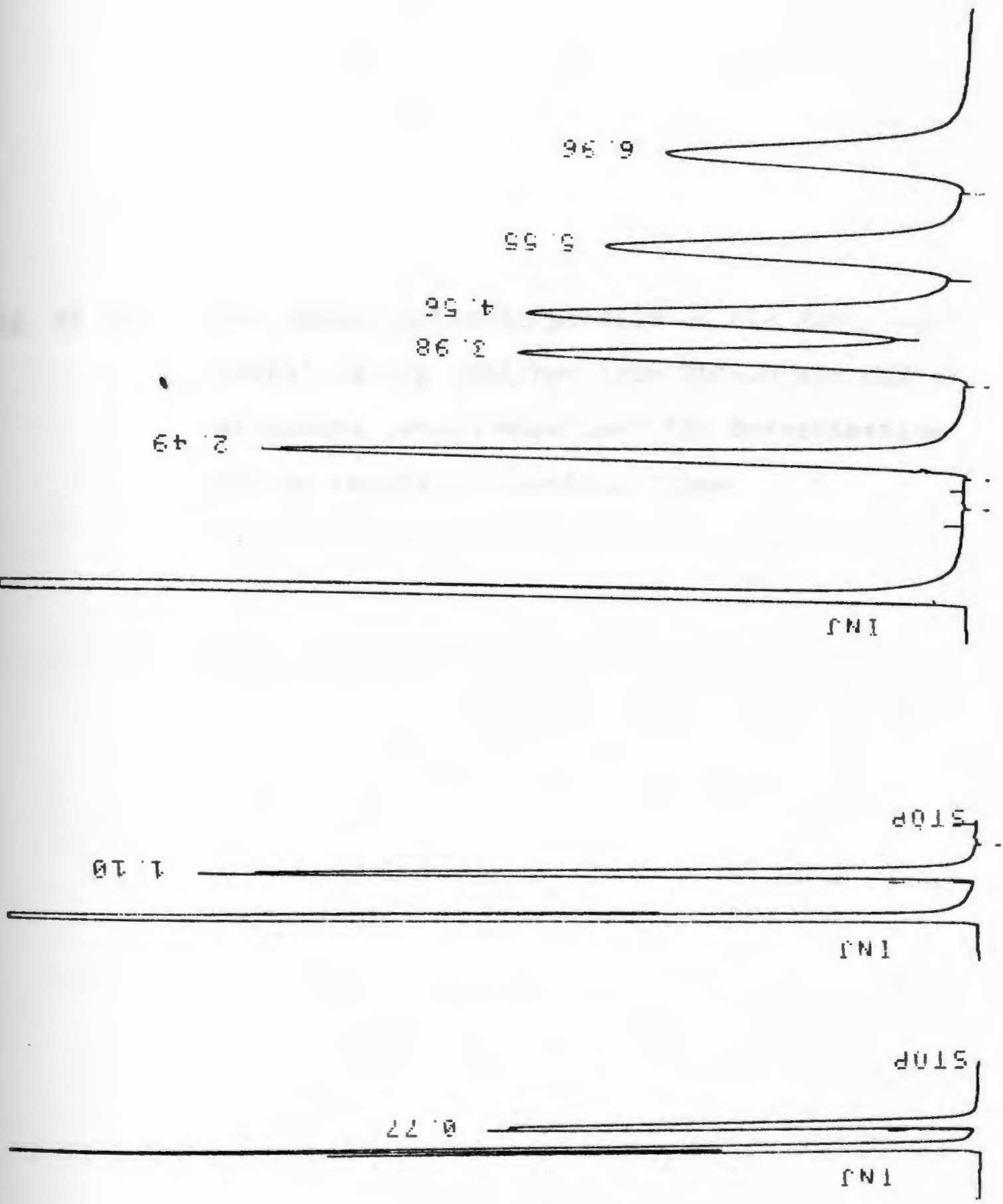
Fig. $47 \mathrm{a}, \mathrm{b}$ Gas chromatographic profile of the fatty acid - methyl esters obtained from GLC-20 and GLC-40 standards, which were used for determination of the relative retention times. 

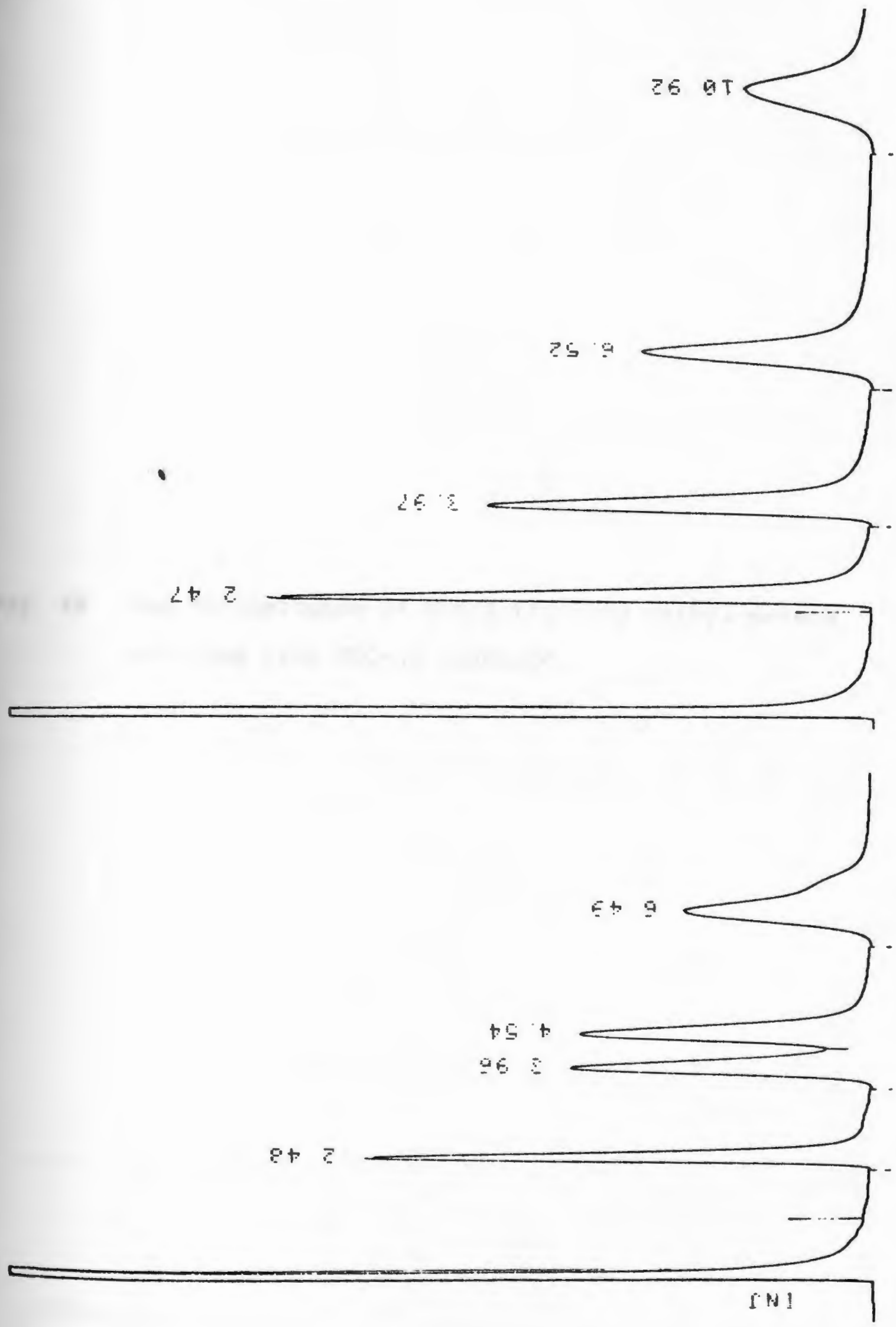

$-200-$ 
Fig. 48 Gas chromatogram of the fatty acid methyl esters obtained from GLC-50 standard. 


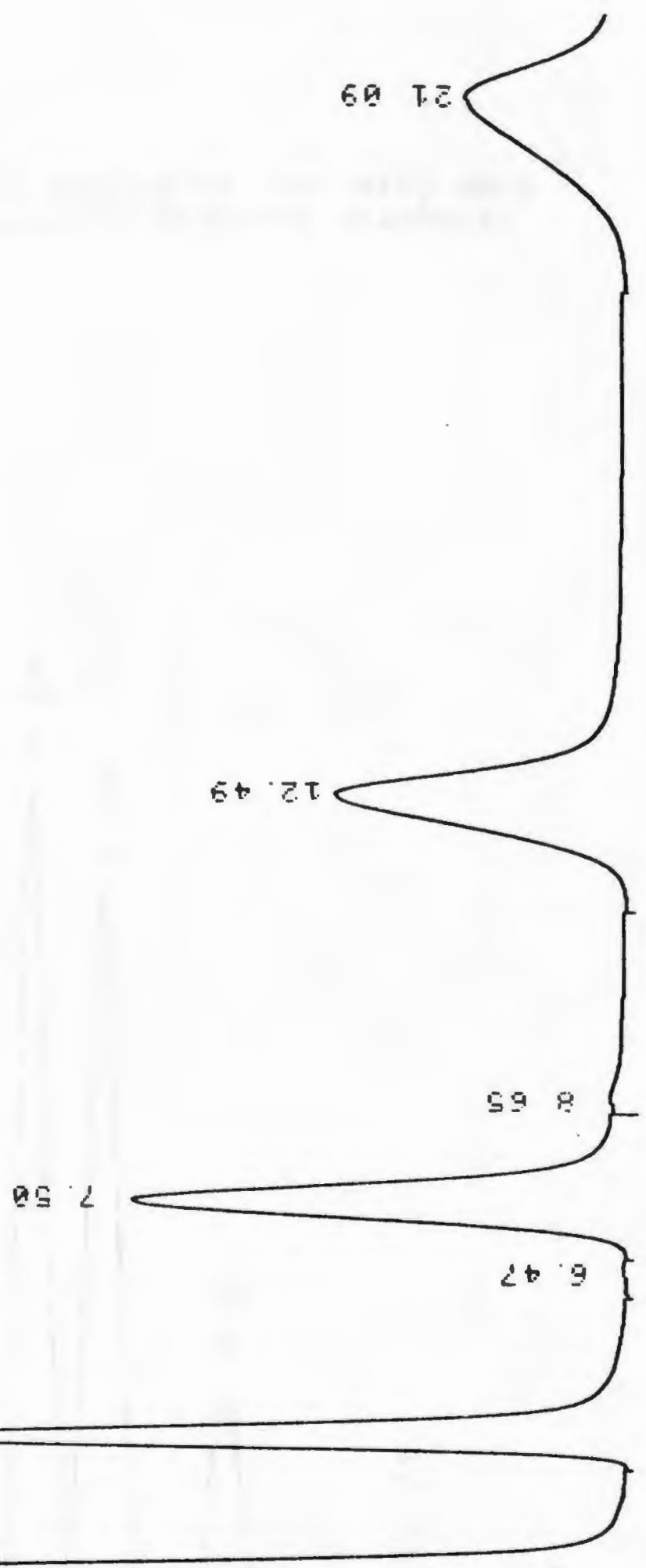

65

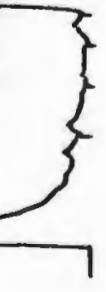


Fig. 49 Gas chromatographic profile of the fatty acid methyl esters obtained from GLC-60 standard.

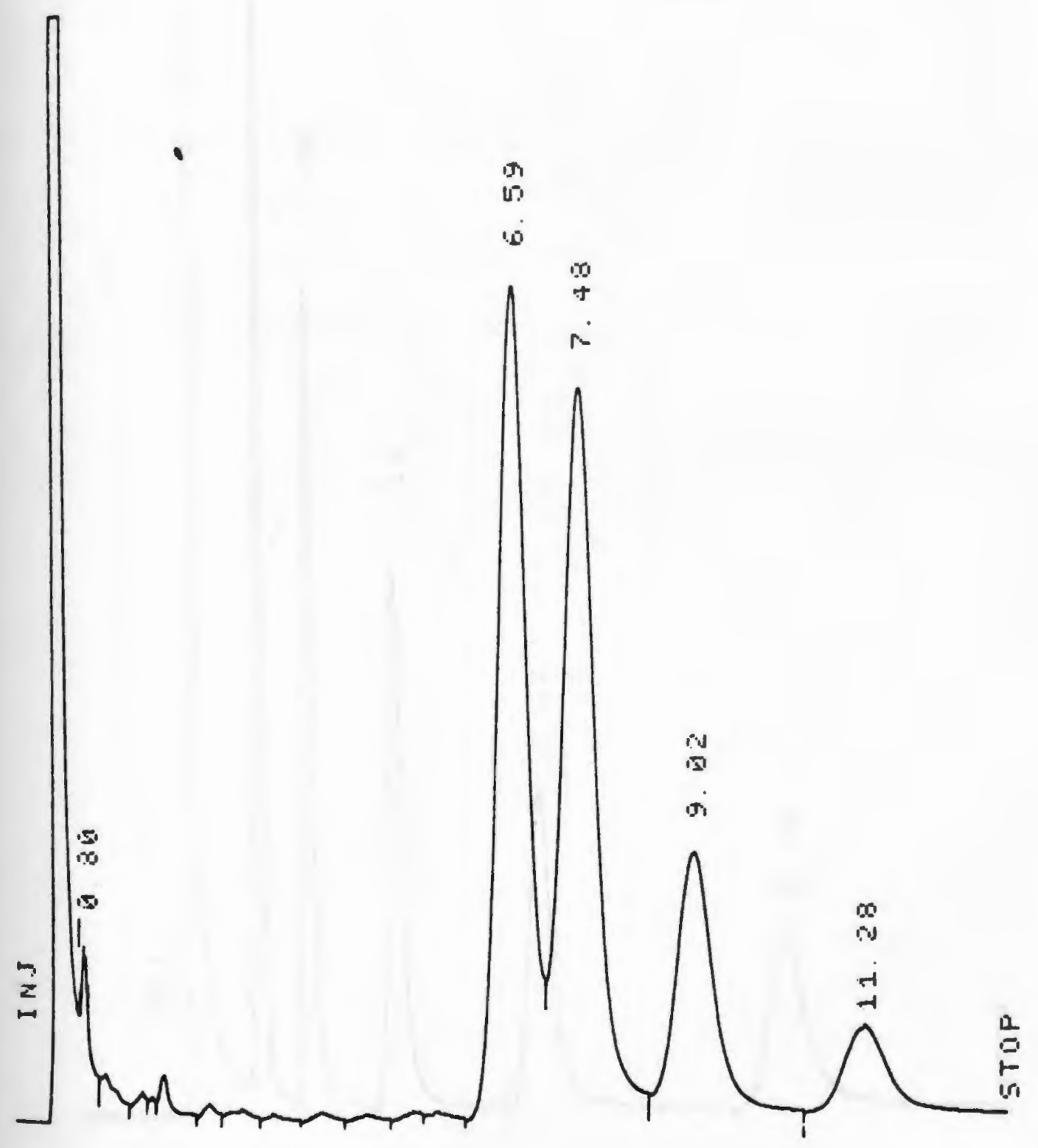


Fig. 50 Gas chromatogram of the fatty acid methyl esters obtained fro GLC-90 standard.

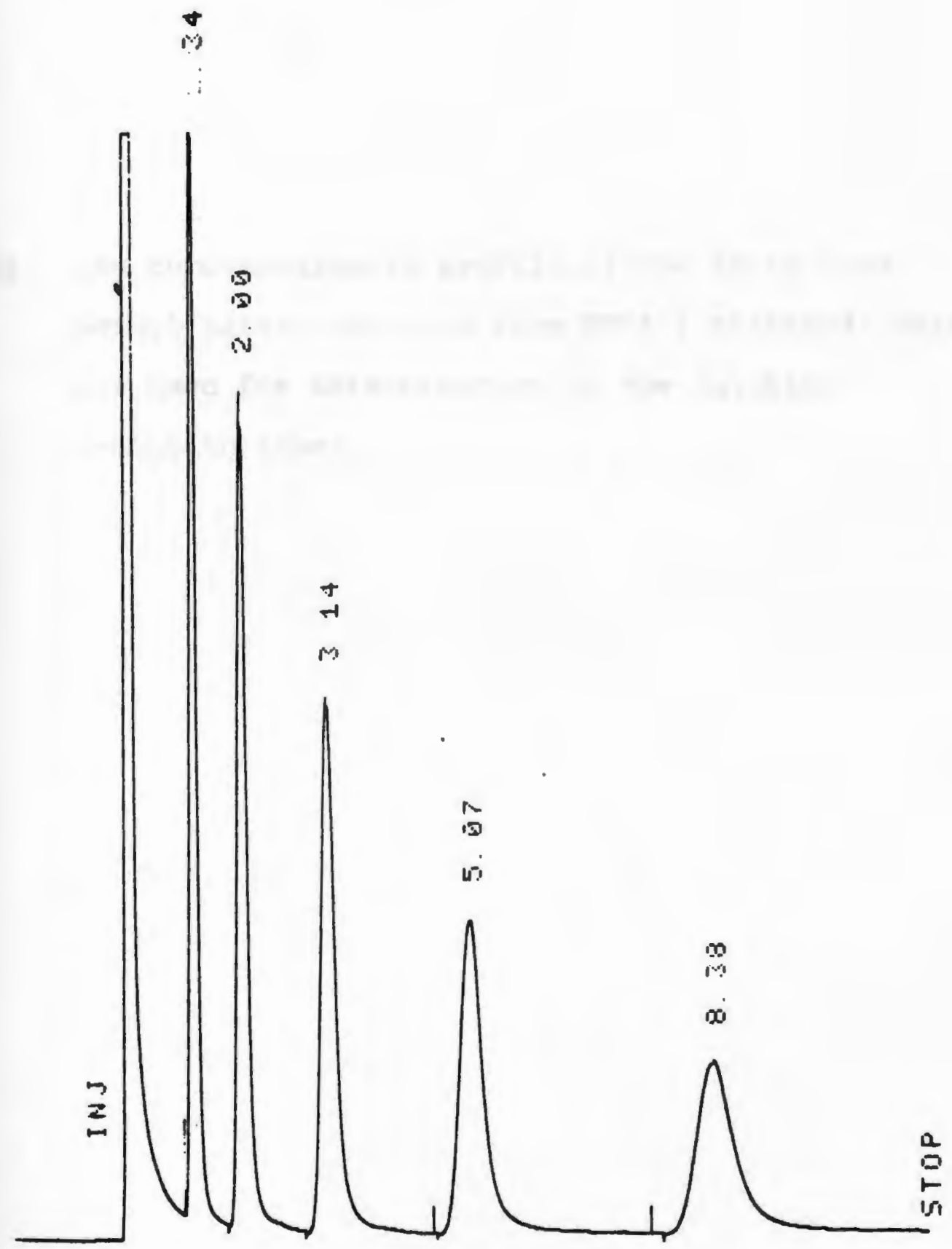


Fig. 51 Gas chromatographic profile of the fatty acid methyl esters obtained from PUFA-1 standard, which was used for determination of the relative retention times. 


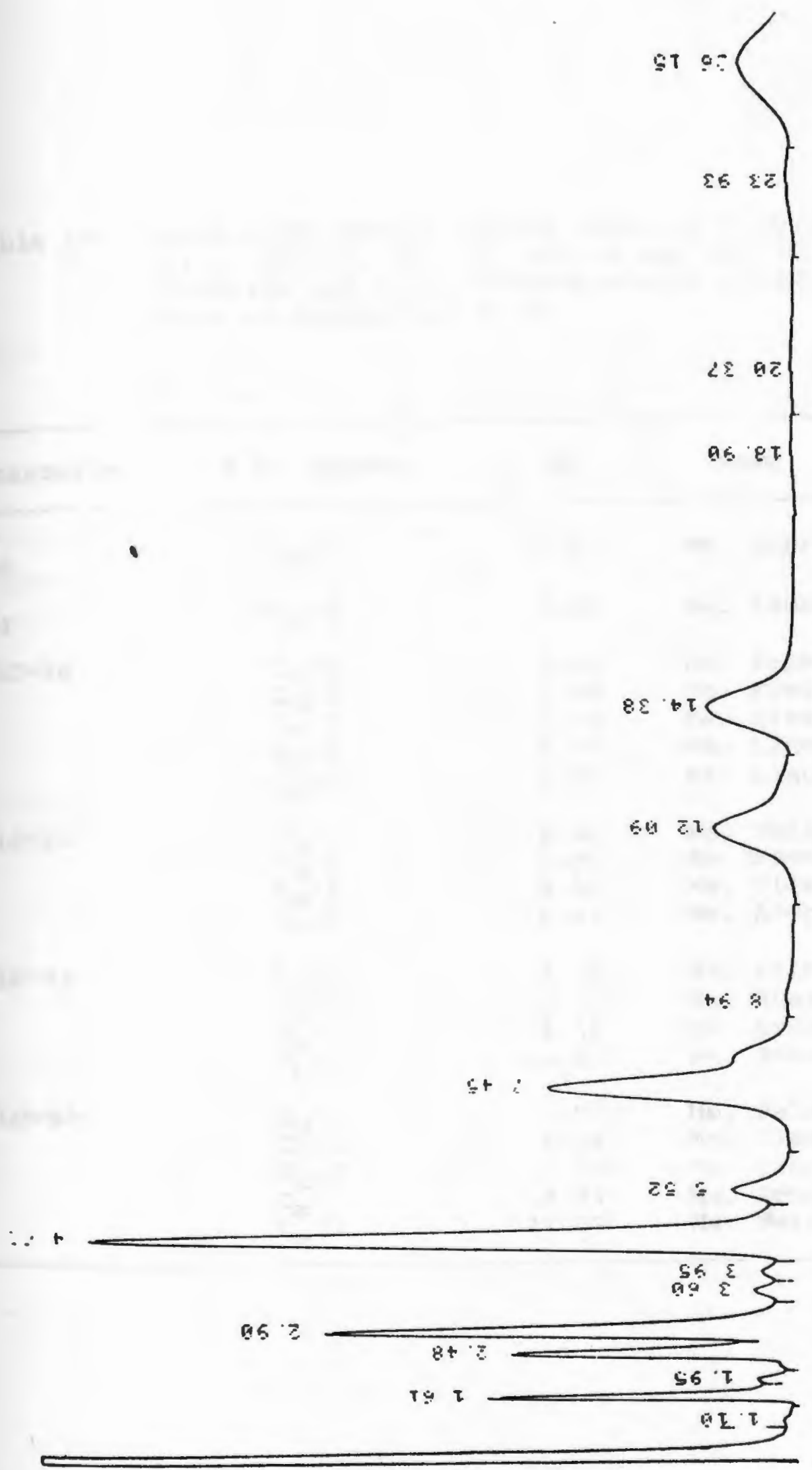

$-206-$ 
Table 16 Fatty acids methyl esters obtained from $\mathrm{C}_{10: 0}$, $C_{12}: 0$, GLC-10, GLC-20, GLC-40 and GLC-50 standards and their correspondence retention times as determined by GC.

\begin{tabular}{llrl} 
standards & * of Carbons & RT & Name \\
\hline$C_{10}$ & $C_{10}: 0$ & 0.81 & Me. Caprate \\
$C_{12}$ & $C_{12}: 0$ & 1.10 & Me. Laurate \\
GLC-10 & $C_{16}: 0$ & 2.49 & Me. Palmitate \\
& $C_{18}: 0$ & 3.98 & Me. Stearate \\
& $C_{18}: 1$ & 4.56 & Me. Oleate \\
& $C_{18}: 2$ & 5.55 & Me. Linoleate \\
GLC-20 & $C_{18}: 3$ & 6.96 & Me. Linolenate \\
& $C_{16}: 0$ & 2.48 & Me. Palmitate \\
& $C_{18}: 0$ & 3.96 & Me. Stearate \\
& $C_{18}: 1$ & 4.54 & Me. Oleate \\
GLC-40 & $C_{20}: 0$ & 6.49 & Me. Arachidate \\
& $C_{18}: 0$ & 2.47 & Me. Palmitate \\
& $C_{18}: 0$ & 3.97 & Me. Stearate \\
& $C_{20}: 0$ & 6.52 & Me. Arachidate \\
& $C_{22}: 0$ & 10.92 & Me. Beherate \\
GLC-50 & $C_{18}: 1$ & 2.92 & Me. Palmitoeate \\
& $C_{18}: 1$ & 4.59 & Me. Oleate \\
& $C_{20}: 1$ & 12.50 & Me. Eicosenoate \\
& $C_{22}: 1$ & 21.09 & Me. Erucate \\
& $C_{24}: 1$ & & \\
& & &
\end{tabular}


Table 17 Fatty acid methy esters obtained from GLC-60, GLC-90 and PURA-1 standards and their correspondence retention times as determined by GC.

\begin{tabular}{|c|c|c|c|c|}
\hline standards & * of & Carbons & RT & Name \\
\hline GLC-60 & & $\begin{array}{l}c_{20}: 0 \\
c_{20}: 1 \\
c_{20}: 2 \\
c_{20}: 3\end{array}$ & $\begin{array}{r}6.59 \\
7.48 \\
9.02 \\
11.28\end{array}$ & $\begin{array}{l}\text { Me. Arachidate } \\
\text { Me. Eicosemoate } \\
\text { Me. Eicosatrienoate } \\
\text { Me. Eicosatrienoate }\end{array}$ \\
\hline GLC-90 & & $\begin{array}{l}C_{13}: 0 \\
C_{15}: 0 \\
C_{17}: 0 \\
C_{19}: 0 \\
C_{21}: 0\end{array}$ & $\begin{array}{l}1.34 \\
2.00 \\
3.14 \\
5.07 \\
8.38\end{array}$ & $\begin{array}{l}\text { Me. Tridecanoate } \\
\text { Me. Tentadecanoate } \\
\text { Me. Heptadecanoate } \\
\text { Me. Nonadecanoate } \\
\text { Me. Hemeicosanoate }\end{array}$ \\
\hline PUFA-1 & & $\begin{array}{l}c_{14}: 0 \\
c_{16}: 0 \\
c_{16}: 1 \\
c_{18}: 0 \\
c_{18}: 1 \\
c_{18}: 2 \\
c_{20}: 1 \\
c_{22}: 1 \\
c_{20}: 5 \\
c_{24}: 1 \\
c_{22}: 5 \\
c_{22}: 6\end{array}$ & $\begin{array}{r}1.61 \\
2.48 \\
2.90 \\
3.95 \\
4.62 \\
5.52 \\
7.45 \\
12.09 \\
14.38 \\
18.90 \\
23.93 \\
26.15\end{array}$ & $\begin{array}{l}\text { Me. Myristate } \\
\text { Me. Palmitate } \\
\text { Me. Palmitoleate } \\
\text { Me. Stearate } \\
\text { Me. Oleate } \\
\text { Me. Linoleate } \\
\text { Me. Eicosenoate } \\
\text { Me. Erucate } \\
\text { Me. Eicosapentanoate } \\
\text { Me. Nervonate } \\
\text { Me. Docosahexaenoate }\end{array}$ \\
\hline
\end{tabular}


Fig. 52 Gas chromatographic profile of the fatty acid methyl esters obtained from surfactant \#1 after alkaline hydrolysis (saponification) by $\mathrm{KOH}$ in methanol, extraction with petroleum ether and derivatization by $\mathrm{BF}_{3}$ in methanol. 


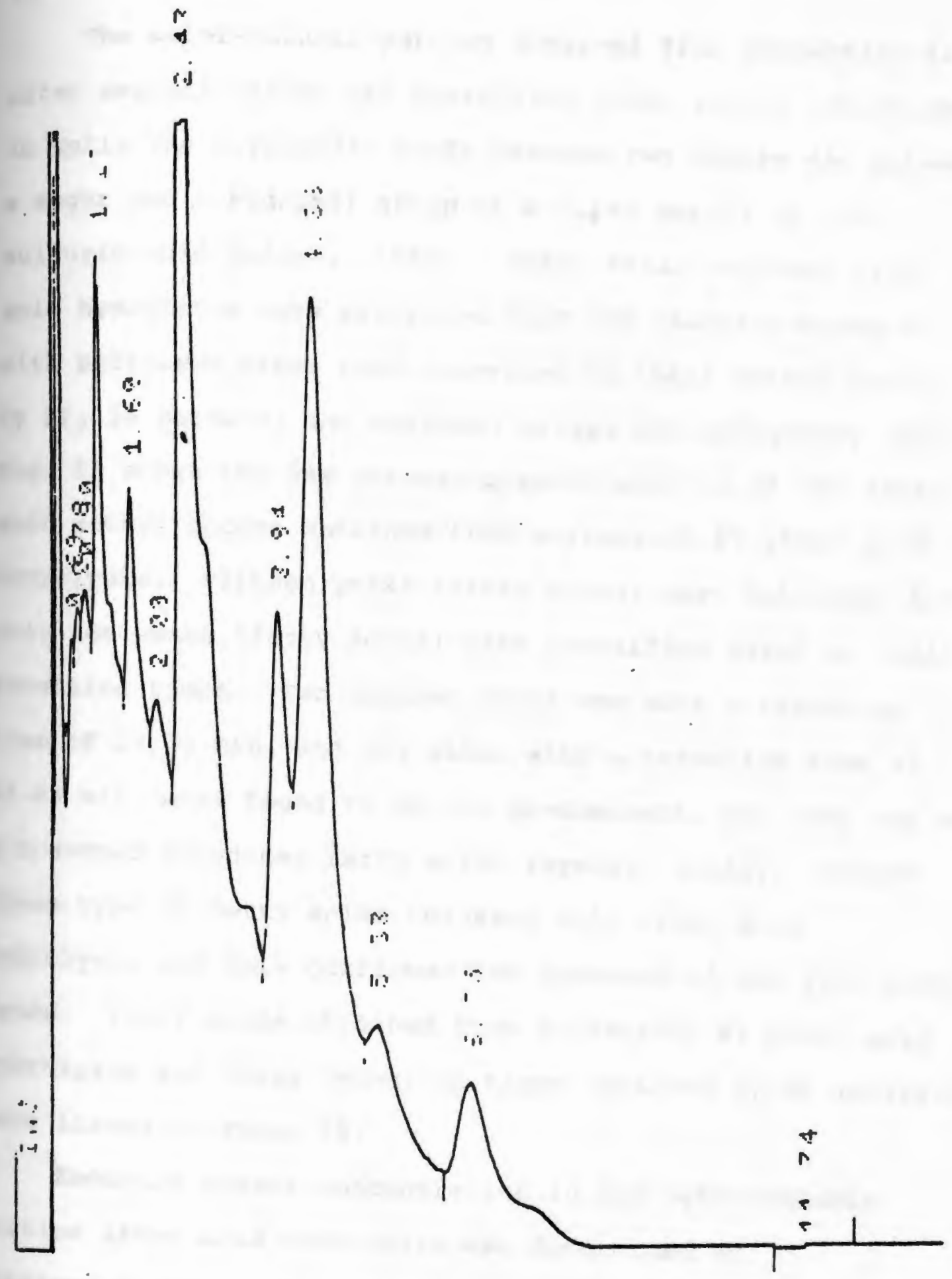


their correspondence retention times are summarized in Table 18.

The water-soluble portion obtained from surfactant \#1 after saponification was hydrolyzed under acidic conditions to split the glycosidic bonds between two sugars and between a sugar and a hydroxyl group of a lipid moiety by $72 \%$ sulfuric acid (Adam's, 1965). Fatty acids released after acid hydrolysis were extracted from the reaction mixture with petroleum ether then converted to their methyl esters by $\mathrm{BF}_{3}$ in meţhanol and analyzed by gas chromatography (GC). Fig. 53 shows the gas chromatographic profile of the fatty acid methyl esters obtained from surfactant \#1 after acid hydrolysis. Fifteen peaks (fatty acids) were detected, but only ten peaks (fatty acids) were identified based on their retention times. Two unknown peaks one with a retention time of $23.55 \mathrm{~min}$. and the other with a retention time of $63.63 \mathrm{~min}$. were found to be the predominant, and they may be $\alpha$-branched $\beta$-hydroxy fatty acids (mycolic acids), because these type of fatty acids released only after acid hydrolysis and this confirmed the presence of the glycosidic bands. Fatty acids obtained from surfactant \#1 after acid hydrolysis and their retention times obtained by GC analysis were listed in Table 19.

Reducing sugars concentration in the water-soluble portion after acid hydrolysis was determined by anthrone-sulfuric acid method (Dreywood, 1946) based on glucose as standard. The glucose standard curve shown by 
Table 18 Fatty acids obtained from surfactant \#1 after alkaline hydrolysis and their correspondence retention times as determined by GC.

$\begin{array}{lll}\text { RT } & \begin{array}{c}\text { \# of Carbons } \\ \text { of Double Bonds }\end{array} & \text { Fatty Acids } \\ 0.69 & C_{8}: 0 & \text { Me. Caprylate } \\ 0.78 & C_{10}: 0 & \text { Me. Caprate } \\ 0.90 & C_{11}: 0 & \text { Me. Undecanoate } \\ 1.10 & C_{12}: 0 & \text { Me. Laurate } \\ 1.60 & C_{14}: 0 & \text { Me. Myristate } \\ 2.00 & C_{15}: 0 & \text { Me. Pantadecanoate } \\ 2.43 & C_{16}: 0 & \text { Me. Palmitate } \\ 3.84 & C_{18}: 0 & \text { Me. Stearate } \\ 4.38 & C_{18}: 1 & \text { Me. Oleate } \\ 5.33 & C_{18}: 2 & \text { Me. Linoleate } \\ 6.75 & C_{20}: 0 & \text { Me. Arachidate }\end{array}$


Fig. 53 Gas chromatogram of the fatty acid methyl esters obtained from surfactant \#1 after acid hydrolysis and derivatization by $\mathrm{BF}_{3}$ in methanol. 


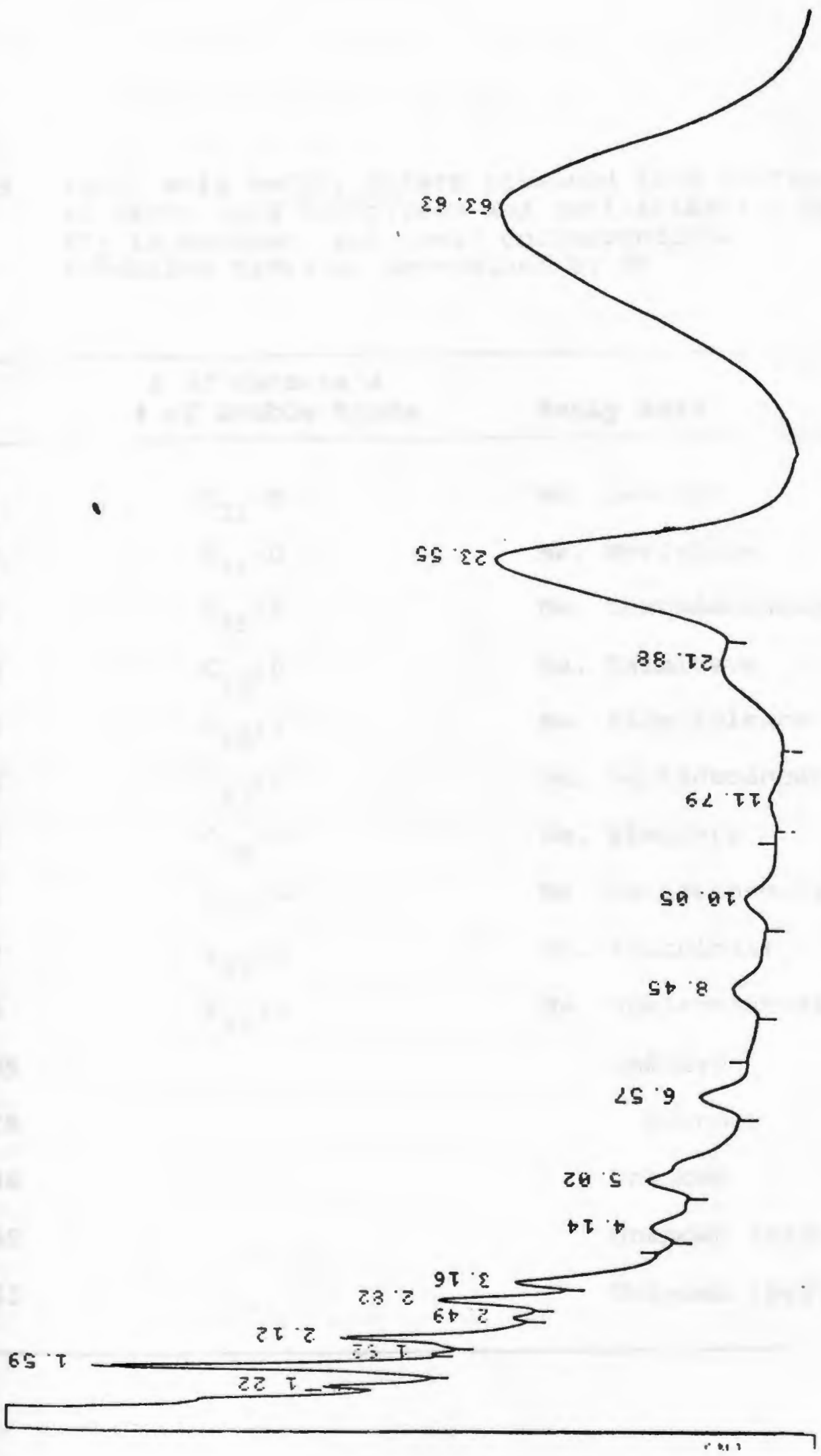


Table 19 Fatty acis methyl esters obtained from surfactant \#1 after acid hydrolysis and derivatization by $\mathrm{BF}_{3}$ in methanol and their correspondence retention times as determined by GC.

$\begin{array}{lll}\text { * of Carbons } & \begin{array}{l}\text { *f Double Bonds } \\ \text { of }\end{array} & \text { Fatty Acid } \\ 1.59 & c_{12}: 0 & \text { Me. Laurate } \\ 2.12 & c_{14}: 0 & \text { Me. Myristate } \\ 2.49 & c_{15}: 0 & \text { Me. Tentadecanoate } \\ 2.82 & c_{16}: 0 & \text { Me. Palmitate } \\ 3.16 & c_{16}: 1 & \text { Me. Palmitoleate } \\ 4.14 & c_{17}: 0 & \text { Me. Heptadecanoate } \\ 5.02 & c_{18}: 0 & \text { Me. Stearate } \\ 6.57 & c_{19}: 0 & \text { Me. Nonadecanoate } \\ 8.45 & c_{20}: 0 & \text { Me. Arachidate } \\ 10.05 & c_{21}: 0 & \text { Me. Hemiecosanoate } \\ 11.79 & - & \text { Unknown } \\ 21.88 & - & \text { Unknown } \\ 23.55 & - & \text { Unknown } \\ 63.63 & - & \text { Unknown (major) }\end{array}$


Fig. 54 was utilized for determination of reducing sugars concentration in $0.2 \mathrm{ml}$ of water soluble portion after alkaline and acid hydrolysis, and the reducing sugars concentration was $140 \mu \mathrm{g} / \mathrm{ml}$.

Sugars and sugar derivatives (mono-,di-, or trisaccharides), in the water-soluble portion from surfactant \#1 after acid hydrolysis were determined by comparison with authentic sample using high-pressure liquid chromatography. Twenty sugars and sugar derivatives were analyzed separately by HPLC to determine their relative retention times. These sugars and sugar derivatives and their correspondence retention times are summarized in Table 20. Fig. 55 shows the chromatogram obtained from analyzing the water-soluble portion, of surfactant \#1 after acid hydrolysis, by HPLC. Eight peaks were detected, but only three peaks were identified based on their retention times as raffinose, Galacturonic acid and the third peak with a retention time of $14.84 \mathrm{~min}$. may be identified as arabinose, $\mathbb{N - a c e t y l ~ g l u c o s e}$ amine, or fucose. Sugars and sugar derivatives obtained from surfactant \#1 after acids hydrolysis and their corresponding retention times, with other unidentified peaks were summarized in Table 21. Alkaline hydrolysis (saponification) of surfactant \#1 led to water-soluble sugars and water-insoluble lipid moities, this fact confirms the presence of ester bonds. The presence of ester bonds was also confirmed by absorption of UV-light at $209 \mathrm{~nm}$. Acid hydrolysis of surfactant \#1 also led to 

Gig. 54 Glucose standard curve, which was prepared by
anthrone-sulfuric acid method and utilized for determination of reducing sugar concentration of surfactant \#1 water soluble portion after acid hydrolysis.

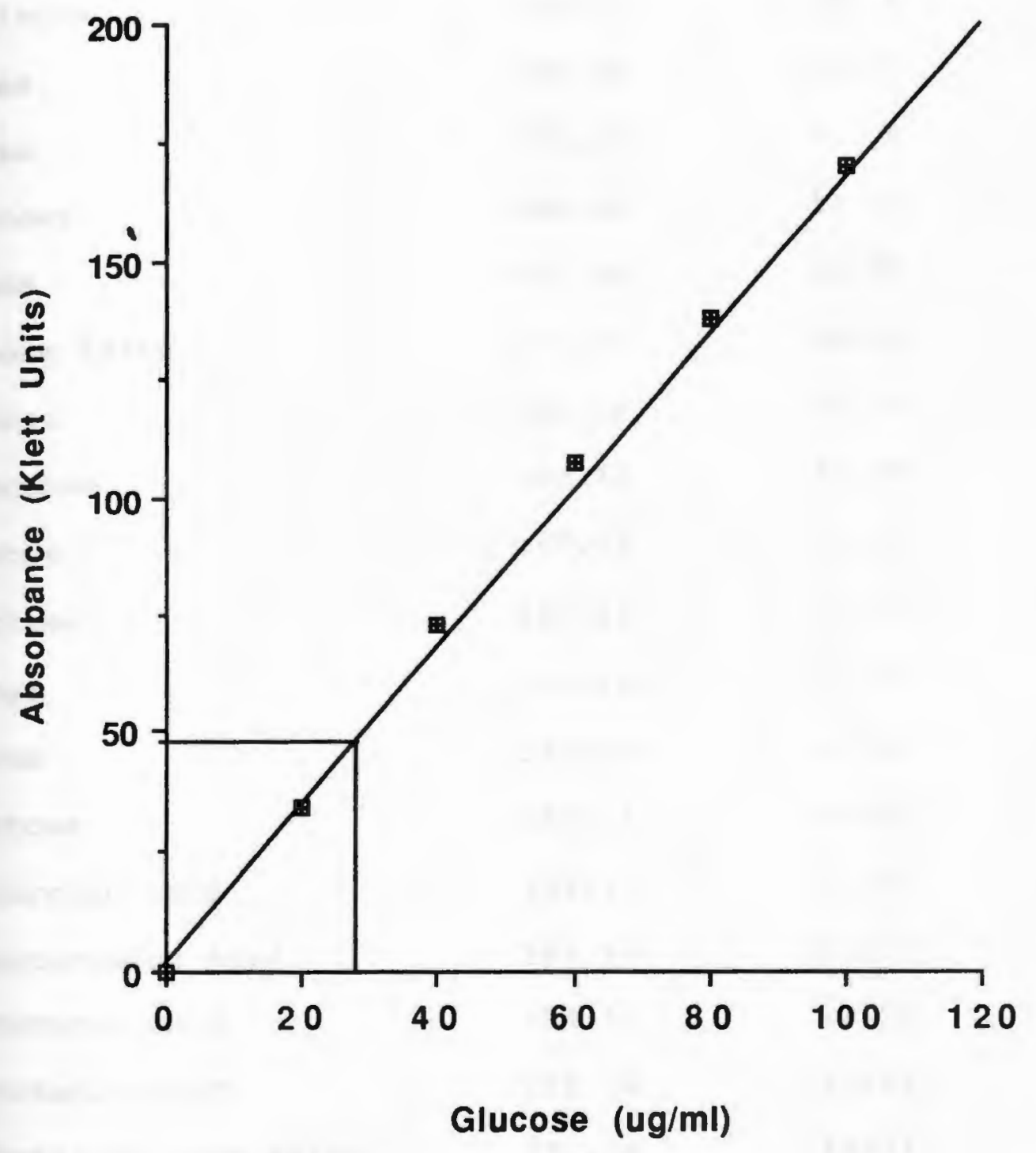


Table 20 Sugars and sugar derivatives as standards and their correspondence retention times as determined by HPLC, which was used for determination the relative retention times.

\section{Bugars}

encar Derivatives

M.W

RT

Arabinose

150.13

14.79

Ribose

150.13

15.73

Xylose

150.13

14.08

Rhamnose

164.16

13.58

Fucose

164.16

15.03

Glucose Amine

179.17

00.00

Glucose

280.16

12.51

Galactose

180.16

13.50

Mannose

180.16

13.48

Fructose

180.16

13.71

Gulose

180.16

13.30

Talose

180.16

15.48

Tagatose

180.16

12.94

Glucuronic Acid

194.14

11.24

Galacturonioc Acid

194.14

12.33

Mannuronic Acid

194.14

16.59

Galactonic Acid

196.16

12.41

N-Acetyl Glucose Amine

221.16

14.71

Melibiose

342.30

10.56

Raffinose

504.46

9.72 

Fig. 55 High pressure liquid chromatographic profile of portion obtained from surfactant $\# 1$ after acid
hydrolysis.

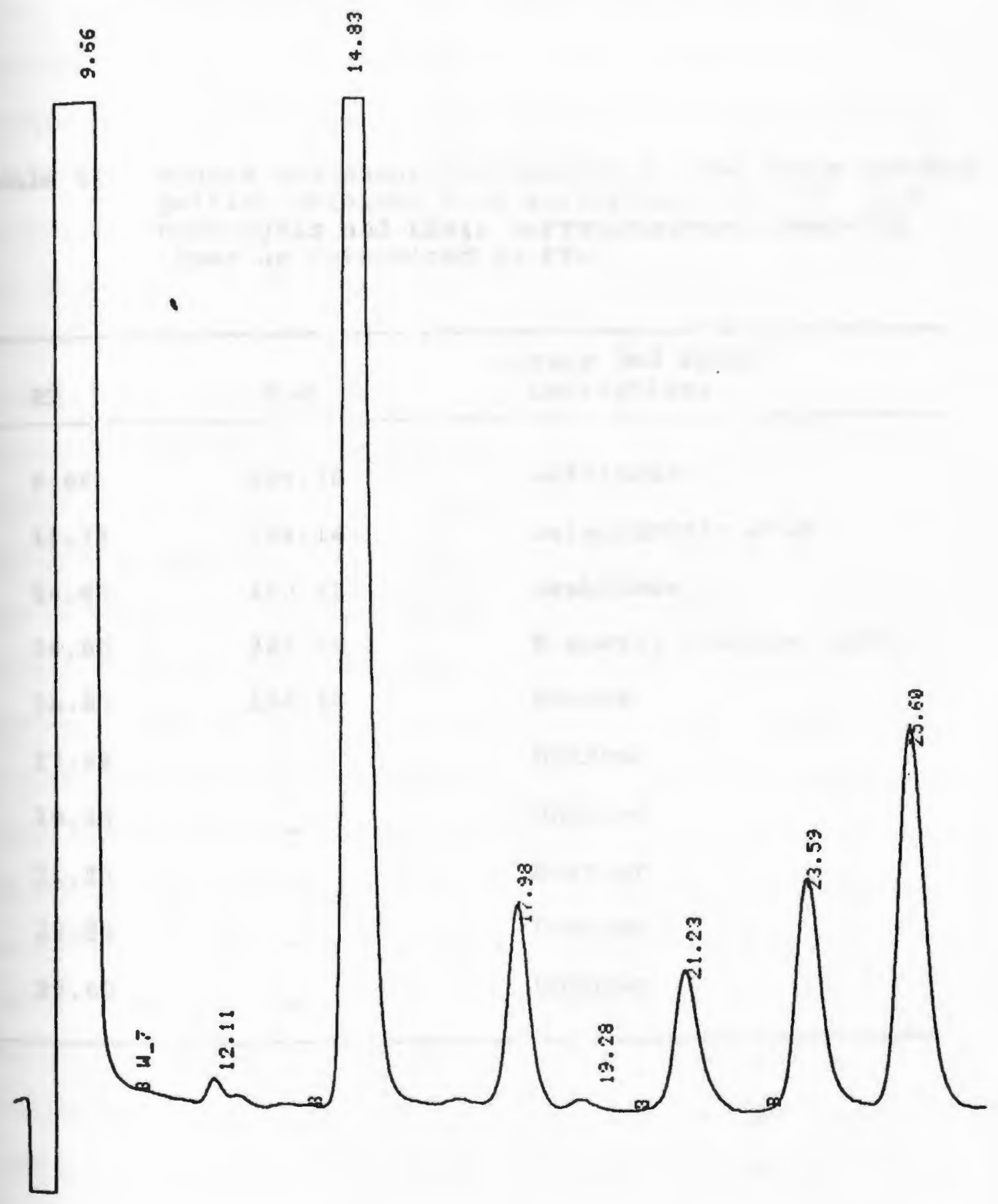


Table 21 Sugars and sugar derivatives in the water soluble portion obtained from surfactnat \#1 after acid hydrolysis and their correspondence retention times as determined by HPLC.

\begin{tabular}{lll} 
RT & M.W. & $\begin{array}{c}\text { sugars and sugar } \\
\text { Derivatives }\end{array}$ \\
\hline 9.66 & 504.46 & Raffinose \\
12.11 & 194.14 & Galacturonic Acid \\
14.83 & 150.13 & Arabinose \\
14.83 & 221.16 & N-Acetyl Glucose Amine \\
14.83 & 164.16 & Fucose \\
17.98 & - & Unknown \\
19.28 & - & Unknown \\
21.23 & - & Unknown \\
23.59 & - & Unknown \\
25.60 & - & Unknown \\
\hline
\end{tabular}


water-soluble sugars and water-insoluble lipids and this confirms the presence of glycosidic bonds between sugars and a hydroxyl groups of a lipid moities. All of these results confirms that surfactant \#1 is a glycolipid.

Surfactant \#1 which was eluted by methanol as the mobile phase from the silica gel column as stationary phase stayed at the base line of the silica gel G type TLC plate after development by hexane, chloroform, ethyl acetate and methanol $(30,40,20,10)$, therefore, the purity of surfactant \#1 is questionable. In order to confirm the purity, surfactant \#1 should move up from the base line of the TLC plate. The only way to move the surfactant \#1 up from the base line was through changing the polarity of the mobile phase (i.e. increasing the polarity) used for developing the TLC plate, and this was preformed by changing the ratios of the organic solvents by increasing the polar solvents such as ethyl acetate and methanol and decreasing the non-polar solvents such as hexane and chloroform. As a result, one mobile phase hexane, chloroform, ethyl acetate and methanol $(20,30,20,30)$ was found to give the best results. Developing the TLC plate with this mobile phase moved surfactant \#1 from the base line and separated it into three bands which were detected by vanillin reagent. These results indicates that surfactant \#1 was not pure and therefore further purification by column chromatography was required before the characterization of the biosurfactant. 


\section{Purification of the biosurfactant by column}

\section{chromatography :}

For further purification of surfactant \#1 (which was eluted by methanol as mobile phase from the silica gel column), $0.5 \mathrm{gm}$ of the freeze dried powder (surfactant \#1) was dissolved in $5 \mathrm{ml}$ methanol, then introduced for purifaction onto a $\mathrm{C}_{18}$ silica column (reverse phase column chromatography) as stationary phase. Two mobile phases, pure methanol and chloroform and methanol $(2: 1)$ were used to elute the surfactant \#1 components from the $\mathrm{C}_{18}$ silica column. As a result, surfactant \#1 components were separated into two bands, which were detected during elution from the column by UV-light. One band was eluted first with methanol as a mobile phase and the other band was eluted later with chloroform and methanol. (2:1).

The purity of the two bands which were eluted from the $\mathrm{C}_{18}$ silica column was tested by silica gel G type TLC plates, which were developed by hexane, chloroform, ethyl acetate and methanol $(20: 30: 20: 30)$ as the mobile phase and detected by vanillin reagent, anthrone sulfuric acid, iodine vapours and UV-light. The band which eluted with methanol moved about $2 \mathrm{~cm}$ up from the base line on TLC plate, and the band which eluted from $c_{18}$ silica column by chloroform and methanol (2:1), separated into two bands on the TLC plate.

The surface activity was tested for both bands, the first band, which eluted by methanol from the $c_{18}$ silica column, was found to decrease the surface tension of the 
dist. $\mathrm{H}_{2} \mathrm{O}$ from 73:0 to 27.8 dynes/cm and the interfacial tension from 18.0 to 2.3 dynes/cm. The second band, which eluted by chloroform and methanol (2:1) later from $\mathrm{C}_{18}$ silica column, was found to decrease the surface tensnion of the dist. $\mathrm{H}_{2} \mathrm{O}$ only from 73.0 to $62.4 \mathrm{dynes} / \mathrm{cm}$ and the interfacial tension from 18.0 to 14.3 dynes/cm. These results indicated that the surface activity was associated with the band eluted with methanol as a mobile phase from $\mathrm{C}_{18}$ silica column.

Since, the purity of the band eluted by methanol was confirmed by TLC test, and also the surface activity was confirmed to be associated with this band, methanol was evaporated by vacuum evaporator at $40^{\circ} \mathrm{C}$ and the residue was dissolved in water and freeze dried. The freeze dried powder was designated AL-Hazmi's surfactant.

The correlation between AL-Hazmi's surfactant concentration and the surface activity:

AL-Hazmi's surfactant in the range of $0.2-2.0 \mathrm{mg} / 100 \mathrm{ml}$ with $0.2 \mathrm{mg} / 100 \mathrm{ml}$ increments were used to determine the relationship between $\mathrm{AL}$-Hazmi's surfactant concentration and the reduction of surface and interfacial tensions. Surface and interfacial tensions decreased with the increase of AL-Hazmi's surfactant concentration to $1.8 \mathrm{mg} / 100 \mathrm{ml}$, then any further increase in the concentration beyond this point resulted in no substantial reduction in surface and interfacial tensions as shown by Fig. 56 a, b. This point is the critical micelle concentration (CMC). The surface 
Fig. 56 a,b The correlation between AL-Hazmi surfactant concentration and surface activity as determined by the reduction of surface and interfacial tensions by Fisher autotensiomat. 

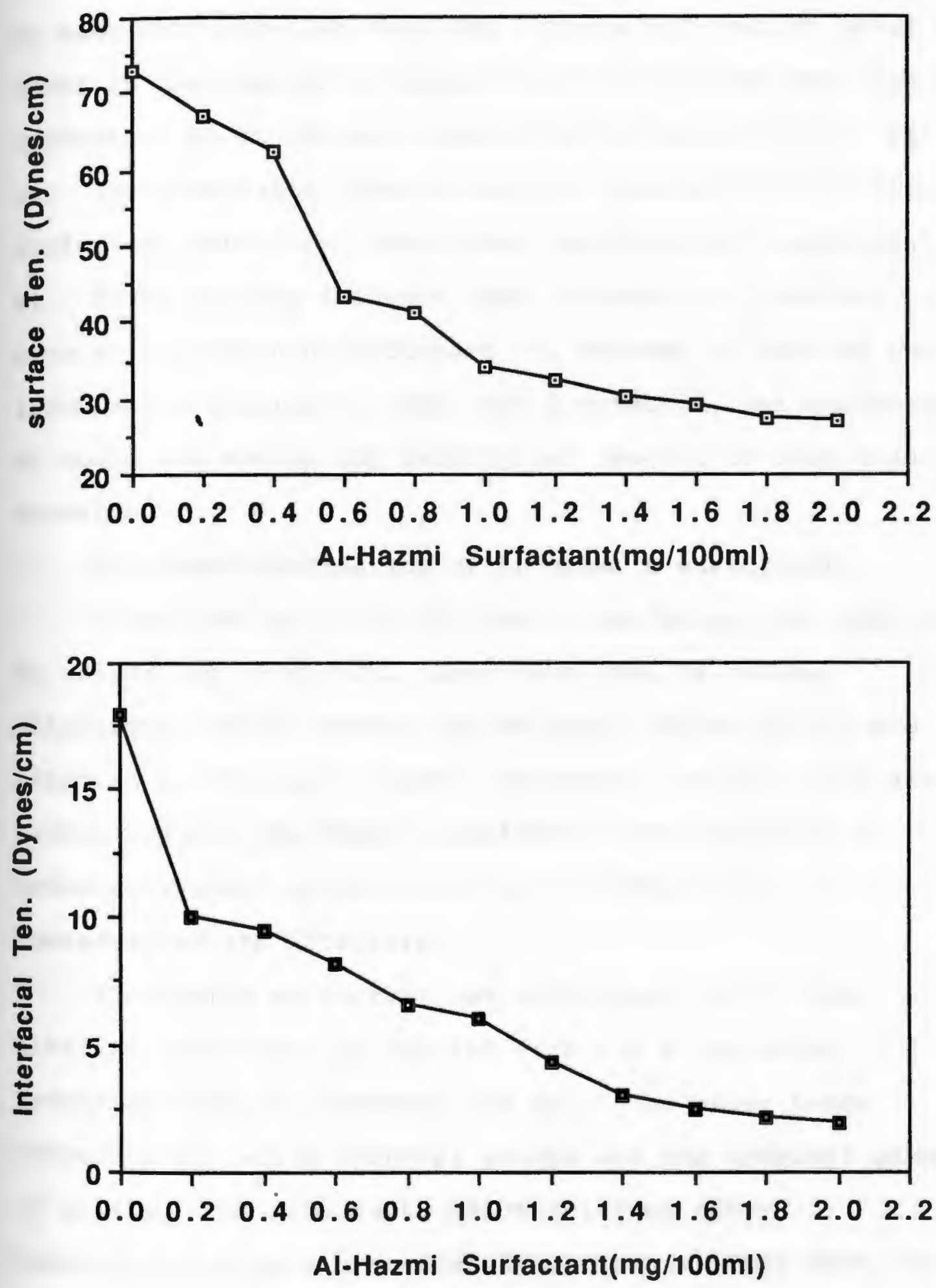
tension value obtained from AL-Hazmi surfactant at 1.6 $\mathrm{ml} / 100 \mathrm{ml}$ was higher than that obtained from surfactant \#1 by same concentration, but the interfacial tension value was lower in the case of AL-Hazmi's surfactant than the case of surfactant \#1 at the same concentration which was $1.6 \mathrm{mg} / 100$ ml. The interfacial tension values obtained from AL-Hazmi's surfactant were lower than those obtained from surfactant \#1. These results indicate that Al-Hazmi's surfactant is more effective than surfactant \#1, because it reduces the interfacial, tension to less than 2 dynes/cm, but surfactant \#1 could not reduce the interfacial tension to less than 3.0 dynes/cm.

Structure elucidation of AL-Hazmi's surfactant:

After the purity of AL-Hazmi's surfactant was confirmed by silica gel G type TLC plate developed by hexane, chloroform, ethyl acetate and methanol $(20: 30: 20: 30)$ and detected by vanillin reagent, anthrone, surfuric acid and iodine vapours, AL-Hazmi's surfactant was subjected to hydrolysis under alkaline and acidic conditions, to characterize its structure.

AL-Hazmi's surfactant was hydrolyzed first under alkaline condition, by heating with $0.5 \mathrm{~N}$ potassium hydroxide $(\mathrm{KOH})$ in methanol, to split the ester bonds between fatty acids carboxyl groups and the hydroxyl groups of sugars. The free fatty acids released after saponification were extracted by petroleum ether from the acidified reaction mixture, then converted to their methyl 
esters by $\mathrm{BF}_{3}$ in methanol. The resultant fatty acid methyl esters were analyzed and the distribution of the fatty acids were determined by a Varian gas chromatograph equipped with a flame ionization detector and $10 \% \mathrm{sp}-2330$ (Cyano silicon) column. Fatty acids obtained from AL-Hazmi surfactant after saponification were identified based on their retention times (by comparing their retention times with those obtained from the standards, which were summerized in Table 16 and 17). Fig. 57 shows the gas chromatographic profile of the fatty acids obtained from AL-Hazmi's surfactant after saponification. Seven fatty acids were detected and identified as $\mathrm{C}_{12: 0}, \mathrm{C}_{14: 0}, \mathrm{C}_{16: 0}, \mathrm{C}_{16: 1}, \mathrm{C}_{17: 0}, \mathrm{C}_{18: 0}$ and $\mathrm{c}_{18: 1}$, but only three fatty acids $\mathrm{c}_{16: 0,} \mathrm{C}_{16: 1}$ and $\mathrm{c}_{18: 1}$ were predominant as shown by Fig. 55. Fatty acids and their correspondence retention times were summarized in Table 22.

The water soluble portion, which remained after saponification and extraction of free fatty acids, was hydrolyzed under acidic condition to split the glycosidic bonds between two sugars or between a sugar and a hydroxyl group of a lipid moiety, by $72 \%$ sulfuric acid. The free fatty acids released after acid hydrolysis were extracted by petroleum ether then converted to their methyl esters by $\mathrm{BF}_{3}$ in methanol and analyzed by gas chromatograph. Fig. 58 shows the gas chromatogram of the fatty acid methyl esters obtained from AL-Hazmi's surfactant after acid hydrolysis. Thirteen peaks were detected, but only ten fatty acids were identified based on their retention times (through comparing 
Fig. 57 Gas chromatogram of the fatty acid methyl esters obtained from AL-Hazmi surfactant after alkaline hydrolysis bo $\mathrm{KOH}$ in methanol and derivatization by $\mathrm{BF}_{3}$ in methanol.

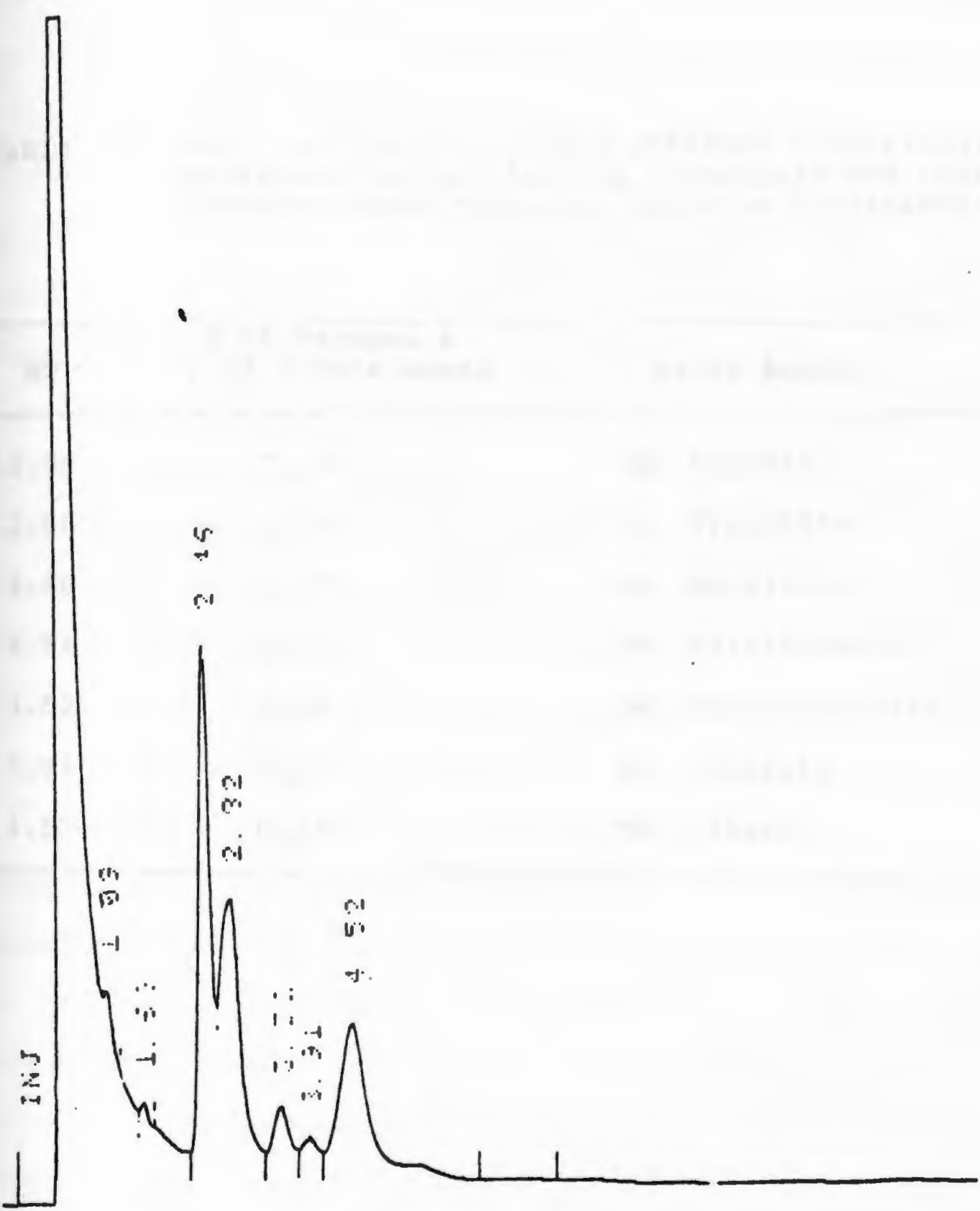


Table 22 Fatty acid methyl esters obtained from Al-Hazmi surfactant after alkaline hydrolysis and their correspondence retention times as determined by GC.

\begin{tabular}{lll}
\hline RT & $\begin{array}{c}\text { \# of Carbons } \\
\text { of Double Bonds }\end{array}$ & \multicolumn{1}{c}{ Fatty Acids } \\
\hline 1.09 & $C_{12}: 0$ & Me. Laurate \\
1.60 & $C_{14}: 0$ & Me. Myristate \\
2.46 & $C_{16}: 0$ & Me. Palmitate \\
2.82 & $C_{16}: 1$ & Me. Palmitoleate \\
3.52 & $C_{17}: 0$ & Me. Haptadecanoate \\
3.91 & $C_{18}: 0$ & Me. Stearate \\
4.52 & $C_{18}: 1$ & Me. Oleate \\
\hline
\end{tabular}


their retention times with those obtained from the standards summarized in Table 16 and 17). Two unknown peaks were detected at a retention times of 19.24 and $51.11 \mathrm{~min}$. were the predominant, they may be $\alpha$-branched $\beta$-hydroxy fatty acids (mycolic acids), because they were released only after acid hydrolysis and this means that they were linked to sugars by a glycosidic bonds through their hydroxyl groups, therefore these results confirmed the presence of a glycosidic bonds. Fatty acids obtained from AL-Hazmi's surfactant after acid hydrolysis and their correspondence retention times were summarized in Table 23.

Reducing sugars concentration, in the water soluble portion after acid hydrolysis were determined by anthrone-sulfuric acid method based on glucose as a standard. Glucose standard curve shown by Fig. 59 was utilized for determination of reducing sugar concentration in $0.2 \mathrm{ml}$ of the water soluble fraction, and the reducing sugar concentration was $85 \mu \mathrm{g} / \mathrm{ml}$.

Sugars and sugar derivatives contents of the water soluble portion after acid hydrolysis were analyzed by high pressure liquid chromatography (HPLC), and identified based on their retention times (through comparing their retention times with those of the standards in Table 20). Six peaks were detected by HPLC as shown by Fig. 60 , but only two peaks (sugars) were identified as raffinose, galacturonic acid and the third peak with a retention time of $14.84 \mathrm{~min}$. 
Fig. 58 Gas chromatographic profile of the fatty acid methyl esters obtained from Al-Hazmi surfactant after acid hydrolysis using $72 \% \mathrm{H}_{2} \mathrm{SO}_{4}$, extraction with petroleum ether and derivatization by $\mathrm{BF}_{3}$ in methanol. 


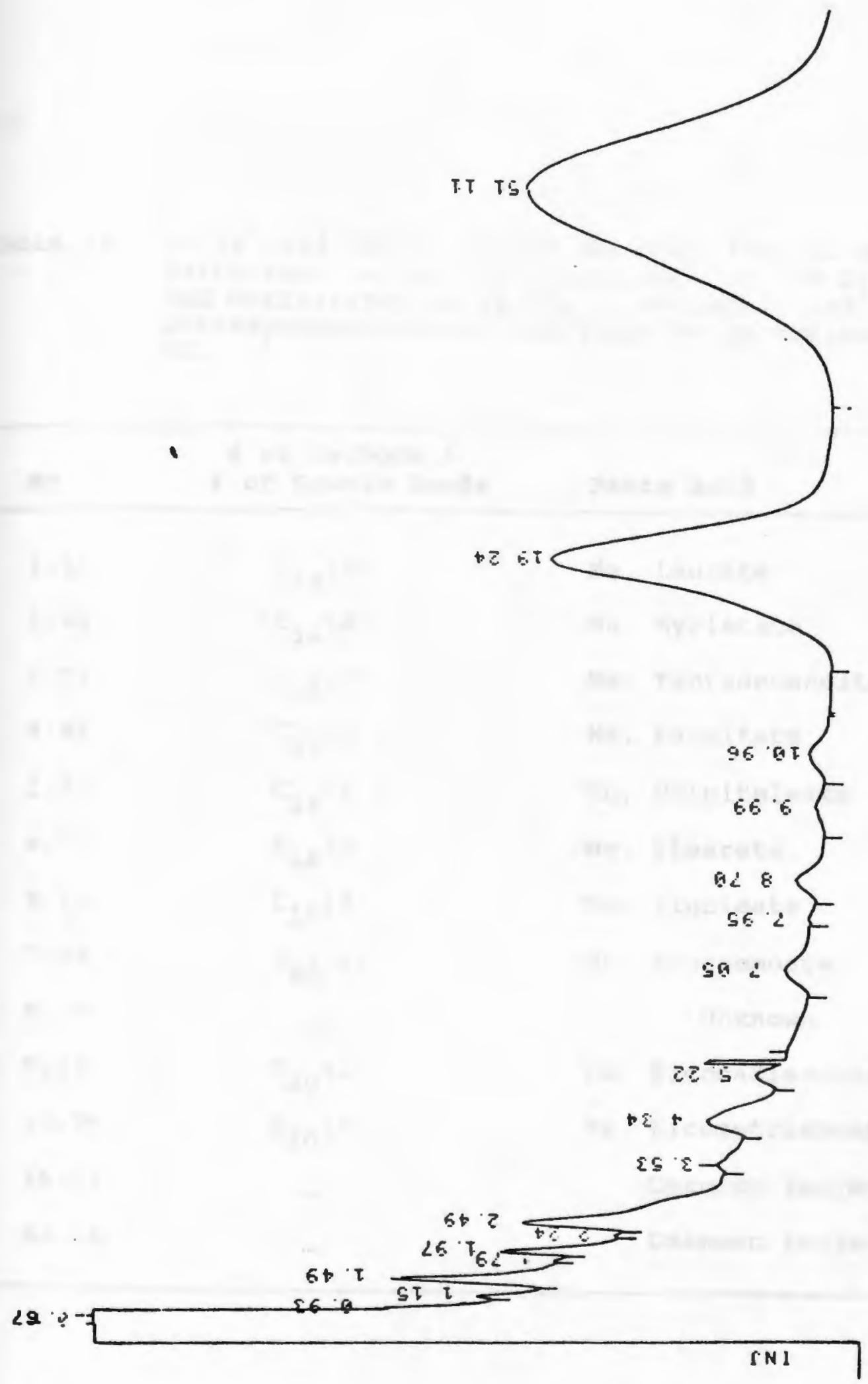


Table 23 Fatty acid methyl esters obtained from AL-Hazmi surfactant, after acid hydrolysis by $72 \% \mathrm{H}_{2} \mathrm{SO}_{4}$ and derivatization by $\mathrm{BF}_{3}$ in methanol, and their correspondence retention times as determined by GC.

\begin{tabular}{lll}
\hline RT & $\begin{array}{c}\text { \# of Carbons } \\
\text { of Double Bonds }\end{array}$ & Fatty Acid \\
\hline 1.15 & $c_{12}: 0$ & Me. Laurate \\
1.49 & $c_{14}: 0$ & Me. Myristate \\
1.97 & $c_{15}: 0$ & Me. Tentadecanoate \\
2.49 & $c_{16}: 0$ & Me. Palmitate \\
3.53 & $c_{16}: 1$ & Me. Palmitoleate \\
4.34 & $c_{18}: 0$ & Me. Stearate \\
5.22 & $c_{18}: 2$ & Me. Linoleate \\
7.05 & $c_{20}: 1$ & Me. Eicosemoate \\
8.70 & - & Unknown \\
9.99 & $c_{20}: 2$ & Me. Eicosadienoate \\
10.96 & $c_{20}: 3$ & Me. Eicosetrienoate \\
19.24 & - & Unknown (major) \\
51.11 & - & Unknown (major) \\
\hline
\end{tabular}


Fig. 59 Glucose standard cruve prepared by anthrone-sulfuric acid method and utilized for determination the reducing sugar concentration in $0.2 \mathrm{ml}$ of water soluble portion obtained from Al-Hazmi surfactant after acid hydrolysis.

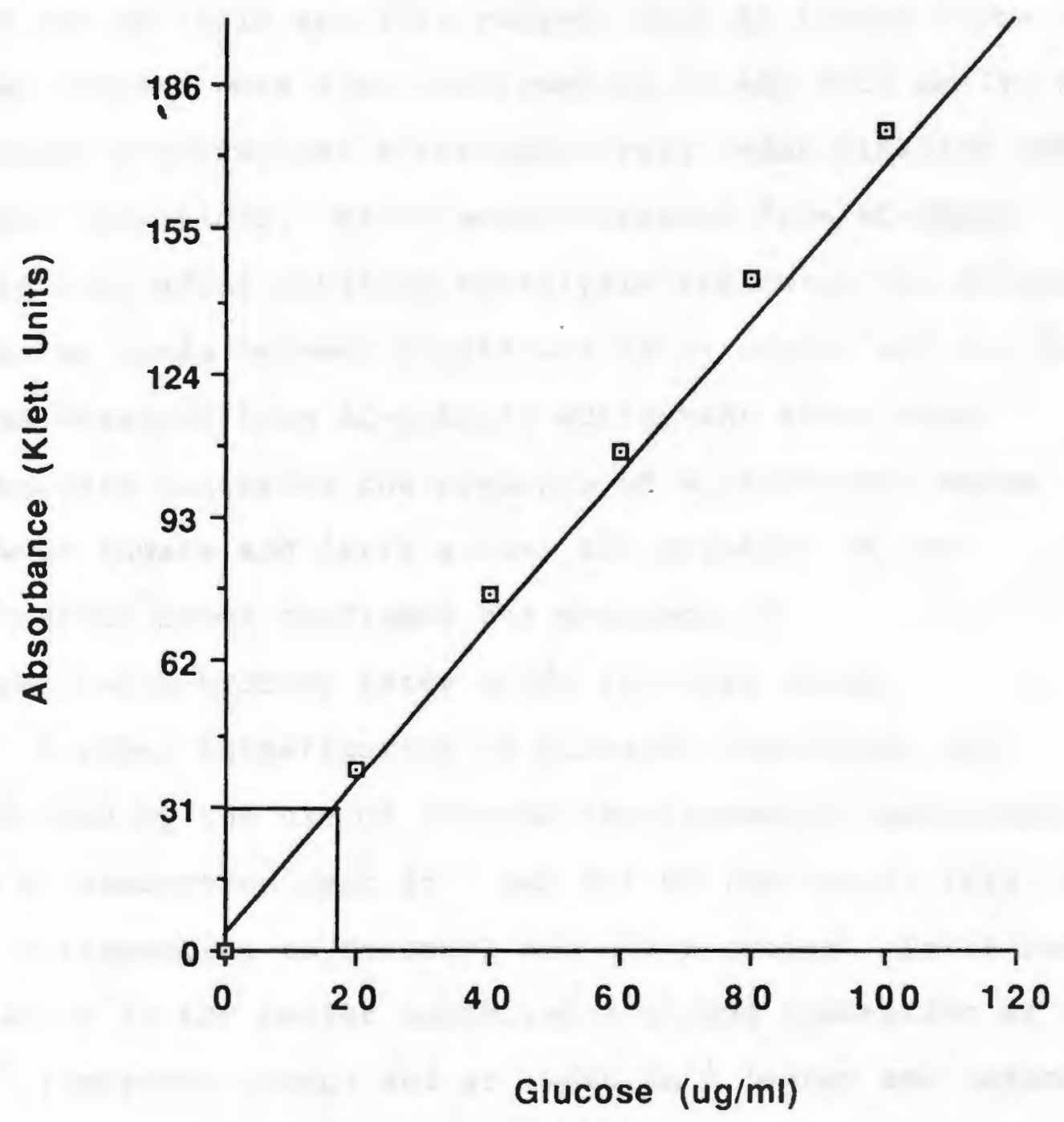


could be identified as arabinose, N-acetyl glucose amine or fucose. Sugars, sugar derivatives and unknowns obtained from AL-Hazmi's surfactant and their correspondence retention times are summarized in Table 24 .

The results obtained from TLC plate indicates that AL-Hazmi's surfactant was pure because it gave only one band on the TLC plate, and was a glycolipid because it was detected by sugar specific reagent such as anthrone-sulfuric acid and by lipid specific reagent such as iodine vapours. These results were also confirmed by GC and HPLC analysis of AL-Hazmi's surfactant after hydrolysis under alkaline and acidic conditions. Fatty acids obtained from AL-Hazmi surfactant after alkaline hydrolysis indicates the presence of ester bonds between sugars and fatty acids, and the fatty acids obtained from AL-Hazmi's surfactant after acid hydrolysis indicates the presence of a glycosidic bonds between sugars and fatty acids, the presence of the glycosidic bonds confirmed the presence of $\alpha$-branched- $\beta$-hydroxy fatty acids (mycolic acids).

Further investigation of AL-Hazmi surfactant was conducted by the use of various spectroscopic techniques. The UV absorption peak at $\max 206 \mathrm{~nm}$ (methanol) (Fig. 61) is corresponding to carboxyl and ester groups. Infra red analysis in $\mathrm{KBr}$ pellet exhibited a strong absorption at 3300 $\mathrm{Cm}^{-1}$ (hydroxyI group) and at $1660 \mathrm{~cm}^{-1}$ (ester and carboxyl groups) (Fig. 62). $1_{\mathrm{H}}$ and ${ }^{13^{3}} \mathrm{C}$-nuclear magnatic resonance 
Fig. 60 High pressure liquid chromatographic profile of the sugars and sugar derivatives in the water soluble portion, obtained from AL-Hazmi surfactant after acid hydrolysis. 


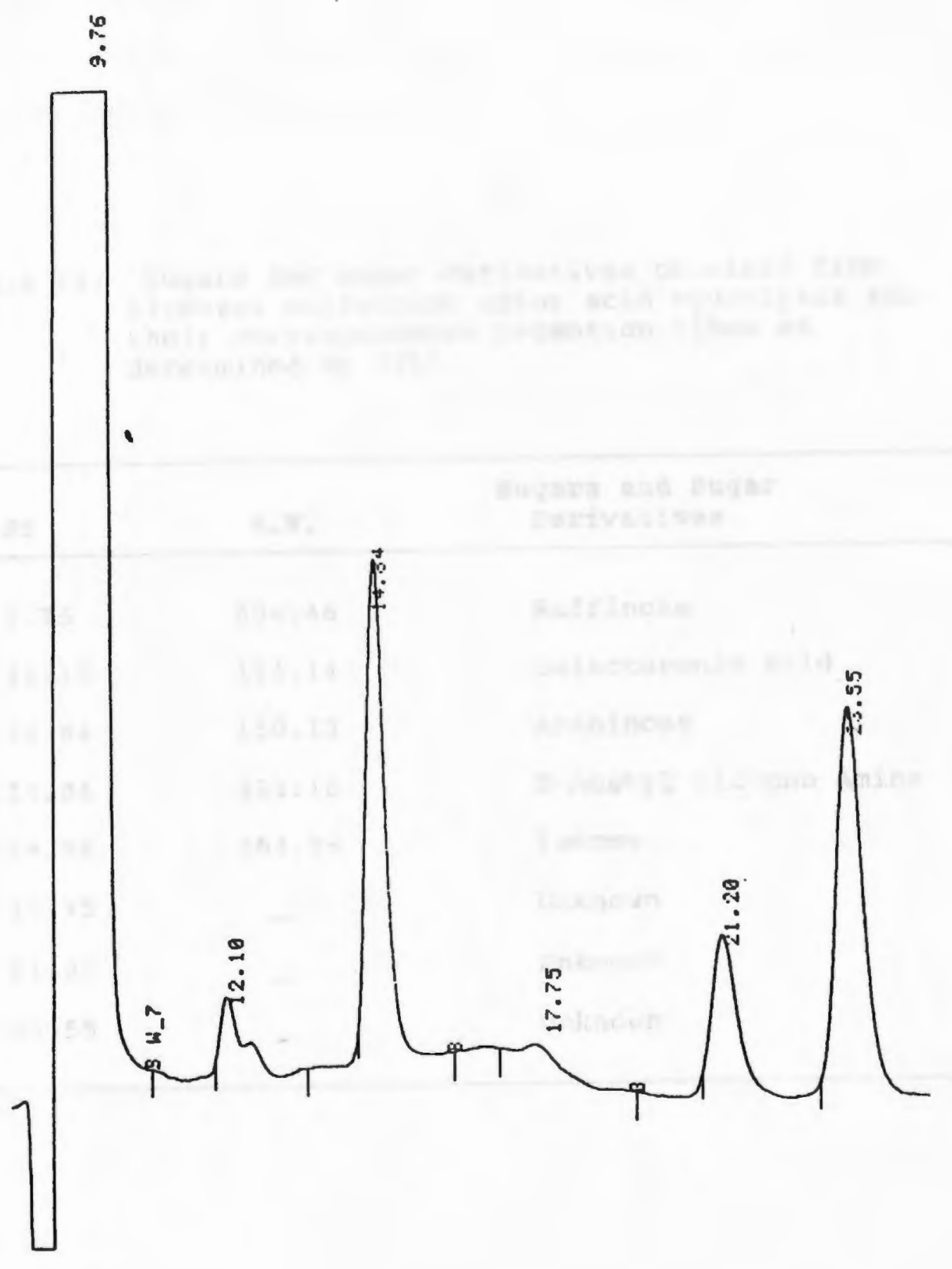

$-237-$ 
Table 24. Sugars and sugar derivatives obtained from Al-Hazmi surfactant after acid hydrolysis and their correspondence retention times as determined by HPLC.

\begin{tabular}{lll}
\hline RT & M.W. & $\begin{array}{c}\text { Bugars and sugar } \\
\text { Derivatives }\end{array}$ \\
\hline 9.76 & 504.46 & Raffinose \\
12.10 & 194.14 & Galacturonic Acid \\
14.84 & 150.13 & Arabinose \\
14.84 & 221.16 & N-Acetyl Glucose Amine \\
14.84 & 164.16 & Fucose \\
17.75 & - & Unknown \\
21.20 & - & Unknown \\
23.55 & - & Unknown \\
\hline
\end{tabular}


(NMR) spectrum (MeOH-d4) shows the existance of $\mathrm{CH}, \mathrm{CH}_{2}$ and $\mathrm{CH}_{3}$ type of proton signals as shown by Fig 63, along with obvious ketonic peaks in the ${ }^{13} \mathrm{C}$ chart shown in Fig. 64 . This is consistent with the normal splitting pattern of similar types of glycolipids. 
Fig. 61 UV-spectra of Al-Hazmi surfactant, which was scanned between $\max$ 900-190 nm using Lambda 4B UV/VIS spectrophotometer. 


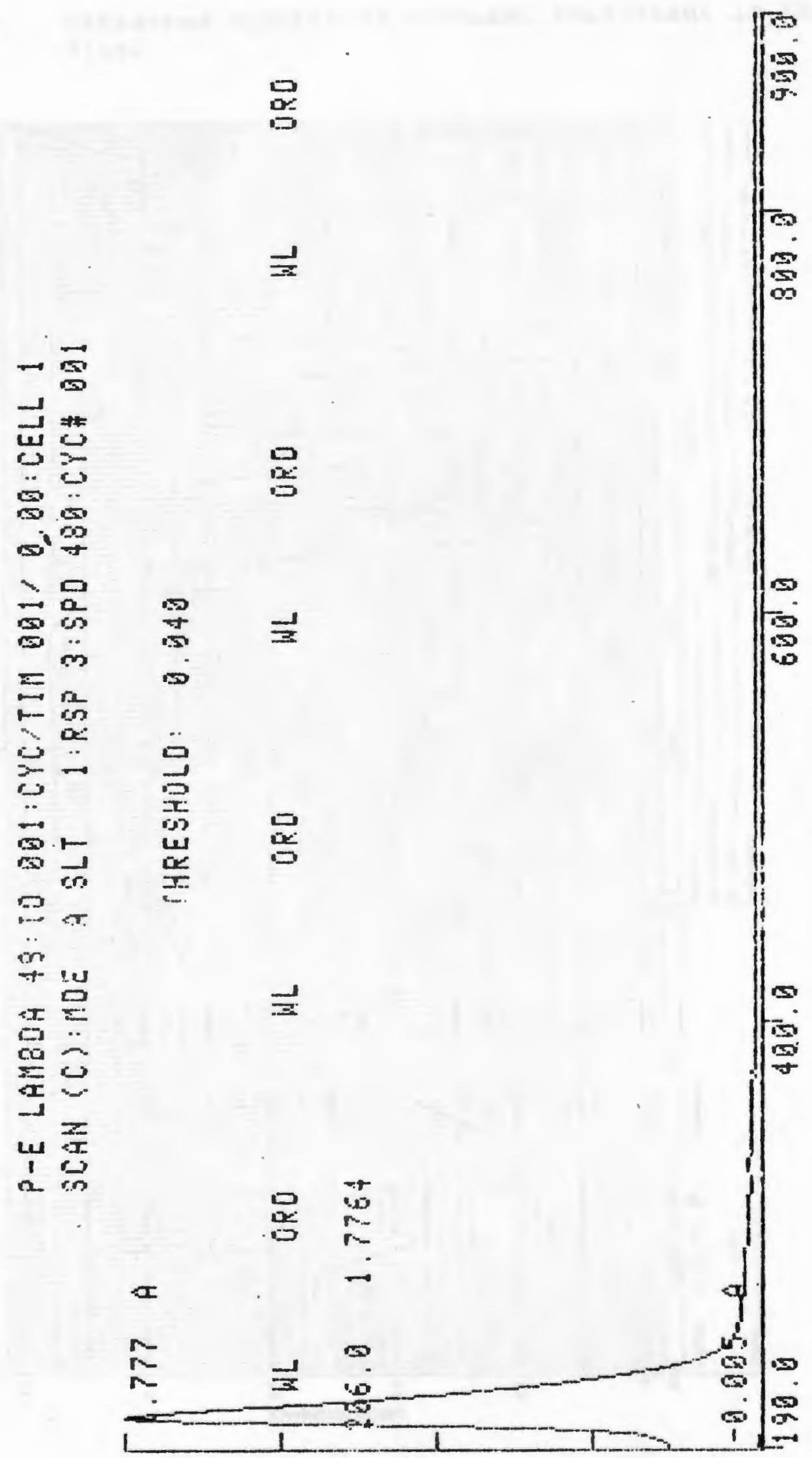




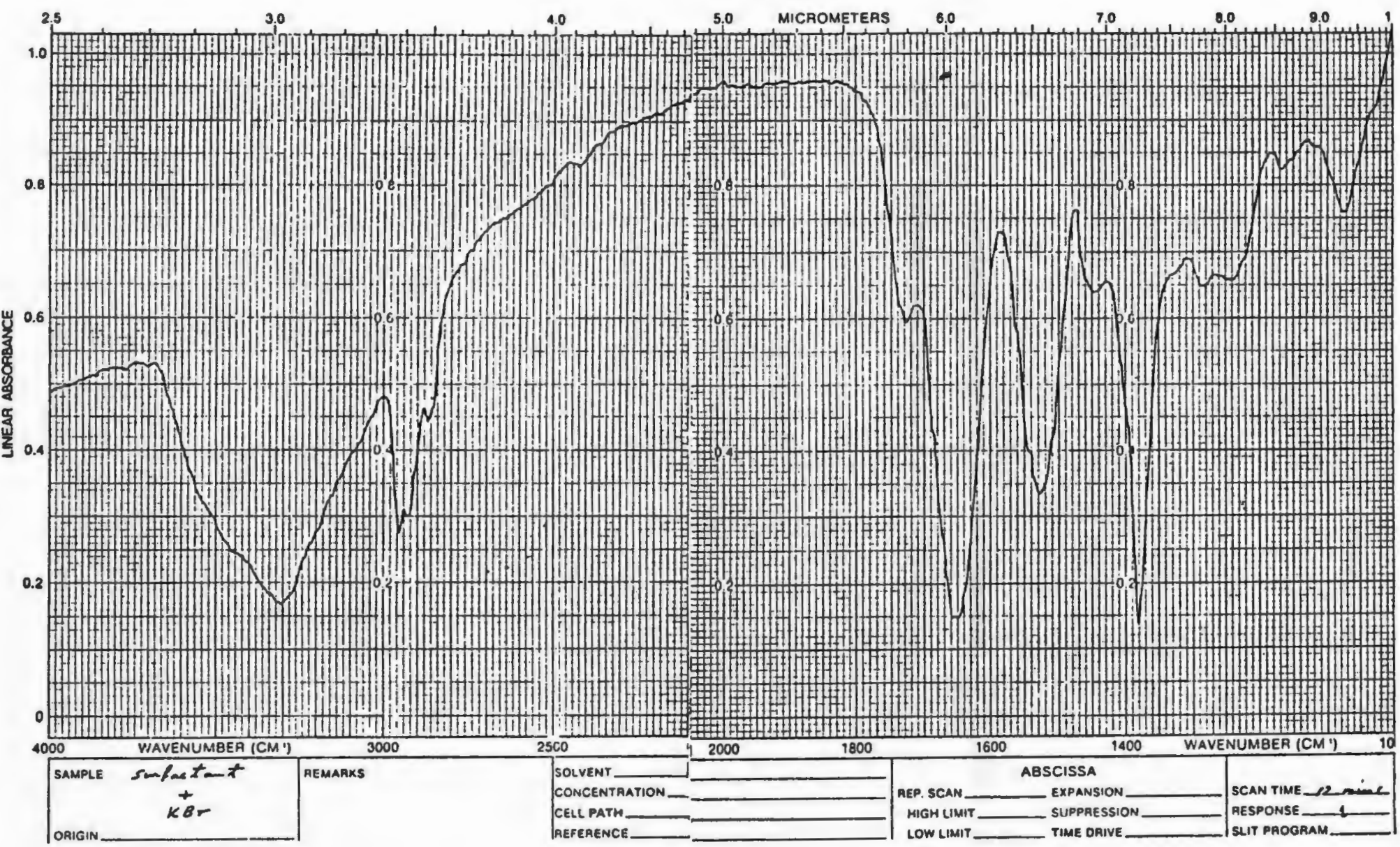


Fig. 63 Proton nuclear magnetic resonance (1 ${ }_{\mathrm{H}}$ NMR) spectra 1 of Al-Hazmi surfactant. 


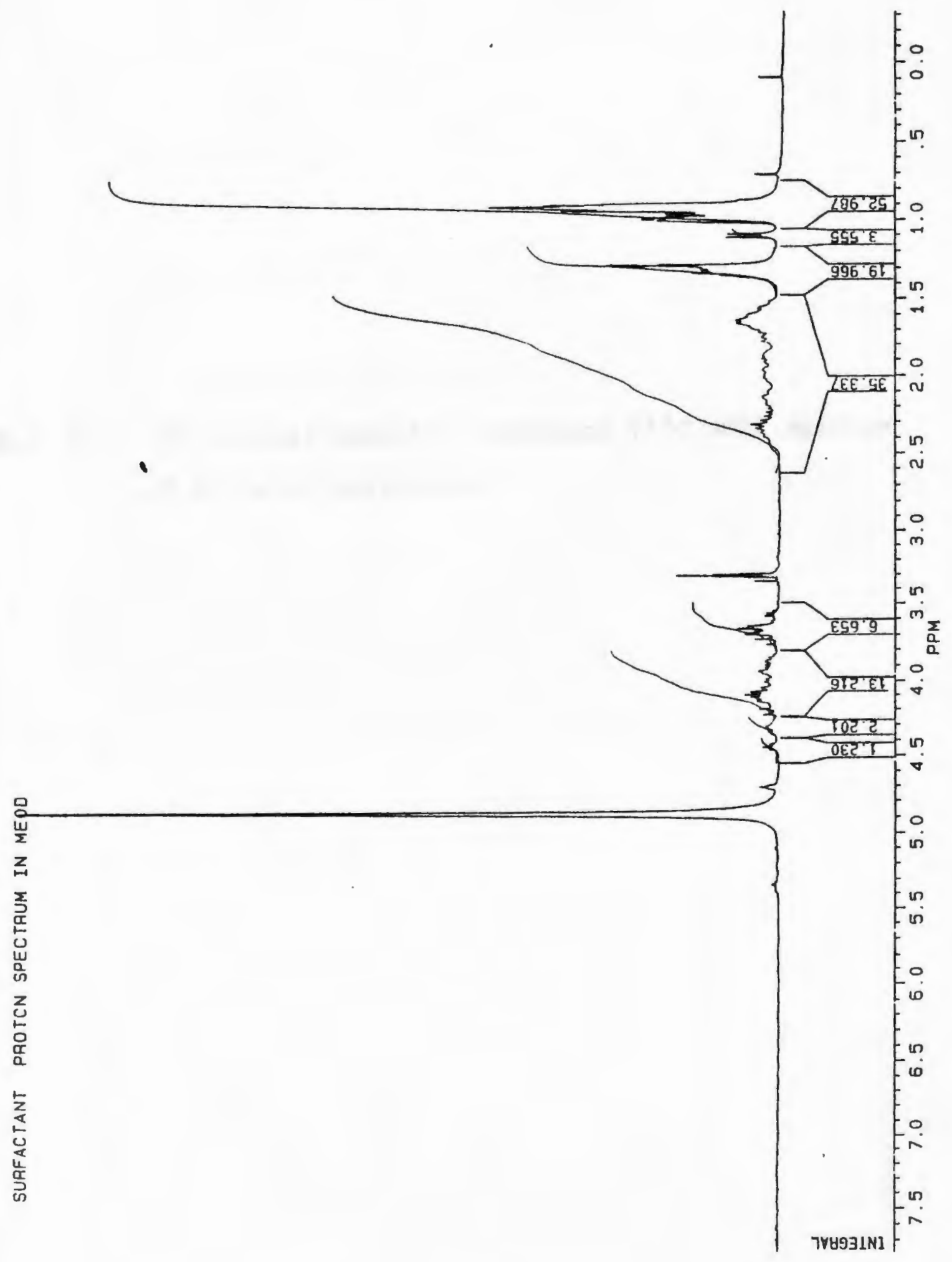


Fig. $64 \quad{ }^{13} \mathrm{C}$ Nuclear magnetic resonance $\left({ }^{13} \mathrm{C}\right.$ NMR $)$ spectra 1 of Al-Hazmi surfactant. 


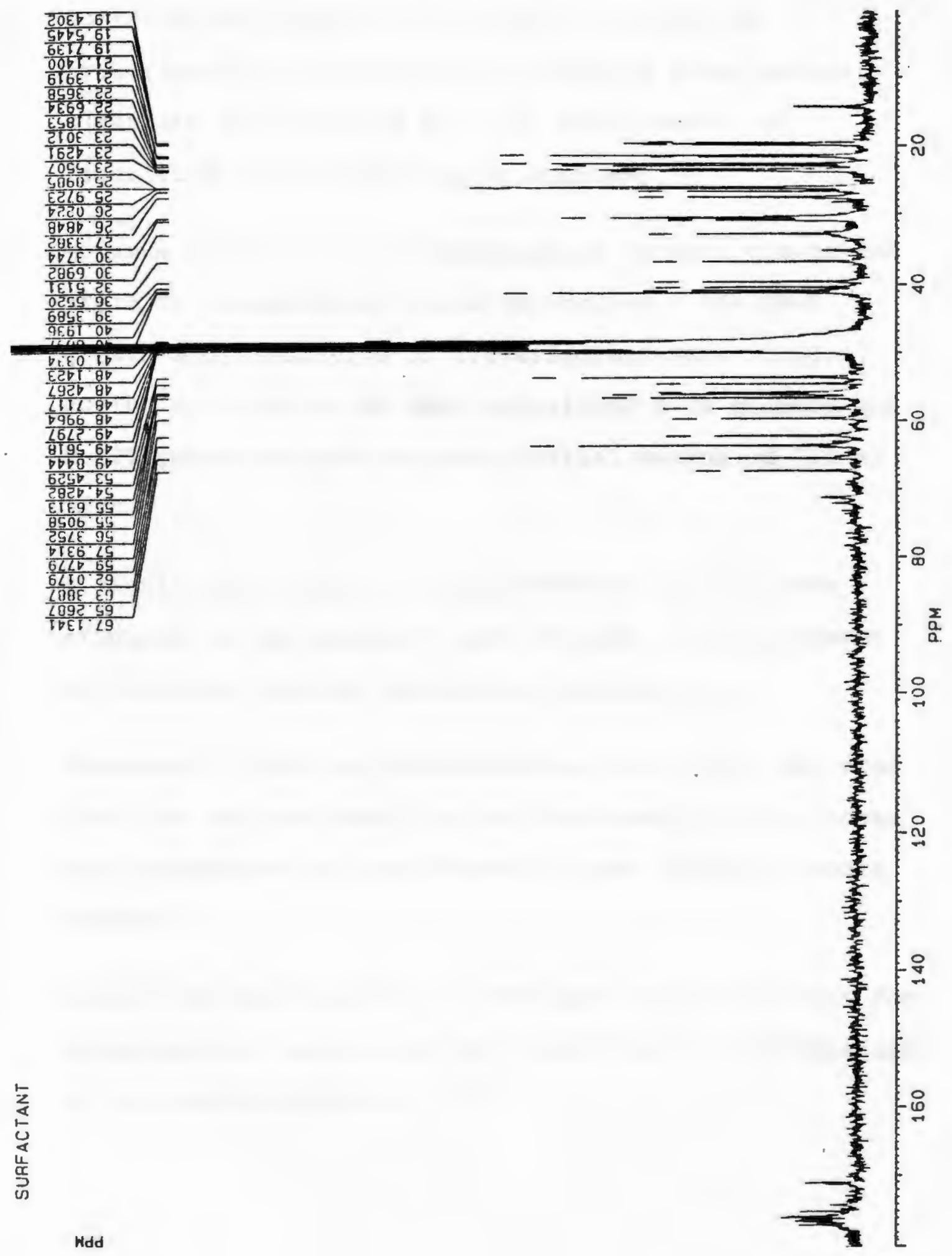




\section{CONCLUSIONS}

1. A microorganism which is capable of producing biosurfactant from cheap and renewable water-soluble substrate was isolated from the environment and identified as an Arthrobacter species.

2. Optimum conditions for Arthrobacter JIZAN-1 growth and yield of biosurfactant were determined. The best growth and production of biosurfactant were obtained during cultivation on MMSM containing $0.2 \%$ glucose and $0.02 \%$ yeast extract and with initial medium $\mathrm{pH} 7.0$ at $30^{\circ} \mathrm{C}$.

3. A significant amount of biosurfactant activity was produced at the cheapest rate through the development of a carbon limited continuous fermentation.

4. Proteins, lipids and carbohydrates which were excreted into the culture broth during the fermentation process were determined by a colorimetric and chromatographic methods.

5. Chloroform was found to be the best organic solvent for biosurfactant extraction after shifting the temperature of the culture broth to $35^{\circ} \mathrm{C}$. 
6. A combination of two steps, silica gel column chromatography and $\mathrm{C}_{18}$ silica (reverse phase) column chromatography were utilized for purification, and the purified surface active compound designated AL-Hazmi's surfactant.

7. AL-Hazmi's surfactant reduced the surface and interfacial tensions of the deionized $\mathrm{H}_{2} \mathrm{O}$ from 73.0 dynes/cm and 18.0 dynes/cm to less than 27.0 dynes $/ \mathrm{cm}$ and 2.0 dynes $/ \mathrm{cm}$.

8. Fatty acids released after acid and alkaline hydrolysis consisted of a number of homologs from $\mathrm{C}_{10}$ to $\mathrm{C}_{20}$ saturated and unsaturated fatty acids, including $\alpha$-branched $\beta$-hydroxy fatty acids which were detected by GC after acid hydrolysis.

9. The water-soluble portion obtained after acid hydrolysis yielded raffinose, galacturonic acid and four unidentified sugars or sugar derivatives.

10. The structure was also examined by means of ' $\mathrm{H}$ and ${ }^{13} \mathrm{C}$ nuclear magnetic resonance (NMR) and by infrared spectrophotometer (IR), and it was found consistent with the normal splitting pattern of similar types of glycolipids. 


\section{LITERATURE CITED}

Abbott, B.J. and W.E. Gledhill, 1971. The extracellular accumulation of metabolic products by hydrocarbon degrading microorganisms. Adv. Appl. Microbiol., $14: 249-388$.

Adams, G.A. 1965. Complete acid hydrolysis. In Methods in Carbohydrate Chemistry. Vol. 5. Academic Press, New York and London/

AL-HAZMI, M.I., K.R. Stauffer, C. Olney, 1985. A new derivative for improved GLC separation of mannose, galactose and glucose, chromatographic Vol. $20(7), 403-406$.

Arima, K., A. Kakinima, and G. Tamura, 1968. Surfactina crystalline peptide-lipid surfactant produced by $B$. subtilis, Biochem, Biophys. Res. Commun., 21(3), 488.

Asselineau, C., H. Montrozier, J.C. Prome, A. Savagnac, and M. Welby, 1972. Etuddun glycolipide polyinsature synthesis par Mycobacterium phlei. Eur. J. Biochem. $28: 102$.

Basta, N., 1983. New biopolymer vies for many surfactant uses, Chem. Eng., 90, May.

Batrakov, S.G., B.V. Rozynov, T.V. Koronelli, and L.D. Bergelson, 1981. Two movel types of Trehalose lipids. Chem. Phys. Lipids. 29:241.

Beebe, J.L. and W.W. Umbriet, 1971. Extracellular lipid of Thiobacillus thiodixans. J. Bacteriol. 108(1), 612 .

Brennan, P.J., D.P. Lehane, and D.W. Thomas, 1970. A cylglucoses of corynbacteria and mycobacteria. Eur. J. Biochem. 13:117-123.

Buchannan, R.E., and N.E. Gibbson. 1974. Bergey's manual of determinative bacteriology, 8th edition. The Williams and Wilkins Company, Baltimore.

Burger, M.M., L. Glaser, and R.M. Rurton, 1963. The enzymatic synthesis of a rhamnose-containing glycolipid by extracts of Pseudomonas aeruginosa.

J. Biol. Chem. 238:2595-2602.

Cirigliano, M.C., and G.M. Carman, 1984. Isolation of a bioemulsifier from Candida lipolytica. Appl. Environ. Microbial. 4 $\overline{8(4)}, 74 \overline{7-750}$. 
Cirigliano, M.C., and G.M. Carman, 1985. Purification and characterization of liposan, a bioemulsifier from Candida lipolytica. Appl. Environ. Microbial. 50(4), 846-850.

Cooper, D.G., B.G. Goldenberg, 1987. Surface active agents from two Bacillus species. Appl. Environ. Microbiol., $53(2), 224-229$.

Cooper, D.G., C.R. MacDonald, S.J.B. Duff, and N-kosaric. 1981. Enhanced production of surfactant from Bacillus subtilis by continuous product removal and metal cation addition, Appl. Environ. Microbiol. 42, 408.

Cooper, D.G., and D.A. Paddock, 1984. Production of a biosurfactant by Torulopsis bombicola. Appl. Environ. Microbiol, 47:173-176.

Cooper, D.G., and D.A. Paddock, 1983. Torulopsis petrophilum and surface activity. Appl. Environ. Microbiol. 46(7), 1426.

Cooper, D.G., J. Akit, and N. Kosaric. 1982. Surface activity of the cells and extracellular lipids of Corynebacterium fascians CF15.J Ferment. Technol. 60:19-24.

Cooper, D.G., J.E. Zajic, 1980. Surface active compounds from microorganisms. Adv. Appl. Microbiol., $26: 229-253$.

Cooper, D.G., J.E. Zajic and D.E. Gerson, 1979. Production of surface-active lipids by Corynebacterium lepus, Appl. Environ. Microbiol., 37, 4 .

Cooper, D.G., J.E. Zajic, D.F. Gerson, and K.I. Manninen, 1980. Isolation and identification of biosurfactants produced during anaerobic growth of Clostridium pasteurinum, J. Ferment. Technol., 58, 83 .

Cutler, A.J., and Light, R.J. 1979. Regulation of Hydoxydocosanoic acid sophoroside production in Candida bogoriensis by the levels of glucose and yeast extract in the growth medium. J. Biological Chemistry, 254(6), 1944-1950.

Deml, G. T. Anke, F. Oberwinkler, B.M. Giannetti, and W. Sleglich, 1980. Schizonellin A and B. New glycolipids from Schizonella Melanogramma, Phytochem., 19, 83.

Donaldson, E.C., and J.B. Clark, eds. 1983. Microbial enhanced oil recovery, Bertlesville Energy Technol Center, 219. 
Dreywood, R. 1946. Quantitative test for carbohydrate material. Ind. Engin. Chem. Anal. Edition, 18:499.

Dubois, M. 1956. Colorimetric method for determination of sugars and related substances. Anal. Chem., $28: 350-356$.

Duvnjak, Z., D.C. Cooper, and N. Kosaric, 1983, in microbial enhanced oil recovery (J.E. Zajic, D.G. Cooper, T.R. Jack, N. Kosavic, eds.) Pennwell Books, Tulsa, Oklahoma.

Duvnjak, Z., D.G. Cooper, and N. Kosaric, 1982. Production of surfactant by Arthrobacter paraffineus ATCC 19558. Biotechnol. Bioeng. XXIV. 165-175.

Duvnjak, Z. and N. Kosaric, 1981. Release of surfactant from Corynebacterium lepus with alkenes. Biotechnology letters, 3(10), 583-588.

Duvnjak, Z. and N. Kosaric, 1985. Production and release of surfactant bo Corynebacterium lepus in hydrocarbon and glucose media. Biotechnology letters, 7(11), 793-796.

Edwards, J. and Hayaski, 1965. Structure of a rhamnolipid from Pseudomonas aeruginosa, Arch. Biochem. Biophys. $11 \overline{1,415 .}$

Fluharty, A.L. and J.S. O'Brien. 1969. Mannose and Erythritol-containing glycolipid from Ustilago maydis. Biochemistry. 8(6):2627.

Frautz, B., S. Lang, and F. Wagner, 1984. Biosurfactant production by Ustilago mydis, in Proc. III. Eur. Cong. Biotechnol, 1-79.

Garrett, H.E. 1972. Surface active chemicals. Pergamon. New York.

Gerson, D.F. and J.E. Zajic, 1978a. Surfactant production from hydrocarbons by Corynebacterium Lepus, sp. and Pseudomonas asphaltenicus, sp. Dev. Ind. Microbiol., $19: 577$.

Gerson, D., and J. Zajic, 1979. The biophysics of cellular adhesion, in Immobilized microbial cells, ACS Symp. No. 106, Washington, D.C., 29.

Goren, M.B., 1970. Sulfolipid I of Mycobacterium Tuberulosis, strain $\mathrm{H}_{37} \mathrm{Rv}$. I. Purification and properties. Biochem. Biophys. Acta. 210:116. 
Goren, M.B., 1970. Sulfolipid I of Mycobacterium Tuberculosis. Strain $\mathrm{H}_{37}$ Rv. II. Structure studies, Biochem. Biophys. Acta. 210, 127.

Gorin, P.A.J., , J.F.T. Spencer, and A.P. Tullock, 1961. Hydroxy fatty acid glycosides of sophorose from Torulopsis magnoliae. Can. J. Chem., 39, 846-855.

Guerra-Santos, L., O. Kappeli and A. Fiechter, 1984. Pseudomonas aeuruginosa biosurfactant production in continuous culture with glucose as carbon source. Appl. Environ. Microbiol., 48:301-305.

Gunstone, F.D. 1967. An introduction to the chemistry and biochemistry of fatty acids and their glycerdies, Champman and Hall, Ltd., Great Britain.

Helenius, A. and K. Simons, 1975. Solubilization of membranes by detergents. Biochem. Biophys. Acta, 415, 29.

Hisatsuka, K., T. Nakahara, N. Sano, and K. Yamada, 1971. Formation of rhamnolipid by Pseudomonas aeruginosa and its function in hydrocarbon fermentation. Agric. Biol., Chem. 35:686-692.

Histasuka, K., T. Nakahara, and K. Yamada, 1972. Protein-like activator for n-alkane oxidation by Pseudomonas aeruginosa $\mathrm{S}_{7} \mathrm{~B}_{1}$. Agric. Biol. Chem. $36: 1361-1369$.

Histasuka, K., T. Nakahara, T. Minoda, and K. Yamada, 1977. Formation of protein-like activator from n-alkane oxidation and its properties. Agric. Biol. Chem., $41: 445-450$.

Holdom, R.S. and A.G. Turner, 1969. Growth of Mycobacterium rhodochrocus on n-decane: a new growth factor and emulsifying agents. J. Appl., Bacteriol., 34:448-456.

Iguchi, I., I. Takeda, and M. Ohsana, 1969. Emulsifying factor of hydrocarbon produced by a hydrocarbon-assimilating yeast. Agric. Biol. Chem. $33(11), 1657$.

Inoue, S., and S. Ito, 1982. Sophorolipids from Torulopsis bombicola as microbial surfactants in alkane fermentations. Biotechnol. Lett. 4:3-8.

Itoh, S., H. Honda, F. Tomita, and T. Suzuki, 1971. Rhamno lipids produced by Pseudomonas aeruginosa grown in n-paraffin, J. Antibiot., 24, 855 . 
Itoh, S. and T. Suzuki, 1974. Fructose-lipids of Arthrobacter, Corynebacteria, Nocardia, and Mycobacteria grown on fructose. Agric. Biol. Chem. 38:1443-1449.

Javaheri, M., G.E. Jenneman, M.J. McInerney, and R.M. Knapp, 1985. Anaerobic production of a Biosurfactant by Bacillus Licheniformis JF-2. Appl. Environ. Microbial. 50(3) 698-700.

Jenneman, G.E., M.J. McInerney, R.M. Knapp, J.B. Clark, J.M. Feero, D.E. Revus, and D.E. Menzie, 1983.

Ahalotolerant, biosurfactant-producing Bacillus species potentially useful for enhanced oil recovery. Dev. Ind. Microbiol. 24:485-492.

Jobson, A., F.D. Cook and D.W. S. Westlake, 1972. Microbial utilization of crude oil. Appl. Microbiol., $23: 1082-1089$.

Kaepeli, O., and A. Fiechter, 1977. Component from the cell surface of the hydrocarbon-utilizing yeast Candida tropicalis with possible relation to hydrocarbon transport. J. Bacteriol. 131:917-921.

Kaeppeli, O. and A. Fiechter, 1976. The mode of interaction between the substrate and cell surface of the hydrocarbon utilizing yeast Candida tropicalis. Biotechnol. Bioeng. 18, 967.

Kaeppeli, O. and W.R. Finnerly. 1980. Characteristics of hexadecane partition by the growth medium of Acinetobacter sp. Biotechnol. Bioeng. 22:495-503.

Kakinuma, A., M. Hori, M. Isono, G. Tamura, and K. Arima, 1969. Determination of amino acid sequence in surfactin, a crystalline peptide lipid surfactant produced by Bacillus subtilis, Agric. Biol. Chem., $33(6), 971$.

Kakinuma, A., H. Sugino, M. Isono, G. Tamura, and K. Arima, 1969. Determination of fatty acid in surfactin and elucidation of the total structure of surfactin, Agric. Biol. Chem., 33(6), 973.

Kakinuma, A., M. Hori, H. Sugino, I. Toshida, M. Isono, G. Tamura, and K. Arima, 1969. Determination of the location of lactonse ring in surfactin, Agric. Biol., Chem. , 33, 1523.

Kates, M. 1972. techniques of lipidology: Isolation analysis and Identification of lipids. North-Holland, New York, pp. 347-353. 
Kawanami, J., A. Kimura and H. Oksuka, 1968. Siolipin A: A new lipoamino acid ester isolated from Streptomyces sioyaensis. Biochem. Biophys. Acta. 152:808.

Kawashima, H., T. Nakahara, M. Oogaki and T. Tabuchi, 1983. Extraceliular production of a Mannosyl erythritol lipid by a mutant of Candida sp. from $n-a l k a n e s$ and triglycerols. J. Ferment. Technol., 61(2):143.

Knoche, H. and J. Shireley, 1972. Structure of an ornithine-containing lipid from Thiobacillus thioxidans, J. Biol., Chem., 247, 170.

Kosaric, N., N.C.C. Gray and W.L. Cairns, 1983. Microbioal emulsifiers and de-emulsifiers in Biotechnology, H.T. Rehm and G. Reed, eds. Verlag Chemic Weinheim, Vol. 3, pp. 575-592.

Kosaric, N., W.L. Cairns and N.C.C. Gray. 1987. Biosurfactants and biotechnology, Vol. 25, Marcel Dekker, New York.

Krelschmer, A., H. Bock and F. Wagner, 1982. Chemical and physical characterization of interfacial-active lipids from Rhodococcus erythropolis grown on n-alkanes. Appl. Environ. Microbiol. 44:864-870.

Lang, S., A. Gilbon, C. Syldatk and F. Wagner, 1984. Surfactant in solution. Vol. 2 (K.L. Mittal and B. Lindmann, eds.), Plenum, New York, 1365-1376.

Li, Z.Y., S. Lang, F. Wagner, L. Witte, and V. Wray, 1984. Formation and identification of interfacial-active glycolipid from resting microbial cells. Appl. Environ. Microbiol. 48:610-617.

Lowry, O. H., N.J. Rosebrough, A.L. Farr and R.J. Randall, 1951. Protein measurement with the folin phenol reagent. J. Biol. Chem., 193:265-275.

MacDonald, C.R., D.G. Cooper, and J.E. Zajic, 1981. Surface-active lipids from Nocardia erythropolis grown on hexadecane, Appl. Environ. Microbiol., 41, 117.

Makula, R.A., and W.R. Finnerty, 1972. Microbiol assimilation of hydrocarbons: cellular distribution of fatty acids. J. Bacteriol. 112, 398-407.

Makula, R.A., P.J. Lockwood, and W.R. Finnerty, 1975. Comparative analysis of the lipids of Acientobacter species grown in hexadecane. J. Bacteriol. 121, 250-258. 
Margaritis, A., J.E. Jajic and D.F. Gerson, 1979. The production of surface active properties of microbial surfactants. Biotechnol. Bioeng. 21,1151-1162.

Margaretis, A., K. Kennedy, J. Zajic and Gerson, 1979. Biosurfactant production by Nocardia erythropolis. Dev. Ind. Microbiol., 20:623.

Matales, R.I., J.N. Baruah and S.R. Tannenbaum, 1968. Growth of a thermophilic bacterium on hydrocarbon: a new source of single cell protein. Science, $157: 1322-1323$.

Matsuyama, T., T. Murakami, M. Fujita, S. Fujita, and I. Yano, 1986. Extracellular vesicle formation and biosurfactant production by serratia marcescens. Microbiol. 132, 865-875.

Mulligan, C.N., D.G. Cooper, and R.J. Newfeld, 1984. Selęction of microbes producing biosurfactants in media without hydrocarbons. J. Ferment. Technol., 62, 311.

Nakao, Y., T. Kanamura, M. Kikuchi, and S. Yamatodani, 1973. Action of pencillin on membrane permeability barrier to L-glutamic and Agric. Biol., Chem., 37, 2399.

Neufeld, R., J. Zajic, and D. Gerson, 1980. Cell surface measurements in hydrocarbon and carbohydrate fermentations. Appl. Environ.

Panchal, C.J. and J.E. Zajic, 1978. Isolation of emulsifying agents from a species of Corynebacterium. Dev. Ind. Microbiol., 19, 569.

Pomeranz, Y., and C.E. Meloan, 1978. Food analysis: Theory and Practice, AVI Publishing Company, Inc. Westport, Connecticut.

Rapp, P. and F. Wagner, 1976. Formation of trehalose lipid by Nocardia species grown on n-alkanes. Presented at the fifth international fermentation symposium.

Rapp, R., H. Bock, V. Wray, and F. Wagner, 1979. Formation, isolation and characterization of trehalose dimycolates from Rhodococcus erythropolis grown on n-alkalanes. J. Gen. Microbiol., 115:491-503.

Reddy, R.G., H.D. Singh, M.G. Pathals, S.D. Bhagat, and J.N. Baruah. 1983. Isolation and functional characterization of hydrocarbon emulsifying and solubilizing factors produced by a Pseudomonas species. Bootechnol. Bioeng. 25:387-401. 
Reiling, H.E., U. Thanei-Wyss, L.H. Guerra-Santos, R. Hirt, O. Kappeli and A. Fiechter, 1986. Pilot plant production of rhamnolipid biosurfactant by Pseudomonas aeruginosa, Appl. Environ. Microbiol. 51(5), 985-989.

Ristau, R. and F. Wagner, 1983. Formation of novel anionic trehalosetetraesters from Rhodococcus erythropolis under growth limiting conditions. Biotechnol, Lett. 5:95-100.

Rosenberg, E., A. Perry, D.T. Gibson and D.L. Gutnick, 1979b. Emulsifier of Arthrobacter RAG-1: specificity of hydrocarbon substrate. Appl. Environ. Microbiol. 37, 409-413.

Rosenberg, E., A. Zuckerberg, C. Rubinowitz, and D.L. Gutnick, 1979a. Emulsifier of Arthrobacter RAG-1: isolation and emulsifying properties, Appl. Environ. Microbiol., 37, 402-408.

Rosenberg', E., C. Rubinovitz, A. Gottlieb, S. Rosenhak, and E.Z. Ron, 1988. Production of biodispersan by Acinetobacter calcoaceticus AZ-Appl. Environ. Microbiol. 54 $\left(\frac{2}{2)}, 317-322\right.$.

Rosenberg, E., C. Rubinovitz, R. Legmann and E.z. Ron, 1988. Purification and chemical properties of Acinetobacter calcoaceticus AZ Biodisperson. Appl. Environ. Microbiol 54(2), 323.

Shaw, N., 1970. Bacterial glycolipids, Bacteriol Rev., 34,365 .

Shaw, N., 1974. Lipid composition as a guide to the classification of bacteria, Adv. Appl. Microbiol., 17,63 .

Shoham, Y. and E. Rosenberg. 1983. Enzymatic depolymerization of emulsan. J. Bacteriol. 156:161-167.

Singer, M.E., W.R. Finnerty, P. Bolden, and A.D. King, 1983. Characterization of a biosurfactant effective in heary oil viscosity reduction, Am. Chem., Soc. Div. Petrol. Chem., 785.

Sittig, M. and R. Noyes, 1985. Genetic engineering and Biotechnology firms worldwide Directory 4 th ed. Sittig and Noyes, New Jersey, 501.

Spencer, J., D. Spencer, and A. Tulloch, 1979. Extracellular glycolipids of yeasts, in Economic Microbiology, Vol. 3, Rose, A., Ed., Academic Press, London, 523 . 
Suzuki, T., H. Tanaka, and S. Itah, 1974. Sucrose lipids of Arthrobacteria, Corynebacteria and Nocardia grown on sucrose. Agric. Biol. 38:557-563

Suzuki, T., and K. Ogawa. 1972. Transcient accumulation of fatty alcohols by n-paraffin grown microorganisms. Agric. Biol. Chem., 36:457-463.

Suzuki, T., K. Tanaka, I. Natoubara and S. Kinoshita. 1969. Trehalose lipid and $\alpha$-branched- $\beta$-hydroxy fatty acid formed by bacteria grown on n-alkanes, Agric. Biol. Chem., 33(11), 1619.

Suzuki, T., K. Tanaka, and S. Kinoshita. 1969. The extracellular accumulation of trechalose and glucose by bacteria grown on n-alkanes. Agric. Biol. Chem., $33(2), 190$.

Sweeley, C.C., R. Bentley, M. Makita and W.W. Wells, 1963. Gas liquid chromatography trimethylsilyl derivatives of sugars and related substances. J. Am. Chem. SOC., $85: 2497-2507$.

Swisher, R.D. 1970. Surfactant Biodegradation, Marcel Dekker, New York.

Tahara, Y., M. Kameda, Y. Yamada, and K. Kondo, 1976. A new lipid, the ornithine and taurine-containing "Cerilipin", Agric. Biol., Chem., 40, 243.

Tahara, Y., T. Tamada, and K. Konda, 1976. A new lysinecontaining lipids isolated by Agrobacterium tumefaciens. Agric. Biol. Chem., 40, 1449 .

Traxler, R.W., and J.M. Bernard. 1968. The utilization of $\mathrm{n}$-alkanes, by Pseudomonas aeruginosa under conditions of anaerobiosis. I. Preliminary observation. Intl. Biodetn. Bull. 5:21-25.

Tullock, A.P.J., F.F. Spencer, and P.A.J. Gorin. 1962. The fermentation of long-chain compounds by Torulopsis magnoliae. Can. J. Chm. 40:1326-1339.

Wagner, F., H. Bock, and A. Kretschmer, 1980. in Fermentation. II. Rotenburger symposium (R.M. Lafferty, ed.), 181-192.

Wagner, F., J.S. Lang, Z.Y. Li, G. Marweda, U. Matulovic, E. Ristau and C. Syldatk, 1984. Production of surface active anionic glycolipids by resting and immunobilized microbial cells. III. Eur. Cong. Biotechnol. 1-3. 
Wagner, F., U. Behrendt, H. Bock, A. Kretschmer, S. Lang, and C. Syldatk 1983, in Microbial enhanced oil recovery (J.E. Zajic, D.G. Cooper, T.R. Jack, N. Kosaric, eds.), Pennwell Books, Tulsa, Oklahoma.

Wicken, A.J. and K.W. Knox, 1970. Studies on the group F antigen of Lactobacilli: Isolation of a Teichnoic acid-lipid complex from Lactobacillus fermenti NCTC 6991. J. Ge. Microbiol., 60, 293.

Wilkinson, S., 1972. Composition and structure of the ornithine-containing lipid from pseudomonas rubescens, Biochem, Biophys. Acta, 270, 1 .

Yamaquchi, M., A. Sato, and A. Uakuyama. 1976. Microbial production of sugar-lipids. Chem. Ind. (London) $4: 741-742$.

Zajic, J.E. and B. Supplisson, 1972. Emulsification and degnadation of "Bunker C" fuel oil microorganisms. Biotechnol. Bioeng. XIV, 331.

Zajic, J.E., B. Supplisson and B. Volesky, 1974. Bacterial degradation and emulsification of No. 6 fuel oil, Environ. Sci. Technol. 8, 664 .

Zajic, J.E., D.F. Gerson and S.E. Comp. 1977a. Biodegradation of asphaltenes and other hydrocarbons by Pseudomonas sp. Can. Fed. Biol., Soc. 20:33.

Zajic, J.E., H. Guignard, and D.F. Gerson, 1977b. Emulsifying and surface active agents from Corynobacterium hydrocarboblastus. Biotechnol. Bioeng. 19:1285-1301.

Zajic, J., H. Guignard and D. Gerson, 1977. Properties and biodegradation of a bioemulsifier from Corynebacterium hydrocarboclastus. Biotechnol. Bioeng. 19, 1303 .

Zuckerberg, A., A Diver, Z. Peeri, D.L. Gutnick and E. rosenberg, 1979. Emulsifier of Arthrobacter RAG-1: chemical and physical properties. Appl. Environ. Microbial., 37, 414-420. 
-259- 